Supporting Information

\title{
A C-H Insertion Approach to Functionalized Cyclopentenones
}

\footnotetext{
Youliang Wang, ${ }^{\dagger}$ Maxence Zarca, ${ }^{\dagger}$ Liu-Zhu Gong, ${ }^{\ddagger}$ and Liming Zhang ${ }^{\dagger *}$

† Department of Chemistry \& Biochemistry, University of California, Santa Barbara, CA 93106

‡ Hefei National Laboratory for Physical Sciences at Microscale and Department of Chemistry, University of Science and Technology of China, Hefei, 230026 (China)
}

\begin{tabular}{|l|l|}
\hline Contents & \multicolumn{1}{|l|}{$\begin{array}{l}\text { Page } \\
\text { number }\end{array}$} \\
\hline General & $\mathrm{S} 2$ \\
\hline General procedure A/B/: preparation of substrates & $\mathrm{S} 2$ \\
\hline $\begin{array}{l}\text { General procedure C/D/E: Gold-catalyzed cyclopentenones synthesis } \\
\text { via intramolecular insertions into unactivated C(sp })^{\mathbf{3}} \text {-H bonds }\end{array}$ & $\mathrm{S} 13$ \\
\hline${ }^{\mathbf{1}} \mathbf{H}$ and ${ }^{\mathbf{1 3}} \mathbf{C}$ NMR spectra & $\mathrm{S} 24$ \\
\hline
\end{tabular}


General. Ethyl acetate (ACS grade), hexanes (ACS grade), diethyl ether (ACS grade), were purchased from Fisher Scientific and used without further purification. Anhydrous toluene and 1,2-dichloroethane were bought from Acros and used directly. Commercially available reagents were used without further purification. Reactions were monitored by thin layer chromatography (TLC) using Silicycle precoated silica gel plates. Flash column chromatography was performed over Silicycle silica gel (230-400 mesh). ${ }^{1} \mathrm{H}$ NMR and ${ }^{13} \mathrm{C}$ NMR spectra were recorded on a Varian $400 \mathrm{MHz}$ spectrometer, a Varian $500 \mathrm{MHz}$ Unity plus spectrometer, and a Varian $600 \mathrm{MHz}$ Unity plus spectrometer, using residue solvent peaks as internal standards $\left(\mathrm{CDCl}_{3},{ }^{1} \mathrm{H}: 7.26 \mathrm{ppm} ;{ }^{13} \mathrm{C}: 77.00 \mathrm{ppm}\right)$. Infrared spectra were recorded with a Perkin Elmer FT-IR spectrum 2000 spectrometer and are reported in reciprocal centimeter $\left(\mathrm{cm}^{-1}\right)$. Mass spectra were recorded with Waters micromass ZQ detector using electrospray method (MeCN as solvent).

\section{General Procedure A: preparation of ynone 1a-TMS}

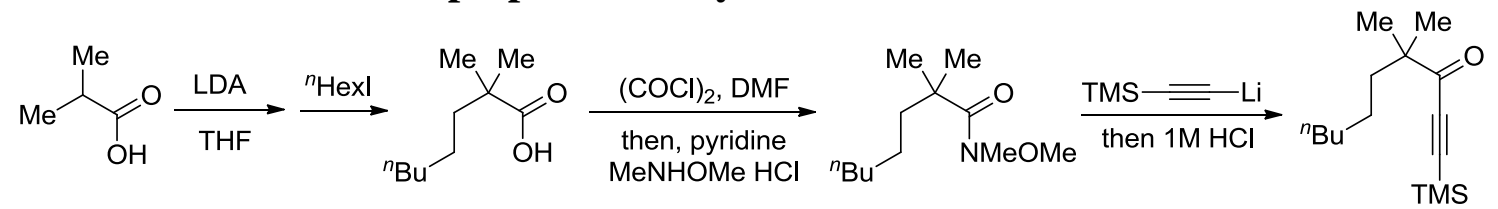

LDA (110 mmol) was added dropwise to a mixture of isobutyric acid $(4.05 \mathrm{~mL}, 44 \mathrm{mmol})$ and THF $(100 \mathrm{~mL})$ at $-78^{\circ} \mathrm{C}$, and the mixture was allowed to warm up to room temperature and stirred for $1 \mathrm{~h}$. Then the reaction mixture was recooled to $-78^{\circ} \mathrm{C}$ and hexyl iodide $(6.5$ $\mathrm{mL}, 44 \mathrm{mmol}$ ) was added dropwise. The resulting solution was allowed to warm up to room temperature and stirred overnight. After the reaction finished, the reaction mixture was quenched with $10 \% \mathrm{HCl}$ solution and extracted with DCM. The combined organic extract was washed with brine, dried over anhydrous $\mathrm{MgSO}_{4}$, and concentrated under reduced pressure to give 2,2-dimethyloctanoic acid (6.2 g, crude), which, without purification, was dissolved in DCM $(60 \mathrm{~mL})$. One drop of DMF was added to the above solution, followed by the dropwise addition of $(\mathrm{COCl})_{2}(3 \mathrm{~mL})$. The resulting mixture was stirred for $1 \mathrm{~h}$ at room temperature and then concentrated under reduced pressure to give 2,2-dimethyloctanoyl chloride, which, without purification, was dissolved in DCM (100 $\mathrm{mL})$. To the above mixture was added $N, O$-dimethylhydroxylamine hydrochloride $(3.3 \mathrm{~g})$, pyridine $(7.3 \mathrm{~mL})$, and catalytic amout of DMAP at $0^{\circ} \mathrm{C}$. The resulting solution was allowed to warm up to room temperature and stirred overnight. After the reaction finished, the reaction mixture was quenched with $10 \% \mathrm{HCl}$ solution and extracted with DCM. The combined organic extract was washed with brine, dried over anhydrous $\mathrm{MgSO}_{4}$, and concentrated under reduced pressure to give N-methoxy-N,2,2-trimethyloctanamide (6.3 $\mathrm{g}$, crude). $1.29 \mathrm{~g}(6 \mathrm{mmol})$ of the above crude product was dissolved in THF $(10 \mathrm{~mL})$. To the above solution was added a THF $(20 \mathrm{~mL})$ solution of ((trimethylsilyl)ethynyl)lithium $(9 \mathrm{mmol})$ at $-78^{\circ} \mathrm{C}$. The resulting solution was allowed to warm up to room temperature within 2 hours. After the reaction finished, the reaction mixture was quenched with $1 \mathrm{M}$ $\mathrm{HCl}$ solution $(12 \mathrm{~mL})$ and extracted with $\mathrm{Et}_{2} \mathrm{O}$. The combined organic extract was washed with brine, dried over anhydrous $\mathrm{MgSO}_{4}$, and concentrated under reduced pressure. The residue was purified by chromatography on silica gel to afford the desired product 1a-TMS (1.36 g, $5.4 \mathrm{mmol}, 60 \%$ yield from isobutyric acid in three steps).

\section{General Procedure B: preparation of ynone 1i}




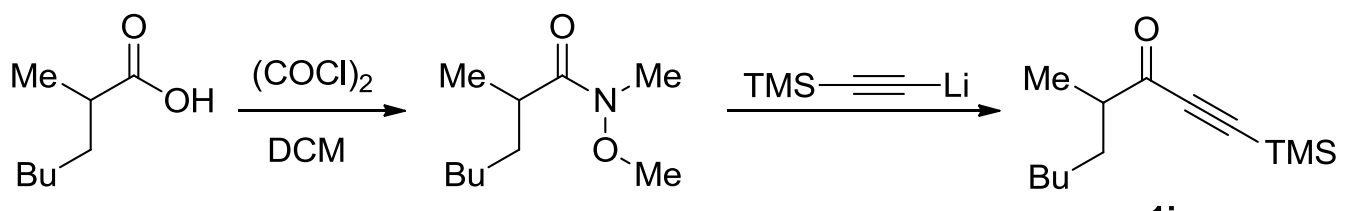

$1 \mathbf{i}$

2-Methylheptanoic acid (1.44 g, $10 \mathrm{mmol}$ ) was dissolved in DCM (30 mL). One drop of DMF was added to the above solution, followed by the dropwise addition of $(\mathrm{COCl})_{2}(1.3$ $\mathrm{mL})$. The resulting mixture was stirred for $1 \mathrm{~h}$ at room temperature and then concentrated under reduced pressure to give the corresponding acid chloride, which, without purification, was dissolved in DCM $(30 \mathrm{~mL})$. To the above mixture was added $\mathrm{N}, \mathrm{O}$ dimethylhydroxylamine hydrochloride $(1.05 \mathrm{~g})$, pyridine $(2.4 \mathrm{~mL})$, and catalytic amout of DMAP at $0^{\circ} \mathrm{C}$. The resulting solution was allowed to warm up to room temperature and stirred overnight. After the reaction finished, the reaction mixture was quenched with $10 \%$ $\mathrm{HCl}$ solution and extracted with DCM. The combined organic extract was washed with brine, dried over anhydrous $\mathrm{MgSO}_{4}$, and concentrated under reduced pressure to give the corresponding weinreb amide. $374 \mathrm{mg}(2 \mathrm{mmol})$ of the above crude product was dissolved in THF (4 mL). To the above solution was added dropwise ((trimethylsilyl)ethynyl)lithium ( $3 \mathrm{mmol}$, in $5 \mathrm{~mL} \mathrm{THF}$ ) at $-78^{\circ} \mathrm{C}$. The resulting solution was allowed to warm up to room temperature within 2 hours. After the reaction finished, the reaction mixture was quenched with $1 \mathrm{M} \mathrm{HCl}$ solution $(12 \mathrm{~mL})$ and extracted with $\mathrm{Et}_{2} \mathrm{O}$. The combined organic extract was washed with brine, dried over anhydrous $\mathrm{MgSO}_{4}$, and concentrated under reduced pressure. The residue was purified by chromatography on silica gel to afford the desired product $\mathbf{1 i}$.

\section{4,4-dimethyl-1-(trimethylsilyl)dec-1-yn-3-one (1a-TMS)<smiles>C#CC#CC(=O)C(C)(C)CCBr</smiles>

1a-TMS

Compound 1a-TMS was prepared following the general procedure A. ${ }^{1} \mathrm{H}$ NMR $(500 \mathrm{MHz}$, Chloroform- $d$ ) $\delta 1.61-1.56(\mathrm{~m}, 2 \mathrm{H}), 1.33-1.15(\mathrm{~m}, 8 \mathrm{H}), 1.14(\mathrm{~s}, 6 \mathrm{H}), 0.90-0.84(\mathrm{~m}$, $3 \mathrm{H}), 0.24(\mathrm{~s}, 9 \mathrm{H}) ;{ }^{13} \mathrm{C} \mathrm{NMR}\left(126 \mathrm{MHz}, \mathrm{CDCl}_{3}\right) \delta$ 194.07, 100.46, 98.82, 48.00, 39.74, 31.56, 29.81, 24.49, 23.79, 22.55, 14.02, -0.74; IR (neat): 2962, 2932, 2859, 2149, 1673, 1470, 1253, 1096, 846; $\mathrm{ESI}^{+}$calculated for $\left[\mathrm{C}_{15} \mathrm{H}_{28} \mathrm{NaOSi}^{+}:\right.$: 275.18, found 275.11 .

\section{4,4-dimethyldec-1-yn-3-one (1a-H)}

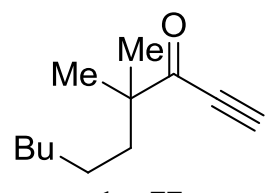

1a-H

Compound 1a-H was prepared following the general procedure $\mathbf{A}$ by replacing the (trimethylsiyl)ethynyl lithium (1.5 equiv) with ethynyl magnisum bromide (2 equiv). ${ }^{1} \mathrm{H}$ NMR (500 MHz, Chloroform- $d$ ) $\delta 3.20(\mathrm{~s}, 1 \mathrm{H}), 1.65-1.56(\mathrm{~m}, 2 \mathrm{H}), 1.31-1.21(\mathrm{~m}, 6 \mathrm{H})$, $1.16(\mathrm{~m}, 8 \mathrm{H}), 0.87(\mathrm{t}, J=6.8 \mathrm{~Hz}, 3 \mathrm{H}) ;{ }^{13} \mathrm{C} \mathrm{NMR}\left(126 \mathrm{MHz}, \mathrm{CDCl}_{3}\right) \delta 193.53,79.86,79.37$, 48.14, 39.44, 31.57, 29.79, 24.43, 23.50, 22.54, 14.01; IR (neat): 2961, 2932, 2089, 1676, 1466, 1387; $\mathrm{ESI}^{+}$calculated for $\left[\mathrm{C}_{12} \mathrm{H}_{20} \mathrm{NaO}^{+}:\right.$203.14, found 203.09. 


\section{4,4-dimethyl-1-(triethylsilyl)dec-1-yn-3-one (1a-TES)}

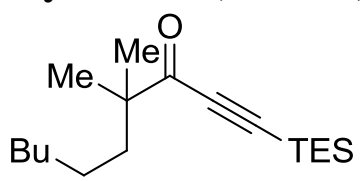

1a-TES

Compound 1a-TES was prepared following the general procedure A by replacing the (trimethylsiyl)ethynyl lithium (1.5 equiv) with (triethylsiyl)ethynyl lithium (1.5 equiv). ${ }^{1} \mathrm{H}$ NMR (500 MHz, Chloroform- $d$ ) $\delta 1.64-1.58(\mathrm{~m}, 2 \mathrm{H}), 1.32-1.17(\mathrm{~m}, 8 \mathrm{H}), 1.15(\mathrm{~s}, 6 \mathrm{H})$, $1.02(\mathrm{t}, J=7.9 \mathrm{~Hz}, 9 \mathrm{H}), 0.90-0.84(\mathrm{~m}, 3 \mathrm{H}), 0.68(\mathrm{q}, J=7.9 \mathrm{~Hz}, 6 \mathrm{H}) ;{ }^{13} \mathrm{C} \mathrm{NMR}(126 \mathrm{MHz}$, $\left.\mathrm{CDCl}_{3}\right) \delta 193.97,101.76,96.88,48.03,39.93,31.61,29.86,24.55,23.81,22.57,14.01$, 7.33, 3.92; IR (neat): 2959, 2933, 2876, 2146, 1673, 1469, 1096, 1067, 729; ESI ${ }^{+}$calculated for $\left[\mathrm{C}_{18} \mathrm{H}_{34} \mathrm{NaOSi}\right]^{+}: 317.23$, found 317.16 .

\section{1-bromo-4,4-dimethyldec-1-yn-3-one (1a-Br)}<smiles>C#CC(=O)C(C)(C)CCBr</smiles>

1a-H

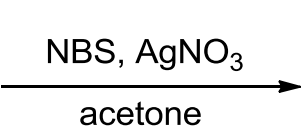

acetone

NBS $(213 \mathrm{mg})$ was added in one portion to a mixture of $\mathbf{1 a - H}(1 \mathrm{mmol}, 180 \mathrm{mg}), \mathrm{AgNO}_{3}$ $(8.5 \mathrm{mg})$, and acetone $(10 \mathrm{~mL})$. The above solution was stirred for 2 hours at room temperature and then concentrated under reduced pressure. The residue was purified by chromatography on silica gel to afford the desired product 1a-Br $(233 \mathrm{mg}, 90 \%) .{ }^{1} \mathrm{H}$ NMR (400 MHz, Chloroform- $d$ ) $\delta 1.62-1.53(\mathrm{~m}, 2 \mathrm{H}), 1.34-1.15(\mathrm{~m}, 8 \mathrm{H}), 1.15(\mathrm{~s}, 6 \mathrm{H}), 0.92-$ $0.83(\mathrm{t}, J=6.7 \mathrm{~Hz}, 3 \mathrm{H}) ;{ }^{13} \mathrm{C} \mathrm{NMR}\left(101 \mathrm{MHz}, \mathrm{cdcl}_{3}\right) \delta 192.58,78.46,57.16,48.31,39.45$, 31.58, 29.75, 24.44, 23.59, 22.56, 14.06; IR (neat): 2960, 2931, 2858, 2174, 1674, 1387, 1103, 1070; $\mathrm{ESI}^{+}$calculated for $\left[\mathrm{C}_{12} \mathrm{H}_{19} \mathrm{NaOBr}\right]^{+}: 281.05,283.05$, found 280.99, 282.99 .

\section{4,4,6-trimethyl-1-(trimethylsilyl)hept-1-yn-3-one (1b)}

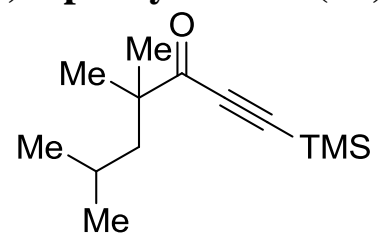

$1 \mathrm{~b}$

Compound 1b was prepared following the general procedure $\mathbf{A}$ by replacing hexyl iodide with 1-bromo-2-methylpropane. ${ }^{1} \mathrm{H}$ NMR $(500 \mathrm{MHz}$, Chloroform- $d$ ) $\delta 1.62(\mathrm{~m}, 3 \mathrm{H}), 1.16$ $(\mathrm{s}, 6 \mathrm{H}), 0.87(\mathrm{~d}, J=6.6 \mathrm{~Hz}, 6 \mathrm{H}), 0.24(\mathrm{~s}, 9 \mathrm{H}) ;{ }^{13} \mathrm{C} \mathrm{NMR}\left(126 \mathrm{MHz}, \mathrm{CDCl}_{3}\right) \delta 194.41$, 100.69, 99.28, 48.71, 48.17, 25.11, 24.60, 24.11, -0.75; IR (neat): 2961, 2931, 2148, 1672, $1471,1456,1252,1060 ; \mathrm{ESI}^{+}$calculated for $\left[\mathrm{C}_{13} \mathrm{H}_{24} \mathrm{NaOSi}\right]^{+}: 247.15$, found 247.09.

\section{4,4-dimethyl-5-phenyl-1-(trimethylsilyl)pent-1-yn-3-one (1c)}




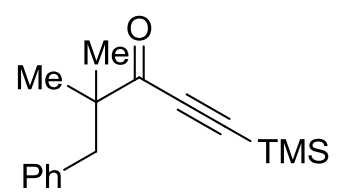

1c

Compound 1c was prepared following the general procedure A by replacing hexyl iodide with benzyl chloride. ${ }^{1} \mathrm{H}$ NMR (500 MHz, Chloroform- $d$ ) $\delta 7.27-7.24(\mathrm{~m}, 2 \mathrm{H}), 7.23-$ $7.19(\mathrm{~m}, 1 \mathrm{H}), 7.14(\mathrm{dd}, J=6.9,1.8 \mathrm{~Hz}, 2 \mathrm{H}), 2.92(\mathrm{~s}, 2 \mathrm{H}), 1.16(\mathrm{~s}, 6 \mathrm{H}), 0.26(\mathrm{~s}, 9 \mathrm{H}) ;{ }^{13} \mathrm{C}$ NMR $\left(126 \mathrm{MHz}, \mathrm{CDCl}_{3}\right) \delta 193.25,137.26,130.27,128.01,126.47,100.50,100.19,49.09$, 45.05, 23.72, -0.73; IR (neat): 3091, 3063, 2967, 2153, 2087, 1671, 1252, 1080, 846; ESI ${ }^{+}$ calculated for $\left[\mathrm{C}_{16} \mathrm{H}_{22} \mathrm{NaOSi}\right]^{+}: 281.13$, found 281.05 .

\section{4,4,5-trimethyl-1-(trimethylsilyl)hex-1-yn-3-one (1d)}

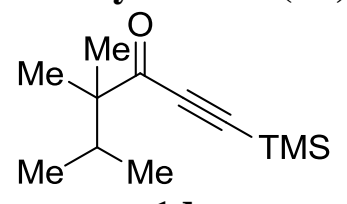

1d

Compound 1d was prepared following the general procedure $\mathbf{A}$ by replacing hexyl iodide with 2-iodo propane. ${ }^{1} \mathrm{H}$ NMR $(500 \mathrm{MHz}$, Chloroform- $d$ ) $\delta 2.20-2.08(\mathrm{~m}, 1 \mathrm{H}), 1.07$ (s, $6 \mathrm{H}), 0.85(\mathrm{~d}, J=6.9 \mathrm{~Hz}, 6 \mathrm{H}), 0.24(\mathrm{~s}, 9 \mathrm{H}) ;{ }^{13} \mathrm{C} \mathrm{NMR}\left(126 \mathrm{MHz}, \mathrm{CDCl}_{3}\right) \delta 194.49,100.70$, 98.79, 51.08, 33.73, 20.05, 17.46, -0.71, -0.72; IR (neat): 2966, 2151, 1672, 1466, 1395, $1252,1083,1049,846 ; \mathrm{ESI}^{+}$calculated for $\left[\mathrm{C}_{12} \mathrm{H}_{22} \mathrm{NaOSi}^{+}: 233.13\right.$, found 233.08.

(1,3-bis(2,6-diisopropylphenyl)-1H-imidazol-2(3H)-ylidene)(3-oxododec-1-yn-1yl)gold (1e-Au)

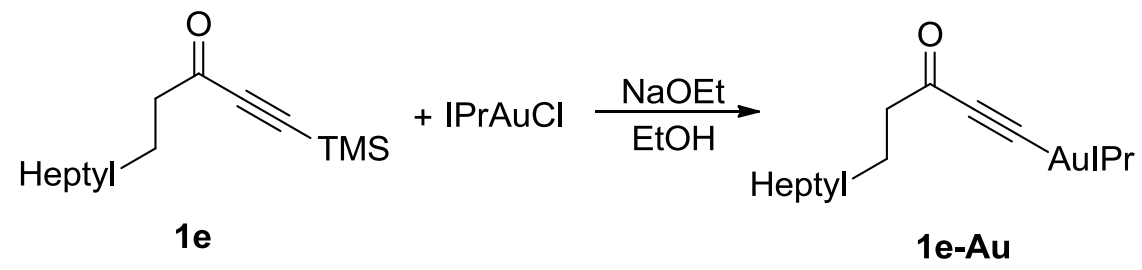

$\mathrm{NaOEt}(0.1 \mathrm{~mL}, 0.5 \mathrm{M})$ was added dropwise to a mixture of $1 \mathbf{e}(13.3 \mathrm{mg}, 0.053 \mathrm{mmol})$, $\mathrm{IPrAuCl}(31 \mathrm{mg}, 0.05 \mathrm{mmol})$, and $\mathrm{EtOH}(1 \mathrm{~mL})$ at room temperature under argon. The mixture was allowed to stir overnight at the same temperature. Then the mixture was quenched with saturated ammonium chloride solution and extracted with ether. The combined organic extract was washed with brine, dried over anhydrous $\mathrm{MgSO}_{4}$, and concentrated under reduced pressure. The residue was purified by chromatography on silica gel to afford the desired product 1e-Au $(26.8 \mathrm{mg}, 70 \%) .{ }^{1} \mathrm{H}$ NMR $(500 \mathrm{MHz}$, Chloroform- $d$ ) $\delta 7.50(\mathrm{t}, J=7.8 \mathrm{~Hz}, 2 \mathrm{H}), 7.29(\mathrm{~d}, J=7.8 \mathrm{~Hz}, 4 \mathrm{H}), 7.14$ (s, 2H), 2.55 (hept, $J=7.0 \mathrm{~Hz}, 4 \mathrm{H}), 2.37(\mathrm{t}, J=7.5 \mathrm{~Hz}, 2 \mathrm{H}), 1.50(\mathrm{~m}, 2 \mathrm{H}), 1.33(\mathrm{~d}, J=6.9 \mathrm{~Hz}, 12 \mathrm{H}), 1.21(\mathrm{~m}$, $24 \mathrm{H}), 0.86(\mathrm{t}, J=7.1 \mathrm{~Hz}, 3 \mathrm{H}) ;{ }^{13} \mathrm{C}$ NMR $\left(126 \mathrm{MHz}, \mathrm{CDCl}_{3}\right) \delta 189.95,188.39,145.56$, $138.20,133.96,130.61,124.18,123.30,103.35,45.74,31.85,29.39,29.36,29.25,29.05$, 28.80, 24.56, 24.30, 23.98, 22.65, 14.09; IR (neat): 3162, 3121, 3076, 2962, 2926, 2868, 2098, 1646, 1491, 803, 758; $\mathrm{ESI}^{+}$calculated for $\left[\mathrm{C}_{39} \mathrm{H}_{55} \mathrm{NaOAuN}_{2}\right]^{+}:$787.39, found 787.24.

\section{1-bromododec-1-yn-3-one (4e)}




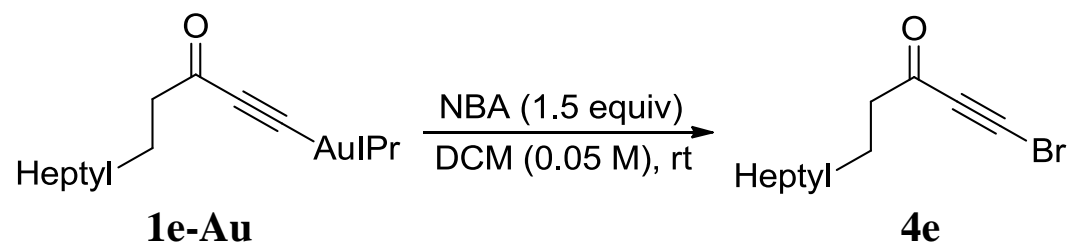

NBA (20.6 mg, 1.5 equiv) was added in one portion to a mixture of $\mathbf{1 e - A u}(0.1 \mathrm{mmol}, 76.6$ $\mathrm{mg})$ and DCM $(2 \mathrm{~mL})$. The above solution was stirred for 36 hours at room temperature and then concentrated under reduced pressure. The residue was purified by chromatography on silica gel to afford the desired product $4 \mathbf{e}(18.2 \mathrm{mg}, 70 \%) .{ }^{1} \mathrm{H}$ NMR $(500 \mathrm{MHz}$, Chloroform- $d$ ) $\delta 2.55(\mathrm{t}, J=7.4 \mathrm{~Hz}, 2 \mathrm{H}), 1.66(\mathrm{p}, J=7.2 \mathrm{~Hz}, 2 \mathrm{H}), 1.34-1.19$ $(\mathrm{m}, 12 \mathrm{H}), 0.88(\mathrm{t}, J=6.8 \mathrm{~Hz}, 3 \mathrm{H}) ;{ }^{13} \mathrm{C}$ NMR $\left(126 \mathrm{MHz}, \mathrm{CDCl}_{3}\right) \delta 186.57,80.01,56.77$, 45.37, 31.83, 29.34, 29.25, 29.21, 28.88, 23.84, 22.64, 14.08; IR (neat): 2955, 2927, 2855, 2178, 1680, 1466, 1136, 1098; $\mathrm{ESI}^{+}$calculated for $\left[\mathrm{C}_{12} \mathrm{H}_{19} \mathrm{KOBr}+\mathrm{MeOH}\right]^{+}: 329.05$, 331.05 , found $328.99,330.99$.

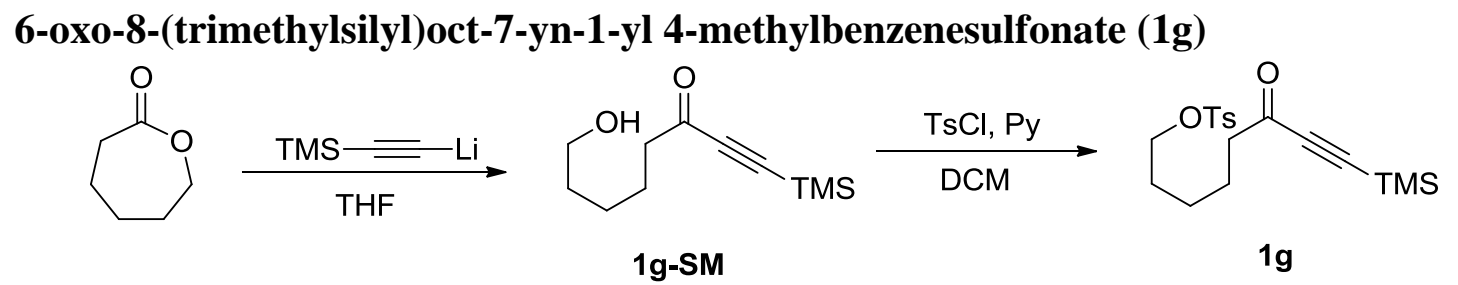

$\mathrm{BuLi}(22 \mathrm{mmol})$ was added dropwise to a mixture of trimethylsilyl acetylene $(2.9 \mathrm{~mL}, 22$ $\mathrm{mmol})$ and THF $(100 \mathrm{~mL})$ at $-78^{\circ} \mathrm{C}$. The mixture was allowed to stir for 30 minutes at $78^{\circ} \mathrm{C}$. Then $\varepsilon$-caprolactone $(2.2 \mathrm{~mL}, 20 \mathrm{mmol})$ was added in one portion. The mixture was allowed to stir for 90 minutes at $-78^{\circ} \mathrm{C}$ before it was quenched with $10 \% \mathrm{HCl}$ solution and extracted with ether. The combined organic extract was washed with brine, dried over anhydrous $\mathrm{MgSO}_{4}$, and concentrated under reduced pressure to give $\mathbf{1 g - S M}$ (3.2 g, crude), which was used without purification. $424 \mathrm{mg}(2 \mathrm{mmol})$ of the above crude product 1g-SM was disolved in $10 \mathrm{~mL}$ DCM. Pyridine $(0.65 \mathrm{~mL}, 8 \mathrm{mmol})$ and TsCl $(571 \mathrm{mg}, 3 \mathrm{mmol})$ were added in this order to the above DCM solution. The resulting mixture was allowed to stir at room temperature overnight. After the reaction finished, the reaction mixture was quenched with $1 \mathrm{M} \mathrm{HCl}$ solution $(12 \mathrm{~mL})$ and extracted with $\mathrm{Et}_{2} \mathrm{O}$. The combined organic extract was washed with brine, dried over anhydrous $\mathrm{MgSO}_{4}$, and concentrated under reduced pressure. The residue was purified by chromatography on silica gel to afford the desired product $1 \mathrm{~g}(659 \mathrm{mg}, 90 \%) .{ }^{1} \mathrm{H}$ NMR $(500 \mathrm{MHz}$, Chloroform- $d$ ) $\delta 7.78(\mathrm{~d}, J=8.3$ $\mathrm{Hz}, 2 \mathrm{H}), 7.45-7.31(\mathrm{~m}, 2 \mathrm{H}), 4.02(\mathrm{t}, J=6.4 \mathrm{~Hz}, 2 \mathrm{H}), 2.52(\mathrm{t}, J=7.3 \mathrm{~Hz}, 2 \mathrm{H}), 2.45(\mathrm{~s}$, $3 \mathrm{H}), 1.70-1.62(\mathrm{~m}, 2 \mathrm{H}), 1.62-1.58(\mathrm{~m}, 2 \mathrm{H}), 1.38-1.30(\mathrm{~m}, 2 \mathrm{H}), 0.24(\mathrm{~s}, 9 \mathrm{H}) ;{ }^{13} \mathrm{C} \mathrm{NMR}$ $\left(126 \mathrm{MHz}, \mathrm{CDCl}_{3}\right) \delta 187.21,144.72,133.13,129.82,127.86,101.89,97.94,70.15,44.88$, 28.61, 24.73, 23.07, 21.62, -0.78; IR (neat): 2959, 2148, 1676, 1598, 1360, 1252, 1189, 1177,847 ; $\mathrm{ESI}^{+}$calculated for $\left[\mathrm{C}_{18} \mathrm{H}_{26} \mathrm{NaSO}_{4} \mathrm{Si}\right]^{+}: 389.12$, found 389.03 .

\section{8-bromo-1-(trimethylsilyl)oct-1-yn-3-one (1h)}




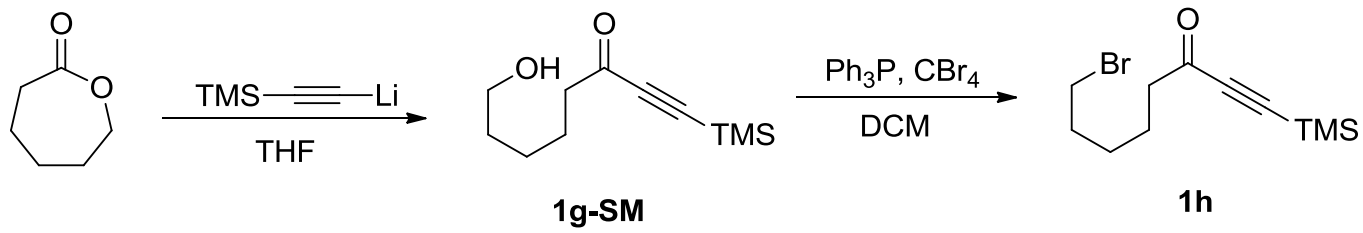

$\mathrm{Ph}_{3} \mathrm{P}(1.04 \mathrm{~g})$ and $\mathrm{CBr}_{4}(1.09 \mathrm{~g})$ were stirred in $\mathrm{DCM}(50 \mathrm{~mL})$ at $0^{\circ} \mathrm{C}$ for 17 minutes. Then, $551 \mathrm{mg}(2.6 \mathrm{mmol})$ of the crude $\mathbf{1 g - S M}$ was added to the above reaction mixture. The resulting solution was allowed to stir at $0^{\circ} \mathrm{C}$ for 1 hour. After the reaction finished, the reaction mixture was quenched with water and extracted with DCM. The combined organic extract was washed with brine, dried over anhydrous $\mathrm{MgSO}_{4}$, and concentrated under reduced pressure. The residue was purified by chromatography on silica gel to afford the desired product 1h. ${ }^{1} \mathrm{H}$ NMR (500 MHz, Chloroform- $d$ ) $\delta 3.40(\mathrm{t}, J=6.7 \mathrm{~Hz}, 2 \mathrm{H}), 2.58(\mathrm{t}$, $J=7.3 \mathrm{~Hz}, 2 \mathrm{H}), 1.88(\mathrm{dt}, J=14.9,6.9 \mathrm{~Hz}, 2 \mathrm{H}), 1.69(\mathrm{p}, J=7.4 \mathrm{~Hz}, 2 \mathrm{H}), 1.51-1.43(\mathrm{~m}$, $2 \mathrm{H}), 0.24(\mathrm{~s}, 9 \mathrm{H}) ;{ }^{13} \mathrm{C} \mathrm{NMR}\left(126 \mathrm{MHz}, \mathrm{CDCl}_{3}\right) \delta 187.38,101.92,97.93,44.96,33.35$, 32.42, 27.44, 22.97, -0.77; IR (neat): 2980, 2865, 2863, 2151, 1678, 1458, 1356, 1252, 1104,847 ; $\mathrm{ESI}^{+}$calculated for $\left[\mathrm{C}_{11} \mathrm{H}_{19} \mathrm{NaOBrSi}+\mathrm{MeOH}\right]^{+}: 329.05,331.05$, found 328.97 , 330.97.

\section{4-methyl-1-(trimethylsilyl)non-1-yn-3-one (1i)}

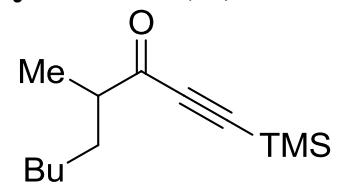

1i

Compound 1i was prepared following the general procedure B. ${ }^{1} \mathrm{H}$ NMR $(500 \mathrm{MHz}$, Chloroform- $d$ ) $\delta 2.54(\mathrm{~h}, J=6.9 \mathrm{~Hz}, 1 \mathrm{H}), 1.81-1.70(\mathrm{~m}, 1 \mathrm{H}), 1.46-1.36(\mathrm{~m}, 1 \mathrm{H}), 1.35-$ $1.23(\mathrm{~m}, 6 \mathrm{H}), 1.15(\mathrm{~d}, J=6.9 \mathrm{~Hz}, 3 \mathrm{H}), 0.88(\mathrm{t}, J=6.9 \mathrm{~Hz}, 3 \mathrm{H}), 0.24(\mathrm{~s}, 9 \mathrm{H}) ;{ }^{13} \mathrm{C} \mathrm{NMR}$ $\left(126 \mathrm{MHz}, \mathrm{CDCl}_{3}\right) \delta 192.04,101.19,98.47,48.28,32.65,31.71,26.59,22.40,15.84$, 13.97, -0.75; IR (neat): 2961, 2932, 2860, 2150, 1676, 1459, 1252, 847; $\mathrm{ESI}^{+}$calculated for $\left[\mathrm{C}_{13} \mathrm{H}_{24} \mathrm{NaOSi}\right]^{+}: 247.15$, found 247.09 .

\section{4,6-dimethyl-1-(trimethylsilyl)hept-1-yn-3-one (1j)}

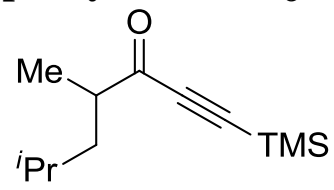

$1 \mathbf{j}$

Compound 1j was prepared following the general procedure B. ${ }^{1} \mathrm{H}$ NMR $(500 \mathrm{MHz}$, Chloroform- $d$ ) $\delta 2.63(\mathrm{~h}, J=7.0 \mathrm{~Hz}, 1 \mathrm{H}), 1.66(\mathrm{ddt}, J=32.0,13.4,7.0 \mathrm{~Hz}, 2 \mathrm{H}), 1.23(\mathrm{dt}$, $J=13.4,7.0 \mathrm{~Hz}, 1 \mathrm{H}), 1.15(\mathrm{~d}, J=6.9 \mathrm{~Hz}, 3 \mathrm{H}), 0.91(\mathrm{dd}, J=12.7,6.5 \mathrm{~Hz}, 6 \mathrm{H}), 0.25$ (s, $9 \mathrm{H}) ;{ }^{13} \mathrm{C} \mathrm{NMR}\left(126 \mathrm{MHz}, \mathrm{CDCl}_{3}\right) \delta 192.19,101.17,98.62,46.43,41.98,25.77,22.69$, 22.40, 16.36, -0.74; IR (neat): 2960, 2148, 1677, 1468, 1252, 1082, 847; ESI ${ }^{+}$calculated for $\left[\mathrm{C}_{12} \mathrm{H}_{22} \mathrm{NaOSi}+\mathrm{MeOH}\right]^{+}:$265.16, found 265.09.

\section{6-((tert-butyldiphenylsilyl)oxy)-4-methyl-1-(trimethylsilyl)hex-1-yn-3-one (1k)}




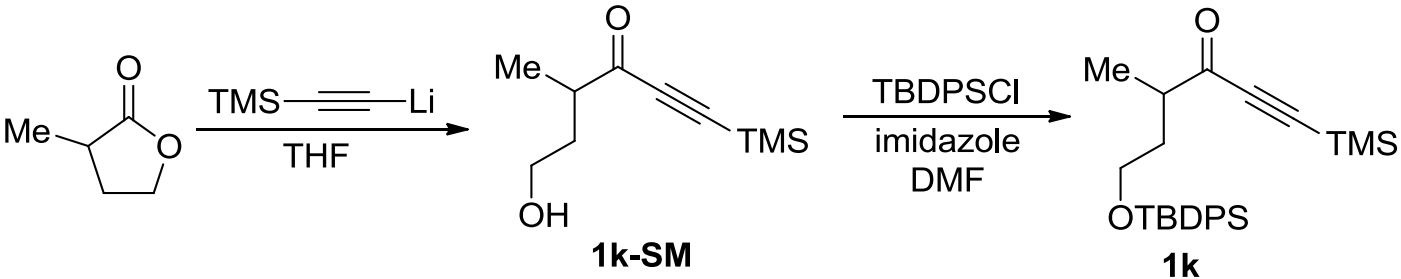

1k-SM was synthesized according to reported procedure (J. Org. Chem., 2012, 77, 3846). TBDPSCl $(0.11 \mathrm{~mL})$ was added to a solution of $\mathbf{1 k - S M}(0.35 \mathrm{mmol}, 70 \mathrm{mg})$, imidazole (71 $\mathrm{mg}$ ), and DMF (2mL). The resulting mixture was allowed to stir at room temperature overnight. After the reaction finished, the reaction mixture was quenched with $1 \mathrm{M} \mathrm{HCl}$ solution $(5 \mathrm{~mL})$ and extracted with $\mathrm{Et}_{2} \mathrm{O}$. The combined organic extract was washed with brine, dried over anhydrous $\mathrm{MgSO}_{4}$, and concentrated under reduced pressure. The residue was purified by chromatography on silica gel to afford the desired product $1 \mathbf{k} .{ }^{1} \mathrm{H}$ NMR (400 MHz, Chloroform- $d$ ) $\delta 7.67$ (dt, $J=8.0,1.8 \mathrm{~Hz}, 4 \mathrm{H}), 7.50-7.34(\mathrm{~m}, 6 \mathrm{H}), 3.77-3.60$ $(\mathrm{m}, 2 \mathrm{H}), 2.85(\mathrm{~h}, J=6.9 \mathrm{~Hz}, 1 \mathrm{H}), 2.15(\mathrm{ddd}, J=13.8,12.6,6.6 \mathrm{~Hz}, 1 \mathrm{H}), 1.64(\mathrm{dt}, J=14.0$, $6.0 \mathrm{~Hz}, 1 \mathrm{H}), 1.17(\mathrm{~d}, J=7.0 \mathrm{~Hz}, 3 \mathrm{H}), 1.06(\mathrm{~s}, 9 \mathrm{H}), 0.25(\mathrm{~s}, 9 \mathrm{H}) ;{ }^{13} \mathrm{C} \mathrm{NMR}(126 \mathrm{MHz}$, $\left.\mathrm{CDCl}_{3}\right) \delta 191.40,135.55,133.71,133.62,129.61,127.65,101.41,98.44,61.27,45.19$, 35.18, 26.81, 19.18, 15.70, -0.71; IR (neat): 3073, 3050, 2961, 2932, 2153, 2092, 1676, 1473, 1112, 846; $\mathrm{ESI}^{+}$calculated for $\left[\mathrm{C}_{26} \mathrm{H}_{36} \mathrm{NaO}_{2} \mathrm{Si}_{2}\right]^{+}: 459.20$, found 459.10 .

\section{4-(2-((tert-butyldiphenylsilyl)oxy)ethyl)-1-(trimethylsilyl)hept-1-yn-3-one (11)}
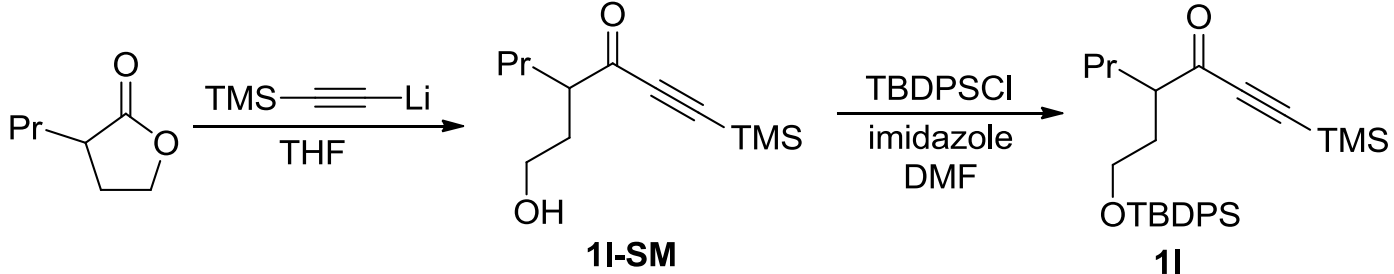

11 was synthesized using the same method as 1k. ${ }^{1} \mathrm{H}$ NMR $(500 \mathrm{MHz}$, Chloroform- $d$ ) $\delta$ $7.66(\mathrm{dt}, J=8.1,2.0 \mathrm{~Hz}, 4 \mathrm{H}), 7.46-7.34(\mathrm{~m}, 6 \mathrm{H}), 3.65(\mathrm{t}, J=6.2 \mathrm{~Hz}, 2 \mathrm{H}), 2.77(\mathrm{tt}, J=$ 8.2, $5.4 \mathrm{~Hz}, 1 \mathrm{H}), 2.07$ (ddt, $J=14.3,8.7,6.2 \mathrm{~Hz}, 1 \mathrm{H}), 1.78-1.64(\mathrm{~m}, 2 \mathrm{H}), 1.51-1.42(\mathrm{~m}$, $1 \mathrm{H}), 1.38-1.27(\mathrm{~m}, 2 \mathrm{H}), 1.05(\mathrm{~s}, 9 \mathrm{H}), 0.91(\mathrm{t}, J=7.3 \mathrm{~Hz}, 3 \mathrm{H}), 0.24(\mathrm{~s}, 9 \mathrm{H}) ;{ }^{13} \mathrm{C} \mathrm{NMR}$ $\left(126 \mathrm{MHz}, \mathrm{CDCl}_{3}\right) \delta 191.38,135.56,135.54,133.70,133.63,129.58,127.63,101.64$, 98.19, 61.62, 50.81, 33.81, 33.43, 26.80, 20.25, 19.16, 14.06, -0.71; IR (neat): 3073, 3050, 2959, 2932, 2858, 2153, 1674, 1473, 1428, 1252, 1112, 1092, 846; $\mathrm{ESI}^{+}$calculated for $\left[\mathrm{C}_{28} \mathrm{H}_{40} \mathrm{NaO}_{2} \mathrm{Si}_{2}\right]^{+}:$487.25, found 487.13.

(E)-methyl 6-oxo-5-propyl-8-(trimethylsilyl)oct-2-en-7-ynoate (1m)

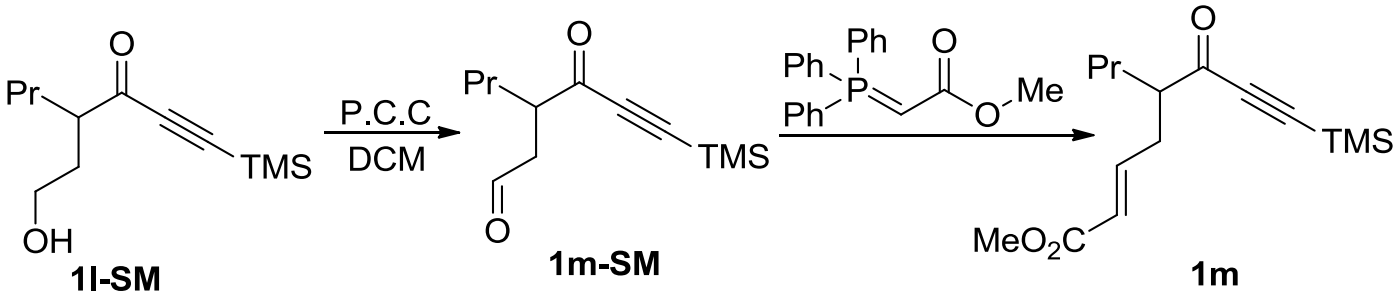

P.C.C (1.5 equiv, $259 \mathrm{mg}$ ) was added to a solution of $\mathbf{1 1 - S M}(0.8 \mathrm{mmol}, 200 \mathrm{mg})$ and DCM $(6 \mathrm{~mL})$. The resulting mixture was allowed to stir at room temperature overnight. After the 
reaction finished, the reaction mixture was first filtered through a short silica gel and then washed with $1 \mathrm{M} \mathrm{HCl}$ solution $(5 \mathrm{~mL})$ and extracted with DCM. The combined organic extract was washed with brine, dried over anhydrous $\mathrm{MgSO}_{4}$, and concentrated under reduced pressure to afford the crude $\mathbf{1 m - S M}$ (about $0.4 \mathrm{mmol}$ ), which was then dissolved in DCM (4 mL). To the above DCM solution was added methyl 2(triphenylphosphoranylidene)acetate $(0.44 \mathrm{mmol}, 147 \mathrm{mg})$. The resulting mixture was allowed to stir at room temperature overnight. After the reaction finished, the reaction mixture was quenched with water and extracted with DCM. The combined organic extract was washed with brine, dried over anhydrous $\mathrm{MgSO}_{4}$, and concentrated under reduced pressure. The residue was purified by chromatography on silica gel to afford the desired product $1 \mathrm{~m}(100 \mathrm{mg}, 90 \%) .{ }^{1} \mathrm{H}$ NMR $(500 \mathrm{MHz}$, Chloroform- $d) \delta 6.86(\mathrm{dt}, J=15.6,7.2$ $\mathrm{Hz}, 1 \mathrm{H}), 5.86(\mathrm{~d}, J=15.6 \mathrm{~Hz}, 1 \mathrm{H}), 3.72(\mathrm{~s}, 3 \mathrm{H}), 2.78-2.51(\mathrm{~m}, 2 \mathrm{H}), 2.37$ (dddd, $J=14.9$, 7.5, 5.8, $1.5 \mathrm{~Hz}, 1 \mathrm{H}), 1.73$ (dddd, $J=13.5,9.9,7.6,5.6 \mathrm{~Hz}, 1 \mathrm{H}), 1.56-1.45(\mathrm{~m}, 1 \mathrm{H}), 1.40$ $-1.25(\mathrm{~m}, 2 \mathrm{H}), 0.92(\mathrm{t}, J=7.3 \mathrm{~Hz}, 3 \mathrm{H}), 0.25(\mathrm{~s}, 9 \mathrm{H}) ;{ }^{13} \mathrm{C} \mathrm{NMR}\left(126 \mathrm{MHz}, \mathrm{CDCl}_{3}\right) \delta$ $189.74,166.55,145.45,123.10,101.13,99.61,52.59,51.47,33.26,32.99,20.04,13.99$, 0.80; IR (neat): 2960, 2934, 2875, 2150, 1728, 1675, 1253, 1143, 1044; $\mathrm{ESI}^{+}$calculated for $\left[\mathrm{C}_{15} \mathrm{H}_{24} \mathrm{NaO}_{3} \mathrm{Si}\right]^{+}:$303.14, found 303.05.

\section{4-ethyl-6-methyl-1-(trimethylsilyl)hept-1-yn-3-one (1n)}

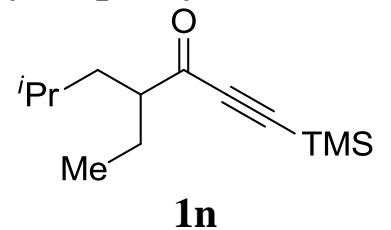

Compound 1n was prepared following the general procedure B. ${ }^{1} \mathrm{H}$ NMR $(500 \mathrm{MHz}$, Chloroform- $d$ ) $\delta 2.49(\mathrm{tt}, J=8.8,5.3 \mathrm{~Hz}, 1 \mathrm{H}), 1.75-1.63(\mathrm{~m}, 2 \mathrm{H}), 1.56$ (dddd, $J=21.5$, $13.4,10.1,5.9 \mathrm{~Hz}, 2 \mathrm{H}), 1.25$ (ddd, $J=13.5,8.2,5.4 \mathrm{~Hz}, 1 \mathrm{H}), 0.92-0.87$ (m, 9H), 0.24 (s, $9 \mathrm{H}) ;{ }^{13} \mathrm{C}$ NMR $\left(126 \mathrm{MHz}, \mathrm{CDCl}_{3}\right) \delta 192.23,101.30,98.36,53.93,40.38,26.01,25.06$, 23.10, 22.17, 11.56, -0.74; IR (neat): 2961, 2934, 2873, 2151, 2090, 1673, 1467, 1253, 1058,$847 ; \mathrm{ESI}^{+}$calculated for $\left[\mathrm{C}_{13} \mathrm{H}_{24} \mathrm{KOSi}\right]^{+}: 263.12$, found 263.07 .

1-cyclobutyl-3-(trimethylsilyl)prop-2-yn-1-one (10)

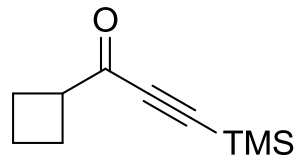

10

Compound 10 was prepared following the general procedure B. ${ }^{1} \mathrm{H}$ NMR $(500 \mathrm{MHz}$, Chloroform- $d$ ) $\delta 3.40-3.25(\mathrm{~m}, 1 \mathrm{H}), 2.41-2.28(\mathrm{~m}, 2 \mathrm{H}), 2.20(\mathrm{dtdd}, J=12.6,8.4,4.2$, $2.1 \mathrm{~Hz}, 2 \mathrm{H}), 1.99(\mathrm{dp}, J=11.1,8.6 \mathrm{~Hz}, 1 \mathrm{H}), 1.92-1.80(\mathrm{~m}, 1 \mathrm{H}), 0.24(\mathrm{~s}, 9 \mathrm{H}) ;{ }^{13} \mathrm{C} \mathrm{NMR}$ $\left(126 \mathrm{MHz} \mathrm{CDCl}_{3}\right) \delta 189.50,100.92,99.06,47.35,24.54,17.83,-0.73$; IR (neat): 2955, $2148,1757,1250,1134,845 ; \mathrm{ESI}^{+}$calculated for $\left[\mathrm{C}_{10} \mathrm{H}_{16} \mathrm{NaOSi}+\mathrm{MeOH}\right]^{+}: 235.11$, found 235.05 .

\section{1-(1-methylcyclopentyl)-3-(trimethylsilyl)prop-2-yn-1-one (1q)}




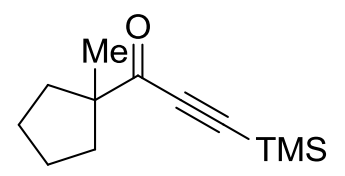

$1 \mathbf{q}$

Compound 1q was prepared following the general procedure B. ${ }^{1} \mathrm{H}$ NMR $(500 \mathrm{MHz}$, Chloroform- $d$ ) $\delta 2.24-2.13(\mathrm{~m}, 2 \mathrm{H}), 1.68(\mathrm{hd}, J=5.1,3.6 \mathrm{~Hz}, 4 \mathrm{H}), 1.47-1.39(\mathrm{~m}, 2 \mathrm{H})$, $1.24(\mathrm{~s}, 3 \mathrm{H}), 0.24(\mathrm{~s}, 9 \mathrm{H}) ;{ }^{13} \mathrm{C}$ NMR $\left(126 \mathrm{MHz}, \mathrm{CDCl}_{3}\right) \delta 193.42,100.61,98.79,55.85$, 36.90, 25.47, 23.87, -0.72; IR (neat): 2961, 2151, 1670, 1252, 1100, 845; ESI ${ }^{+}$calculated for $\left[\mathrm{C}_{12} \mathrm{H}_{20} \mathrm{NaOSi}+\mathrm{MeOH}\right]^{+}:$263.14, found 263.07.

1-cyclohexyl-3-(trimethylsilyl)prop-2-yn-1-one (1r)

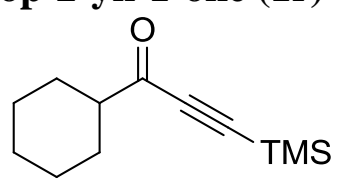

1r

Compound 1r was prepared following the general procedure B. ${ }^{1} \mathrm{H}$ NMR $(500 \mathrm{MHz}$, Chloroform- $d$ ) $\delta 2.38(\mathrm{tt}, J=11.1,3.6 \mathrm{~Hz}, 1 \mathrm{H}), 1.97(\mathrm{dq}, J=11.1,3.3,2.5 \mathrm{~Hz}, 2 \mathrm{H}), 1.78$ $(\mathrm{dt}, J=12.7,3.7 \mathrm{~Hz}, 2 \mathrm{H}), 1.70-1.62(\mathrm{~m}, 1 \mathrm{H}), 1.46-1.35(\mathrm{~m}, 2 \mathrm{H}), 1.35-1.13(\mathrm{~m}, 3 \mathrm{H})$, $0.24(\mathrm{~s}, 9 \mathrm{H}) ;{ }^{13} \mathrm{C}$ NMR $\left(126 \mathrm{MHz}, \mathrm{CDCl}_{3}\right) \delta 191.23,101.41,98.39,51.99,28.13,25.76$, 25.35, -0.72; IR (neat): 2933, 2856, 2151, 1673, 1451, 1252, 871; $\mathrm{ESI}^{+}$calculated for $\left[\mathrm{C}_{12} \mathrm{H}_{20} \mathrm{NaOSi}\right]^{+}:$263.14, found 263.07.

\section{1-(1-methylcyclohexyl)-3-(trimethylsilyl)prop-2-yn-1-one (1s)}

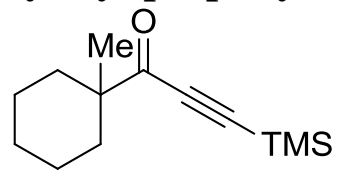

1s

Compound 1s was prepared following the general procedure B. ${ }^{1} \mathrm{H}$ NMR $(500 \mathrm{MHz}$, Chloroform- $d$ ) $\delta 2.11-1.98(\mathrm{~m}, 2 \mathrm{H}), 1.56-1.50(\mathrm{~m}, 2 \mathrm{H}), 1.49-1.25(\mathrm{~m}, 6 \mathrm{H}), 1.14(\mathrm{~s}$, $3 \mathrm{H}), 0.24(\mathrm{~s}, 9 \mathrm{H}) ;{ }^{13} \mathrm{C} \mathrm{NMR}\left(126 \mathrm{MHz}, \mathrm{CDCl}_{3}\right) \delta$ 194.14, 100.46, 98.49, 48.43, 34.38, 25.70, 24.77, 22.71, -0.71; IR (neat): 2962, 2934, 2855, 2149, 1669, 1450, 1252, 1080, 846; $\mathrm{ESI}^{+}$calculated for $\left[\mathrm{C}_{13} \mathrm{H}_{22} \mathrm{NaOSi}\right]^{+}: 245.13$, found 245.07 .

\section{1-(1,4-dimethylcyclohexyl)-3-(trimethylsilyl)prop-2-yn-1-one (1t)}

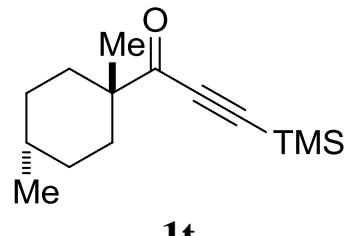

1t

Compound 1t was prepared following the general procedure B. ${ }^{1} \mathrm{H}$ NMR $(500 \mathrm{MHz}$, Chloroform- $d$ ) $\delta 1.74-1.66(\mathrm{~m}, 2 \mathrm{H}), 1.64-1.55(\mathrm{~m}, 4 \mathrm{H}), 1.35(\mathrm{ddq}, J=14.5,7.2,3.6 \mathrm{~Hz}$, $1 \mathrm{H}), 1.18(\mathrm{~s}, 3 \mathrm{H}), 1.16-1.08(\mathrm{~m}, 2 \mathrm{H}), 0.92(\mathrm{~d}, J=6.6 \mathrm{~Hz}, 3 \mathrm{H}), 0.24(\mathrm{~s}, 9 \mathrm{H}) ;{ }^{13} \mathrm{C} \mathrm{NMR}$ $\left(126 \mathrm{MHz} \mathrm{CDCl}_{3}\right) \delta 194.66,100.38,99.20,47.36,32.55,31.83,29.88,21.97,19.54$, - 
0.69; IR (neat): 2929, 2857, 2148, 2092, 1669, 1466, 866; $\mathrm{ESI}^{+}$calculated for $\left[\mathrm{C}_{14} \mathrm{H}_{24} \mathrm{KOSi}\right]^{+}: 275.12$, found 275.04 .

\section{1-(1-methylcycloheptyl)-3-(trimethylsilyl)prop-2-yn-1-one (1u)}

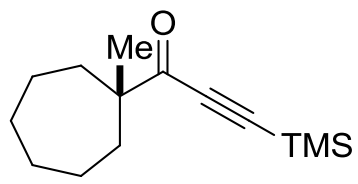

1u

Compound 1u was prepared following the general procedure B. ${ }^{1} \mathrm{H}$ NMR $(500 \mathrm{MHz}$, Chloroform- $d$ ) $\delta 2.12(\mathrm{ddd}, J=13.3,6.3,2.7 \mathrm{~Hz}, 2 \mathrm{H}), 1.58-1.38(\mathrm{~m}, 10 \mathrm{H}), 1.16(\mathrm{~s}, 3 \mathrm{H})$, 0.24 (s, 9H); ${ }^{13} \mathrm{C}$ NMR $\left(126 \mathrm{MHz}, \mathrm{CDCl}_{3}\right) \delta 194.19,100.78,98.80,51.29,36.50,30.24$, 26.13, 23.43, -0.72; IR (neat): 2962, 2927, 2148, 2089, 1669, 1466, 1251, 1041, 845; ESI ${ }^{+}$ calculated for $\left[\mathrm{C}_{14} \mathrm{H}_{24} \mathrm{NaOSi}\right]^{+}: 259.15$, found 259.09 .

\section{cis-3-(2-((trimethylsilyl)ethynyl)-1,3-dithian-2-yl)cyclohexanol (1w-SM) trans-3-(2-((trimethylsilyl)ethynyl)-1,3-dithian-2-yl)cyclohexanol (1x-SM)}

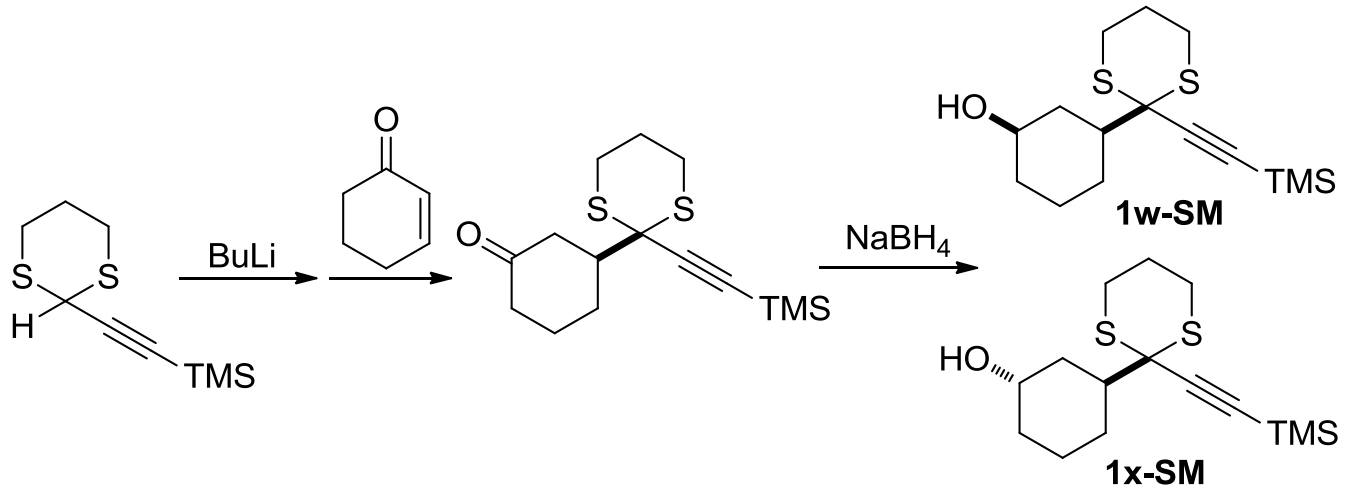

BuLi (3 mL, 2.5 M) was added dropwise to a mixture of ((1,3-dithian-2-yl)ethynyl)trimethylsilane $(1.4 \mathrm{~g}, 6.5 \mathrm{mmol})$ and THF $(50 \mathrm{~mL})$ at $-78^{\circ} \mathrm{C}$. The mixture was allowed to stir for 60 minutes at $-78^{\circ} \mathrm{C}$. Then HMPA $(2.3 \mathrm{~mL}, 2$ equiv) was added in one portion. The mixture was allowed to stir for 30 minutes at $-78^{\circ} \mathrm{C}$, then cyclohexenone $(0.6 \mathrm{~mL}, 6.5$ $\mathrm{mmol}$ ) was added in one portion to the above reaction mixture. The reaction was allowed to warm up to room temperature within four hours. After the reaction finished, the reaction mixture was quenched with saturated ammonium chloride solution $(20 \mathrm{~mL})$ and extracted with $\mathrm{Et}_{2} \mathrm{O}$. The combined organic extract was washed with brine, dried over anhydrous $\mathrm{MgSO}_{4}$, and concentrated under reduced pressure. The residue was directly dissolved in ethanol $(100 \mathrm{~mL}) . \mathrm{NaBH}_{4}(228 \mathrm{mg}, 6 \mathrm{mmol})$ was then added to the above ethanol solution at $0^{\circ} \mathrm{C}$. The resulting mixture was allowed to stir at the same temperature for 10 minutes. After the reaction finished, the reaction mixture was quenched with saturated ammonium chloride solution $(20 \mathrm{~mL})$ and extracted with $\mathrm{Et}_{2} \mathrm{O}$. The combined organic extract was washed with brine, dried over anhydrous $\mathrm{MgSO}_{4}$, and concentrated under reduced pressure. The residue was purified by chromatography on silica gel to afford the desired product $1 \mathrm{w}$ SM $(5 \mathrm{mmol}, 1.57 \mathrm{~g})$ and 1x-SM (1 mmol, $315 \mathrm{mg})$. 1w-SM: ${ }^{1} \mathrm{H}$ NMR $(500 \mathrm{MHz}$, Chloroform- $d$ ) $\delta 3.62(\mathrm{tt}, J=10.9,4.2 \mathrm{~Hz}, 1 \mathrm{H}), 3.33(\mathrm{ddd}, J=14.2,12.8,2.5 \mathrm{~Hz}, 2 \mathrm{H}), 2.87$ $-2.76(\mathrm{~m}, 2 \mathrm{H}), 2.52-2.39(\mathrm{~m}, 1 \mathrm{H}), 2.14(\mathrm{dddd}, J=12.9,6.8,5.0,3.3 \mathrm{~Hz}, 2 \mathrm{H}), 2.02-1.96$ 
$(\mathrm{m}, 1 \mathrm{H}), 1.92-1.83(\mathrm{~m}, 2 \mathrm{H}), 1.83-1.75(\mathrm{~m}, 1 \mathrm{H}), 1.37(\mathrm{td}, J=12.2,10.9 \mathrm{~Hz}, 1 \mathrm{H}), 1.33-$ $1.14(\mathrm{~m}, 3 \mathrm{H}), 0.22(\mathrm{~s}, 9 \mathrm{H}) ;{ }^{13} \mathrm{C} \mathrm{NMR}\left(126 \mathrm{MHz}, \mathrm{CDCl}_{3}\right) \delta 102.62,93.04,70.68,51.62$, 45.84, 37.41, 35.30, 28.68, 26.99, 25.97, 23.50, 0.20; IR (neat): 2936, 2904, 2861, 2156, $1451,1249,1054,843$; $\mathrm{ESI}^{+}$calculated for $\left[\mathrm{C}_{15} \mathrm{H}_{26} \mathrm{NaOS}_{2} \mathrm{Si}\right]^{+}: 337.11$, found 337.02 . 1xSM: ${ }^{1} \mathrm{H}$ NMR $(500 \mathrm{MHz}$, Chloroform- $d$ ) $\delta 4.24(\mathrm{p}, J=2.9 \mathrm{~Hz}, 1 \mathrm{H}), 3.33$ (dddd, $J=14.3$, 12.8, 2.6, $1.4 \mathrm{~Hz}, 2 \mathrm{H}), 2.81$ (dtd, $J=14.3,3.2,1.7 \mathrm{~Hz}, 2 \mathrm{H}), 2.34-2.17$ (m, 2H), 2.13 (dtd, $J=13.6,4.2,2.1 \mathrm{~Hz}, 1 \mathrm{H}), 1.87-1.67(\mathrm{~m}, 3 \mathrm{H}), 1.62(\mathrm{ddt}, J=13.8,9.0,2.5 \mathrm{~Hz}, 2 \mathrm{H}), 1.50$ - $1.30(\mathrm{~m}, 3 \mathrm{H}), 0.22(\mathrm{~s}, 9 \mathrm{H}) ;{ }^{13} \mathrm{C} \mathrm{NMR}\left(126 \mathrm{MHz}, \mathrm{CDCl}_{3}\right) \delta 102.88,92.89,66.52,52.17$, 41.03, 34.73, 32.24, 28.61, 28.59, 27.77, 26.00, 19.81, 0.23; IR (neat): 2932, 2904, 2153 , $1428,1248,843 ; \mathrm{ESI}^{+}$calculated for $\left[\mathrm{C}_{15} \mathrm{H}_{26} \mathrm{NaOS}_{2} \mathrm{Si}\right]^{+}: 337.11$, found 337.02 .

\section{1-(3-((tert-butyldiphenylsilyl)oxy)cyclohexyl)-3-(trimethylsilyl)prop-2-yn-1-one (1w)}

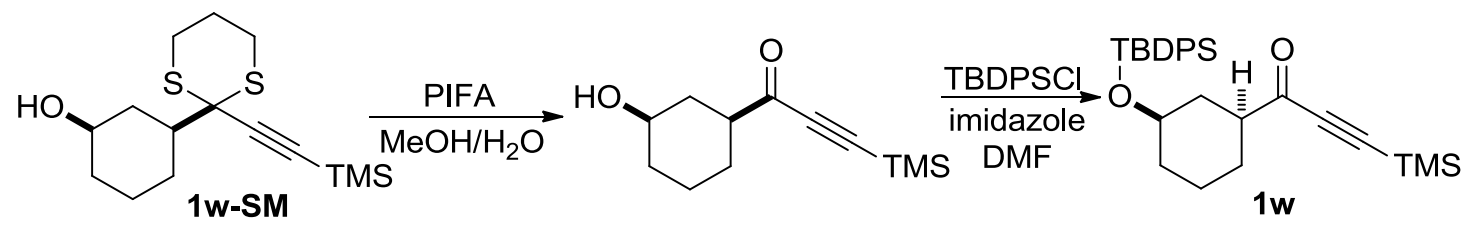

[Bis(trifluoroacetoxy)iodo]benzene (255 $\mathrm{mg}, 1.1$ equiv) was added portionwise to a mixture of 1w-SM (170 mg, $0.54 \mathrm{mmol})$, water $(1 \mathrm{~mL})$, and $\mathrm{MeOH}(10 \mathrm{~mL})$ at $0^{\circ} \mathrm{C}$. The mixture was allowed to stir for 10 minutes at $0^{\circ} \mathrm{C}$. Then the reaction mixture was quenched with saturated ammonium chloride solution $(20 \mathrm{~mL})$ and extracted with $\mathrm{Et}_{2} \mathrm{O}$. The combined organic extract was washed with saturated $\mathrm{NaHCO}_{3}$, brine, dried over anhydrous $\mathrm{MgSO}_{4}$, and concentrated under reduced pressure. The concentrated residue was directly dissolved in DMF (4 mL). Imidazole $(73 \mathrm{mg}, 1.08 \mathrm{mmol})$ and TBDPSCl (0.14 mL, 0.54 mmol) was then added to the above DMF solution at room temperature. The resulting mixture was allowed to stir at the same temperature overnight. After the reaction finished, the reaction mixture was quenched with saturated ammonium chloride solution $(20 \mathrm{~mL})$ and extracted with $\mathrm{Et}_{2} \mathrm{O}$. The combined organic extract was washed with brine, dried over anhydrous $\mathrm{MgSO}_{4}$, and concentrated under reduced pressure. The residue was purified by chromatography on silica gel to afford the desired product $\mathbf{1 w}(148 \mathrm{mg}, 60 \%) .{ }^{1} \mathrm{H} \mathrm{NMR}$ $(500 \mathrm{MHz}$, Chloroform- $d$ ) $\delta 7.70-7.65(\mathrm{~m}, 4 \mathrm{H}), 7.45-7.40(\mathrm{~m}, 2 \mathrm{H}), 7.37$ (ddd, $J=8.3$, 6.4, $1.5 \mathrm{~Hz}, 4 \mathrm{H}), 3.63$ (tt, $J=10.4,4.0 \mathrm{~Hz}, 1 \mathrm{H}), 2.27$ (tt, $J=11.7,3.6 \mathrm{~Hz}, 1 \mathrm{H}), 2.18$ (dtd, $J=11.8,3.8,1.9 \mathrm{~Hz}, 1 \mathrm{H}), 1.89-1.81(\mathrm{~m}, 1 \mathrm{H}), 1.76(\mathrm{ddt}, J=13.8,7.3,3.7 \mathrm{~Hz}, 2 \mathrm{H}), 1.60$ $-1.49(\mathrm{~m}, 1 \mathrm{H}), 1.33-1.20(\mathrm{~m}, 2 \mathrm{H}), 1.15-1.07(\mathrm{~m}, 1 \mathrm{H}), 1.06(\mathrm{~s}, 9 \mathrm{H}), 0.24(\mathrm{~s}, 9 \mathrm{H}) ;{ }^{13} \mathrm{C}$ NMR $\left(126 \mathrm{MHz}, \mathrm{CDCl}_{3}\right) \delta 189.62,135.75,135.73,134.54,134.31,129.57,129.54$, $127.53,127.50,101.20,98.81,71.36,50.62$, 37.06, 35.12, 27.08, 26.95, 23.04, 19.13, 0.72; IR (neat): 3072, 3053, 2934, 2858, 1673, 1428, 1252, 1111, 846, 702; ESI ${ }^{+}$calculated for $\left[\mathrm{C}_{28} \mathrm{H}_{38} \mathrm{NaO}_{2} \mathrm{Si}_{2}\right]^{+}$: 485.23, found 485.12.

\section{1-(3-((tert-butyldiphenylsilyl)oxy)cyclohexyl)-3-(trimethylsilyl)prop-2-yn-1-one (1x)}

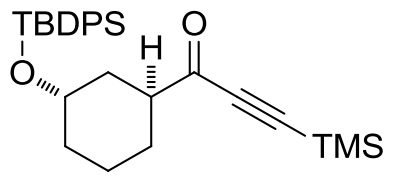

1x 
Compound $1 \mathbf{x}$ was synthesized using the same method as $1 \mathbf{w} .{ }^{1} \mathrm{H}$ NMR $(500 \mathrm{MHz}$, Chloroform- $d$ ) $\delta 7.67(\mathrm{dt}, J=8.0,1.2 \mathrm{~Hz}, 4 \mathrm{H}), 7.45-7.40(\mathrm{~m}, 2 \mathrm{H}), 7.40-7.35(\mathrm{~m}, 4 \mathrm{H})$, 4.14 (s, $1 \mathrm{H}), 3.03$ (tt, $J=11.4,3.5 \mathrm{~Hz}, 1 \mathrm{H}), 2.09-1.97$ (m, 2H), 1.88 (dddd, $J=16.3,12.8$, 8.1, $3.4 \mathrm{~Hz}, 1 \mathrm{H}), 1.61-1.52(\mathrm{~m}, 2 \mathrm{H}), 1.46-1.33(\mathrm{~m}, 2 \mathrm{H}), 1.27-1.19(\mathrm{~m}, 1 \mathrm{H}), 1.10(\mathrm{~s}$, 9H), 0.24 (s, 9H); ${ }^{13} \mathrm{C}$ NMR (126 MHz, $\left.\mathrm{CDCl}_{3}\right) \delta 191.35,135.74,135.69,134.44,134.06$, 129.63, 129.56, 127.59, 127.52, 101.38, 98.31, 67.25, 46.97, 35.23, 32.56, 27.47, 27.04, 19.61, 19.34, -0.70; IR (neat): 2966, 2930, 2858, 2091, 1643, 1428, 1251, 1105; ESI ${ }^{+}$ calculated for $\left[\mathrm{C}_{28} \mathrm{H}_{38} \mathrm{NaO}_{2} \mathrm{Si}_{2}\right]^{+}: 485.23$, found 485.12 .

General procedure C: Gold-catalyzed cyclopentenones synthesis via intramolecular insertions into unactivated $\mathrm{C}\left(\mathrm{sp}^{3}\right)$-H bonds
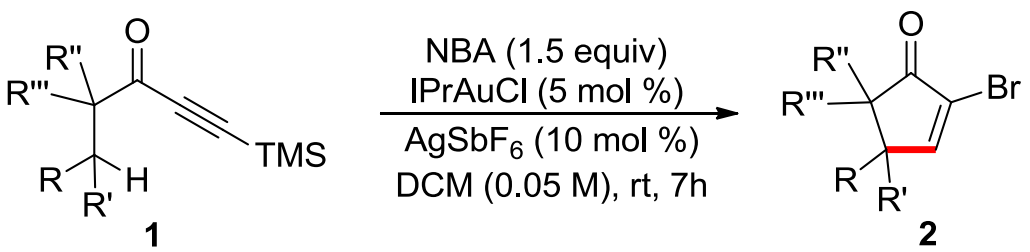

NBA (1.5 equiv, $62 \mathrm{mg}), \operatorname{IPrAuCl}(5 \mathrm{~mol} \%, 9.3 \mathrm{mg})$, and $\mathrm{AgSbF}_{6}(10 \mathrm{~mol} \%, 10.3 \mathrm{mg})$ were added in this order to a mixture of ynone $\mathbf{1}(0.3 \mathrm{mmol})$ and DCM $(6 \mathrm{~mL})$ in a vial at room temperature. The reaction mixture was stirred at room temperature for 7 hours. Upon completion, the mixture was concentrated and the residue was purified by chromatography on silica gel (eluent: hexanes/ethyl acetate) to afford the desired product 2.

General procedure D: Gold-catalyzed cyclopentenones synthesis via intramolecular insertions into unactivated $\mathrm{C}\left(\mathrm{sp}^{3}\right)-\mathrm{H}$ bonds
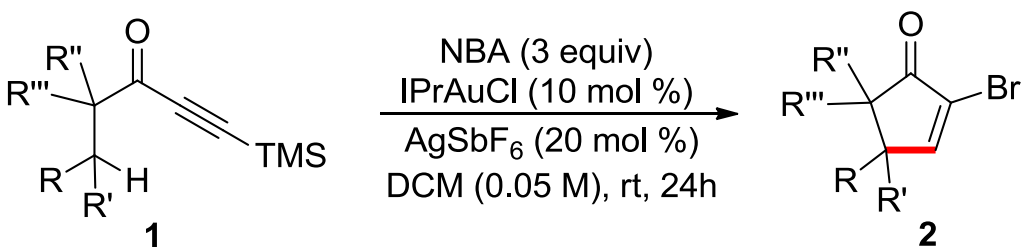

NBA (3 equiv, $124 \mathrm{mg}$ ), $\mathrm{IPrAuCl}(10 \mathrm{~mol} \%, 18.6 \mathrm{mg})$, and $\mathrm{AgSbF}_{6}(20 \mathrm{~mol} \%, 20.6 \mathrm{mg})$ were added in this order to a mixture of ynone $\mathbf{1}(0.3 \mathrm{mmol})$ and DCM $(6 \mathrm{~mL})$ in a vial at room temperature. The reaction mixture was stirred at room temperature for 24 hours. Upon completion, the mixture was concentrated and the residue was purified by chromatography on silica gel (eluent: hexanes/ethyl acetate) to afford the desired product 2.

General procedure E: Gold-catalyzed cyclopentenones synthesis via intramolecular insertions into unactivated $\mathrm{C}\left(\mathrm{sp}^{3}\right)-\mathrm{H}$ bonds
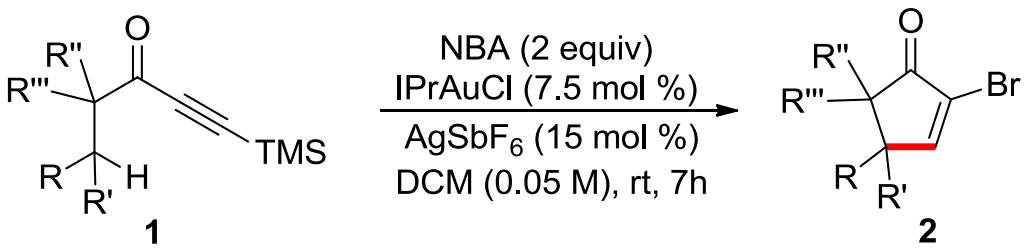
NBA (2 equiv, $82.2 \mathrm{mg}$ ), $\operatorname{IPrAuCl}(7.5 \mathrm{~mol} \%, 14.4 \mathrm{mg})$, and $\mathrm{AgSbF}_{6}(15 \mathrm{~mol} \%, 16.2 \mathrm{mg}$ ) were added in this order to a mixture of ynone $\mathbf{1}(0.3 \mathrm{mmol})$ and DCM $(6 \mathrm{~mL})$ in a vial at room temperature. The reaction mixture was stirred at room temperature for 7 hours. Upon completion, the mixture was concentrated and the residue was purified by chromatography on silica gel (eluent: hexanes/ethyl acetate) to afford the desired product $\mathbf{2}$.

\section{2-bromo-5,5-dimethyl-4-pentylcyclopent-2-enone (2a)}

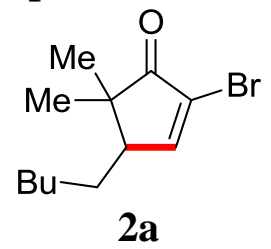

Compound 2a was prepared in $95 \%$ yield $(74.1 \mathrm{mg})$ using $1 \mathbf{a}(0.3 \mathrm{mmol})$ as the substrate according to the general procedure C. ${ }^{1} \mathrm{H}$ NMR $(500 \mathrm{MHz}$, Chloroform- $d) \delta 7.68(\mathrm{~d}, J=$ $2.7 \mathrm{~Hz}, 1 \mathrm{H}$ ), 2.49 (ddd, $J=9.6,5.7,2.7 \mathrm{~Hz}, 1 \mathrm{H}), 1.60-1.55$ (m, $1 \mathrm{H}), 1.49$ (ddtd, $J=12.4$, 8.0, 4.2, 3.6, $2.4 \mathrm{~Hz}, 1 \mathrm{H}), 1.42-1.24(\mathrm{~m}, 6 \mathrm{H}), 1.16(\mathrm{~s}, 3 \mathrm{H}), 1.04(\mathrm{~s}, 3 \mathrm{H}), 0.93-0.87(\mathrm{~m}$, $3 \mathrm{H}) ;{ }^{13} \mathrm{C}$ NMR $\left(126 \mathrm{MHz}, \mathrm{CDCl}_{3}\right) \delta 206.59,162.84,123.32,52.62,46.18,31.88,29.67$, 27.63, 25.47, 22.50, 20.94, 13.99; IR (neat): 3060, 2959, 2931, 2859, 1726, 1589, 1466, 842; $\mathrm{ESI}^{+}$calculated for $\left[\mathrm{C}_{12} \mathrm{H}_{29} \mathrm{NaOBr}\right]^{+}: 281.05,283.05$, found 280.98, 282.98.

\section{2-bromo-4-isopropyl-5,5-dimethylcyclopent-2-enone (2b)}

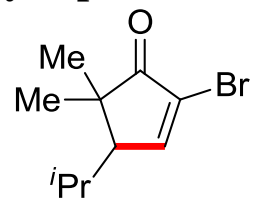

2b

Compound $\mathbf{2 b}$ was prepared in $91 \%$ yield $(63.1 \mathrm{mg})$ using $\mathbf{1 b}(0.3 \mathrm{mmol})$ as the substrate according to the general procedure C. ${ }^{1} \mathrm{H}$ NMR $(500 \mathrm{MHz}$, Chloroform- $d) \delta 7.71(\mathrm{~d}, J=$ $2.7 \mathrm{~Hz}, 1 \mathrm{H}), 2.31$ (dd, $J=7.0,2.8 \mathrm{~Hz}, 1 \mathrm{H}), 1.89(\mathrm{dq}, J=13.4,6.7 \mathrm{~Hz}, 1 \mathrm{H}), 1.19$ (s, 3H), $1.13(\mathrm{~s}, 3 \mathrm{H}), 1.10(\mathrm{~d}, J=6.6 \mathrm{~Hz}, 3 \mathrm{H}), 0.92(\mathrm{~d}, J=6.6 \mathrm{~Hz}, 3 \mathrm{H}) ;{ }^{13} \mathrm{C}$ NMR $(126 \mathrm{MHz}$, $\left.\mathrm{CDCl}_{3}\right) \delta 206.40,161.37,123.50,59.49,46.55,27.99,26.79,23.11,20.70,20.56$; IR (neat): 3068, 2965, 2933, 2873, 1726, 1590, 1463, 928, 840; $\mathrm{ESI}^{+}$calculated for $\left[\mathrm{C}_{10} \mathrm{H}_{15} \mathrm{NaOBr}\right]^{+}:$253.02, 255.02, found 252.96, 254.96.

\section{2-bromo-5,5-dimethyl-4-phenylcyclopent-2-enone (2c) \& 5-benzyl-2-bromo-5- methylcyclopent-2-enone (2c')}

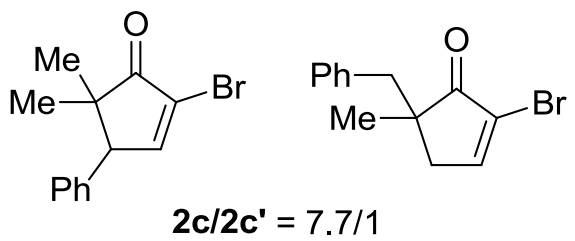

Compound $\mathbf{2 c} / \mathbf{2} \mathbf{c}$ ' was prepared in $72 \%$ yield $(57.2 \mathrm{mg})$ using $\mathbf{1 c}(0.3 \mathrm{mmol})$ as the substrate according to the general procedure $\mathbf{C}$. The ratio of $\mathbf{2} \mathbf{c} / \mathbf{2} \mathbf{c}^{\prime}$ is $7.7 / 1$ according to ${ }^{1} \mathrm{H}$ NMR. ${ }^{1} \mathrm{H}$ NMR $(500 \mathrm{MHz}$, Chloroform- $d$ ) (major) $\delta 7.76(\mathrm{~d}, J=2.7 \mathrm{~Hz}, 1 \mathrm{H}), 7.38-7.28$ (m, 3H), $7.08-7.04(\mathrm{~m}, 2 \mathrm{H}), 3.85(\mathrm{~d}, J=2.8 \mathrm{~Hz}, 1 \mathrm{H}), 1.33(\mathrm{~s}, 3 \mathrm{H}), 0.63(\mathrm{~s}, 3 \mathrm{H}) ;{ }^{1} \mathrm{H}$ NMR $(500 \mathrm{MHz}$, Chloroform- $d$ ) (selected peaks for minor) $\delta 7.52(\mathrm{t}, J=3.0 \mathrm{~Hz}, 1 \mathrm{H}), 3.01(\mathrm{~d}, J$ 
$=13.4 \mathrm{~Hz}, 1 \mathrm{H}), 2.74(\mathrm{dd}, J=19.1,3.0 \mathrm{~Hz}, 1 \mathrm{H}), 2.67(\mathrm{~d}, J=13.4 \mathrm{~Hz}, 1 \mathrm{H}), 2.30(\mathrm{dd}, J=$ 19.1, 3.0 Hz, 1H), 1.22 (s, 3H); ${ }^{13} \mathrm{C}$ NMR $\left(126 \mathrm{MHz}, \mathrm{CDCl}_{3}\right) \delta 206.19,206.12,160.51$, $159.78,137.82,136.83,129.89,128.65,128.39,128.24,127.60,126.76,124.57,124.23$, 58.30, 47.48, 47.02, 43.69, 40.60, 25.80, 24.62, 22.74; IR (neat): 3063, 3027, 2970, 2928, 1726, 1591, 1496, 1453, 1382, 840, 703; $\mathrm{ESI}^{+}$calculated for $\left[\mathrm{C}_{13} \mathrm{H}_{13} \mathrm{NaOBr}\right]^{+}: 287.00$, 289.00, found 286.94, 288.94 .

\section{8,9-dibromo-6,6-dimethyl-5H-benzo[7]annulen-7(6H)-one (3c)}

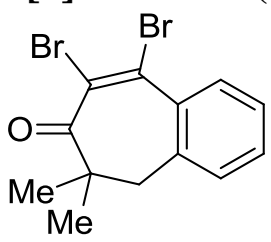

$3 c$

Compound 3c was prepared in $7 \%$ yield $(7.0 \mathrm{mg})$ using $\mathbf{1 c}(0.3 \mathrm{mmol})$ as the substrate according to the general procedure $\mathbf{C}$. ${ }^{1} \mathrm{H}$ NMR $(500 \mathrm{MHz}$, Chloroform- $d$ ) $\delta 7.62$ (dd, $J=$ 7.7, $1.5 \mathrm{~Hz}, 1 \mathrm{H}), 7.32(\mathrm{td}, J=7.6,1.6 \mathrm{~Hz}, 1 \mathrm{H}), 7.29-7.26(\mathrm{~m}, 1 \mathrm{H}), 7.14(\mathrm{dd}, J=7.3,1.5$ $\mathrm{Hz}, 1 \mathrm{H}), 2.95$ (s, 2H), $1.19(\mathrm{~s}, 6 \mathrm{H}) ;{ }^{13} \mathrm{C}$ NMR $\left(126 \mathrm{MHz}, \mathrm{CDCl}_{3}\right) \delta 202.88,136.14,136.00$, 130.75, 129.88, 129.55, 128.09, 127.53, 122.60, 55.89, 43.98, 25.00; IR (neat): 3068, 3027, $2963,2929,1701,1383,1101,755$; $\mathrm{ESI}^{+}$calculated for $\left[\mathrm{C}_{13} \mathrm{H}_{12} \mathrm{Br} 2 \mathrm{NaO}+\mathrm{MeOH}\right]^{+}: 396.94$, 398.94, 400.94, found 396.85, 398.86, 400.85 .

\section{2-bromo-4,4,5,5-tetramethylcyclopent-2-enone (2d)}

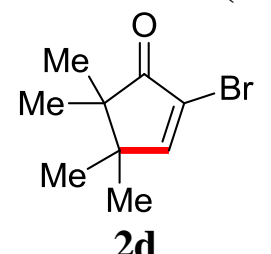

Compound $2 \mathbf{d}$ was prepared in $79 \%$ yield $(51.2 \mathrm{mg})$ using $\mathbf{1 d}(0.3 \mathrm{mmol})$ as the substrate according to the general procedure C. ${ }^{1} \mathrm{H}$ NMR $(500 \mathrm{MHz}$, Chloroform- $d) \delta 7.47(\mathrm{~s}, 1 \mathrm{H})$, $1.10(\mathrm{~s}, 6 \mathrm{H}), 1.07(\mathrm{~s}, 6 \mathrm{H}) ;{ }^{13} \mathrm{C} \mathrm{NMR}\left(126 \mathrm{MHz}, \mathrm{CDCl}_{3}\right) \delta 206.68,168.31,121.31,50.08$, 47.09, 24.79, 22.19, 22.18; IR (neat): 3053, 2973, 1725, 1591, 837, 743; ESI $^{+}$calculated for $\left[\mathrm{C}_{9} \mathrm{H}_{13} \mathrm{NaOBr}+\mathrm{MeOH}\right]^{+}:$271.03, 273.03, found 270.97, 272.97 .

\section{2-bromo-4-heptylcyclopent-2-enone (2e)}

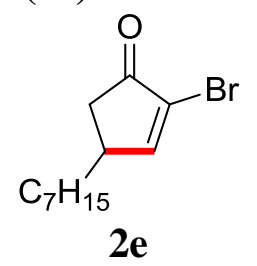

Compound $\mathbf{2 e}$ was prepared in $74 \%$ yield $(57.6 \mathrm{mg})$ using $1 \mathbf{e}(0.3 \mathrm{mmol})$ as the substrate according to the general procedure $\mathbf{C} .{ }^{1} \mathrm{H}$ NMR $(500 \mathrm{MHz}$, Chloroform- $d) \delta 7.71(\mathrm{~d}, J=$ $2.8 \mathrm{~Hz}, 1 \mathrm{H}), 2.94-2.86(\mathrm{~m}, 1 \mathrm{H}), 2.68(\mathrm{dd}, J=18.9,6.3 \mathrm{~Hz}, 1 \mathrm{H}), 2.19-2.10(\mathrm{~m}, 1 \mathrm{H}), 1.57$ $(\mathrm{ddd}, J=15.0,7.9,4.2 \mathrm{~Hz}, 1 \mathrm{H}), 1.48-1.20(\mathrm{~m}, 11 \mathrm{H}), 0.88(\mathrm{t}, J=6.8 \mathrm{~Hz}, 3 \mathrm{H}) ;{ }^{13} \mathrm{C} \mathrm{NMR}$ (126 MHz, $\left.\mathrm{CDCl}_{3}\right) \delta 201.26,165.65,125.41,40.53,39.44,34.63,31.73,29.44,29.09$, 
27.29, 22.59, 14.04; IR (neat): 3063, 2958, 2926, 2855, 1725, 1588, 1465, 923; ESI ${ }^{+}$ calculated for $\left[\mathrm{C}_{12} \mathrm{H}_{19} \mathrm{BrNaO}+\mathrm{MeOH}\right]^{+}: 313.08,315.08$, found 313.01, 315.01.

2-bromo-4-(((tert-butyldiphenylsilyl)oxy)methyl)cyclopent-2-enone (2f)

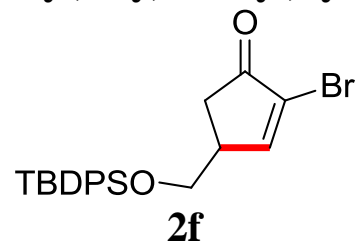

Compound $2 \mathbf{f}$ was prepared in $51 \%$ yield $(65.6 \mathrm{mg})$ using $\mathbf{1 f}(0.3 \mathrm{mmol})$ as the substrate according to the general procedure D. ${ }^{1} \mathrm{H}$ NMR $(500 \mathrm{MHz}$, Chloroform- $d$ ) $\delta 7.63-7.59$ (m, 5H), $7.47-7.43(\mathrm{~m}, 2 \mathrm{H}), 7.42-7.36(\mathrm{~m}, 4 \mathrm{H}), 3.77(\mathrm{dd}, J=10.1,5.1 \mathrm{~Hz}, 1 \mathrm{H}), 3.70$ (dd, $J=10.1,6.1 \mathrm{~Hz}, 1 \mathrm{H}), 3.09$ (dddd, $J=8.7,6.6,4.9,2.3 \mathrm{~Hz}, 1 \mathrm{H}$ ), 2.58 (dd, $J=18.8,6.5$ $\mathrm{Hz}, 1 \mathrm{H}), 2.33(\mathrm{dd}, J=18.8,2.0 \mathrm{~Hz}, 1 \mathrm{H}), 1.04(\mathrm{~s}, 9 \mathrm{H}) ;{ }^{13} \mathrm{C} \mathrm{NMR}\left(126 \mathrm{MHz}, \mathrm{CDCl}_{3}\right) \delta$ 200.86, 162.81, 135.58, 135.49, 132.98, 132.90, 129.97, 129.93, 127.84, 127.83, 126.73, 64.80, 42.97, 35.87, 26.77, 19.18; IR (neat): 3070, 3050, 2930, 2858, 1724, 1589, 1427, 1112,$741 ; \mathrm{ESI}^{+}$calculated for $\left[\mathrm{C}_{22} \mathrm{H}_{25} \mathrm{NaBrO}_{2} \mathrm{Si}^{+}:\right.$: 453.07, 451.07, found 450.97, 452.97 .

\section{3-(3-bromo-4-oxocyclopent-2-en-1-yl)propyl 4-methylbenzenesulfonate (2g)}

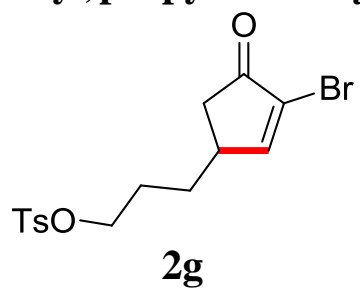

Compound $2 \mathrm{~g}$ was prepared in $72 \%$ yield $(80.5 \mathrm{mg})$ using $1 \mathbf{g}(0.3 \mathrm{mmol})$ as the substrate according to the general procedure E. ${ }^{1} \mathrm{H}$ NMR $(500 \mathrm{MHz}$, Chloroform- $d$ ) $\delta 7.81-7.75$ $(\mathrm{m}, 2 \mathrm{H}), 7.63(\mathrm{~d}, J=2.8 \mathrm{~Hz}, 1 \mathrm{H}), 7.36(\mathrm{~d}, J=8.0 \mathrm{~Hz}, 2 \mathrm{H}), 4.06(\mathrm{t}, J=6.0 \mathrm{~Hz}, 2 \mathrm{H}), 2.94-$ $2.85(\mathrm{~m}, 1 \mathrm{H}), 2.67(\mathrm{dd}, J=18.9,6.4 \mathrm{~Hz}, 1 \mathrm{H}), 2.46(\mathrm{~s}, 3 \mathrm{H}), 2.07(\mathrm{dd}, J=18.9,2.0 \mathrm{~Hz}, 1 \mathrm{H})$, $1.80-1.61(\mathrm{~m}, 3 \mathrm{H}), 1.50(\mathrm{dtd}, J=13.2,8.4,6.1 \mathrm{~Hz}, 1 \mathrm{H}) ;{ }^{13} \mathrm{C} \mathrm{NMR}\left(126 \mathrm{MHz}, \mathrm{CDCl}_{3}\right) \delta$ $200.44,164.21,145.00,132.97,129.92,127.85,126.16,69.62,39.73,39.03,30.47,26.63$, 21.65; IR (neat): 3037, 2922, 2853, 1732, 1581, 1461, 1175.

\section{2-bromo-4-(3-bromopropyl)cyclopent-2-enone (2h)}

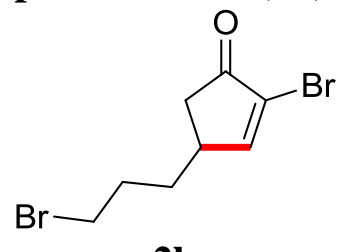

2h

Compound $\mathbf{2 h}$ was prepared in $70 \%$ yield $(59.2 \mathrm{mg})$ using $\mathbf{1 h}(0.3 \mathrm{mmol})$ as the substrate according to the general procedure E. ${ }^{1} \mathrm{H}$ NMR $(500 \mathrm{MHz}$, Chloroform- $d) \delta 7.70(\mathrm{~d}, J=$ $2.7 \mathrm{~Hz}, 1 \mathrm{H}), 3.43(\mathrm{t}, J=6.5 \mathrm{~Hz}, 2 \mathrm{H}), 2.98-2.92(\mathrm{~m}, 1 \mathrm{H}), 2.72(\mathrm{dd}, J=18.9,6.4 \mathrm{~Hz}, 1 \mathrm{H})$, $2.17(\mathrm{dd}, J=18.9,2.0 \mathrm{~Hz}, 1 \mathrm{H}), 1.95(\mathrm{dqd}, J=9.6,6.3,3.5 \mathrm{~Hz}, 2 \mathrm{H}), 1.78$ (ddt, $J=12.5$, 9.7, $6.3 \mathrm{~Hz}, 1 \mathrm{H}), 1.68-1.60(\mathrm{~m}, 1 \mathrm{H}) ;{ }^{13} \mathrm{C} \mathrm{NMR}\left(126 \mathrm{MHz}, \mathrm{CDCl}_{3}\right) \delta 200.57,164.42$, 126.13, 39.73, 39.17, 33.02, 32.74, 30.24; IR (neat): 3065, 2961, 2924, 2858, 1717, 1642, 
1587, 1285, 926; $\mathrm{ESI}^{+}$calculated for $\left[\mathrm{C}_{8} \mathrm{H}_{10} \mathrm{NaBr}_{2} \mathrm{O}+\mathrm{MeOH}\right]^{+}:$334.93, 336.92, 338.92, found $334.85,336.84,338.85$.

\section{2-bromo-4-butyl-5-methylcyclopent-2-enone (2i)}

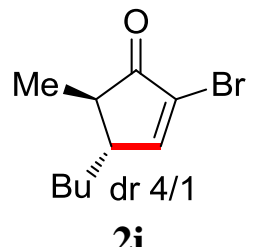

Compound $2 \mathbf{i}$ was prepared in $84 \%$ yield $(58.2 \mathrm{mg})$ using $\mathbf{1 i}(0.3 \mathrm{mmol})$ as the substrate according to the general procedure C. ${ }^{1} \mathrm{H}$ NMR for the major diastereomer $(500 \mathrm{MHz}$, Chloroform- $d$ ) $\delta 7.66(\mathrm{~d}, J=2.7 \mathrm{~Hz}, 1 \mathrm{H}), 2.47-2.40(\mathrm{~m}, 1 \mathrm{H}), 2.09(\mathrm{qd}, J=7.5,2.2 \mathrm{~Hz}$, $1 \mathrm{H}), 1.67-1.30(\mathrm{~m}, 5 \mathrm{H}), 1.23(\mathrm{~d}, J=7.5 \mathrm{~Hz}, 3 \mathrm{H}), 0.92(\mathrm{t}, J=7.0 \mathrm{~Hz}, 3 \mathrm{H})$; selected ${ }^{1} \mathrm{H}$ NMR for the minor diastereomer $(500 \mathrm{MHz}$, Chloroform- $d) \delta 7.77(\mathrm{~d}, J=3.0 \mathrm{~Hz}, 1 \mathrm{H})$, 2.93 (dtd, $J=9.1,6.0,2.9 \mathrm{~Hz}, 1 \mathrm{H}), 2.63$ (dt, $J=14.4,7.5 \mathrm{~Hz}, 1 \mathrm{H}), 1.15$ (d, $J=7.6 \mathrm{~Hz}$, $3 \mathrm{H}) ;{ }^{13} \mathrm{C} \mathrm{NMR}\left(126 \mathrm{MHz}, \mathrm{CDCl}_{3}\right) \delta 204.16,203.93,164.51,163.92,124.65,124.34,49.34$, 45.59, 44.20, 42.64, 33.62, 30.03, 29.85, 29.50, 22.75, 22.67, 15.66, 13.92, 13.89, 11.45; IR (neat): 3063, 2960, 2931, 2872, 1719, 1587, 1456, 1276, 876; $\mathrm{ESI}^{+}$calculated for $\left[\mathrm{C}_{10} \mathrm{H}_{15} \mathrm{NaOBr}\right]^{+}:$253.02, 255.02, found 252.96, 254.96.

\section{2-bromo-4-isopropyl-5-methylcyclopent-2-enone (2j)}

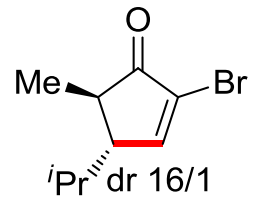

2j

Compound $\mathbf{2} \mathbf{j}$ was prepared in $66 \%$ yield $(43.0 \mathrm{mg})$ using $\mathbf{1} \mathbf{j}(0.3 \mathrm{mmol})$ as the substrate according to the general procedure $\mathbf{C} .{ }^{1} \mathrm{H}$ NMR $(500 \mathrm{MHz}$, Chloroform- $d) \delta 7.70(\mathrm{~d}, J=$ $2.6 \mathrm{~Hz}, 1 \mathrm{H}), 2.31(\mathrm{dt}, J=6.5,2.4 \mathrm{~Hz}, 1 \mathrm{H}), 2.20(\mathrm{qd}, J=7.4,2.2 \mathrm{~Hz}, 1 \mathrm{H}), 1.87-1.78(\mathrm{~m}$, $1 \mathrm{H}), 1.24(\mathrm{~d}, J=7.4 \mathrm{~Hz}, 3 \mathrm{H}), 0.99(\mathrm{~d}, J=6.7 \mathrm{~Hz}, 6 \mathrm{H}) ;{ }^{13} \mathrm{C} \mathrm{NMR}\left(126 \mathrm{MHz}, \mathrm{CDCl}_{3}\right) \delta$ 204.08, 163.02, 124.62, 56.02, 42.68, 31.41, 20.12, 19.96, 16.50; IR (neat): 3068, 2963, 2930, 1718, 1587, 1465, 1374, 875; $\mathrm{ESI}^{+}$calculated for $\left[\mathrm{C} 9 \mathrm{H}_{13} \mathrm{NaOBr}\right]^{+}: 239.00,241.00$, found $238.95,248.95$.
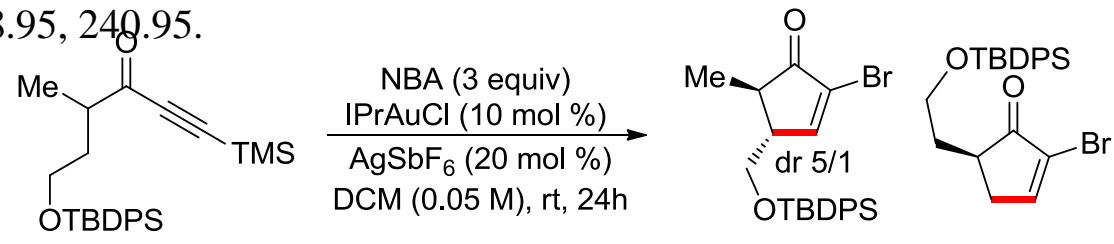

$78 \% \mathbf{2 k} / 2 \mathbf{k}^{\prime}=6 / 1$

Compound $\mathbf{2 k} / \mathbf{2} \mathbf{k}^{\prime}$ was prepared in $78 \%$ total yield $(103.8 \mathrm{mg})$ using $\mathbf{1 k}(0.3 \mathrm{mmol})$ as the substrate according to the general procedure $\mathbf{D}$.

2-bromo-4-(((tert-butyldiphenylsilyl)oxy)methyl)-5-methylcyclopent-2-enone (2k) 


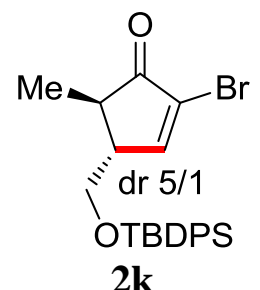

${ }^{1} \mathrm{H}$ NMR (600 MHz, Chloroform- $d$ ) for the major diastereomer $\delta 7.65-7.60(\mathrm{~m}, 4 \mathrm{H}), 7.55$ $(\mathrm{d}, J=2.7 \mathrm{~Hz}, 1 \mathrm{H}), 7.45(\mathrm{t}, J=7.4 \mathrm{~Hz}, 2 \mathrm{H}), 7.40(\mathrm{t}, J=7.4 \mathrm{~Hz}, 4 \mathrm{H}), 3.76$ (qd, $J=10.2$, $5.7 \mathrm{~Hz}, 2 \mathrm{H}), 2.62(\mathrm{tt}, J=5.5,2.6 \mathrm{~Hz}, 1 \mathrm{H}), 2.31(\mathrm{qd}, J=7.5,2.4 \mathrm{~Hz}, 1 \mathrm{H}), 1.21(\mathrm{~d}, J=7.4$ $\mathrm{Hz}, 3 \mathrm{H}), 1.05(\mathrm{~s}, 9 \mathrm{H})$; ); selected ${ }^{1} \mathrm{H}$ NMR for the minor diastereomer $(500 \mathrm{MHz}$, Chloroform- $d$ ) $\delta 7.49(\mathrm{~d}, J=3.1 \mathrm{~Hz}, 1 \mathrm{H}), 3.77(\mathrm{t}, J=4.6 \mathrm{~Hz}, 2 \mathrm{H}), 3.06(\mathrm{p}, J=4.7 \mathrm{~Hz}, 1 \mathrm{H})$, 2.60 (p, $J=7.3 \mathrm{~Hz}, 1 \mathrm{H}), 1.26(\mathrm{~d}, J=7.2 \mathrm{~Hz}, 3 \mathrm{H}), 1.01(\mathrm{~s}, 9 \mathrm{H}) ;{ }^{13} \mathrm{C}$ NMR $(126 \mathrm{MHz}$, $\left.\mathrm{CDCl}_{3}\right) \delta 203.60,161.16,135.59,135.50,132.99,132.93,129.98,129.93,127.85,127.83$, 125.59, 64.30, 51.85, 41.93, 26.80, 19.19, 15.22; IR (neat): 3070, 3045, 2996, 2963, 2927 , 1770, 1757, 1246, 1113, 1060, 1379; $\mathrm{ESI}^{+}$calculated for $\left[\mathrm{C}_{23} \mathrm{H}_{27} \mathrm{NaBrO}_{2} \mathrm{Si}^{+}\right.$: 467.08 , 465.09 , found $464.99,466.98$.

\section{2-bromo-5-(2-((tert-butyldiphenylsilyl)oxy)ethyl)cyclopent-2-enone (2k')

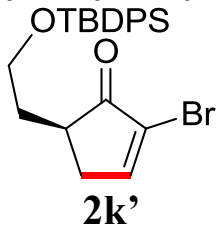

${ }^{1} \mathrm{H}$ NMR $(500 \mathrm{MHz}$, Chloroform- $d$ ) $\delta 7.71(\mathrm{t}, J=3.0 \mathrm{~Hz}, 1 \mathrm{H}), 7.65(\mathrm{dd}, J=7.3,2.6 \mathrm{~Hz}$, $4 \mathrm{H}), 7.41(\mathrm{dq}, J=14.5,7.4 \mathrm{~Hz}, 6 \mathrm{H}), 3.82(\mathrm{dt}, J=11.3,5.8 \mathrm{~Hz}, 1 \mathrm{H}), 3.78-3.70(\mathrm{~m}, 1 \mathrm{H})$, 2.79 (ddd, $J=19.0,6.6,3.2 \mathrm{~Hz}, 1 \mathrm{H}), 2.72-2.65(\mathrm{~m}, 1 \mathrm{H}), 2.41(\mathrm{dt}, J=19.0,2.6 \mathrm{~Hz}, 1 \mathrm{H})$, 2.19 (ddt, $J=13.1,9.4,4.9 \mathrm{~Hz}, 1 \mathrm{H}), 1.59(\mathrm{~m}, 1 \mathrm{H}), 1.04(\mathrm{~s}, 9 \mathrm{H}) ;{ }^{13} \mathrm{C}$ NMR $(126 \mathrm{MHz}$, $\left.\mathrm{CDCl}_{3}\right) \delta 203.75,160.46,135.52,133.49,133.45,129.70,127.71,125.44,61.78,41.17$, 35.01, 33.92, 26.83, 19.15; IR (neat): 3073, 2931, 2858, 1722, 1428, 1111, 823, 742; $\mathrm{ESI}^{+}$ calculated for $\left[\mathrm{C}_{23} \mathrm{H}_{27} \mathrm{NaBrO}_{2} \mathrm{Si}^{+}\right.$: $467.08,465.09$, found $464.99,466.98$.

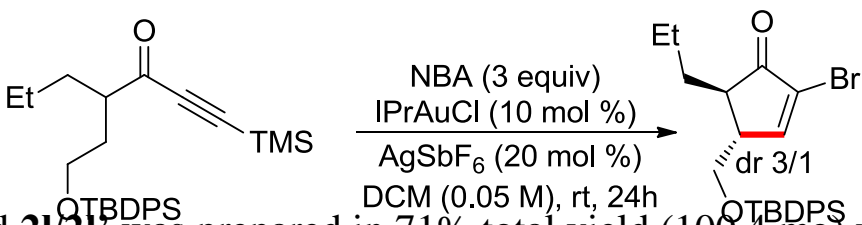

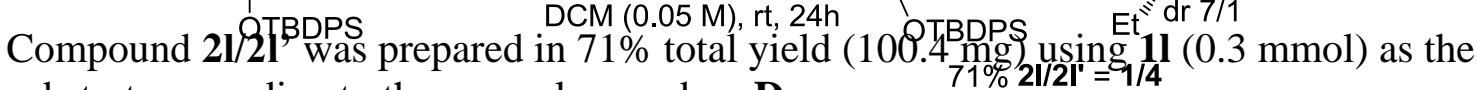
substrate according to the general procedure $\mathbf{D}$.

\section{2-bromo-4-(((tert-butyldiphenylsilyl)oxy)methyl)-5-propylcyclopent-2-enone (2l)}

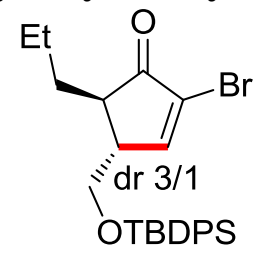

2l 
${ }^{1} \mathrm{H}$ NMR (500 MHz, Chloroform- $d$ ) $\delta 7.62(\mathrm{ddd}, J=7.9,3.6,1.6 \mathrm{~Hz}, 5 \mathrm{H}), 7.48-7.43$ (m, $2 \mathrm{H}), 7.43-7.37(\mathrm{~m}, 4 \mathrm{H}), 3.78(\mathrm{dd}, J=10.1,4.9 \mathrm{~Hz}, 1 \mathrm{H}), 3.66(\mathrm{dd}, J=10.2,6.6 \mathrm{~Hz}, 1 \mathrm{H})$, 2.70 (ddt, $J=7.0,4.9,2.4 \mathrm{~Hz}, 1 \mathrm{H}), 2.22(\mathrm{ddd}, J=8.9,4.8,2.1 \mathrm{~Hz}, 1 \mathrm{H}), 1.74-1.66$ (m, $1 \mathrm{H}), 1.45-1.35(\mathrm{~m}, 1 \mathrm{H}), 1.31-1.22(\mathrm{~m}, 2 \mathrm{H}), 1.05(\mathrm{~s}, 9 \mathrm{H}), 0.87(\mathrm{t}, J=7.3 \mathrm{~Hz}, 3 \mathrm{H}) ;{ }^{13} \mathrm{C}$ NMR $\left(126 \mathrm{MHz}_{2} \mathrm{CDCl}_{3}\right) \delta 203.31,161.91,135.61,135.51,132.98,132.95,129.98$, 129.93, 127.84, 125.77, 64.88, 49.92, 46.48, 33.13, 26.81, 20.17, 19.18, 14.01; IR (neat): 3070, 3053, 2958, 2931, 2858, 1724, 1428, 1112, 741; $\mathrm{ESI}^{+}$calculated for $\left[\mathrm{C}_{25} \mathrm{H}_{31} \mathrm{NaO}_{2} \mathrm{SiBr}\right]^{+}:$493.12, 495.12, found 493.02, 495.02.

\section{2-bromo-5-(2-((tert-butyldiphenylsilyl)oxy)ethyl)-4-ethylcyclopent-2-enone (2l')

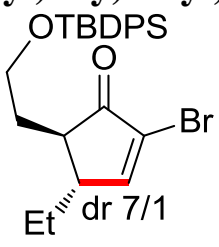 \\ 21 '}

${ }^{1} \mathrm{H}$ NMR (500 MHz, Chloroform- $d$ ) $\delta 7.68(\mathrm{~d}, J=2.7 \mathrm{~Hz}, 1 \mathrm{H}), 7.67-7.64(\mathrm{~m}, 4 \mathrm{H}), 7.46$ $-7.37(\mathrm{~m}, 6 \mathrm{H}), 3.88-3.80(\mathrm{~m}, 1 \mathrm{H}), 3.75(\mathrm{ddd}, J=10.5,7.2,5.3 \mathrm{~Hz}, 1 \mathrm{H}), 2.60(\mathrm{tt}, J=6.7$, $2.4 \mathrm{~Hz}, 1 \mathrm{H}), 2.36$ (ddd, $J=7.9,5.4,2.1 \mathrm{~Hz}, 1 \mathrm{H}), 2.10-2.01(\mathrm{~m}, 1 \mathrm{H}), 1.77-1.60(\mathrm{~m}, 1 \mathrm{H})$, $1.55(\mathrm{dq}, J=14.2,7.4 \mathrm{~Hz}, 1 \mathrm{H}), 1.04(\mathrm{~s}, 9 \mathrm{H}), 0.98(\mathrm{t}, J=7.5 \mathrm{~Hz}, 3 \mathrm{H}) ;{ }^{13} \mathrm{C} \mathrm{NMR}(126 \mathrm{MHz}$, $\left.\mathrm{CDCl}_{3}\right) \delta 203.52,163.84,135.54,135.53,133.52,133.45,129.67,127.68,124.67,61.39$, 48.47, 46.97, 33.73, 26.90, 26.82, 19.13, 11.31; IR (neat): 3071, 3050, 2960, 2931, 2858, 1722, 1589, 1472, 1428, 1111, 702; $\mathrm{ESI}^{+}$calculated for $\left[\mathrm{C}_{25} \mathrm{H}_{31} \mathrm{NaO}_{2} \mathrm{SiBr}\right]^{+}: 493.12$, 495.12, found 493.02, 495.02 .

(E)-methyl 4-((1R,5R)-3-bromo-5-ethyl-2-oxocyclopent-3-en-1-yl)but-2-enoate (2m)<smiles>CCC1C=C(Br)C(=O)[C@@H]1C/C=C/C(=O)O</smiles>

$2 \mathbf{m}$

Compound $2 \mathrm{~m}$ was prepared in $79 \%$ yield $(56.0 \mathrm{mg})$ using $1 \mathrm{~m}(0.3 \mathrm{mmol})$ as the substrate according to the general procedure C. ${ }^{1} \mathrm{H}$ NMR $(500 \mathrm{MHz}$, Chloroform- $d$ ) for the major diastereomer $\delta 7.70(\mathrm{~d}, J=2.7 \mathrm{~Hz}, 1 \mathrm{H}), 6.85(\mathrm{dt}, J=15.3,7.5 \mathrm{~Hz}, 1 \mathrm{H}), 5.90(\mathrm{dt}, J=15.5$, $1.5 \mathrm{~Hz}, 1 \mathrm{H}), 2.74-2.66(\mathrm{~m}, 1 \mathrm{H}), 2.53(\mathrm{tt}, J=6.9,2.5 \mathrm{~Hz}, 1 \mathrm{H}), 2.43(\mathrm{dtd}, J=14.4,8.1,1.4$ $\mathrm{Hz}, 1 \mathrm{H}), 2.26$ (ddd, $J=8.3,4.8,2.2 \mathrm{~Hz}, 1 \mathrm{H}), 1.65-1.52(\mathrm{~m}, 2 \mathrm{H}), 0.99$ (t, $J=7.4 \mathrm{~Hz}, 3 \mathrm{H})$; selected ${ }^{1} \mathrm{H}$ NMR for the minor diastereomer $(500 \mathrm{MHz}$, Chloroform- $d$ ) $\delta 7.86(\mathrm{~d}, J=3.2$ $\mathrm{Hz}, 1 \mathrm{H}), 7.05-6.99(\mathrm{~m}, 1 \mathrm{H}), 3.01-2.96(\mathrm{~m}, 1 \mathrm{H}), 2.81$ (dddd, $J=16.4,6.0,4.3,2.0 \mathrm{~Hz}$, $1 \mathrm{H}), 1.84-1.75(\mathrm{~m}, 1 \mathrm{H}) ;{ }^{13} \mathrm{C} \mathrm{NMR}\left(126 \mathrm{MHz}, \mathrm{CDCl}_{3}\right) \delta 201.87,166.32,164.07,144.64$, 124.58, 123.78, 51.58, 48.77, 47.61, 33.38, 26.84, 11.46; IR (neat): 3063, 2963, 1722, 1659, 1587, 1436, 1276, 1170; $\mathrm{ESI}^{+}$calculated for $\left[\mathrm{C}_{12} \mathrm{H}_{15} \mathrm{NaO}_{3} \mathrm{Br}\right]^{+}$: 309.01, 311.01, found $308.94,310.93$.

\section{2-bromo-5-ethyl-4-isopropylcyclopent-2-enone (2n) 2-bromo-5-isobutyl-4- methylcyclopent-2-enone (2n')}




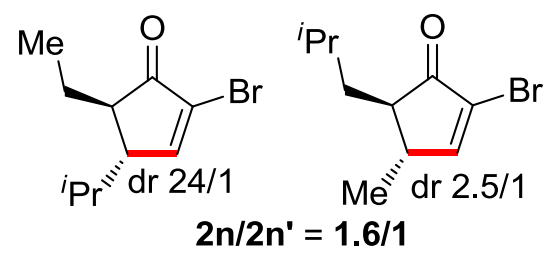

Compound $\mathbf{2 n} / \mathbf{2 n}$ ' was prepared in $82 \%$ total yield $(56.9 \mathrm{mg})$ using $\mathbf{1 n}(0.3 \mathrm{mmol})$ as the substrate according to the general procedure C. Selected ${ }^{1} \mathrm{H}$ NMR for the major diastereomer of 2n' (500 MHz, Chloroform- $d$ ) ${ }^{1} \mathrm{H}$ NMR (500 MHz, Chloroform- $d$ ) $\delta 7.59$ (s, 1H), $2.61(\mathrm{~m}, 1 \mathrm{H}), 2.08(\mathrm{~m}, 1 \mathrm{H}), 1.24(\mathrm{~d}, J=7.2 \mathrm{~Hz}, 3 \mathrm{H}), 0.98(\mathrm{~d}, J=6.8 \mathrm{~Hz}, 6 \mathrm{H})$; Selected ${ }^{1} \mathrm{H}$ NMR for the minor diastereomer of $2 \mathbf{n}$ ' $(500 \mathrm{MHz}$, Chloroform- $d) \delta 3.08(\mathrm{~m}$, $1 \mathrm{H}), 2.54(\mathrm{dt}, J=10.5,5.8 \mathrm{~Hz}, 1 \mathrm{H}), 1.08(\mathrm{~d}, J=7.2 \mathrm{~Hz}, 3 \mathrm{H})$; Selected ${ }^{1} \mathrm{H}$ NMR for the major diastereomer of $2 \mathrm{n}(500 \mathrm{MHz}$, Chloroform- $d$ ) $\delta 7.70(\mathrm{~s}, 1 \mathrm{H}), 2.44(\mathrm{~d}, J=5.8 \mathrm{~Hz}$, $1 \mathrm{H}), 2.16(\mathrm{t}, J=6.4 \mathrm{~Hz}, 1 \mathrm{H}), 1.87-1.80(\mathrm{~m}, 1 \mathrm{H}), 1.66(\mathrm{ddt}, J=21.0,14.2,6.8 \mathrm{~Hz}, 2 \mathrm{H})$; ${ }^{13} \mathrm{C}$ NMR $\left(126 \mathrm{MHz}, \mathrm{CDCl}_{3}\right) \delta 203.99,203.83,203.40,165.39,165.17,163.38,160.56$, $125.01,124.35,124.27,52.92,50.42$, 48.72, 46.24, 42.69, 40.56, 38.77, 34.61, 31.59, $25.96,25.93,24.77,23.39,22.91,21.98,21.62,20.35,19.59,16.15,13.00,10.81$; IR (neat): 3063, 2961, 2931, 2873, 1720, 1588, 1463, 1270, 939; $\mathrm{ESI}^{+}$calculated for $\left[\mathrm{C}_{10} \mathrm{H}_{15} \mathrm{NaOBr}+\mathrm{MeOH}\right]^{+}:$285.05, 287.05, found 284.94, 286.98.

\section{3-bromobicyclo[3.2.0]hept-3-en-2-one (2o)}

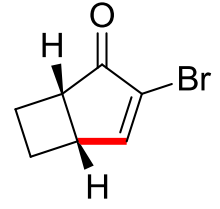

20

Compound 20 was prepared in $73 \%$ yield $(41.0 \mathrm{mg})$ using $10(0.3 \mathrm{mmol})$ as the substrate according to the general procedure C. ${ }^{1} \mathrm{H}$ NMR $(500 \mathrm{MHz}$, Chloroform- $d) \delta 7.82(\mathrm{~d}, J=$ $3.3 \mathrm{~Hz}, 1 \mathrm{H}), 3.47-3.38(\mathrm{~m}, 1 \mathrm{H}), 3.18-3.11(\mathrm{~m}, 1 \mathrm{H}), 2.60-2.44(\mathrm{~m}, 2 \mathrm{H}), 1.90-1.77$ $(\mathrm{m}, 2 \mathrm{H}) ;{ }^{13} \mathrm{C} \mathrm{NMR}\left(126 \mathrm{MHz}, \mathrm{CDCl}_{3}\right) \delta 204.02,163.50,128.62,41.58,39.63,23.28$, 20.04; IR (neat): 3058, 2989, 2948, 1714, 1572, 1385, 1294, 1025, 857; $\mathrm{ESI}^{+}$calculated for $\left[\mathrm{C}_{7} \mathrm{H}_{7} \mathrm{NaOBr}\right]^{+}:$208.96, 210.96, found 208.91, 210.90.

\section{2-bromo-4,5,6,6a-tetrahydropentalen-1(3aH)-one (2p)}

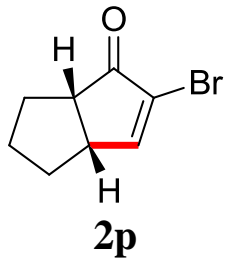

Compound 2p was prepared in $84 \%$ yield $(50.7 \mathrm{mg})$ using $\mathbf{1 p}(0.3 \mathrm{mmol})$ as the substrate according to the general procedure $\mathbf{C} .{ }^{1} \mathrm{H}$ NMR $(500 \mathrm{MHz}$, Chloroform- $d) \delta 7.61(\mathrm{~d}, J=$ $3.1 \mathrm{~Hz}, 1 \mathrm{H}), 3.33$ (ddt, $J=8.8,5.3,2.7 \mathrm{~Hz}, 1 \mathrm{H}), 2.86(\mathrm{dd}, J=10.4,5.7 \mathrm{~Hz}, 1 \mathrm{H}), 1.96$ (dd, $J=13.2,6.2 \mathrm{~Hz}, 1 \mathrm{H}), 1.83-1.62(\mathrm{~m}, 4 \mathrm{H}), 1.30(\mathrm{ddq}, J=17.5,11.2,5.7,5.1 \mathrm{~Hz}, 1 \mathrm{H}) ;{ }^{13} \mathrm{C}$ NMR $\left(126 \mathrm{MHz}, \mathrm{CDCl}_{3}\right) \delta 204.87,164.59,125.65,48.32,45.61,30.35,29.42,23.85$; IR (neat): 3058, 2953, 2866, 1717, 1584, 1277, 935; $\mathrm{ESI}^{+}$calculated for $\left[\mathrm{C}_{8} \mathrm{H} 9 \mathrm{NaOBr}+\mathrm{MeOH}\right]^{+}:$255.00, 257.00, found 254.93, 256.93. 


\section{2-bromo-6a-methyl-4,5,6,6a-tetrahydropentalen-1(3aH)-one (2q)}

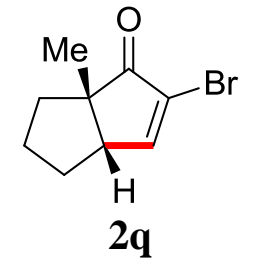

Compound $\mathbf{2 q}$ was prepared in $87 \%$ yield $(56.2 \mathrm{mg})$ using $\mathbf{1 q}(0.3 \mathrm{mmol})$ as the substrate according to the general procedure C. ${ }^{1} \mathrm{H}$ NMR $(500 \mathrm{MHz}$, Chloroform- $d) \delta 7.57(\mathrm{~d}, J=$ $3.0 \mathrm{~Hz}, 1 \mathrm{H}), 2.87(\mathrm{dt}, J=9.5,2.3 \mathrm{~Hz}, 1 \mathrm{H}), 1.97(\mathrm{dd}, J=13.0,6.1 \mathrm{~Hz}, 1 \mathrm{H}), 1.83$ (tdd, $J=$ $12.1,8.9,6.2 \mathrm{~Hz}, 1 \mathrm{H}), 1.70-1.59(\mathrm{~m}, 2 \mathrm{H}), 1.42(\mathrm{td}, J=12.5,6.2 \mathrm{~Hz}, 1 \mathrm{H}), 1.29$ (ddd, $J=$ 19.9, 12.9, 7.0 Hz, 1H), $1.24(\mathrm{~s}, 3 \mathrm{H}) ;{ }^{13} \mathrm{C} \mathrm{NMR}\left(126 \mathrm{MHz}, \mathrm{CDCl}_{3}\right) \delta 206.81,163.35$, 124.39, 53.44, 53.39, 38.32, 29.29, 24.58, 22.29; IR (neat): 3060, 2956, 2867, 1718, 1586, $1448,910,815$ ESI $^{+}$calculated for $\left[\mathrm{C}_{9} \mathrm{H}_{11} \mathrm{NaOBr}\right]^{+}: 236.99,238.99$, found 236.93, 238.93.

\section{2-bromo-3a,4,5,6,7,7a-hexahydro-1H-inden-1-one (2r)}

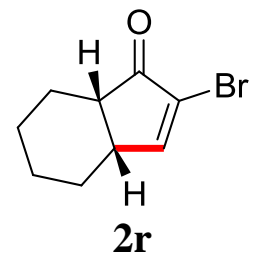

Compound $2 \mathbf{r}$ was prepared in $74 \%$ yield $(47.7 \mathrm{mg})$ using $\mathbf{1 r}(0.3 \mathrm{mmol})$ as the substrate according to the general procedure C. ${ }^{1} \mathrm{H}$ NMR $(600 \mathrm{MHz}$, Chloroform- $d) \delta 7.72(\mathrm{~d}, J=$ $3.2 \mathrm{~Hz}, 1 \mathrm{H}), 3.02-2.95(\mathrm{~m}, 1 \mathrm{H}), 2.56(\mathrm{q}, J=6.3 \mathrm{~Hz}, 1 \mathrm{H}), 2.02-1.91(\mathrm{~m}, 2 \mathrm{H}), 1.75(\mathrm{tt}, J$ $=14.0,6.4 \mathrm{~Hz}, 1 \mathrm{H}), 1.53(\mathrm{ddt}, J=17.7,11.4,6.0 \mathrm{~Hz}, 2 \mathrm{H}), 1.39(\mathrm{ddq}, J=15.2,7.4,4.5,3.6$ $\mathrm{Hz}, 1 \mathrm{H}), 1.28(\mathrm{ddq}, J=19.9,10.4,5.0 \mathrm{~Hz}, 1 \mathrm{H}), 1.23-1.15(\mathrm{~m}, 1 \mathrm{H}) ;{ }^{13} \mathrm{C} \mathrm{NMR}(126 \mathrm{MHz}$, $\left.\mathrm{CDCl}_{3}\right) \delta 203.42,164.81,124.83,44.31,40.40,28.05,22.89,21.22,21.03$; IR (neat): 3063, $2937,2858,1725,1581,1448,1272 ; \mathrm{ESI}^{+}$calculated for $\left[\mathrm{C}_{9} \mathrm{H}_{11} \mathrm{NaOBr}+\mathrm{MeOH}\right]^{+}: 269.02$, 271.01 , found $268.94,270.95$.

\section{2-bromo-7a-methyl-3a,4,5,6,7,7a-hexahydro-1H-inden-1-one (2s)}

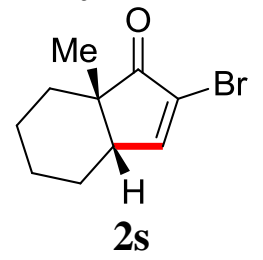

Compound $2 \mathbf{s}$ was prepared in $90 \%$ yield $(61.8 \mathrm{mg})$ using $1 \mathbf{s}(0.3 \mathrm{mmol})$ as the substrate according to the general procedure C. ${ }^{1} \mathrm{H}$ NMR $(500 \mathrm{MHz}$, Chloroform- $d) \delta 7.60(\mathrm{~d}, J=$ $2.7 \mathrm{~Hz}, 1 \mathrm{H}), 2.62(\mathrm{td}, J=5.7,2.8 \mathrm{~Hz}, 1 \mathrm{H}), 1.85(\mathrm{dddd}, J=13.9,10.2,6.3,4.2 \mathrm{~Hz}, 1 \mathrm{H})$, $1.68-1.41(\mathrm{~m}, 5 \mathrm{H}), 1.39-1.31(\mathrm{~m}, 2 \mathrm{H}), 1.17(\mathrm{~s}, 3 \mathrm{H}) ;{ }^{13} \mathrm{C} \mathrm{NMR}\left(126 \mathrm{MHz}, \mathrm{CDCl}_{3}\right) \delta$ 206.71, 163.61, 123.89, 48.36, 46.25, 31.05, 25.44, 22.90, 19.64, 19.15; IR (neat): 3068, 2939, 2862, 1727, 1587, 1449, 1379, 1047, 743; $\mathrm{ESI}^{+}$calculated for $\left[\mathrm{C}_{10} \mathrm{H}_{13} \mathrm{KOBr}+\mathrm{MeOH}\right]^{+}:$299.00, 301.00, found 298.96, 300.96.

\section{2-bromo-5,7a-dimethyl-3a,4,5,6,7,7a-hexahydro-1H-inden-1-one (2t)}




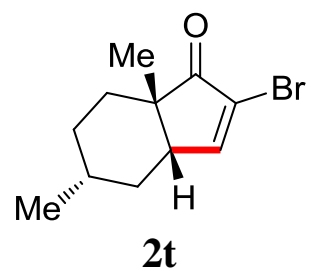

Compound $2 \mathbf{t}$ was prepared in $93 \%$ yield $(67.8 \mathrm{mg})$ using $\mathbf{1 t}(0.3 \mathrm{mmol})$ as the substrate according to the general procedure C. ${ }^{1} \mathrm{H}$ NMR $(500 \mathrm{MHz}$, Chloroform- $d) \delta 7.55(\mathrm{~d}, J=$ $2.5 \mathrm{~Hz}, 1 \mathrm{H}), 2.72(\mathrm{dt}, J=6.4,2.5 \mathrm{~Hz}, 1 \mathrm{H}), 1.80$ (ddd, $J=13.5,4.6,2.5 \mathrm{~Hz}, 1 \mathrm{H}), 1.56-$ $1.34(\mathrm{~m}, 5 \mathrm{H}), 1.19(\mathrm{~s}, 3 \mathrm{H}), 1.09-1.00(\mathrm{~m}, 1 \mathrm{H}), 0.91(\mathrm{~d}, J=6.4 \mathrm{~Hz}, 3 \mathrm{H}) ;{ }^{13} \mathrm{C}$ NMR $(126$ $\left.\mathrm{MHz}, \mathrm{CDCl}_{3}\right) \delta$ 206.61, 163.33, 123.49, 48.32, 46.23, 33.88, 32.17, 28.99, 26.81, 22.44, 20.79; IR (neat): 3068, 2927, 2869, 1723, 1589, 1456, 1378, 1282, 763; ESI ${ }^{+}$calculated for $\left[\mathrm{C}_{11} \mathrm{H}_{15} \mathrm{KOBr}+\mathrm{MeOH}\right]^{+}: 313.02,315.02$, found 312.90, 314.90 .

\section{2-bromo-8a-methyl-4,5,6,7,8,8a-hexahydroazulen-1(3aH)-one (2u)}

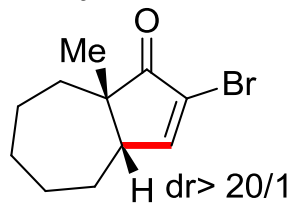

$2 \mathbf{u}$

Compound $\mathbf{2 u}$ was prepared in $90 \%$ yield $(65.6 \mathrm{mg})$ using $\mathbf{1 u}(0.3 \mathrm{mmol})$ as the substrate according to the general procedure C. ${ }^{1} \mathrm{H}$ NMR $(500 \mathrm{MHz}$, Chloroform- $d) \delta 7.60(\mathrm{~d}, J=$ $2.9 \mathrm{~Hz}, 1 \mathrm{H}), 2.70(\mathrm{dt}, J=8.9,3.2 \mathrm{~Hz}, 1 \mathrm{H}), 1.91$ (ddd, $J=14.0,9.6,3.5 \mathrm{~Hz}, 1 \mathrm{H}), 1.72-$ $1.67(\mathrm{~m}, 2 \mathrm{H}), 1.63-1.34(\mathrm{~m}, 7 \mathrm{H}), 1.14(\mathrm{~s}, 3 \mathrm{H}) ;{ }^{13} \mathrm{C} \mathrm{NMR}\left(126 \mathrm{MHz}, \mathrm{CDCl}_{3}\right) \delta 207.49$, 164.96, 123.09, 54.68, 50.17, 35.02, 31.20, 30.91, 27.35, 26.17, 24.67; IR (neat): 3058 , 2925, 2854, 1720, 1592, 1448, 1277, 816; $\mathrm{ESI}^{+}$calculated for $\left[\mathrm{C}_{11} \mathrm{H}_{15} \mathrm{NaOBr}+\mathrm{MeOH}\right]^{+}$: 297.05, 299.04, found 296.98, 298.98 .

\section{2-bromo-5,6,7,8,9,9a-hexahydro-3a,7:5,9-dimethanocyclopenta[8]annulen-3(4H)-one} $(2 \mathrm{v})$

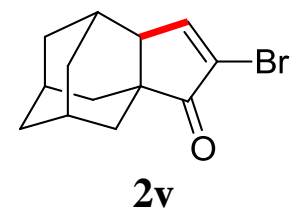

Compound $2 \mathbf{v}$ was prepared in $79 \%$ yield $(63.3 \mathrm{mg})$ using $\mathbf{1 v}(0.3 \mathrm{mmol})$ as the substrate according to the general procedure C. ${ }^{1} \mathrm{H}$ NMR $(500 \mathrm{MHz}$, Chloroform- $d) \delta 7.61(\mathrm{~d}, J=$ $1.6 \mathrm{~Hz}, 1 \mathrm{H}), 2.88(\mathrm{~s}, 1 \mathrm{H}), 2.30(\mathrm{~m}, 1 \mathrm{H}), 2.12(\mathrm{~m}, 2 \mathrm{H}), 1.98(\mathrm{~m}, 1 \mathrm{H}), 1.86(\mathrm{~m}, 2 \mathrm{H}), 1.78$ $(\mathrm{m}, 5 \mathrm{H}), 1.71-1.60(\mathrm{~m}, 2 \mathrm{H}) ;{ }^{13} \mathrm{C} \mathrm{NMR}\left(126 \mathrm{MHz}, \mathrm{CDCl}_{3}\right) \delta 203.95,159.02,124.71$, 52.96, 49.22, 39.18, 38.79, 36.76, 35.19, 32.30, 30.25, 28.27, 27.60; IR (neat): 3053, 2919, 2855, 1716, 1447, 1242, 923; $\mathrm{ESI}^{+}$calculated for $\left[\mathrm{C}_{13} \mathrm{H}_{15} \mathrm{NaOBr}+\mathrm{MeOH}\right]^{+}: 321.05$, 323.04, found 320.97, 322.97 .

\section{2-bromo-6-((tert-butyldiphenylsilyl)oxy)-3a,4,5,6,7,7a-hexahydro-1H-inden-1-one $(2 \mathrm{w})$}




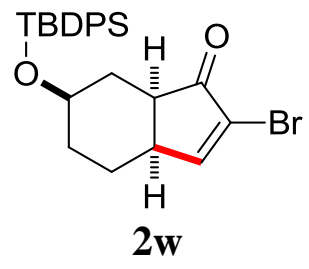

Compound $\mathbf{2 w}$ was prepared in $74 \%$ yield $(34.8 \mathrm{mg})$ using $\mathbf{1 w}(0.1 \mathrm{mmol})$ as the substrate according to the general procedure C. ${ }^{1} \mathrm{H}$ NMR $(500 \mathrm{MHz}$, Chloroform- $d) \delta 7.70(\mathrm{~d}, J=$ $3.2 \mathrm{~Hz}, 1 \mathrm{H}), 7.69-7.66(\mathrm{~m}, 2 \mathrm{H}), 7.64-7.59(\mathrm{~m}, 2 \mathrm{H}), 7.44-7.31(\mathrm{~m}, 6 \mathrm{H}), 3.98$ (ddd, $J=$ $8.9,5.2,3.8 \mathrm{~Hz}, 1 \mathrm{H}), 2.91(\mathrm{dtd}, J=9.7,6.7,3.2 \mathrm{~Hz}, 1 \mathrm{H}), 2.50-2.44(\mathrm{~m}, 1 \mathrm{H}), 2.20(\mathrm{dt}, J$ $=14.2,5.3 \mathrm{~Hz}, 1 \mathrm{H}), 1.77$ (dddd, $J=26.2,11.9,7.5,4.3 \mathrm{~Hz}, 2 \mathrm{H}), 1.70-1.64(\mathrm{~m}, 1 \mathrm{H}), 1.41$ (dq, $J=10.3,4.3 \mathrm{~Hz}, 2 \mathrm{H}), 1.01(\mathrm{~s}, 9 \mathrm{H}) ;{ }^{13} \mathrm{C}$ NMR $\left(126 \mathrm{MHz}, \mathrm{CDCl}_{3}\right) \delta 202.30,163.76$, 135.94, 135.78, 134.27, 133.77, 129.61, 129.54, 127.54, 127.51, 124.42, 66.53, 41.72, 39.07, 31.53, 30.53, 26.90, 23.37, 19.07; IR (neat): 3070, 3050, 2996, 2930, 2856, 1727, 1428, 1108, 1067, 1009; $\mathrm{ESI}^{+}$calculated for $\left[\mathrm{C}_{25} \mathrm{H}_{29} \mathrm{NaO}_{2} \mathrm{SiBr}\right]^{+}$: 493.10, 491.10, found 491.01, 493.01 .

\section{2-bromo-6-((tert-butyldiphenylsilyl)oxy)-3a,4,5,6,7,7a-hexahydro-1H-inden-1-one} $(2 x)$

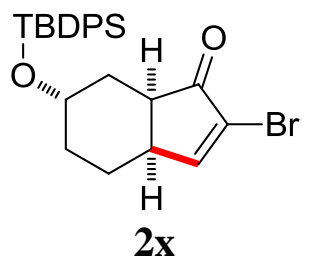

Compound $\mathbf{2 x}$ was prepared in $78 \%$ yield $(36.7 \mathrm{mg})$ using $\mathbf{1 x}(0.1 \mathrm{mmol})$ as the substrate according to the general procedure $\mathbf{C} .{ }^{1} \mathrm{H}$ NMR $(400 \mathrm{MHz}$, Chloroform- $d) \delta 7.64(\mathrm{~m}, 5 \mathrm{H})$, $7.48-7.33(\mathrm{~m}, 6 \mathrm{H}), 3.87(\mathrm{p}, J=5.5 \mathrm{~Hz}, 1 \mathrm{H}), 3.06-2.97(\mathrm{~m}, 1 \mathrm{H}), 2.83(\mathrm{q}, J=6.9 \mathrm{~Hz}$, 1H), $2.08-1.97(\mathrm{~m}, 1 \mathrm{H}), 1.89(\mathrm{t}, J=6.4 \mathrm{~Hz}, 2 \mathrm{H}), 1.60(\mathrm{dd}, J=14.0,5.5 \mathrm{~Hz}, 1 \mathrm{H}), 1.45$ (ddt, $J=14.2,10.4,5.0 \mathrm{~Hz}, 1 \mathrm{H}), 1.06(\mathrm{~m}, 10 \mathrm{H}) ;{ }^{13} \mathrm{C} \mathrm{NMR}\left(126 \mathrm{MHz}, \mathrm{CDCl}_{3}\right) \delta 203.27$, 164.05, 135.69, 135.66, 134.31, 133.92, 129.71, 129.61, 127.66, 127.58, 124.79, 67.22, 42.52, 39.73, 31.50, 31.34, 26.97, 25.56, 19.16; IR (neat): 3073, 3045, 2931, 2857, 1720, 1427, 1110, 822; $\mathrm{ESI}^{+}$calculated for $\left[\mathrm{C}_{25} \mathrm{H}_{29} \mathrm{NaO}_{2} \mathrm{SiBr}\right]^{+}: 493.10,491.10$, found 491.01, 493.01 . 


\begin{tabular}{|ll|}
\hline \multicolumn{1}{|c|}{ Parameter } & \multicolumn{1}{c|}{ Value } \\
1 Title & youliang-5-195-A-SM \\
2 Solvent & CDCl3 \\
3 Temperature & 25.0 \\
4 Relaxation Delay & 10.0000 \\
5 Spectrometer Frequency & 499.86 \\
\hline
\end{tabular}
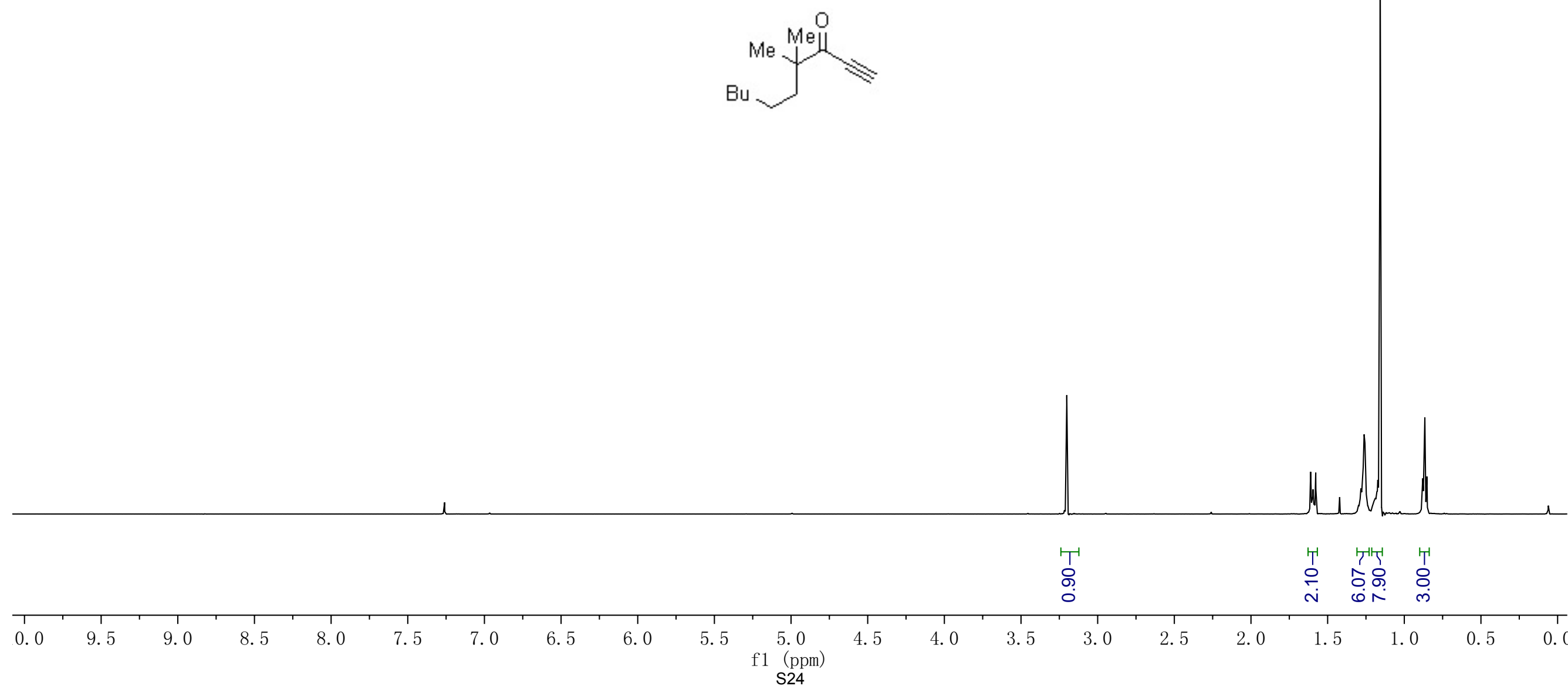


\begin{tabular}{|ll|}
\hline \multicolumn{1}{|c|}{ Parameter } & \multicolumn{1}{c|}{ Value } \\
1 Title & youliang-5-195-A-SM-C13e \\
2 Solvent & $\mathrm{CDCl} 3$ \\
3 Temperature & 25.0 \\
4 Relaxation Delay & 1.0000 \\
5 Spectrometer Frequency & 125.70 \\
\hline
\end{tabular}

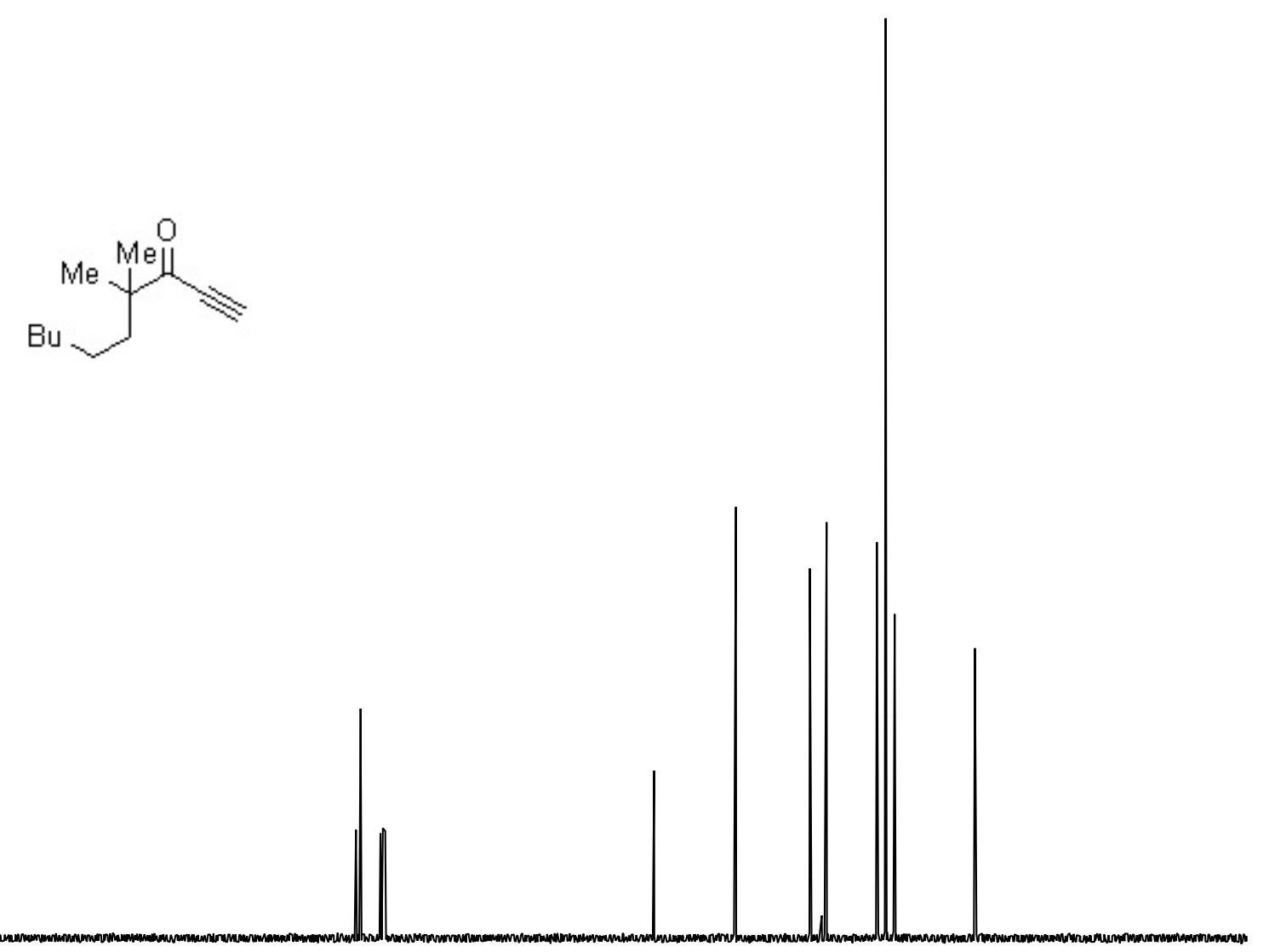

\begin{tabular}{|c|c|c|c|c|c|c|c|c|c|c|c|c|c|c|c|c|c|c|c|c|c|c|c|c|}
\hline 220 & 210 & 200 & 190 & 180 & 170 & 160 & 150 & 140 & 130 & 120 & $\begin{array}{r}110 \\
\mathrm{f} 1\end{array}$ & $\begin{array}{l}100 \\
(\mathrm{ppm}) \\
25\end{array}$ & 90 & 80 & 70 & 6 & & 50 & 40 & 30 & 20 & 10 & 0 & -10 \\
\hline
\end{tabular}




\begin{tabular}{|ll|}
\hline \multicolumn{1}{|c|}{ Parameter } & \multicolumn{1}{c|}{ Value } \\
1 Title & youliang-5-194-A-P \\
2 Solvent & CDCl3 \\
3 Spectrometer Frequency & 499.86 \\
4 Nucleus & $1 \mathrm{H}$ \\
\hline
\end{tabular}
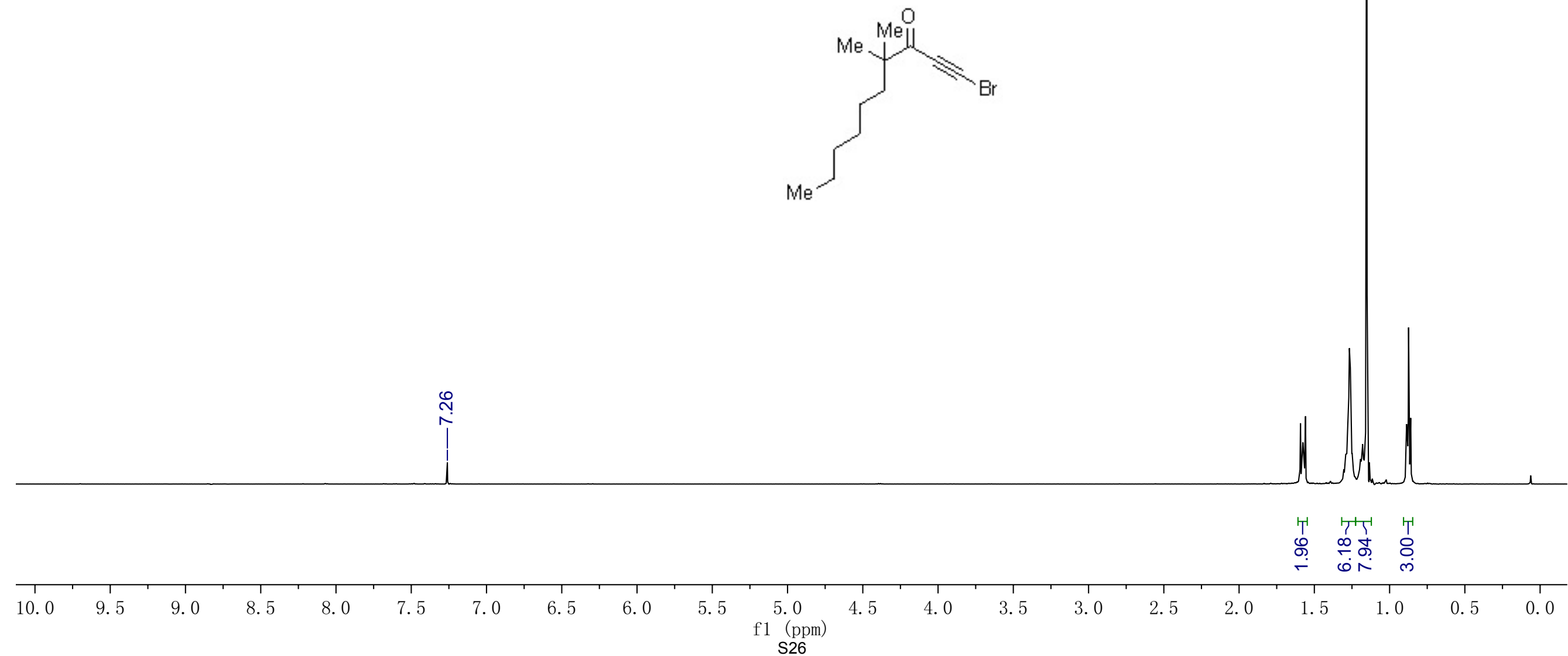
Spectrometer Frequency 125.70
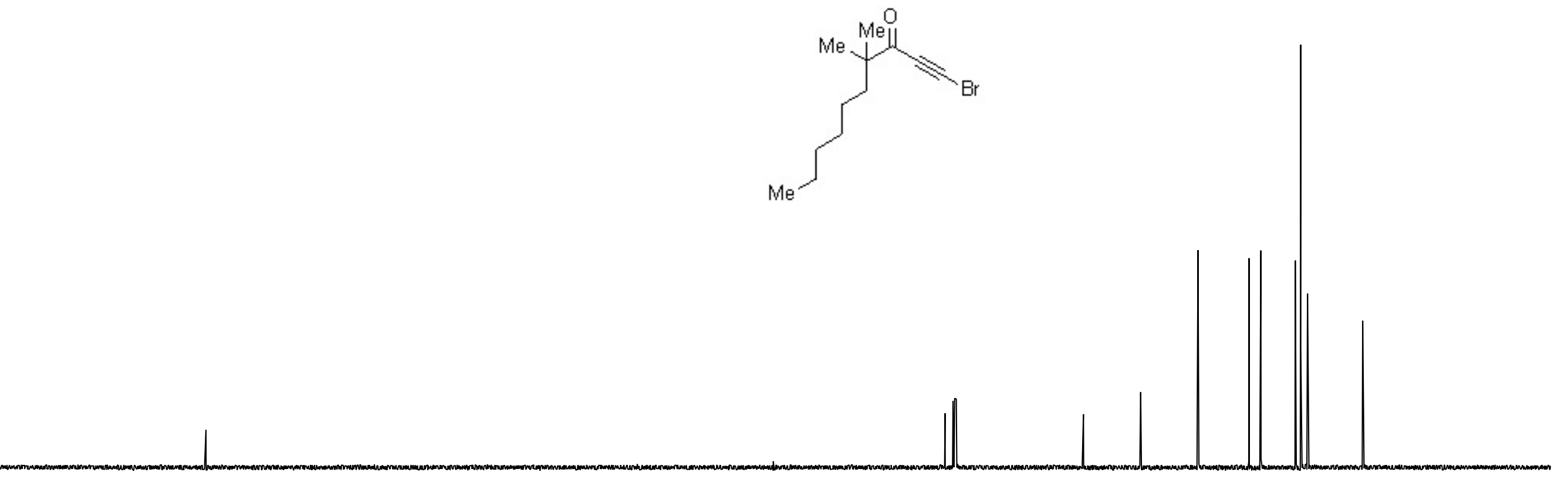

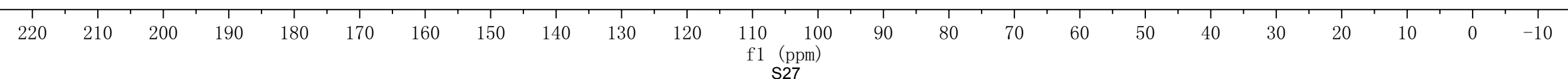




\begin{tabular}{|ll|}
\hline \multicolumn{1}{|c|}{ Parameter } & \multicolumn{1}{c|}{ Value } \\
1 Title & youliang-5-298-A-SM \\
2 Solvent & CDCl3 \\
3 Temperature & 25.0 \\
4 Relaxation Delay & 10.0000 \\
5 Spectrometer Frequency & 499.86 \\
\hline
\end{tabular}

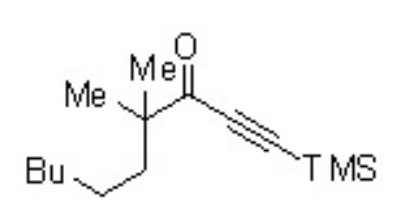




\begin{tabular}{|ll|}
\hline \multicolumn{1}{|c|}{ Parameter } & \multicolumn{1}{c|}{ Value } \\
1 Title & youliang-5-298-A-SM-C13f \\
2 Solvent & $\mathrm{CDCl3}$ \\
3 Temperature & 25.0 \\
4 Relaxation Delay & 1.0000 \\
5 Spectrometer Frequency & 125.70 \\
\hline
\end{tabular}
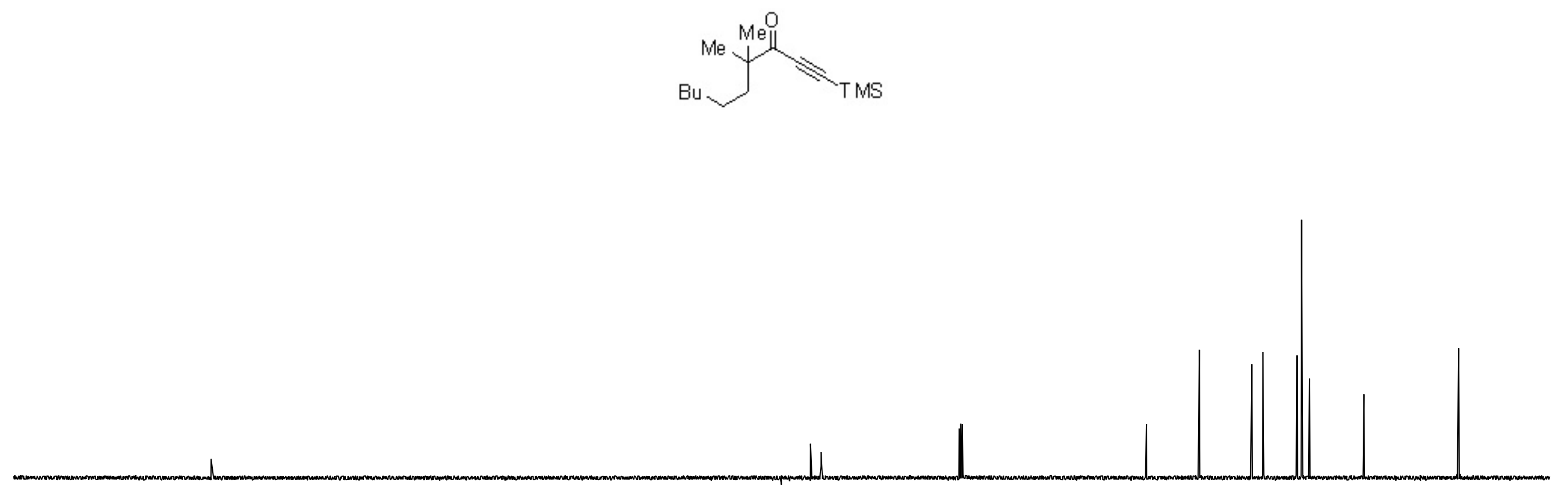

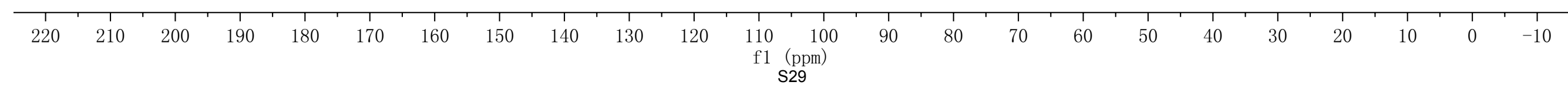




\begin{tabular}{|ll|}
\hline \multicolumn{1}{|c|}{ Parameter } & \multicolumn{1}{c|}{ Value } \\
1 Title & youliang-5-TES-ynone \\
2 Solvent & CDCl3 \\
3 Spectrometer Frequency & 499.86 \\
4 Nucleus & $1 \mathrm{H}$ \\
\hline
\end{tabular}
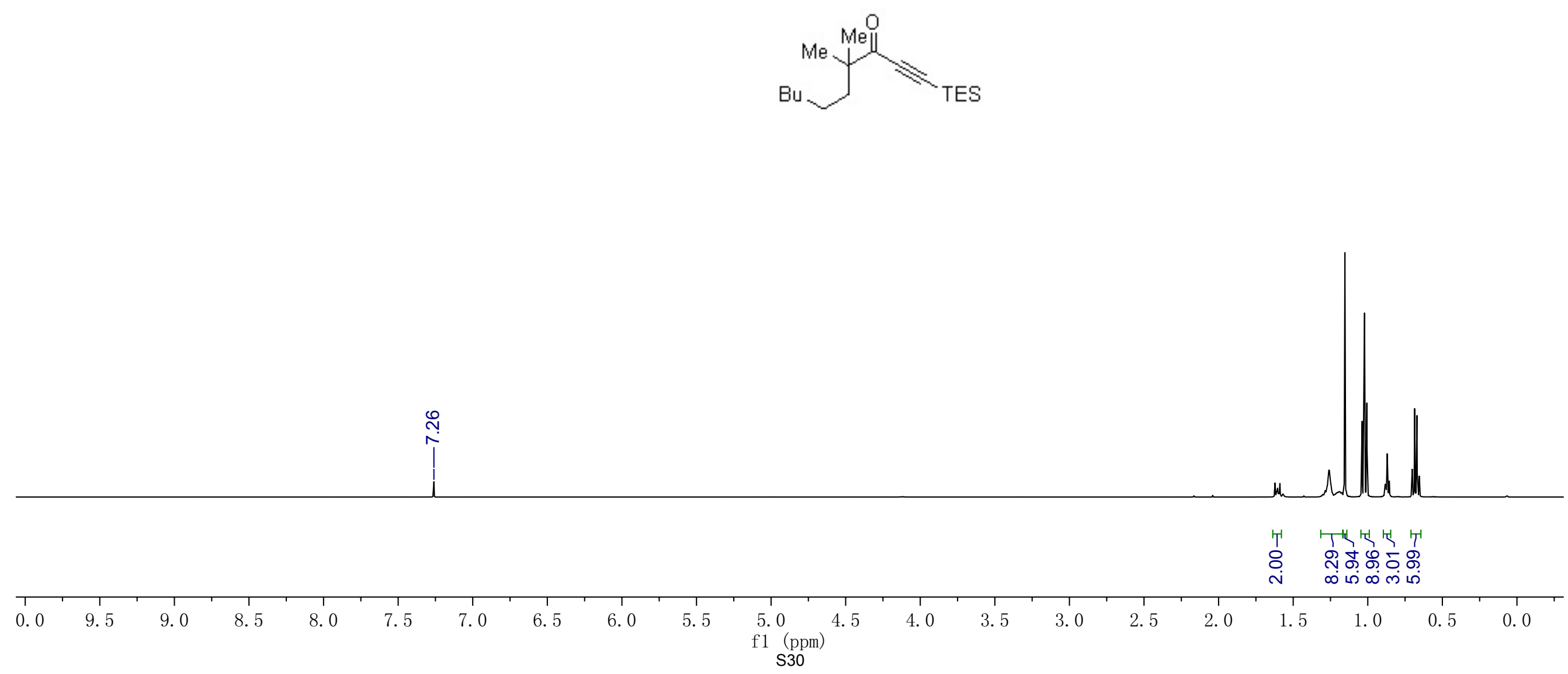


\begin{tabular}{|ll|}
\hline \multicolumn{1}{|c|}{ Parameter } & \multicolumn{1}{c|}{ Value } \\
1 Title & youliang-5-TES-ynone-C13i \\
2 Solvent & $\mathrm{CDCl} 3$ \\
3 Spectrometer Frequency & 125.70 \\
4 Nucleus & $13 \mathrm{C}$ \\
\hline
\end{tabular}

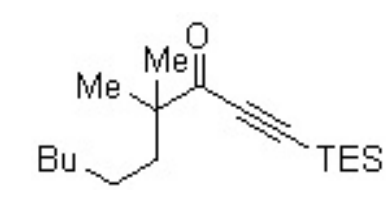

\begin{tabular}{|c|c|c|c|c|c|c|c|c|c|c|c|c|c|c|c|c|c|c|c|c|c|c|c|}
\hline 220 & 210 & 200 & 190 & 180 & 170 & 160 & 150 & 140 & 130 & 120 & $\begin{array}{r}110 \\
\mathrm{f} 1\end{array}$ & $\begin{array}{c}100 \\
(\mathrm{ppm}) \\
331\end{array}$ & 90 & 80 & 70 & 60 & 50 & 40 & 30 & 20 & 10 & 0 & -10 \\
\hline
\end{tabular}




\begin{tabular}{|ll|}
\hline \multicolumn{1}{|c|}{ Parameter } & \multicolumn{1}{c|}{ Value } \\
1 Title & youliang-5-300-B-SM \\
2 Solvent & $\mathrm{CDCl3}$ \\
3 Temperature & 25.0 \\
4 Relaxation Delay & 10.0000 \\
5 Spectrometer Frequency & 499.86 \\
\hline
\end{tabular}
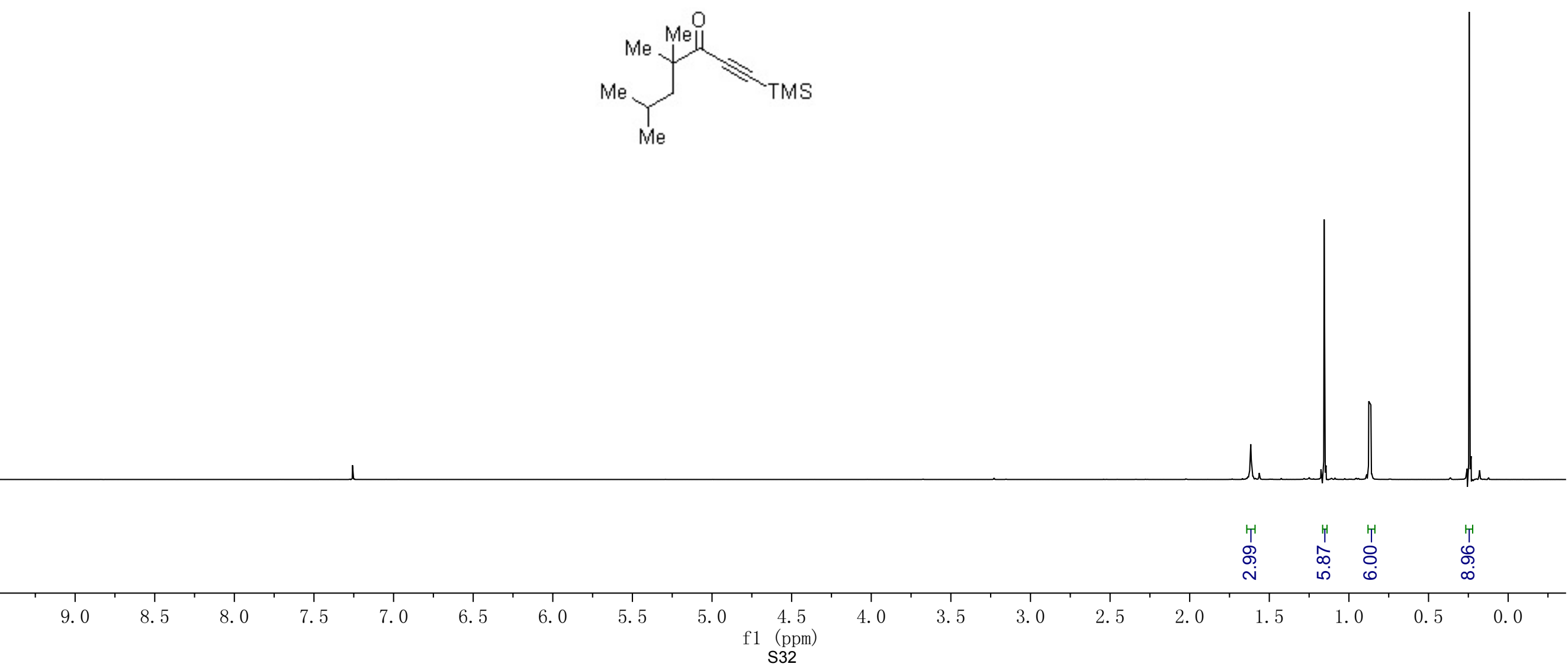


\begin{tabular}{|ll|}
\hline \multicolumn{1}{|c|}{ Parameter } & \multicolumn{1}{c|}{ Value } \\
1 Title & youliang-5-300-B-SM-C13g \\
2 Solvent & CDCl3 \\
3 Temperature & 25.0 \\
4 Relaxation Delay & 1.0000 \\
5 Spectrometer Frequency & 125.70 \\
\hline
\end{tabular}

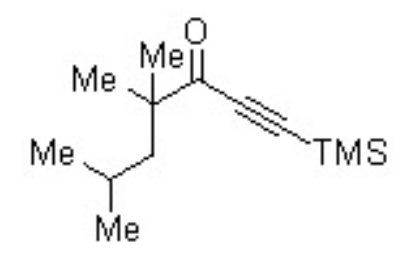

numurnem

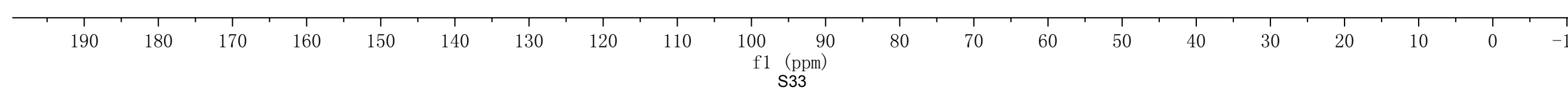




\begin{tabular}{|ll|}
\hline \multicolumn{1}{|c|}{ Parameter } & \multicolumn{1}{c|}{ Value } \\
1 Title & youliang-5-279-A-SM \\
2 Solvent & $\mathrm{CDCl3}$ \\
3 Spectrometer Frequency & 499.86 \\
4 Nucleus & $1 \mathrm{H}$ \\
\hline
\end{tabular}
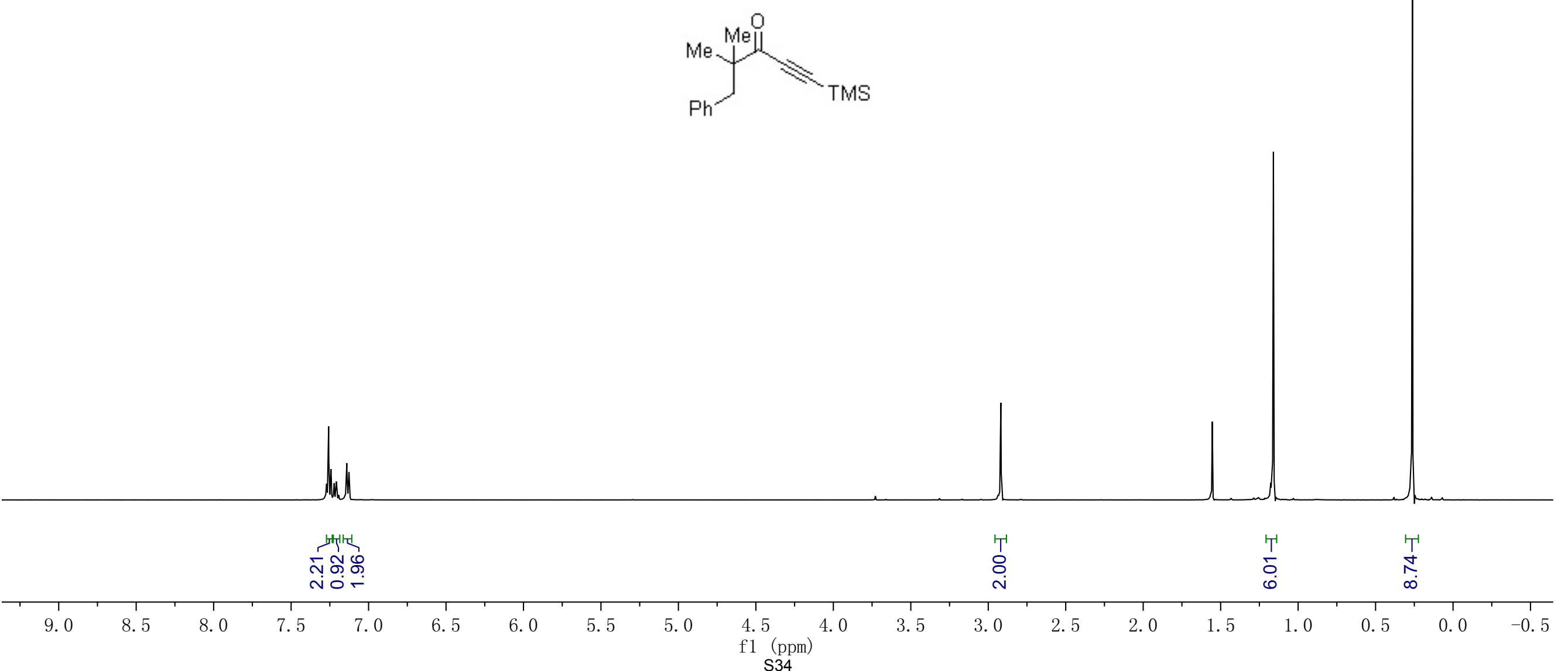


\begin{tabular}{|ll|}
\hline \multicolumn{1}{|c|}{ Parameter } & \multicolumn{1}{c|}{ Value } \\
1 Title & youliang-5-279-A-SM-C13f \\
2 Solvent & $\mathrm{CDCl3}$ \\
3 Spectrometer Frequency & 125.70 \\
4 Nucleus & $13 \mathrm{C}$ \\
\hline
\end{tabular}
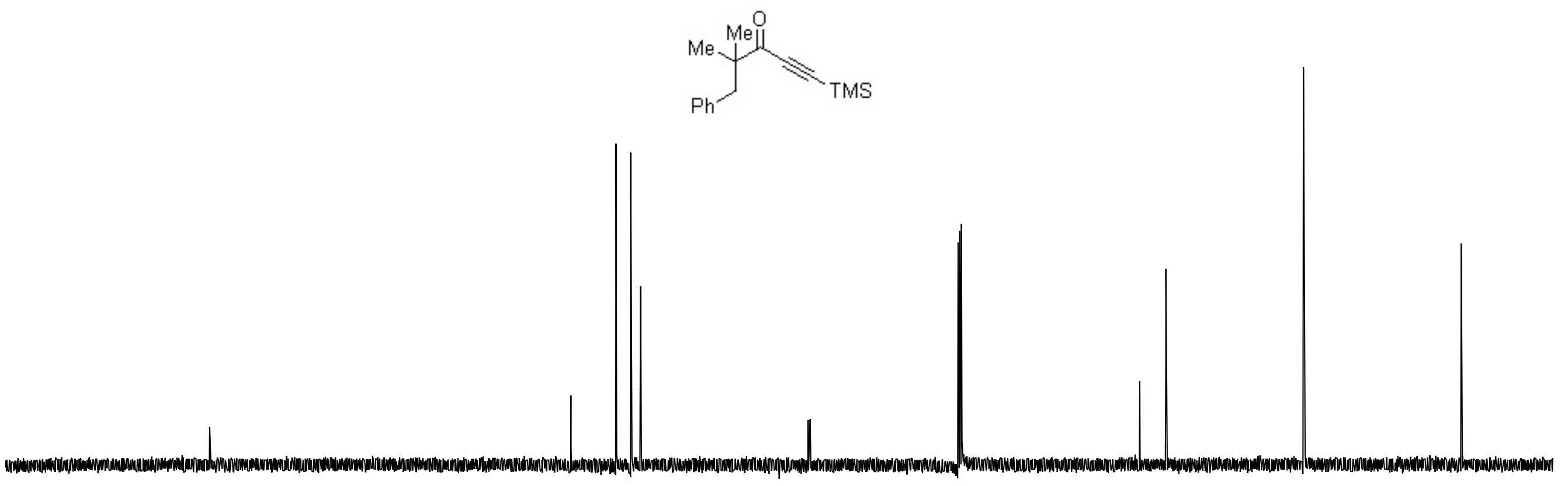

\begin{tabular}{|c|c|c|c|c|c|c|c|c|c|c|c|c|c|c|c|c|c|c|c|c|c|c|c|}
\hline $\begin{array}{c}1 \\
220\end{array}$ & 210 & 200 & $\begin{array}{c}1 \\
190\end{array}$ & 180 & $\begin{array}{c}1 \\
170\end{array}$ & 160 & $\begin{array}{c}1 \\
150\end{array}$ & 140 & 130 & 120 & $\begin{array}{r}110 \\
\mathrm{f} 1 \\
\mathrm{~s}\end{array}$ & $\begin{array}{c}100 \\
(\mathrm{ppm}) \\
35\end{array}$ & $\begin{array}{l}1 \\
90\end{array}$ & $\begin{array}{l}1 \\
80\end{array}$ & $\begin{array}{c}1 \\
70\end{array}$ & $\begin{array}{c}1 \\
60\end{array}$ & $\begin{array}{c}1 \\
50\end{array}$ & 10 & $\begin{array}{l}1 \\
30\end{array}$ & $\begin{array}{c}1 \\
20\end{array}$ & 10 & $\begin{array}{l}1 \\
0\end{array}$ & $\begin{array}{c}1 \\
-10\end{array}$ \\
\hline
\end{tabular}




\begin{tabular}{|ll|}
\hline \multicolumn{1}{|c|}{ Parameter } & \multicolumn{1}{c|}{ Value } \\
1 Title & youliang-5-302-C-P1 \\
2 Solvent & $\mathrm{CDCl3}$ \\
3 Temperature & 25.0 \\
4 Relaxation Delay & 4.8000 \\
5 Spectrometer Frequency & 499.86 \\
\hline
\end{tabular}
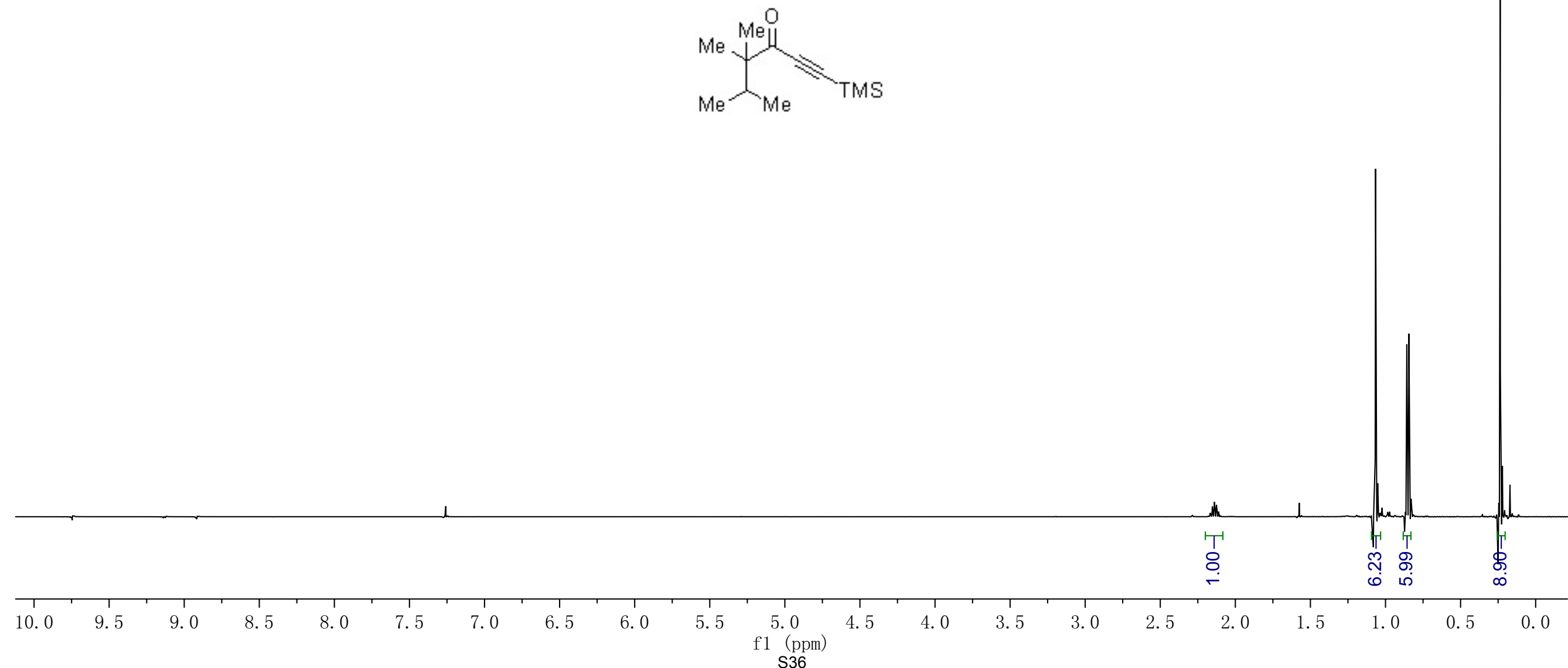


\begin{tabular}{|ll|}
\hline \multicolumn{1}{|c|}{ Parameter } & \multicolumn{1}{c|}{ Value } \\
1 Title & youliang-5-302-C-P1-C13h \\
2 Solvent & CDCl3 \\
3 Temperature & 25.0 \\
4 Relaxation Delay & 1.0000 \\
5 Spectrometer Frequency & 125.70 \\
\hline
\end{tabular}
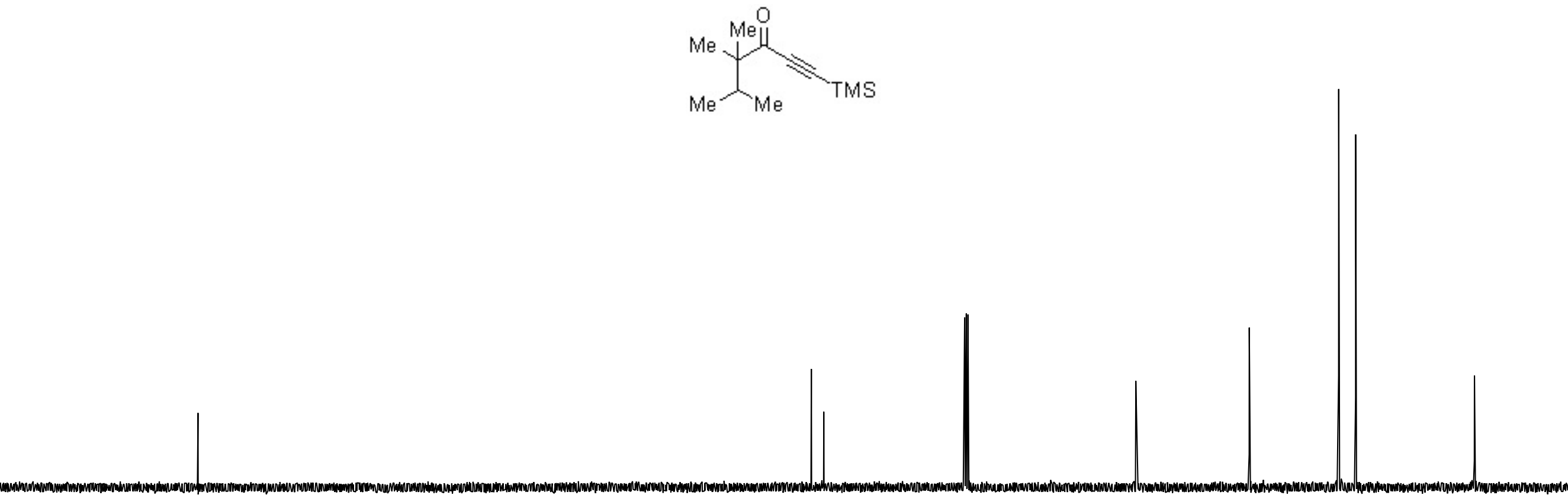

\begin{tabular}{|c|c|c|c|c|c|c|c|c|c|c|c|c|c|c|c|c|c|c|c|c|c|c|c|}
\hline $\begin{array}{c}1 \\
220\end{array}$ & 210 & $\begin{array}{c}1 \\
200\end{array}$ & $\begin{array}{c}1 \\
190\end{array}$ & $\begin{array}{c}1 \\
180\end{array}$ & $\begin{array}{c}1 \\
170\end{array}$ & $\begin{array}{c}1 \\
160\end{array}$ & $\begin{array}{c}1 \\
150\end{array}$ & 140 & $\begin{array}{c}1 \\
130\end{array}$ & 120 & $\begin{array}{r}110 \\
\text { f1 }\end{array}$ & $\begin{array}{c}1 \\
100 \\
\mathrm{pm})\end{array}$ & 90 & 80 & $\begin{array}{l}7 \\
70\end{array}$ & 60 & 50 & $\begin{array}{c}1 \\
40\end{array}$ & 30 & 20 & 10 & $\begin{array}{l}1 \\
0\end{array}$ & -10 \\
\hline
\end{tabular}




\begin{tabular}{|ll|}
\hline \multicolumn{1}{|c|}{ Parameter } & \multicolumn{1}{c|}{ Value } \\
1 Title & youliang-6-IPr-AlkynylGoldComplex \\
2 Solvent & $\mathrm{CDCl} 3$ \\
3 Temperature & 25.0 \\
4 Relaxation Delay & 10.0000 \\
5 Spectrometer Frequency & 499.86 \\
\hline
\end{tabular}

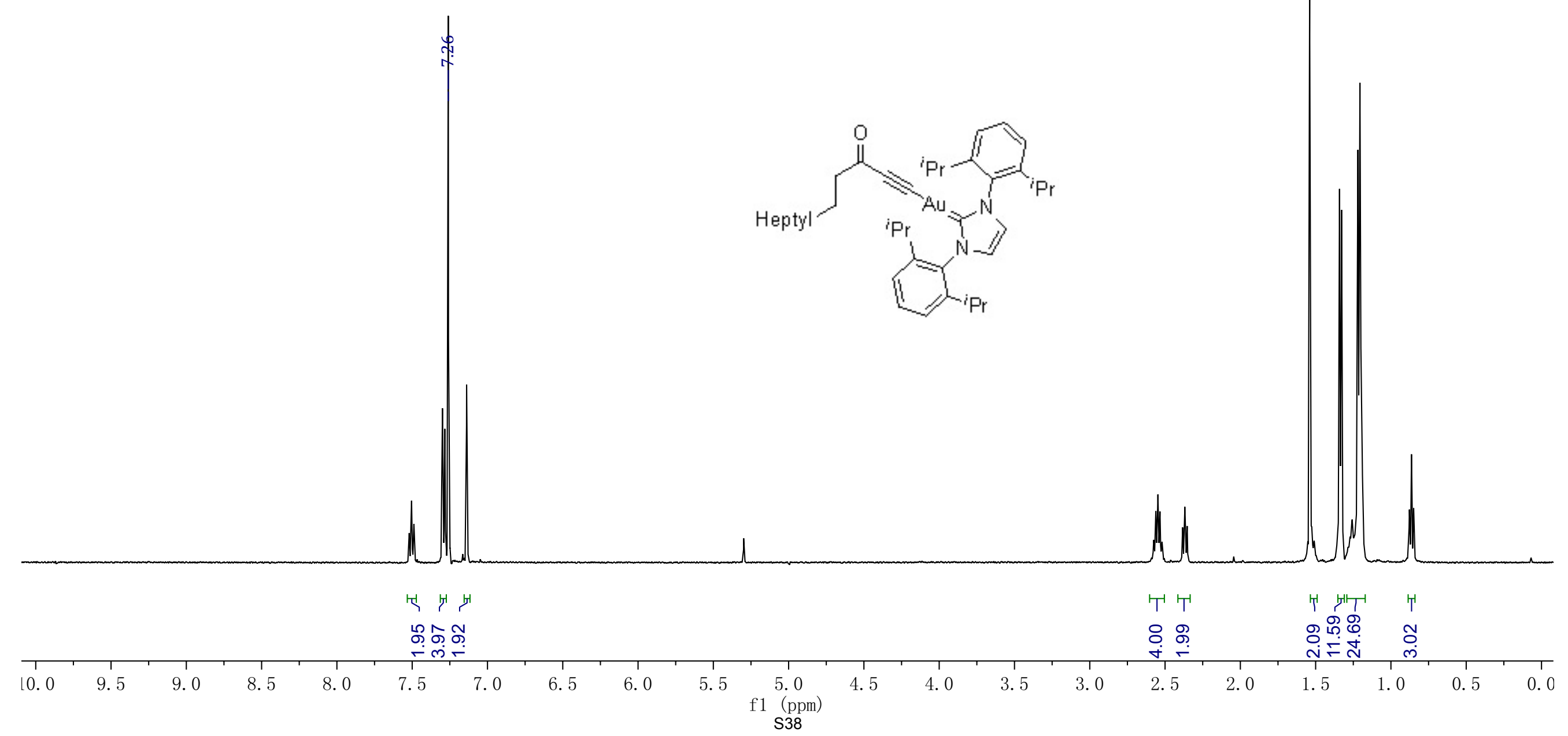




\begin{tabular}{|ll|}
\hline \multicolumn{1}{|c|}{ Parameter } & \multicolumn{1}{c|}{ Value } \\
1 Title & youliang-6-Alkynylgold-C13k \\
2 Solvent & $\mathrm{CDCl} 3$ \\
3 Temperature & 25.0 \\
4 Relaxation Delay & 1.0000 \\
5 Spectrometer Frequency & 125.70 \\
\hline
\end{tabular}

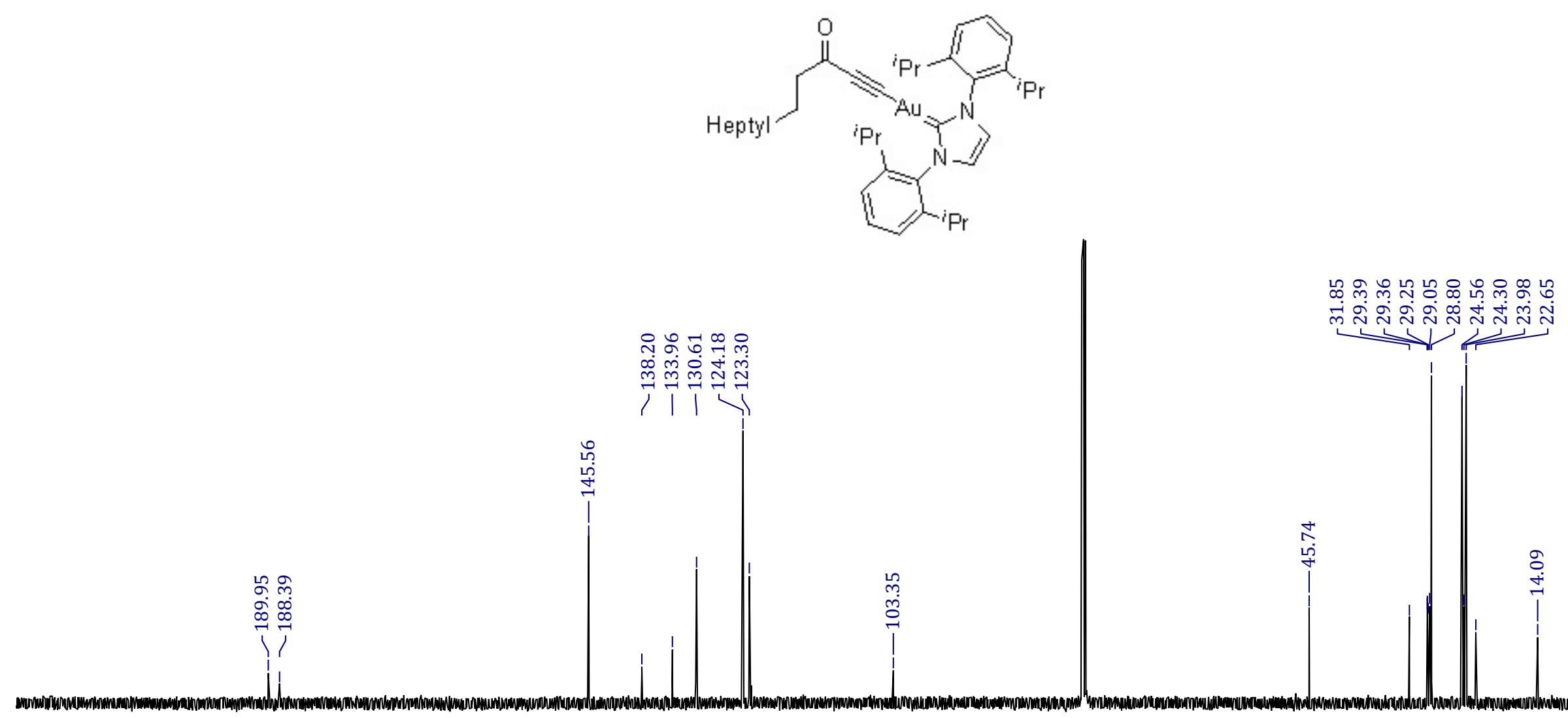

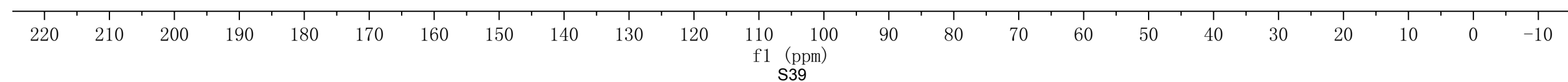




\begin{tabular}{|ll|}
\hline \multicolumn{2}{|c|}{ Parameter } \\
1 Title & youliang-6-66A-P-bromoalkyne \\
2 Solvent & $\mathrm{CDCl} 3$ \\
3 Spectrometer Frequency & 499.86 \\
4 Nucleus & $1 \mathrm{H}$ \\
\hline
\end{tabular}
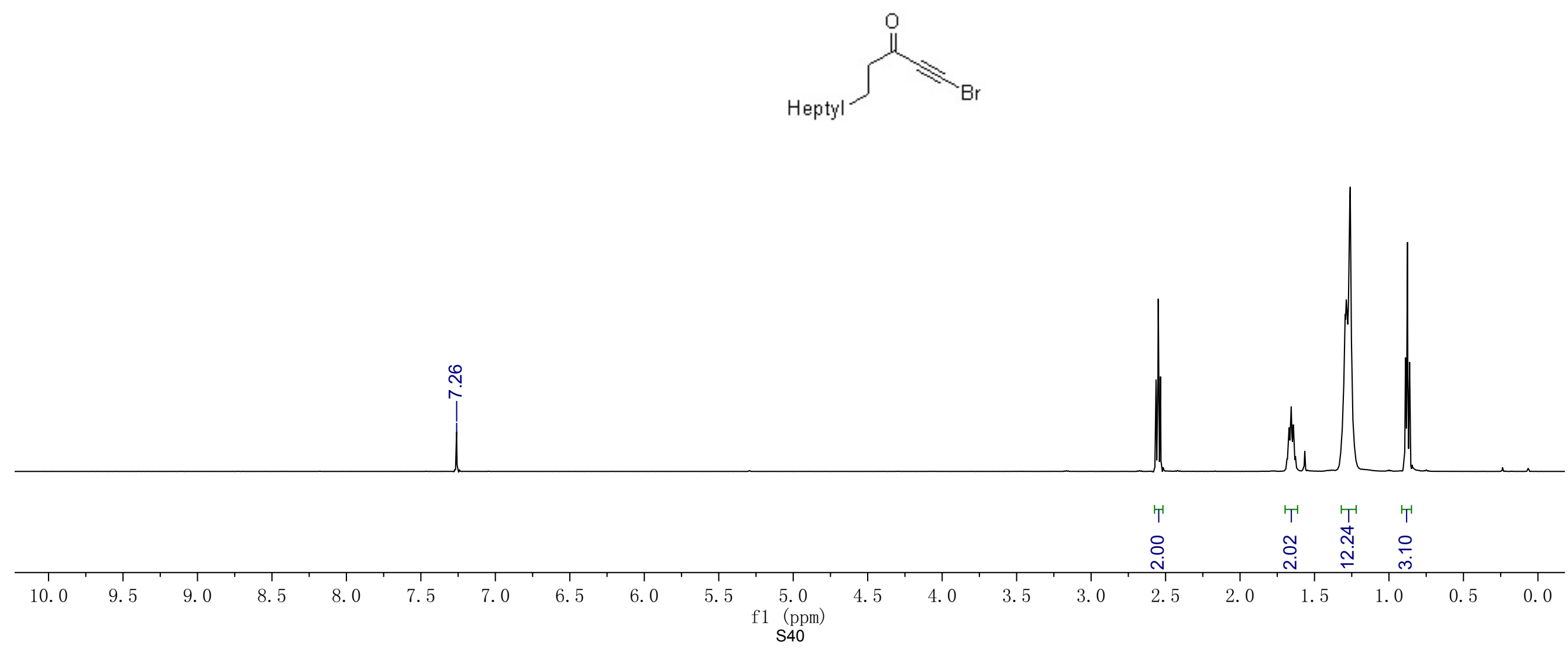


\begin{tabular}{|c|c|}
\hline Parameter & Value \\
\hline 1 Title & youliang-6-66A-P-bromoalkyne-C13i \\
\hline 2 Solvent & $\mathrm{CDCl} 3$ \\
\hline 3 Spectrometer Frequency & 125.70 \\
\hline 4 Nucleus & $13 \mathrm{C}$ \\
\hline
\end{tabular}
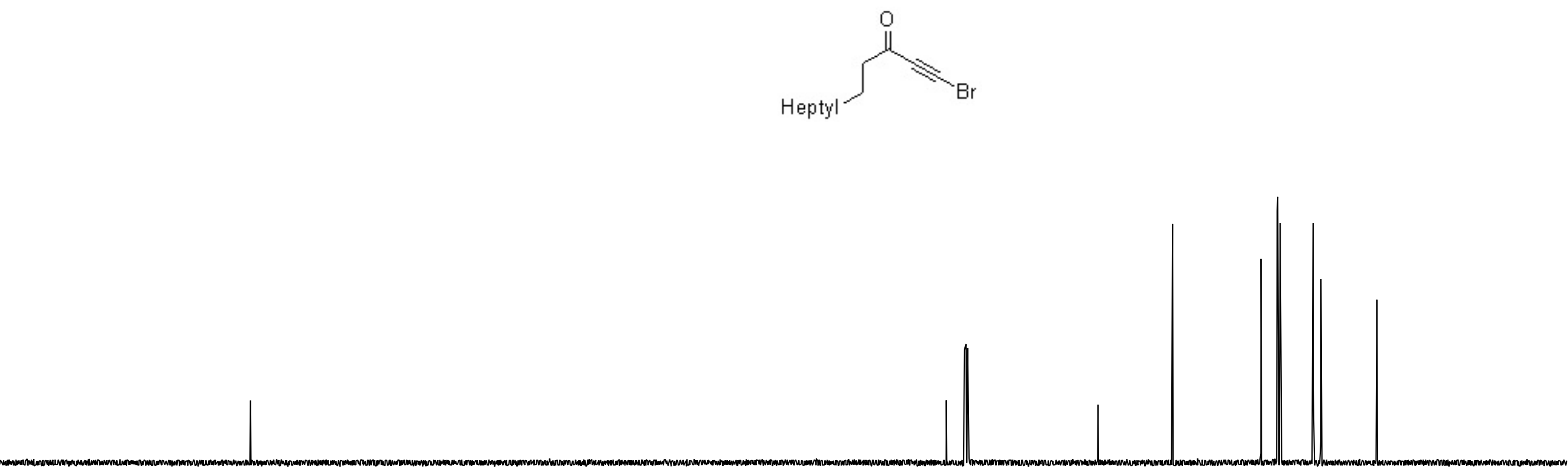

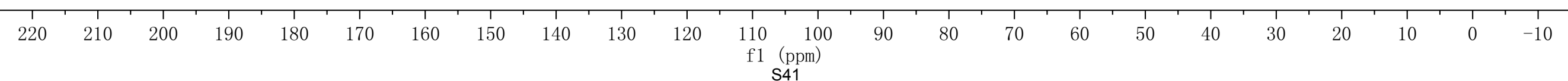




\begin{tabular}{|ll|}
\hline \multicolumn{1}{|c|}{ Parameter } & \multicolumn{1}{c|}{ Value } \\
1 Title & youliang-6-11-A-P \\
2 Solvent & CDCl3 \\
3 Spectrometer Frequency & 499.86 \\
4 Nucleus & $1 \mathrm{H}$ \\
\hline
\end{tabular}
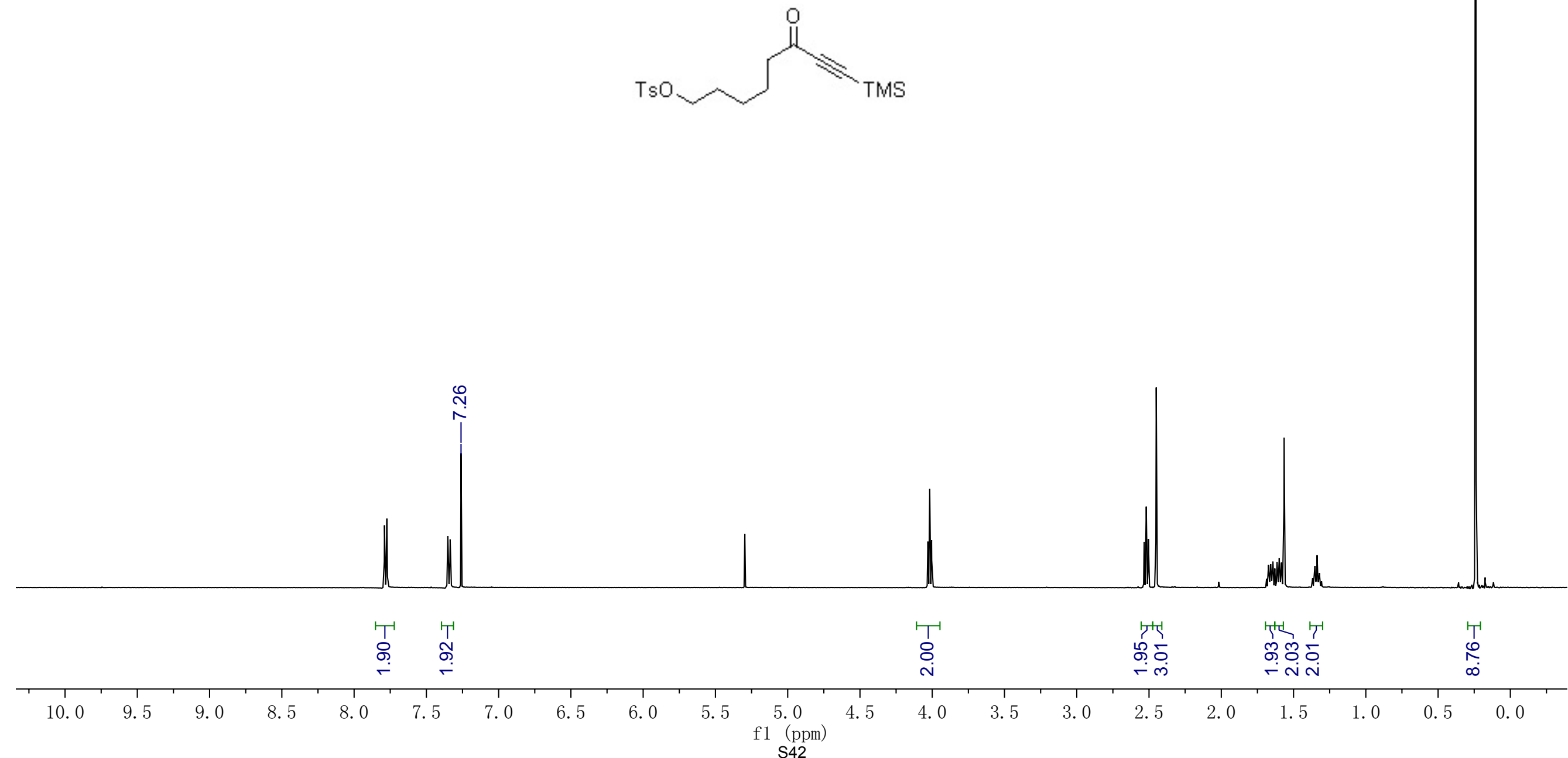


\begin{tabular}{|ll|}
\hline \multicolumn{1}{|c|}{ Parameter } & \multicolumn{1}{c|}{ Value } \\
1 Title & youliang-6-11-A-P-C13f \\
2 Solvent & CDCl3 \\
3 Spectrometer Frequency & 125.70 \\
4 Nucleus & $13 \mathrm{C}$ \\
\hline
\end{tabular}
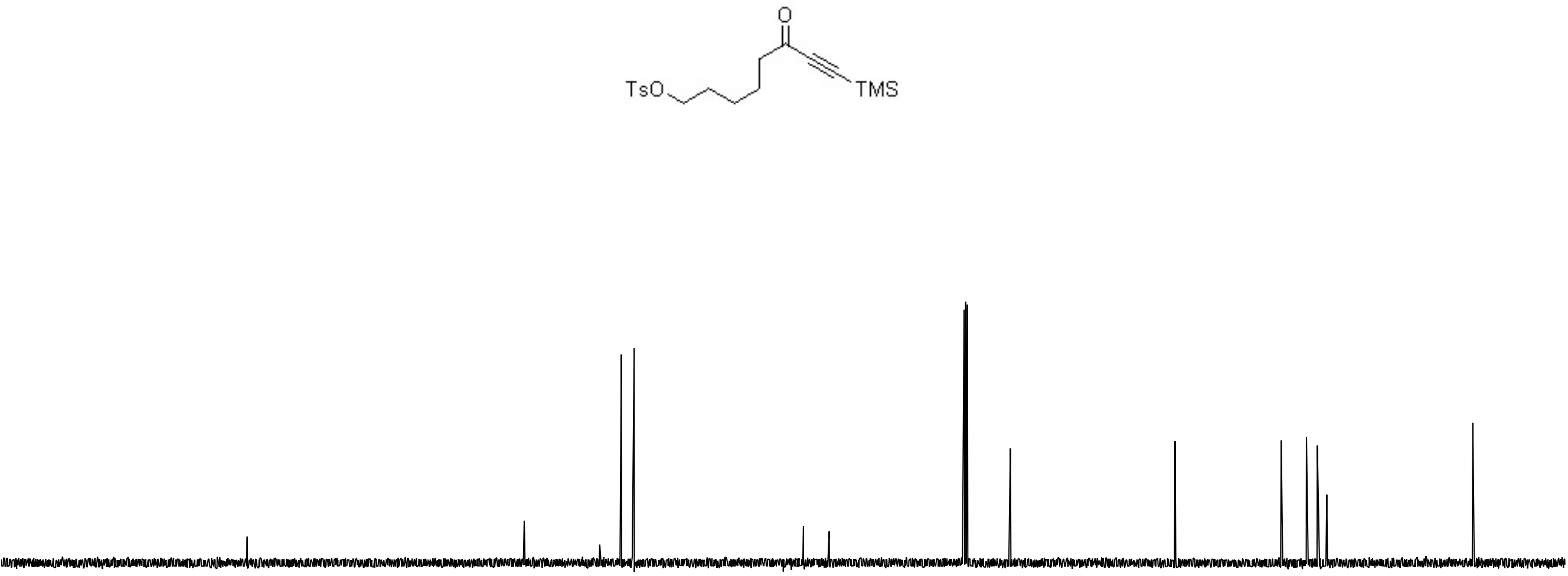

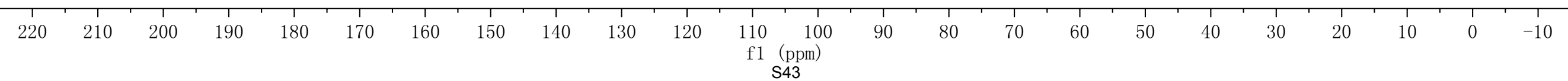




\begin{tabular}{|ll|}
\hline \multicolumn{1}{|c|}{ Parameter } & \multicolumn{1}{c|}{ Value } \\
1 Title & youliang-6-15B-P \\
2 Solvent & CDCl3 \\
3 Temperature & 25.0 \\
4 Relaxation Delay & 10.0000 \\
5 Spectrometer Frequency & 499.86 \\
\hline
\end{tabular}
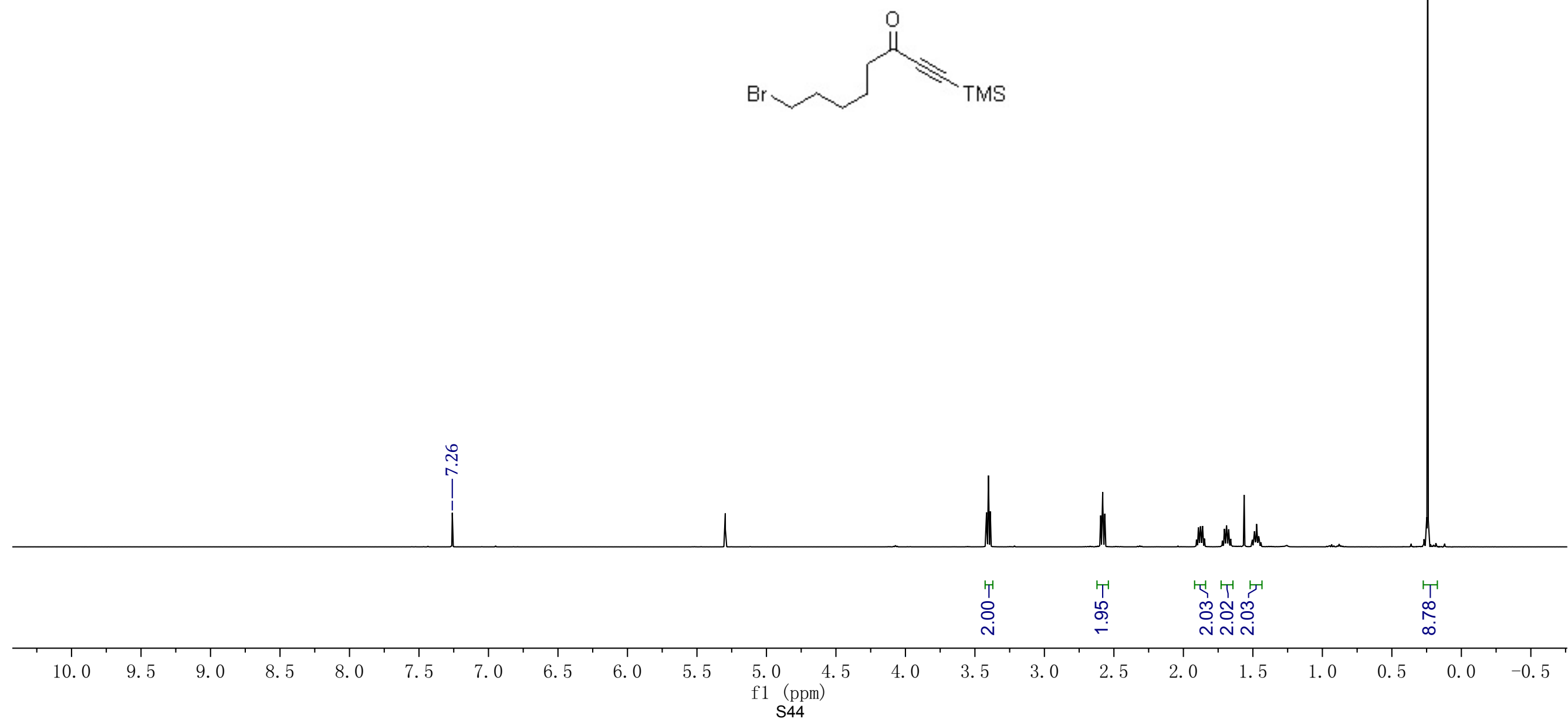


\begin{tabular}{|ll|}
\hline \multicolumn{1}{|c|}{ Parameter } & \multicolumn{1}{c|}{ Value } \\
1 Title & youliang-6-15B-P-C13g \\
2 Solvent & $\mathrm{CDCl3}$ \\
3 Temperature & 25.0 \\
4 Relaxation Delay & 1.0000 \\
5 Spectrometer Frequency & 125.70 \\
\hline
\end{tabular}
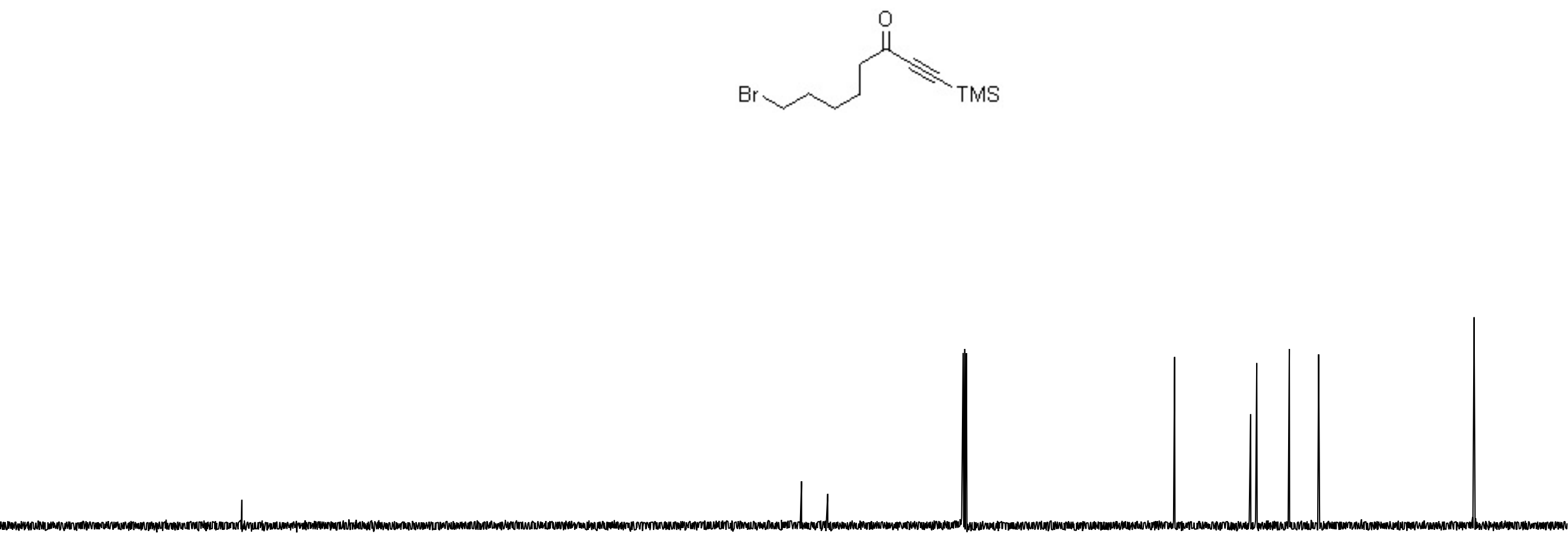

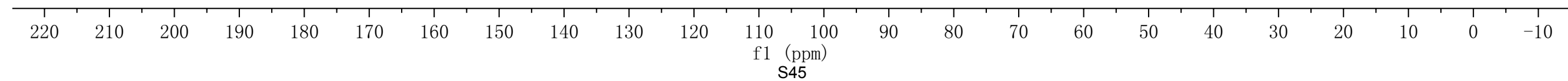




\begin{tabular}{|ll|}
\hline \multicolumn{1}{|c|}{ Parameter } & \multicolumn{1}{c|}{ Value } \\
1 Title & youliang-5-275-A-SM \\
2 Solvent & CDCl3 \\
3 Temperature & 25.0 \\
4 Relaxation Delay & 10.0000 \\
5 Spectrometer Frequency & 499.86 \\
\hline
\end{tabular}
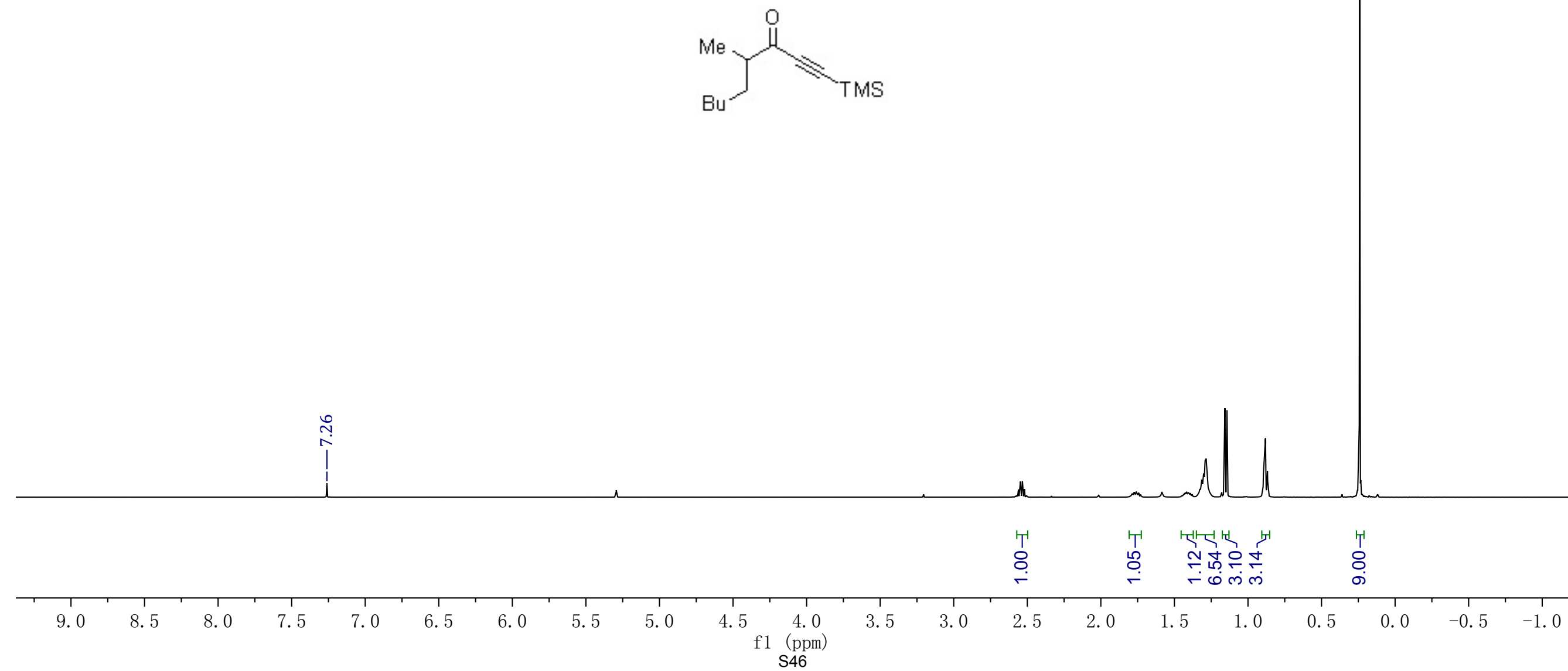


\begin{tabular}{|ll|}
\hline \multicolumn{1}{|c|}{ Parameter } & \multicolumn{1}{c|}{ Value } \\
1 Title & youliang-5-275-A-SM-C13i \\
2 Solvent & CDCl3 \\
3 Temperature & 25.0 \\
4 Relaxation Delay & 1.0000 \\
5 Spectrometer Frequency & 125.70 \\
\hline
\end{tabular}

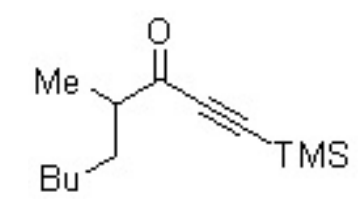

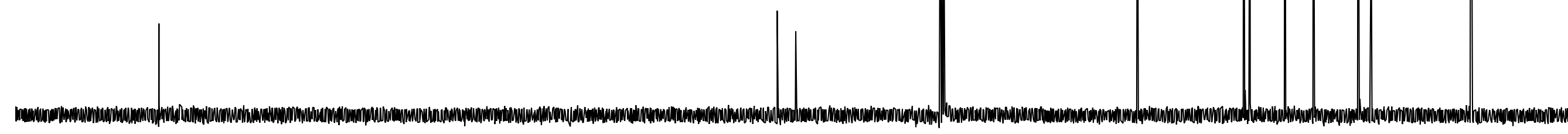

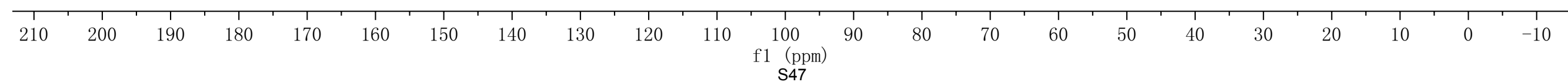




\begin{tabular}{|ll|}
\hline \multicolumn{1}{|c|}{ Parameter } & \multicolumn{1}{c|}{ Value } \\
1 Title & youliang-6-25B-SM \\
2 Solvent & $\mathrm{CDCl} 3$ \\
3 Spectrometer Frequency & 499.86 \\
4 Nucleus & $1 \mathrm{H}$ \\
\hline
\end{tabular}
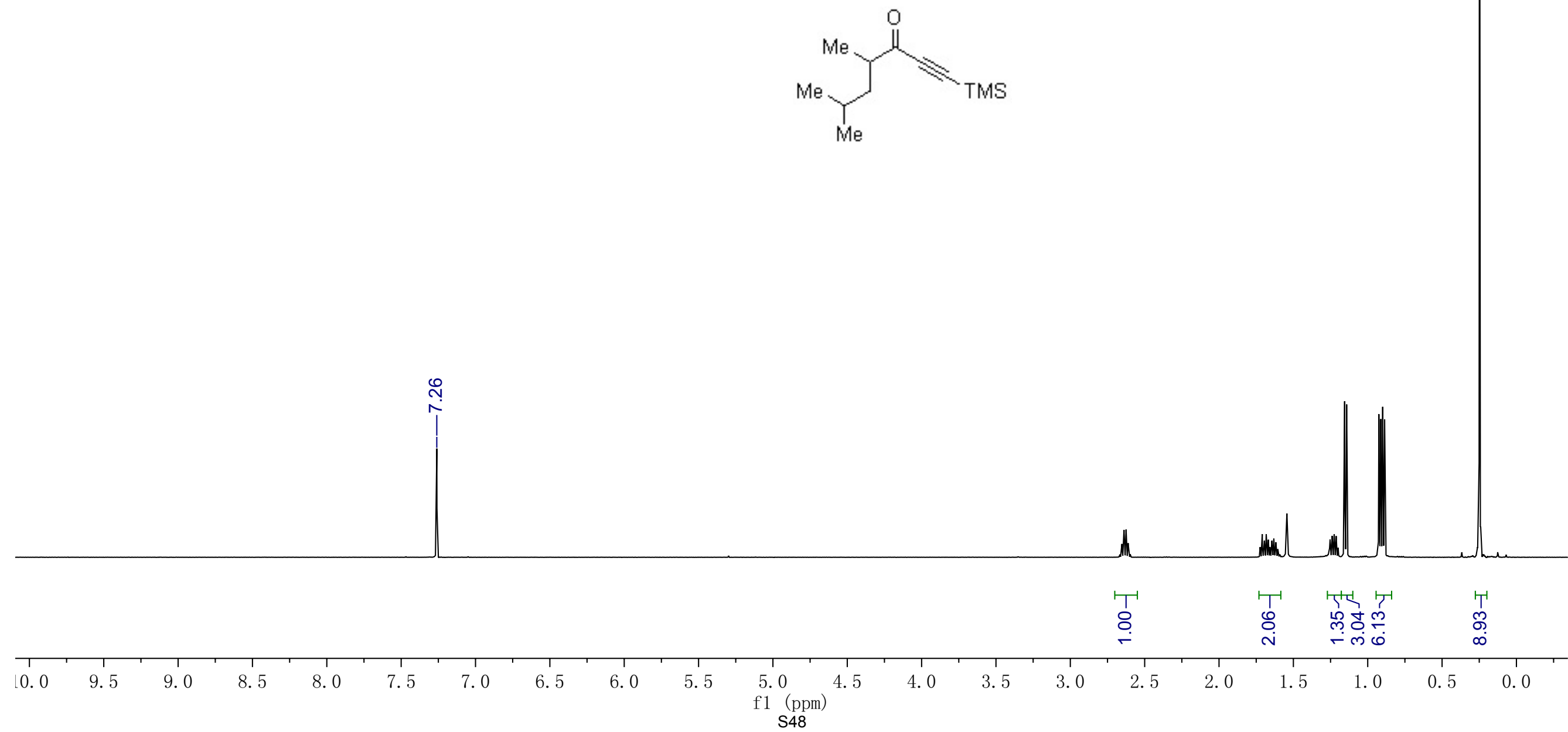
2 Solvent

iliang-6-25B-SM-C13h

(25.70
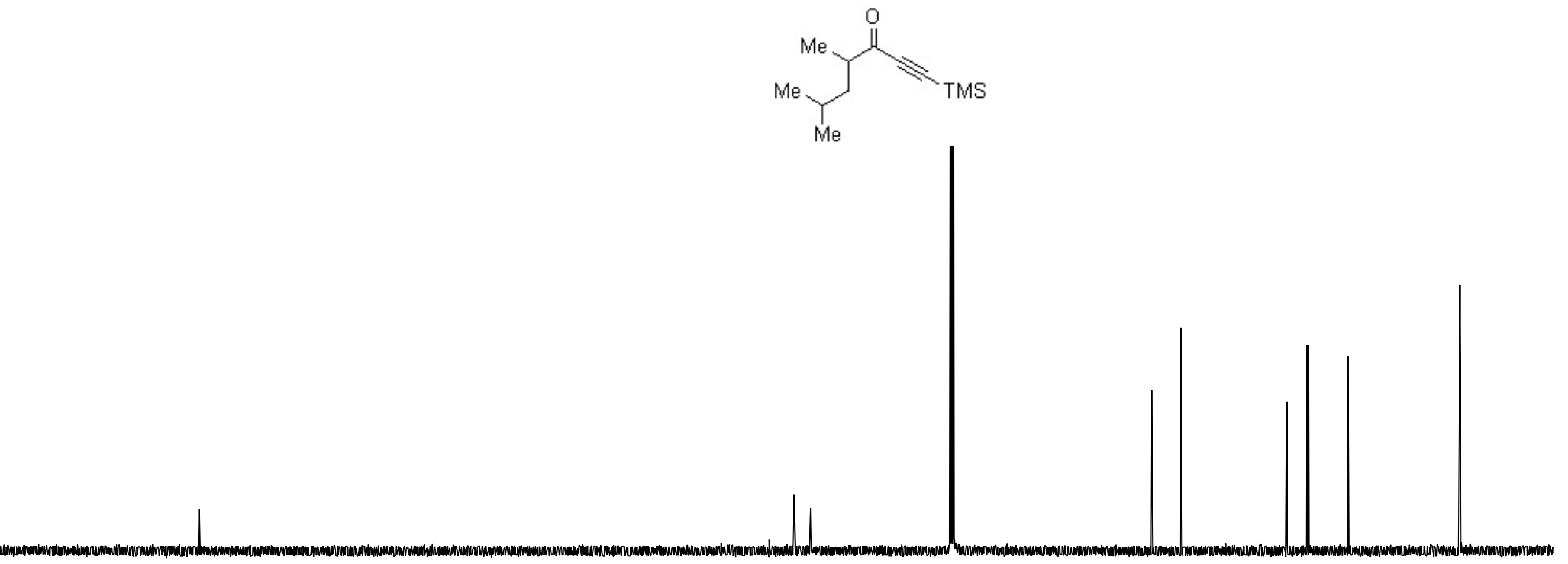

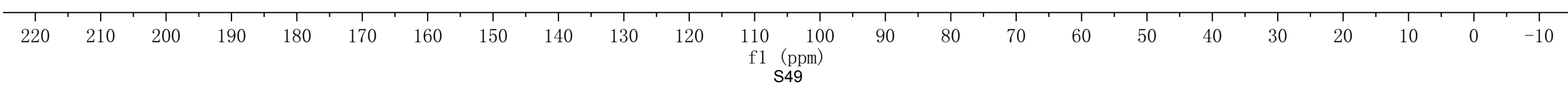




\begin{tabular}{|ll|}
\hline \multicolumn{1}{|c|}{ Parameter } & \multicolumn{1}{c|}{ Value } \\
1 Title & youliang-6-22-A-P-again \\
2 Solvent & cdc13 \\
3 Spectrometer Frequency & 399.78 \\
4 Nucleus & $1 \mathrm{H}$ \\
\hline
\end{tabular}
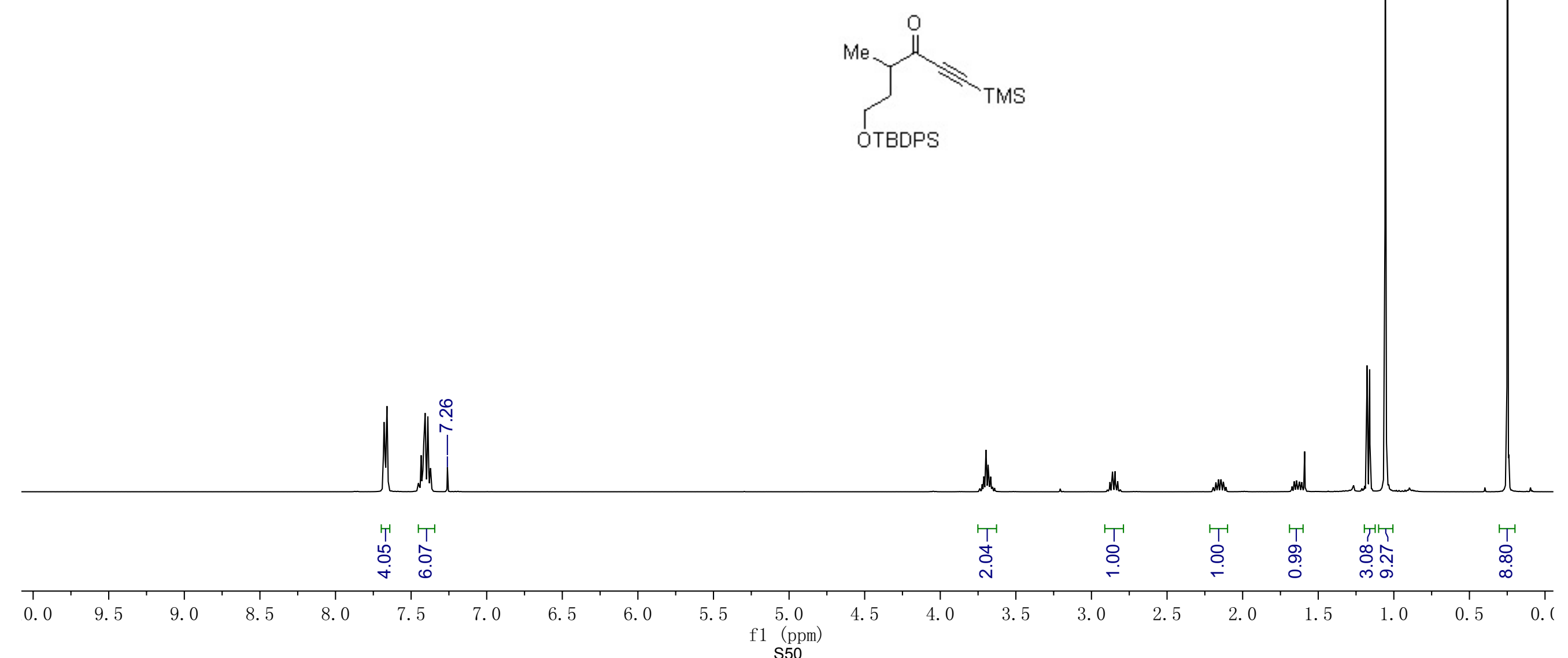


\begin{tabular}{|ll|}
\hline \multicolumn{1}{|c|}{ Parameter } & \multicolumn{1}{c|}{ Value } \\
1 Title & youliang-6-22A-P-C13j \\
2 Solvent & $\mathrm{CDCl} 3$ \\
3 Spectrometer Frequency & 125.70 \\
4 Nucleus & $13 \mathrm{C}$ \\
\hline
\end{tabular}

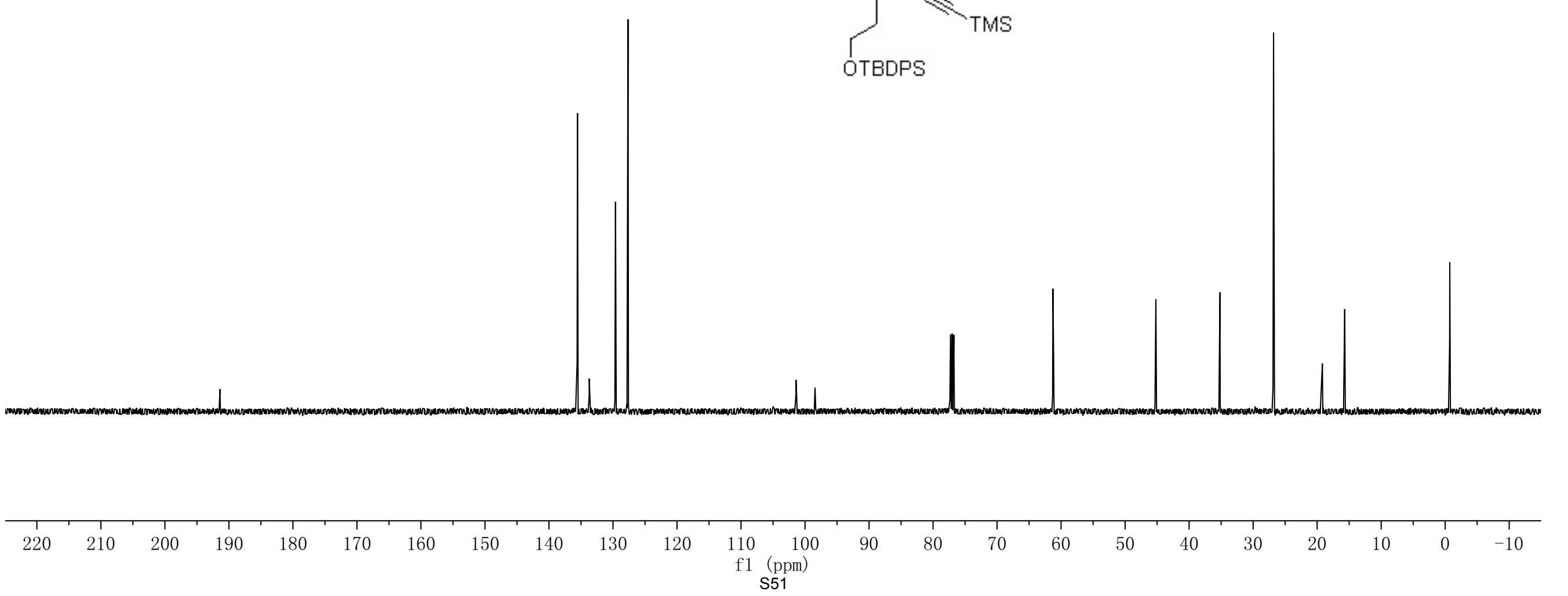




\begin{tabular}{|ll|}
\hline \multicolumn{1}{|c|}{ Parameter } & \multicolumn{1}{c|}{ Value } \\
1 Title & youliang-6-44B-SM \\
2 Solvent & $\mathrm{CDCl} 3$ \\
3 Spectrometer Frequency & 499.86 \\
4 Nucleus & $1 \mathrm{H}$ \\
\hline
\end{tabular}
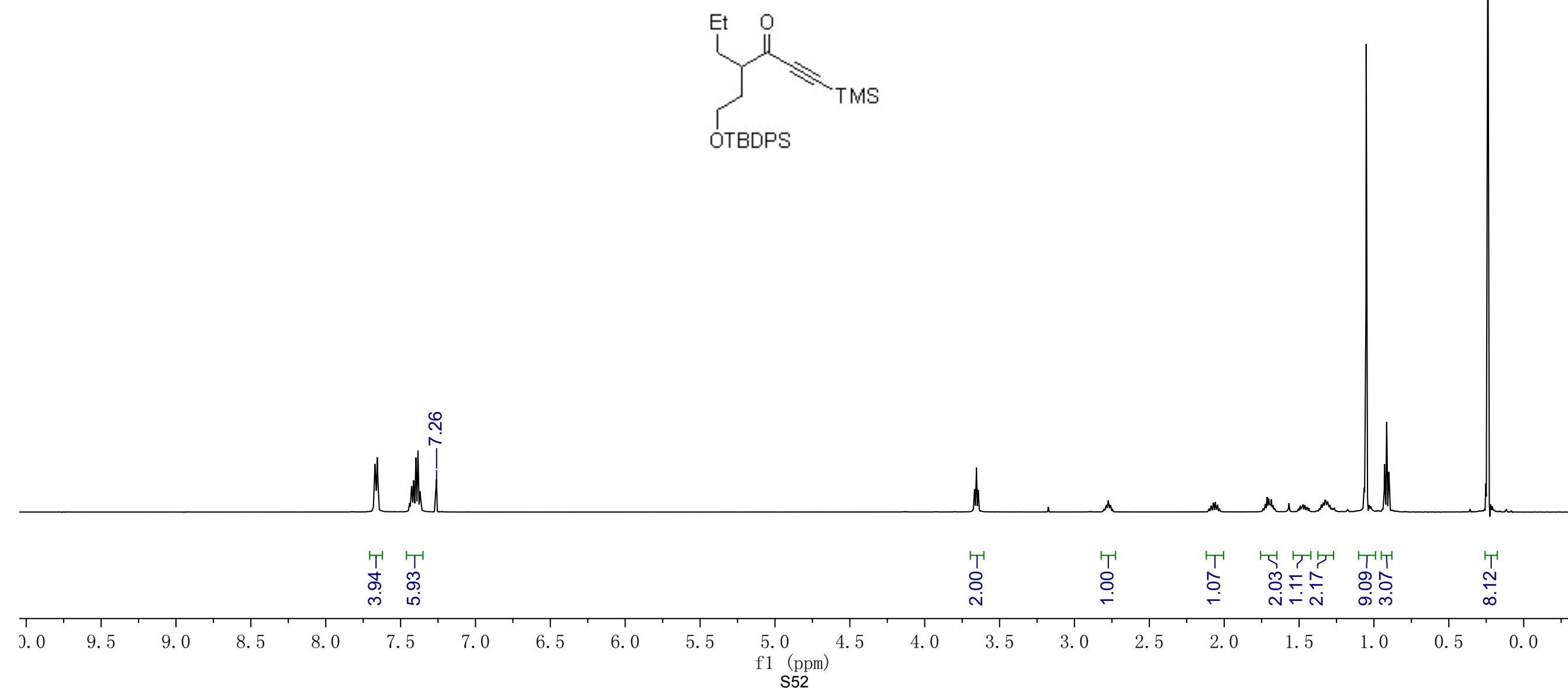


\begin{tabular}{|ll|}
\hline \multicolumn{2}{|c|}{ Parameter } \\
1 Title & youliang-6-44B-SM-C13b \\
2 Solvent & CDCl3 \\
3 Spectrometer Frequency & 125.70 \\
4 Nucleus & $13 \mathrm{C}$ \\
\hline
\end{tabular}
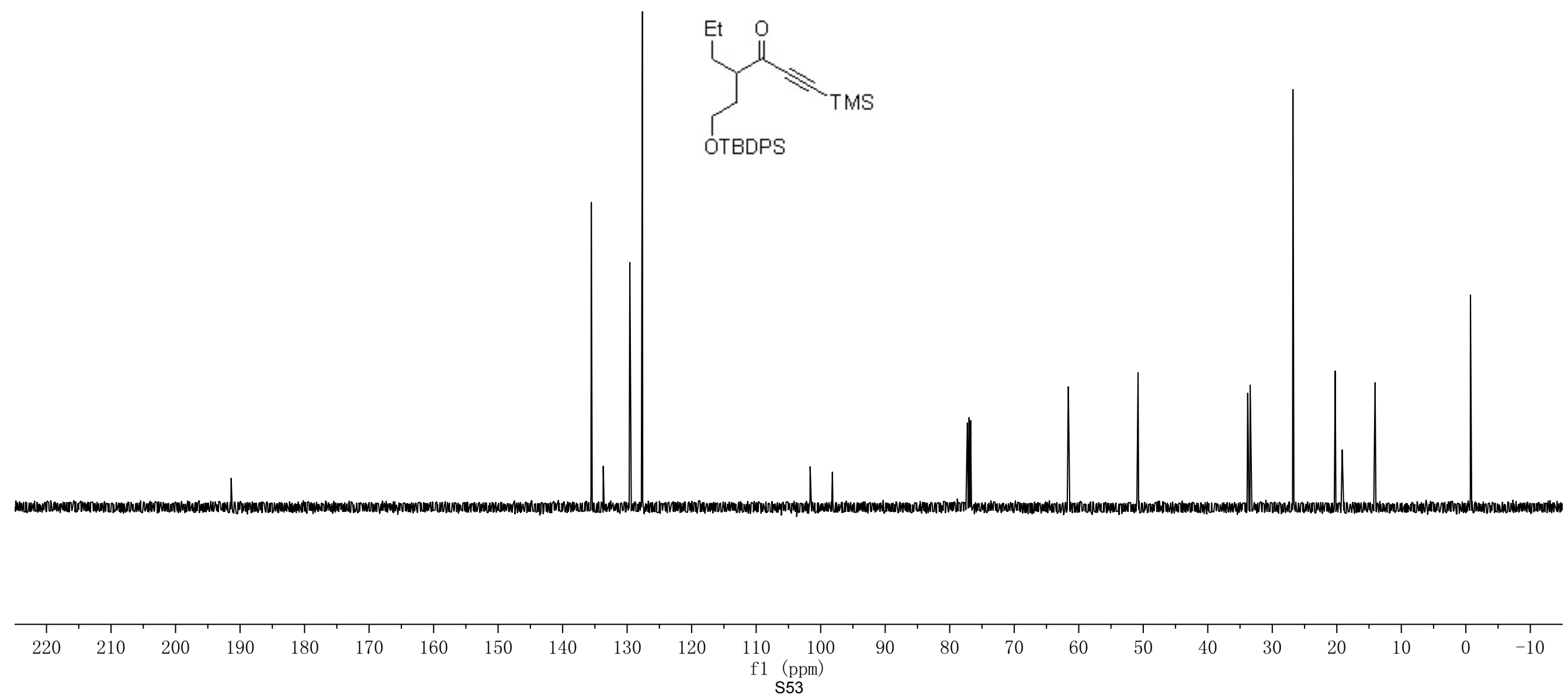


\begin{tabular}{|ll|}
\hline \multicolumn{1}{|c|}{ Parameter } & \multicolumn{1}{c|}{ Value } \\
1 Title & youliang-6-74A-SM \\
2 Solvent & $\mathrm{CDCl} 3$ \\
3 Spectrometer Frequency & 499.86 \\
4 Nucleus & $1 \mathrm{H}$ \\
\hline
\end{tabular}

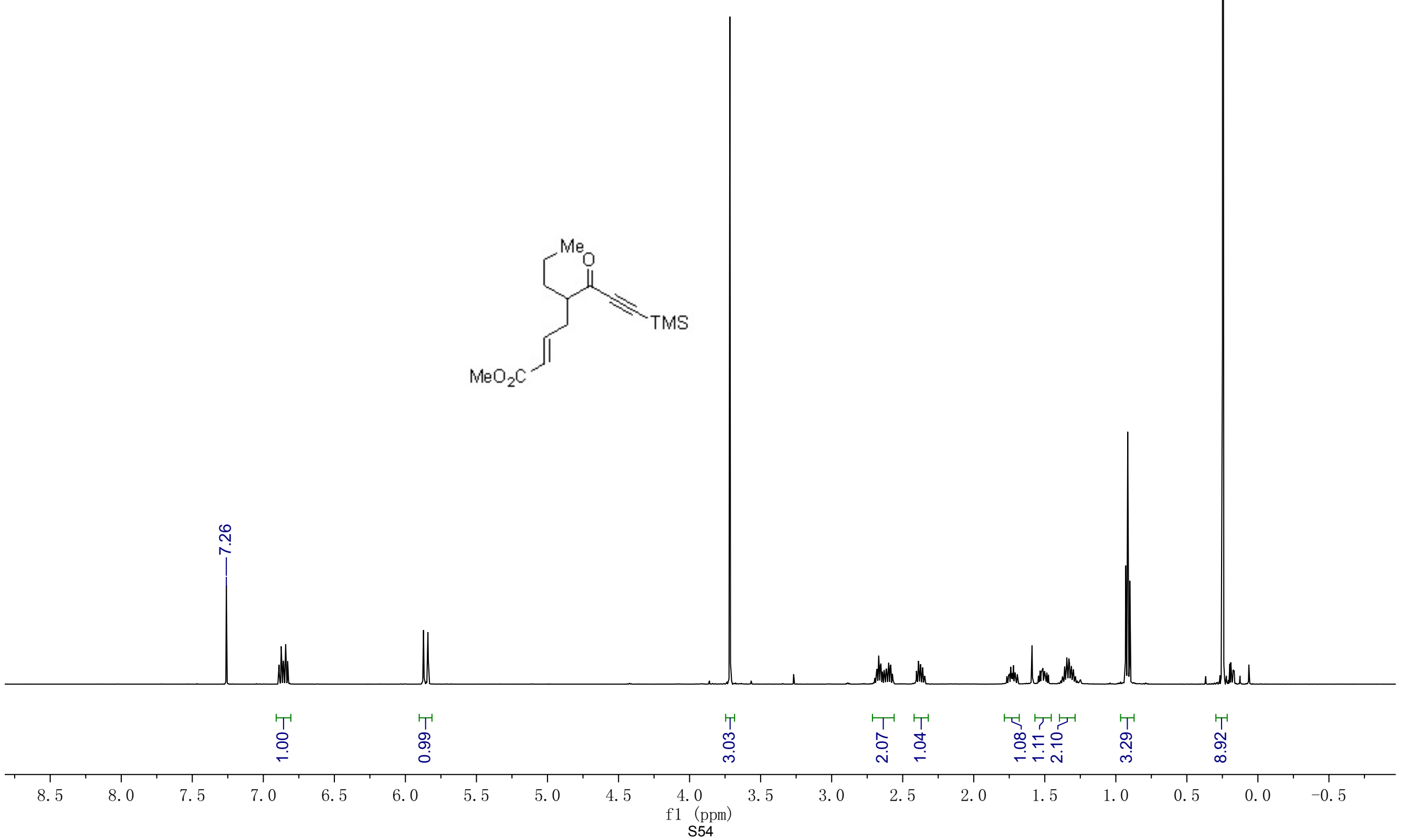


3 Spectrometer Frequency 125.70

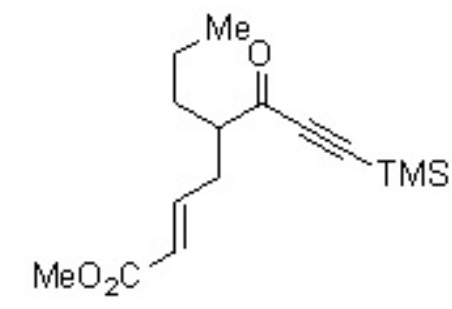

$\mathrm{MeO}_{2} \mathrm{C}$

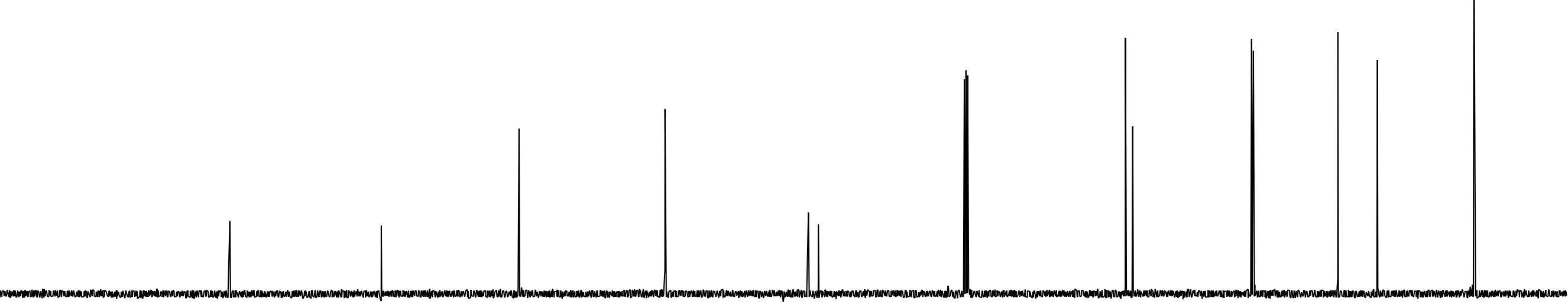

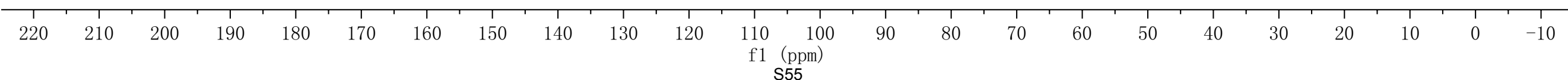




\begin{tabular}{|ll|}
\hline \multicolumn{1}{|c|}{ Parameter } & \multicolumn{1}{c|}{ Value } \\
1 Title & youliang-6-48B-SM \\
2 Solvent & CDCl3 \\
3 Spectrometer Frequency & 499.86 \\
4 Nucleus & $1 \mathrm{H}$ \\
\hline
\end{tabular}
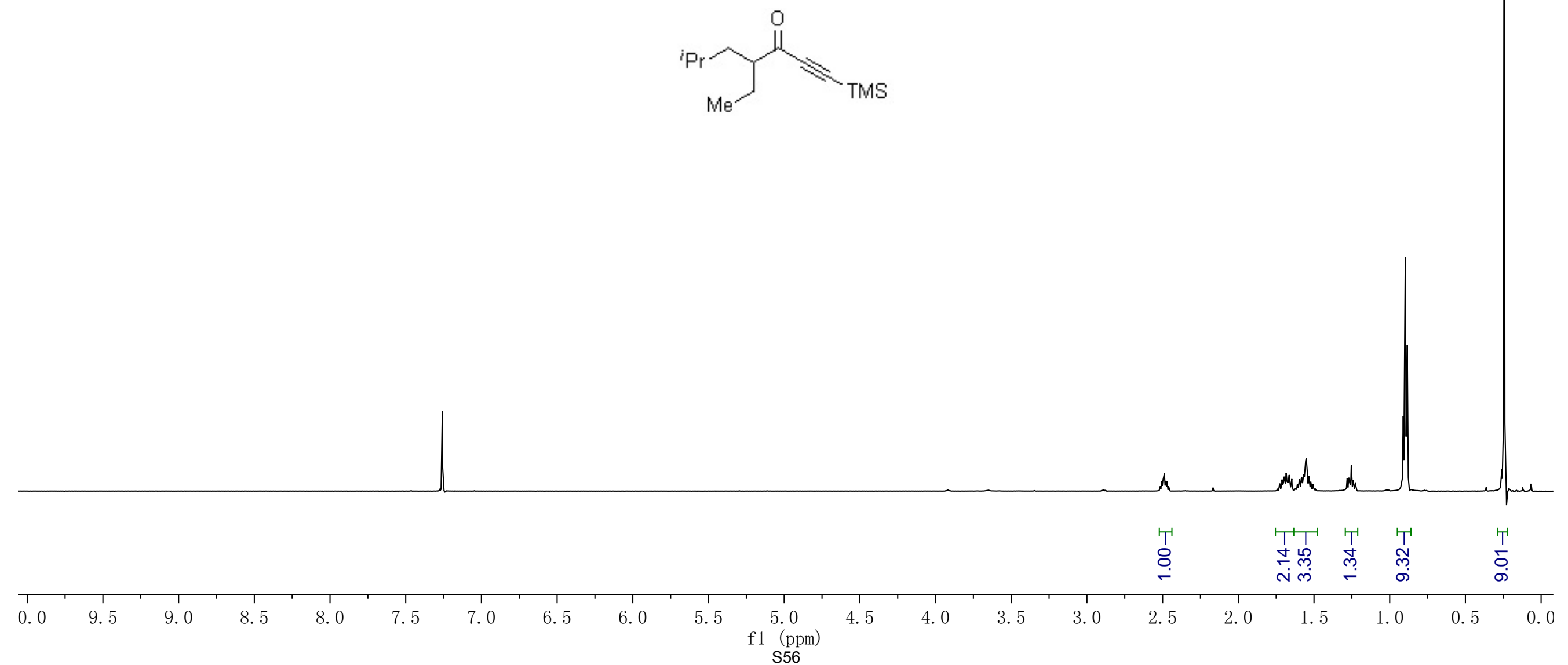


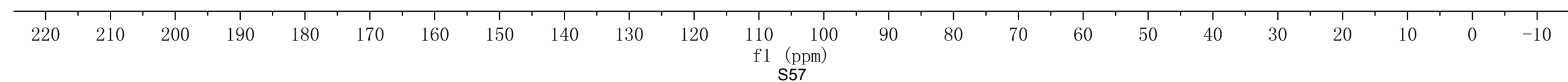




\begin{tabular}{|c|c|}
\hline Parameter & Value \\
\hline 1 Title & youliang-6-41A-P-agair \\
\hline 2 Solvent & $\mathrm{CDCl} 3$ \\
\hline 3 Spectrometer Frequen & y 499.86 \\
\hline 4 Nucleus & $1 \mathrm{H}$ \\
\hline
\end{tabular}
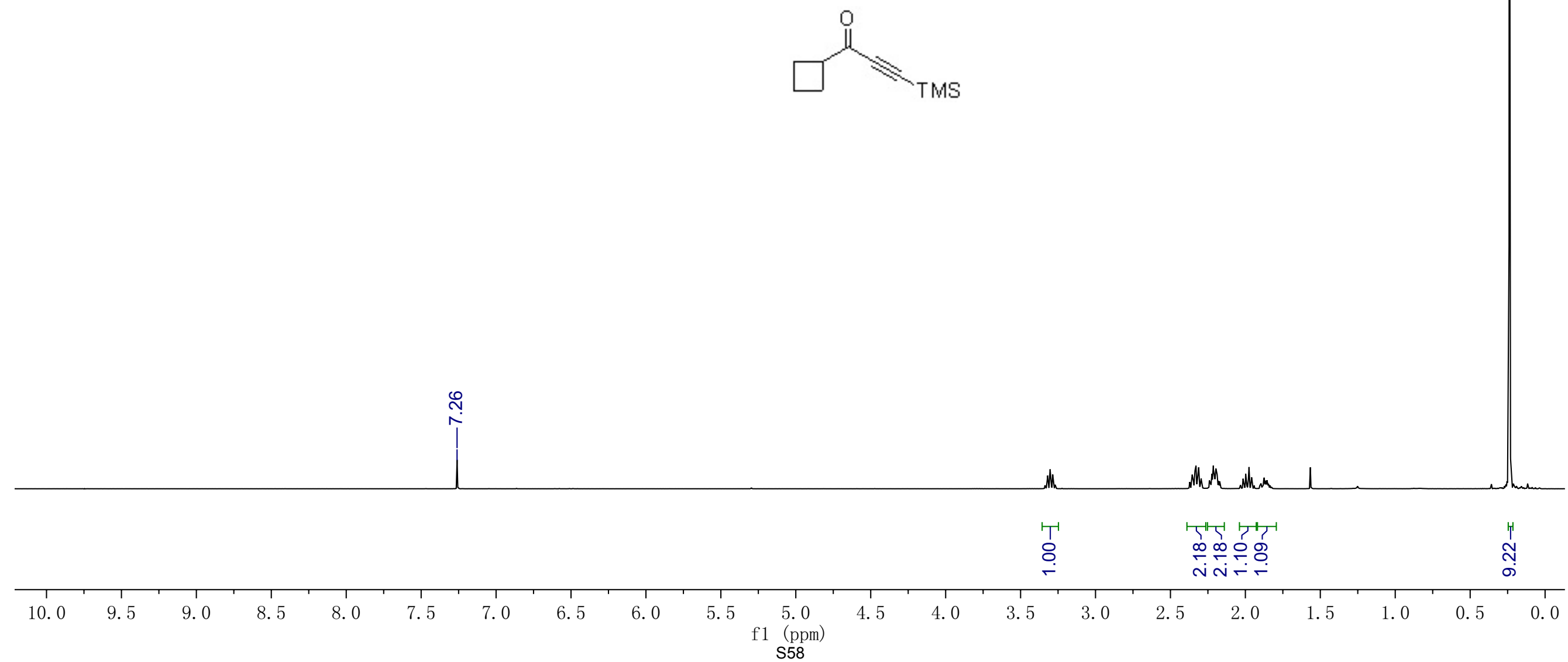
Spectrometer Frequency 125.70
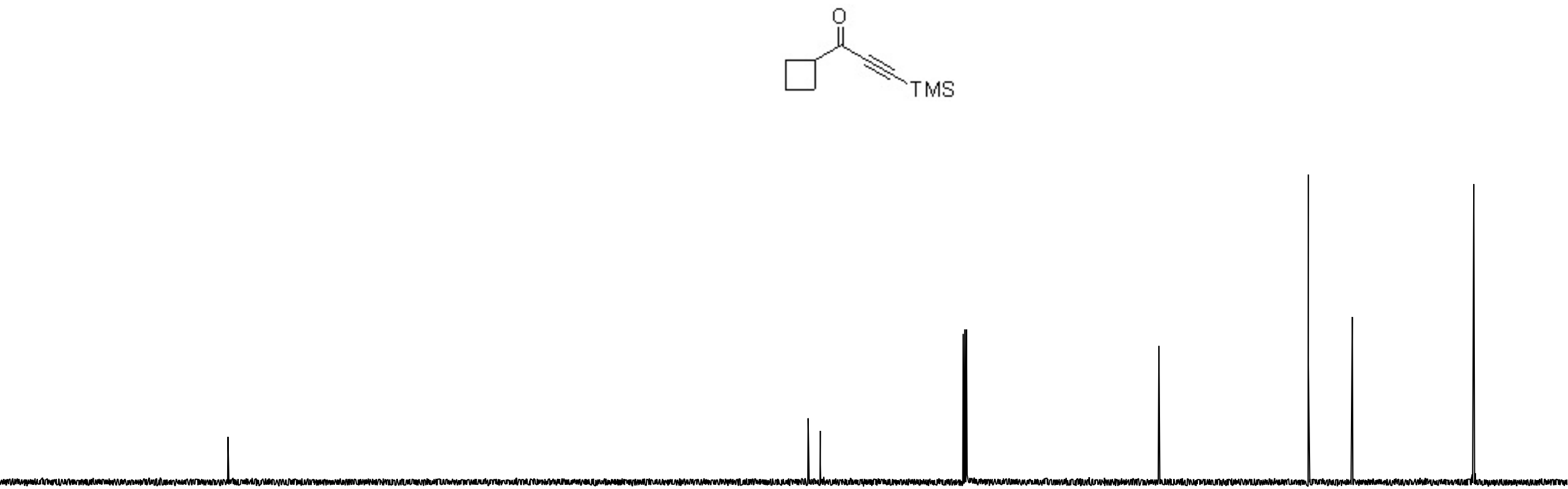

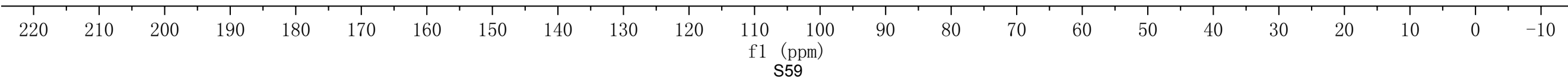




\begin{tabular}{|ll|}
\hline \multicolumn{1}{|c|}{ Parameter } & \multicolumn{1}{c|}{ Value } \\
1 Title & Max-85-pure2 \\
2 Solvent & $\mathrm{CDCl} 3$ \\
3 Temperature & 25.0 \\
4 Relaxation Delay & 4.8000 \\
5 Spectrometer Frequency & 499.86 \\
\hline
\end{tabular}
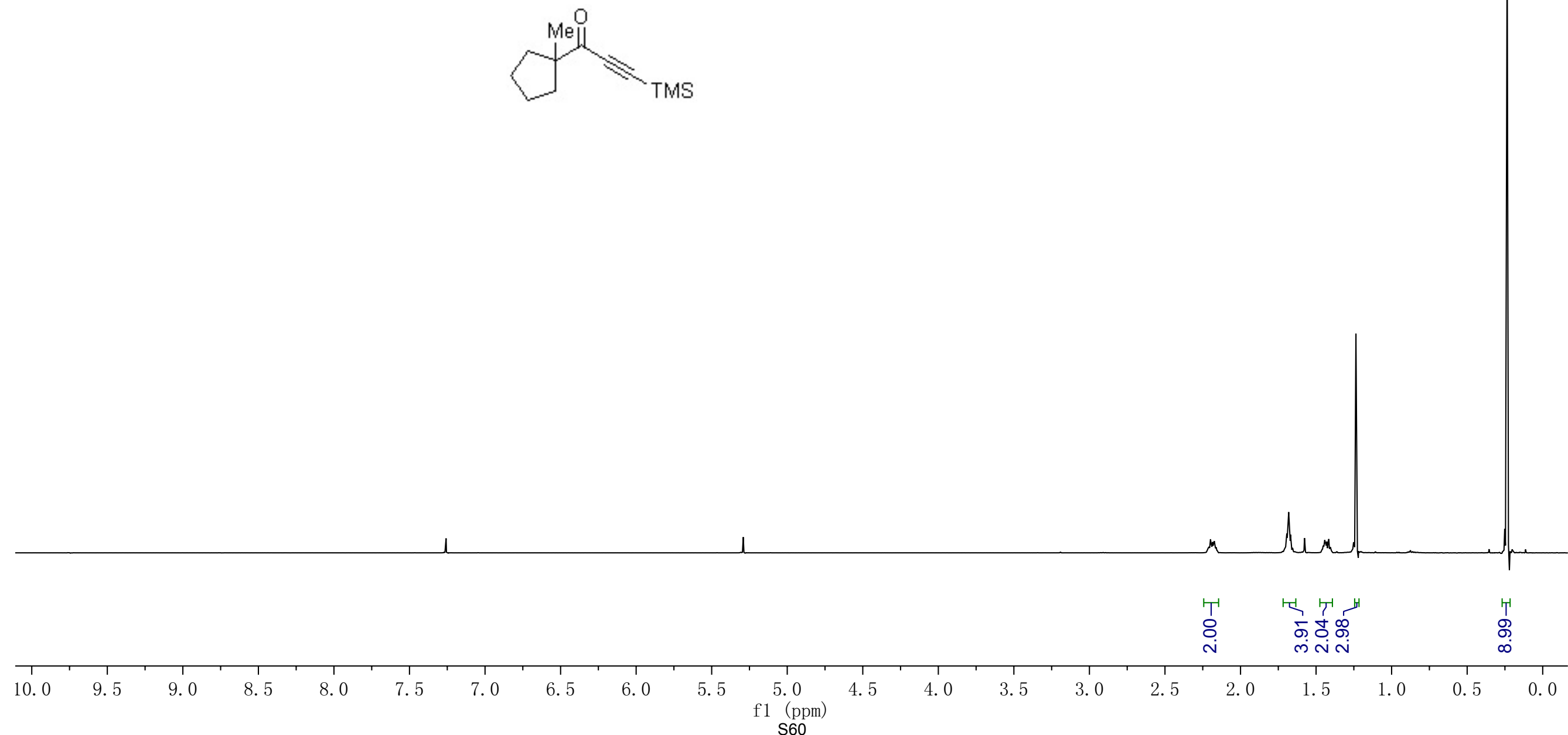


\begin{tabular}{|ll|}
\hline \multicolumn{1}{|c|}{ Parameter } & \multicolumn{1}{c|}{ Value } \\
1 Title & Max-85-carbon2 \\
2 Solvent & CDCl3 \\
3 Temperature & 25.0 \\
4 Relaxation Delay & 1.0000 \\
5 Spectrometer Frequency & 125.70 \\
\hline
\end{tabular}
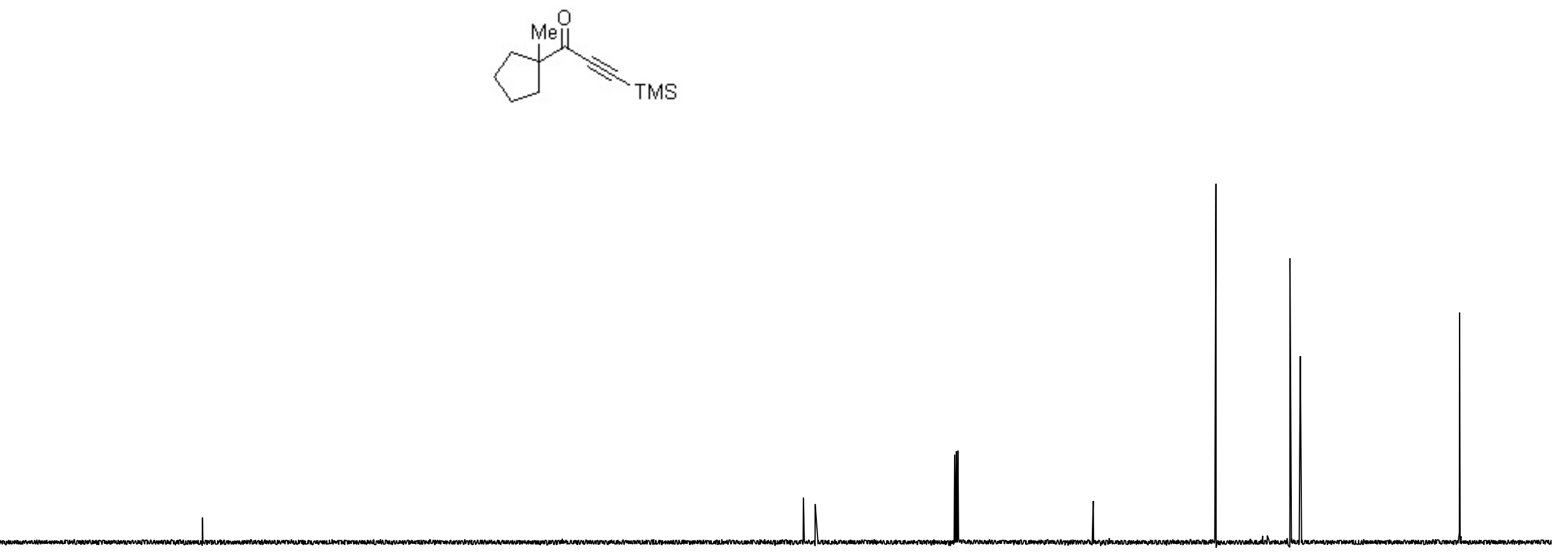

\begin{tabular}{|c|c|c|c|c|c|c|c|c|c|c|c|c|c|c|c|c|c|c|c|c|c|c|c|c|c|}
\hline 220 & 210 & 200 & 190 & 180 & 170 & 160 & 150 & 140 & 130 & 120 & $\begin{array}{r}110 \\
\mathrm{f} 1\end{array}$ & $\begin{array}{l}100 \\
\text { ppm) } \\
61\end{array}$ & 9 & 8 & 0 & 70 & 60 & 50 & & 40 & 30 & 20 & 10 & 0 & -10 \\
\hline
\end{tabular}




\begin{tabular}{|ll|}
\hline \multicolumn{2}{|c|}{ Parameter } \\
1 Title & youliang-5-299-C-SM-again \\
2 Solvent & $\mathrm{CDCl} 3$ \\
3 Spectrometer Frequency & 499.86 \\
4 Nucleus & $1 \mathrm{H}$ \\
\hline
\end{tabular}
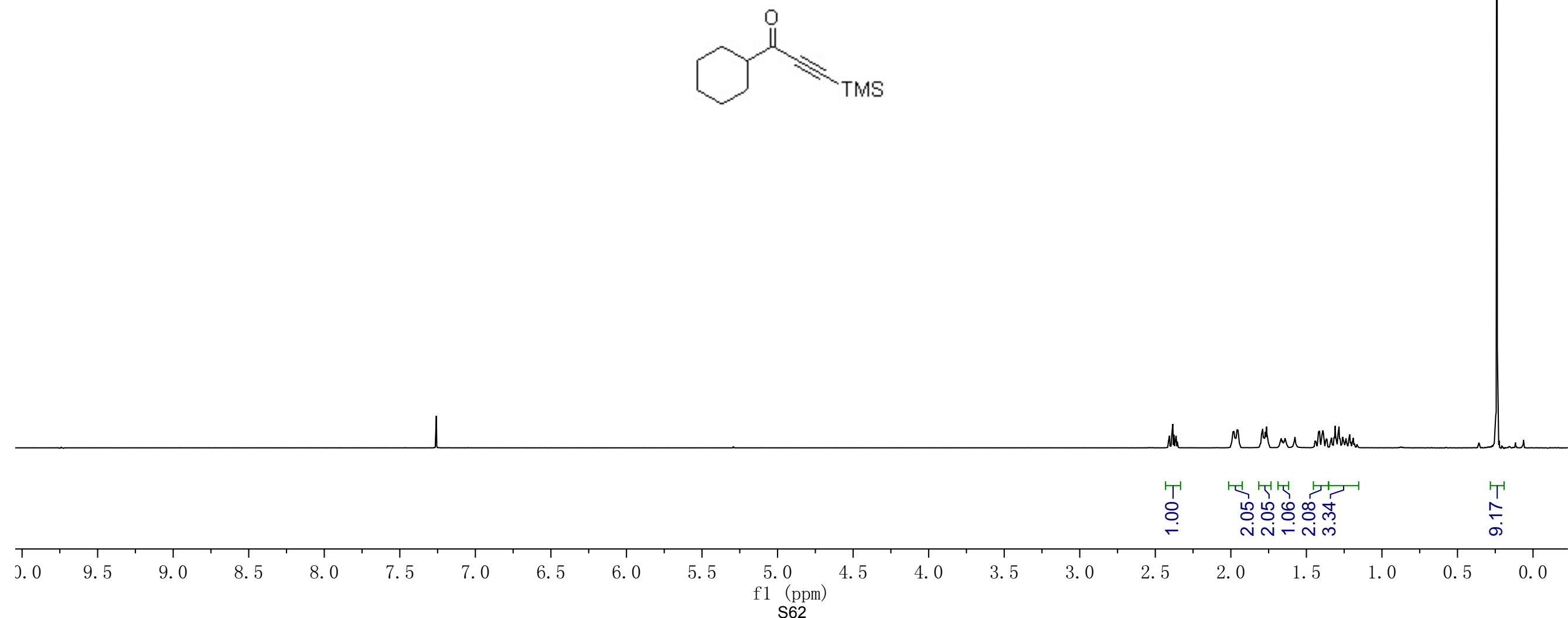
2 Solvent

youliang-5-299-C-SM-again-C13a

Frometer Frequency 125.70

4 Nucleus $13 \mathrm{C}$

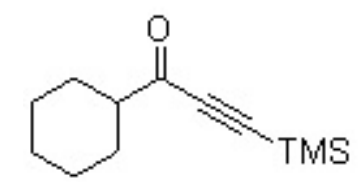

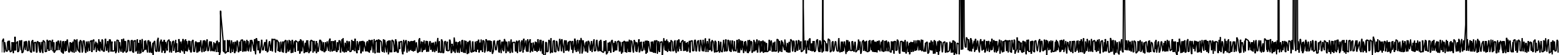

\begin{tabular}{|c|c|c|c|c|c|c|c|c|c|c|c|c|c|c|c|c|c|c|c|c|c|c|c|}
\hline $\begin{array}{c}1 \\
220\end{array}$ & 210 & 200 & $\begin{array}{r}1 \\
190\end{array}$ & $\begin{array}{c}1 \\
180\end{array}$ & $\begin{array}{c}1 \\
170\end{array}$ & $\begin{array}{c}1 \\
160\end{array}$ & $\begin{array}{c}1 \\
150\end{array}$ & $\begin{array}{r}1 \\
140\end{array}$ & $\begin{array}{c}1 \\
130\end{array}$ & $\begin{array}{c}1 \\
120\end{array}$ & $\begin{array}{r}110 \\
\mathrm{f} 1\end{array}$ & $\begin{array}{l}1 \\
100 \\
\mathrm{pm})\end{array}$ & 90 & $\begin{array}{l}1 \\
80\end{array}$ & $\begin{array}{c}1 \\
70\end{array}$ & $\begin{array}{l}1 \\
60\end{array}$ & $\begin{array}{c}1 \\
50\end{array}$ & $\begin{array}{l}1 \\
40\end{array}$ & $\begin{array}{l}1 \\
30\end{array}$ & $\begin{array}{l}1 \\
20\end{array}$ & $\begin{array}{c}1 \\
10\end{array}$ & $\begin{array}{l}1 \\
0\end{array}$ & $\begin{array}{r}1 \\
-10\end{array}$ \\
\hline
\end{tabular}




\begin{tabular}{|ll|}
\hline \multicolumn{1}{|c|}{ Parameter } & \multicolumn{1}{c|}{ Value } \\
1 Title & youliang-5-296C-P \\
2 Solvent & $\mathrm{CDCl} 3$ \\
3 Temperature & 25.0 \\
4 Relaxation Delay & 10.0000 \\
5 Spectrometer Frequency & 499.86 \\
\hline
\end{tabular}
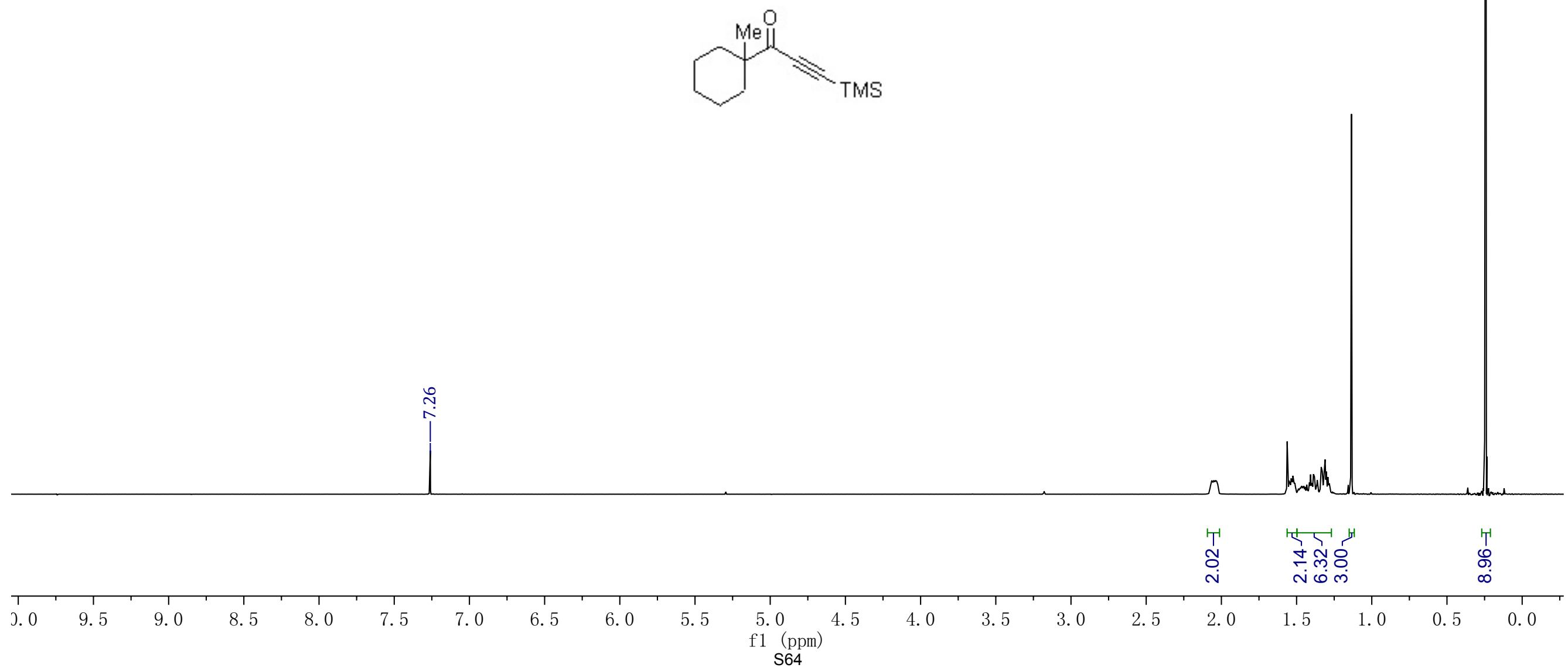


\begin{tabular}{|ll|}
\hline \multicolumn{1}{|c|}{ Parameter } & \multicolumn{1}{c|}{ Value } \\
1 Title & youliang-5-296C-P-C13C \\
2 Solvent & CDC13 \\
3 Temperature & 25.0 \\
4 Relaxation Delay & 1.0000 \\
5 Spectrometer Frequency & 125.70 \\
\hline
\end{tabular}
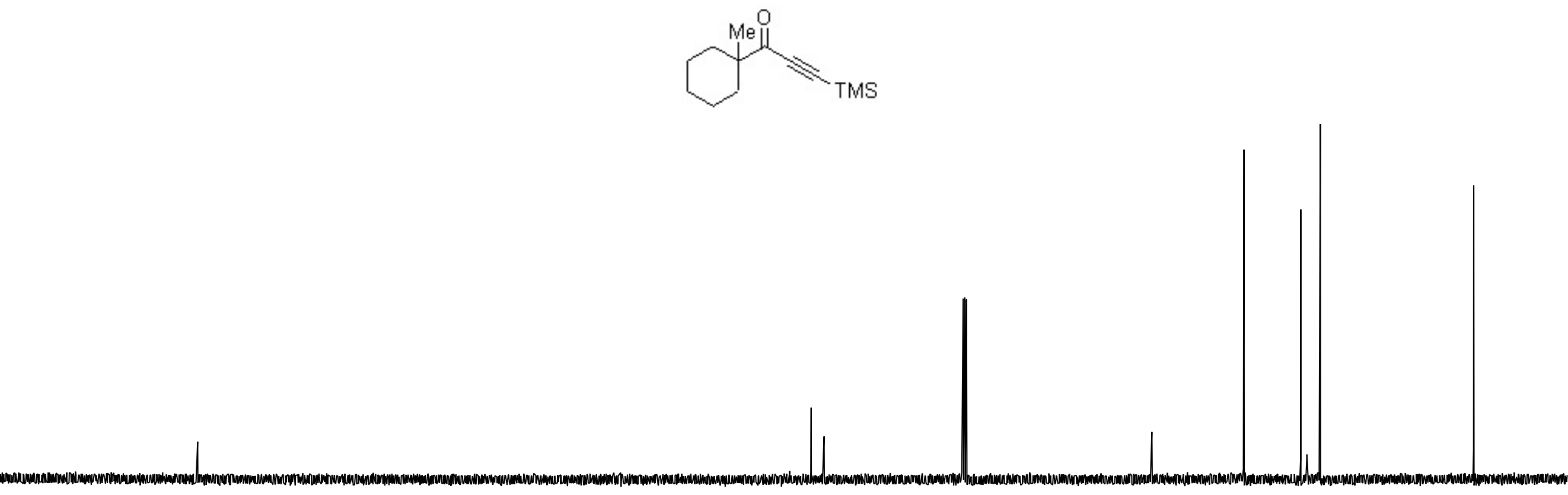

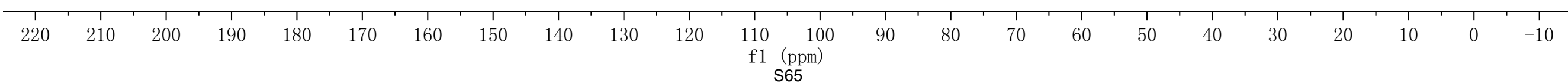




\begin{tabular}{|c|c|}
\hline Parameter & Value \\
\hline 1 Title & youliang-6-12-A-P-again \\
\hline 2 Solvent & $\mathrm{CDCl} 3$ \\
\hline 3 Spectrometer Frequency & 499.86 \\
\hline 4 Nucleus & $1 \mathrm{H}$ \\
\hline
\end{tabular}
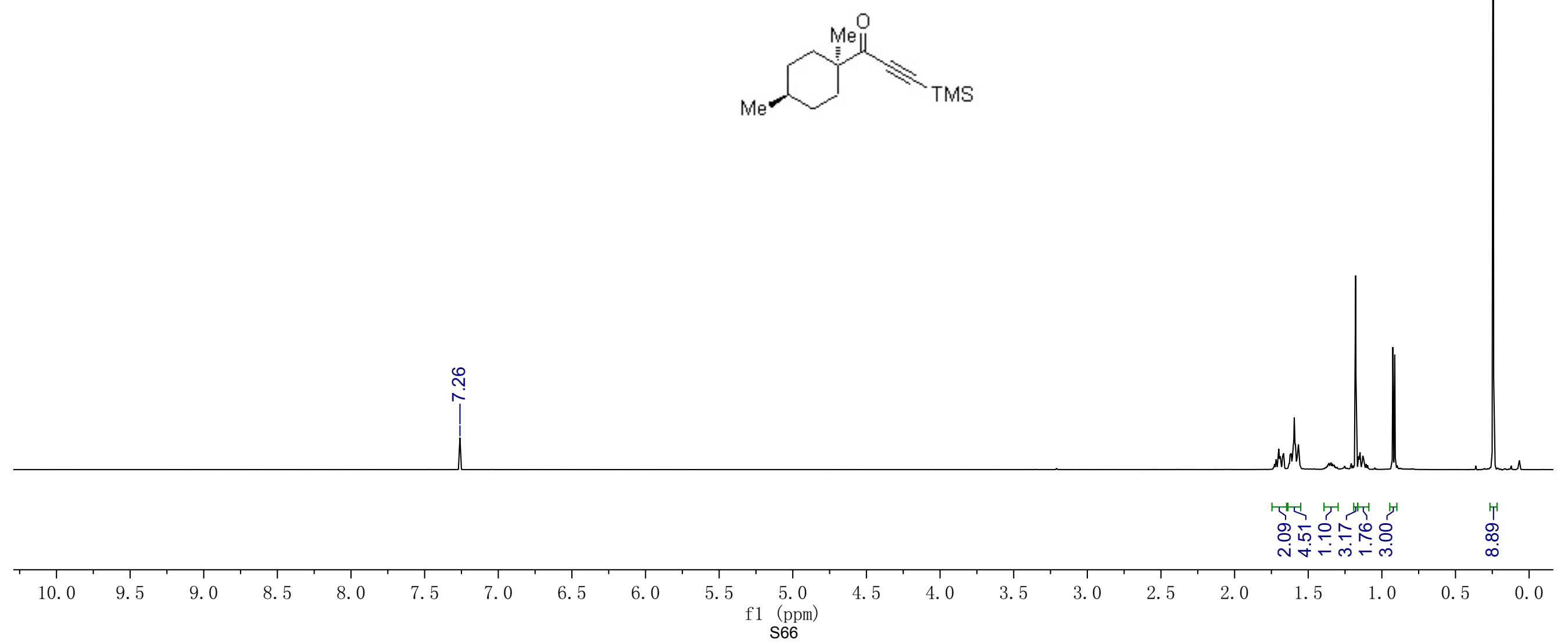
Spectrometer Frequency 125.70

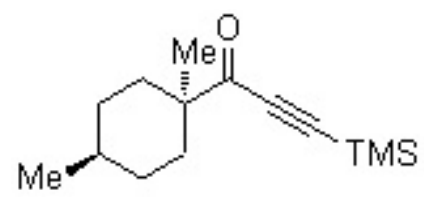

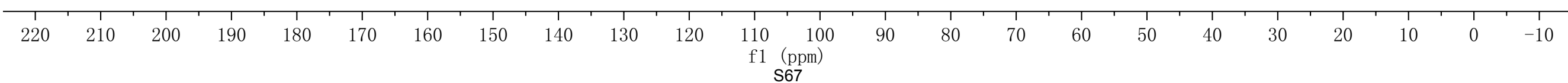




\begin{tabular}{|c|c|}
\hline Parameter & Value \\
\hline 1 Title & youliang-6-8-A-P \\
\hline 2 Solvent & $\mathrm{CDCl} 3$ \\
\hline 3 Spectrometer Frequency & 499.86 \\
\hline 4 Nucleus & $1 \mathrm{H}$ \\
\hline
\end{tabular}
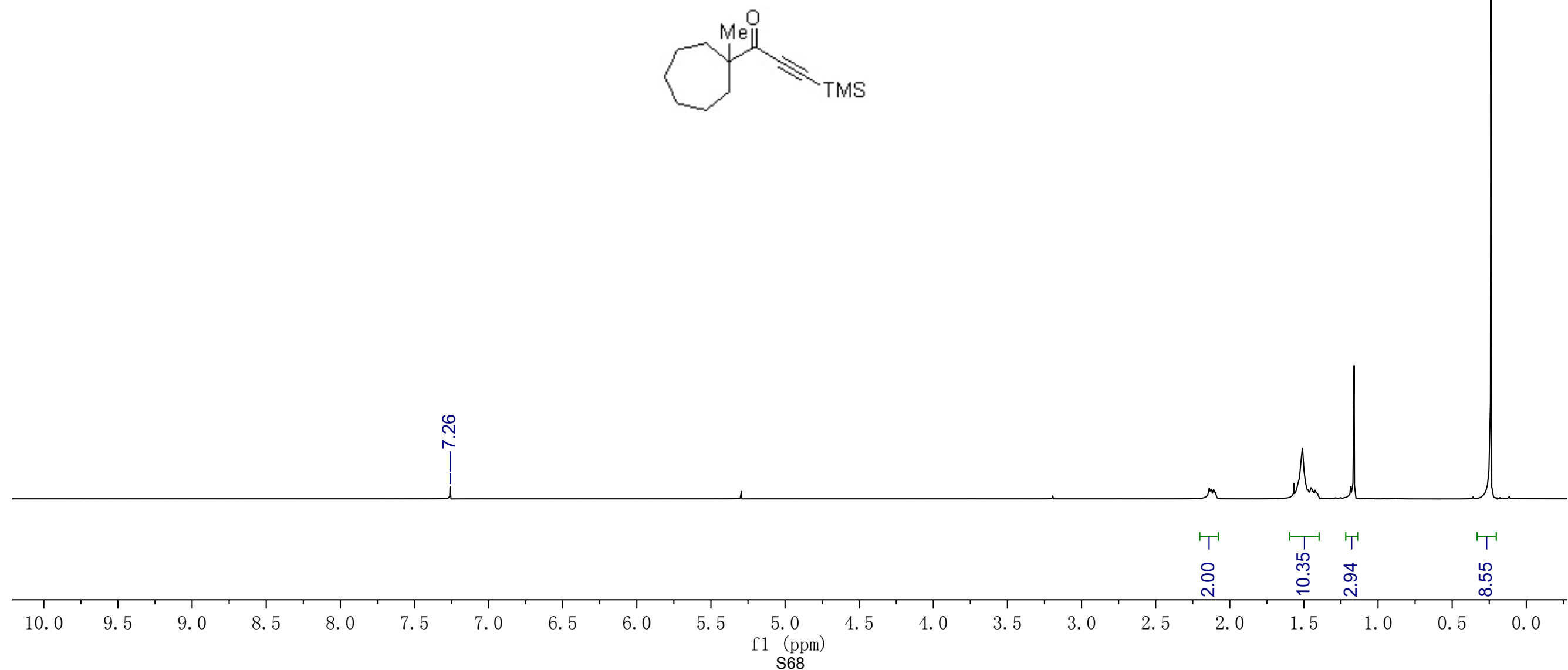


\begin{tabular}{|ll|}
\hline \multicolumn{1}{|c|}{ Parameter } & \multicolumn{1}{c|}{ Value } \\
1 Title & youliang-6-8-A-P-C13g \\
2 Solvent & $\mathrm{CDCl3}$ \\
3 Spectrometer Frequency & 125.70 \\
4 Nucleus & $13 \mathrm{C}$ \\
\hline
\end{tabular}

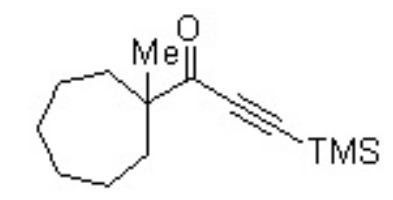

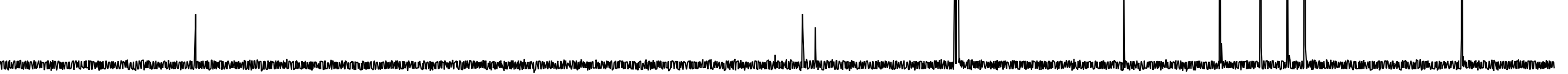

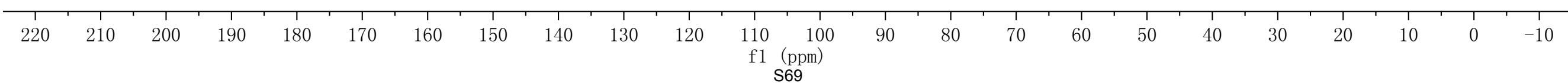




\begin{tabular}{|ll|}
\hline \multicolumn{1}{|c|}{ Parameter } & \multicolumn{1}{c|}{ Value } \\
1 Title & youliang-6-87B-P-major \\
2 Solvent & CDCl3 \\
3 Spectrometer Frequency & 499.86 \\
4 Nucleus & $1 \mathrm{H}$ \\
\hline
\end{tabular}
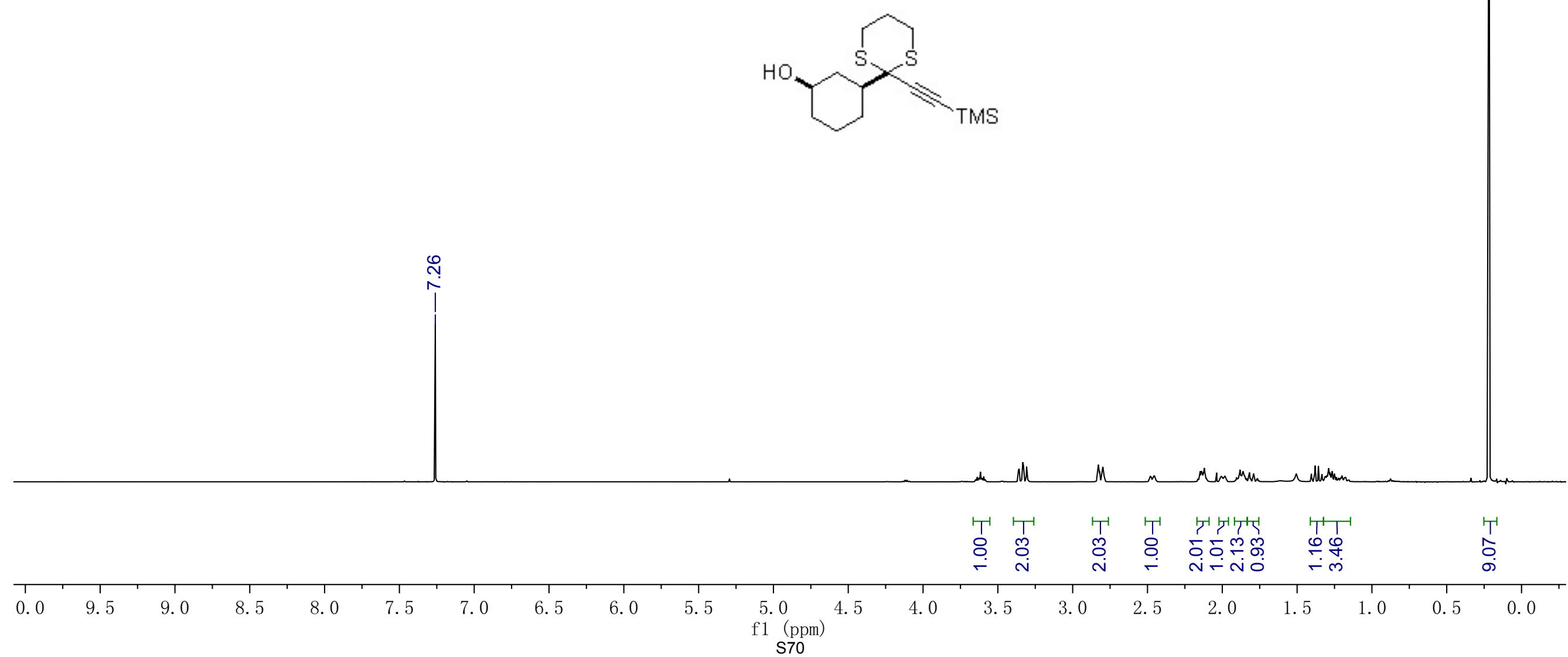
rometer Frequency 125.70
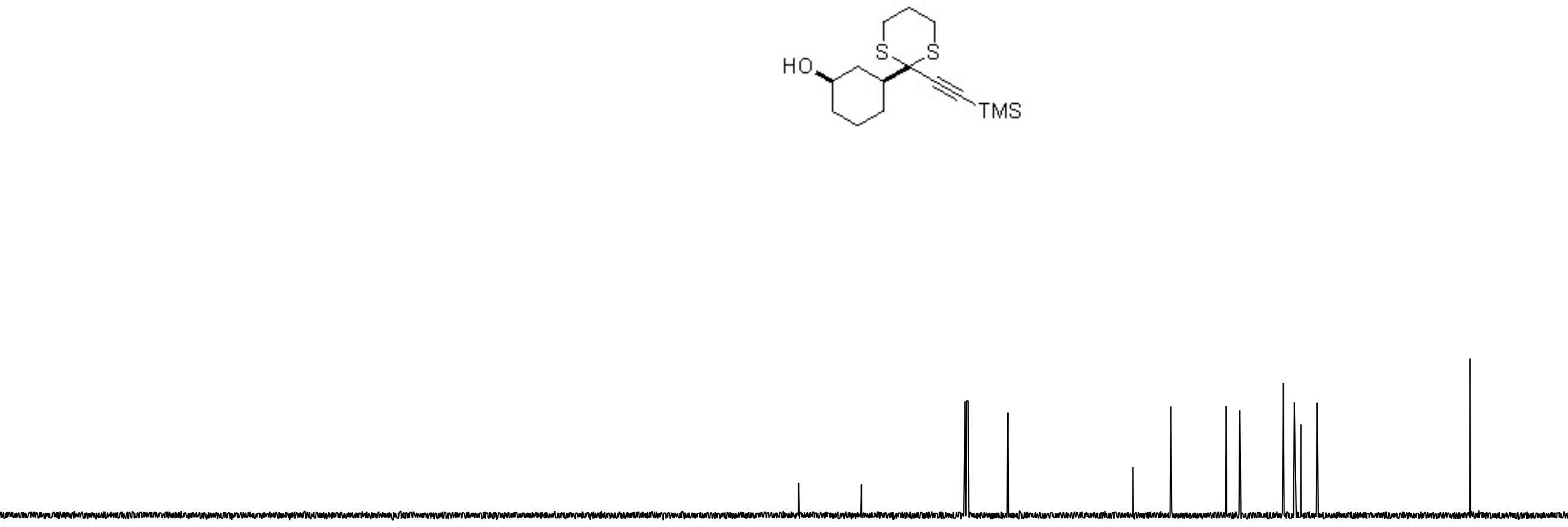

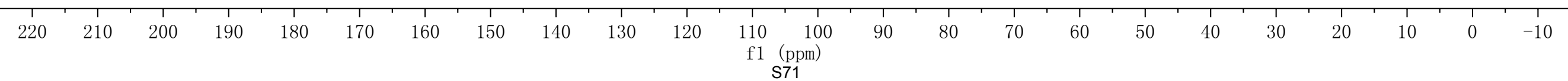



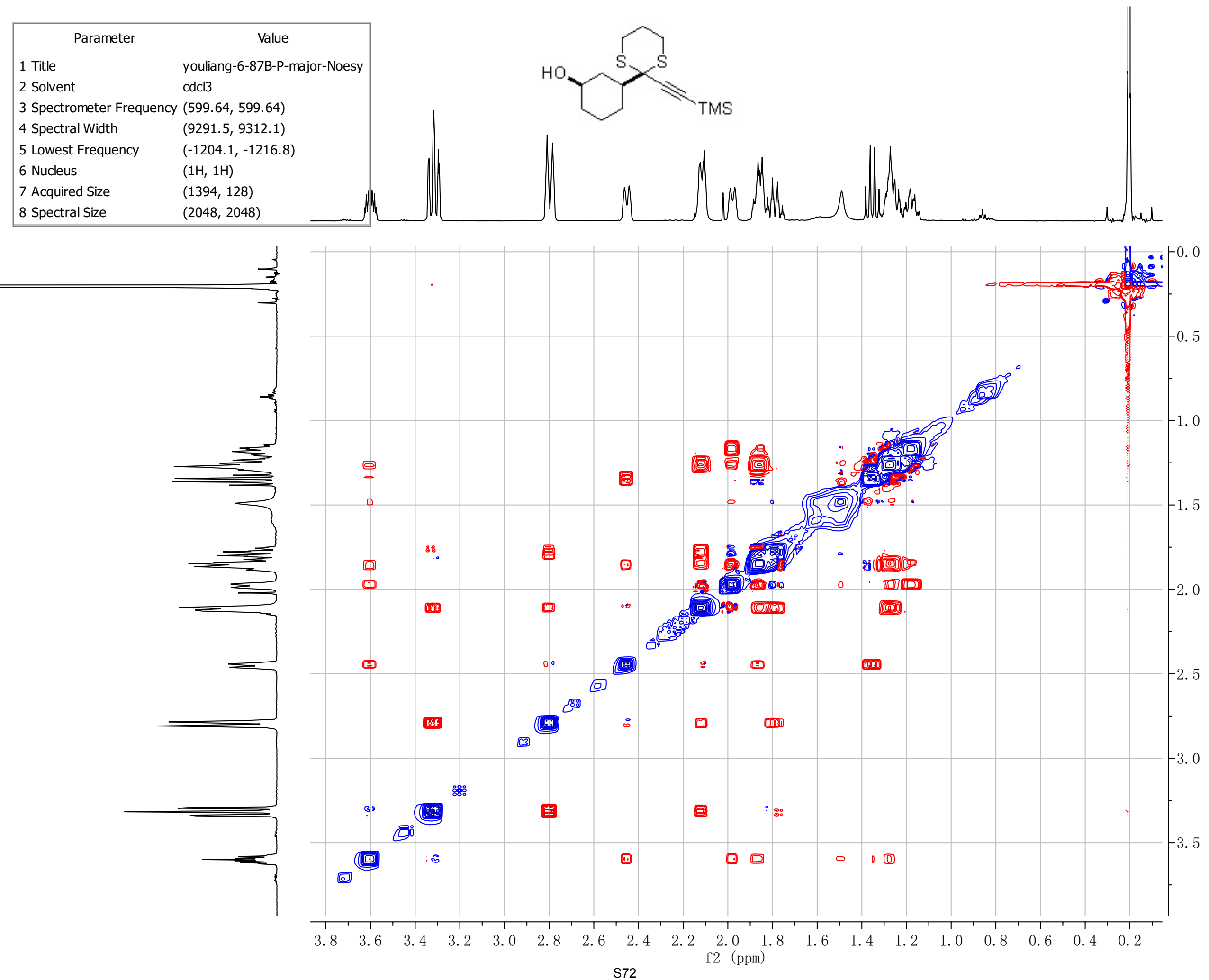


\begin{tabular}{|ll|}
\hline \multicolumn{1}{|c|}{ Parameter } & \multicolumn{1}{c|}{ Value } \\
1 Title & youliang-6-101C-P1 \\
2 Solvent & CDCl3 \\
3 Spectrometer Frequency & 499.86 \\
4 Nucleus & $1 \mathrm{H}$ \\
\hline
\end{tabular}
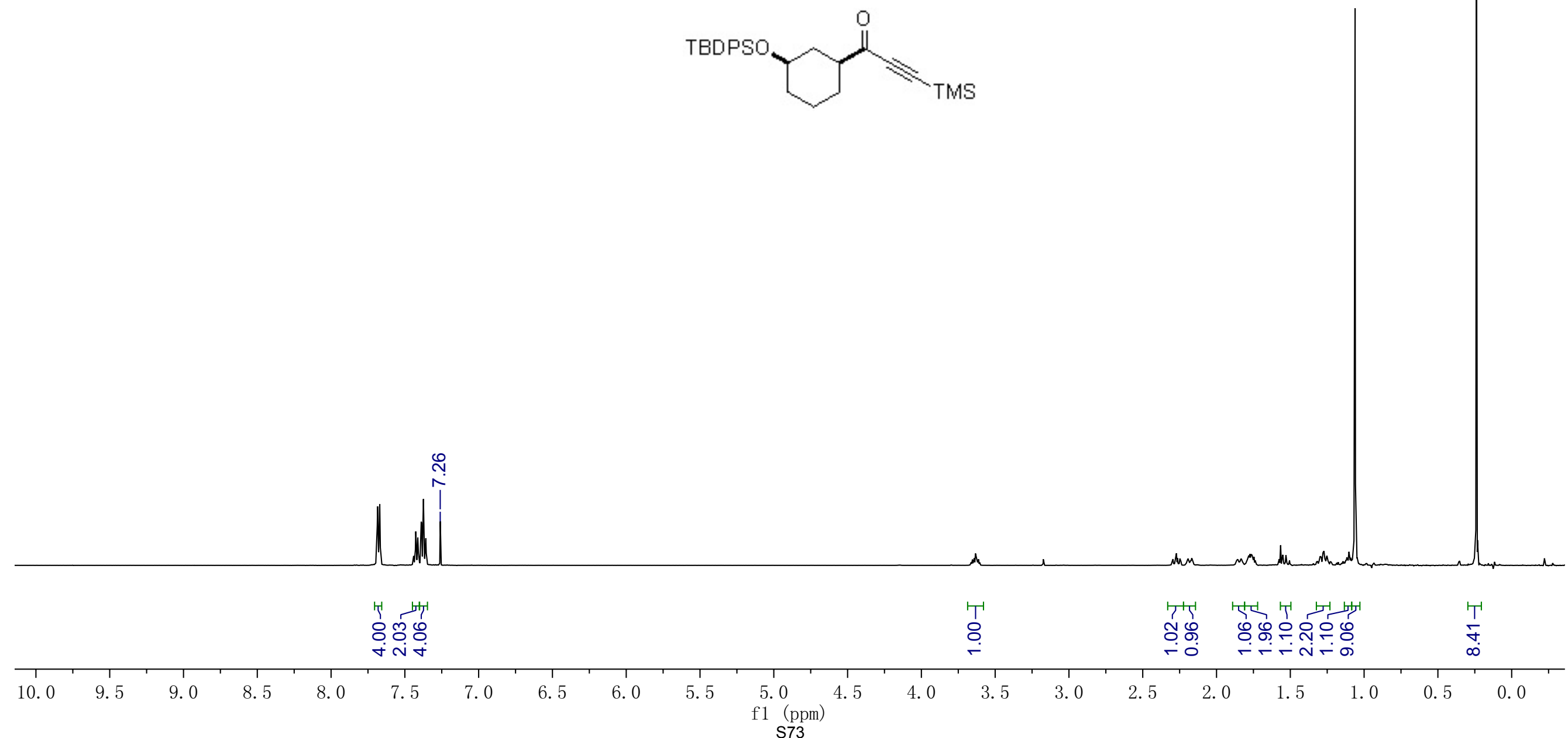
ctrometer Frequency 125.70
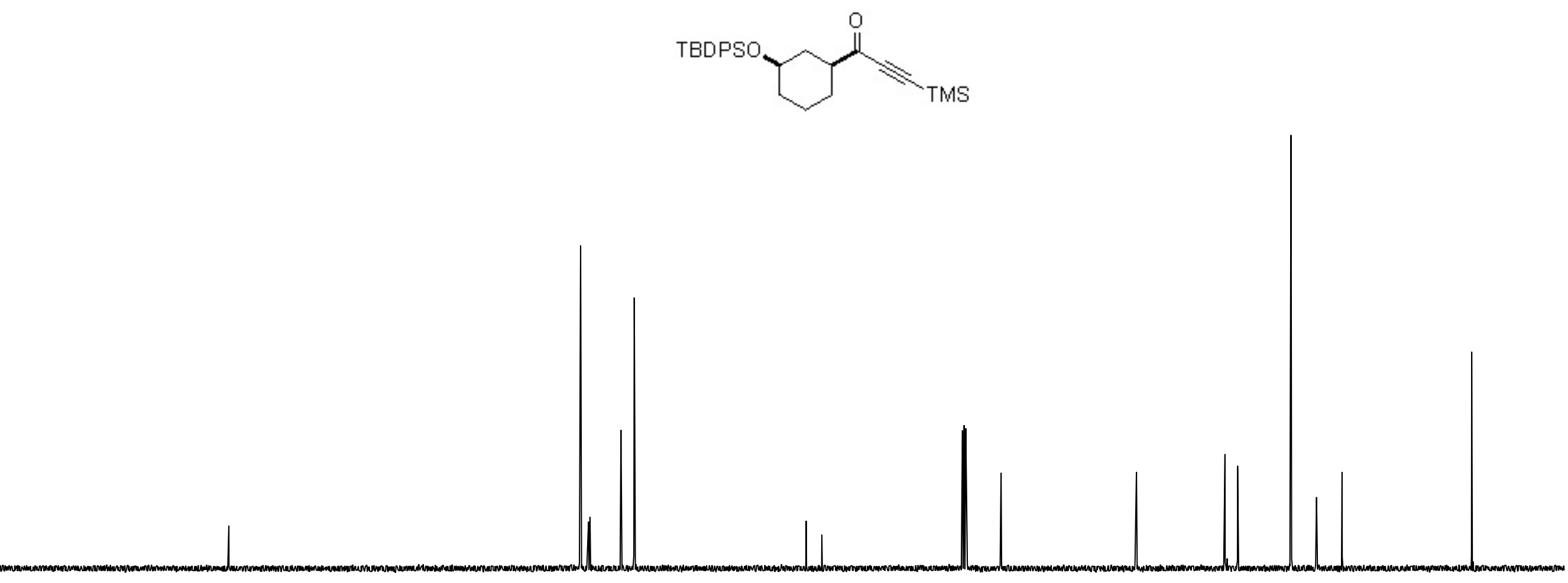

\begin{tabular}{|c|c|c|c|c|c|c|c|c|c|c|c|c|c|c|c|c|c|c|c|c|c|c|c|}
\hline $\begin{array}{c}1 \\
220\end{array}$ & 210 & $\begin{array}{c}1 \\
200\end{array}$ & $\begin{array}{c}1 \\
190\end{array}$ & $\begin{array}{c}1 \\
180\end{array}$ & $\begin{array}{c}1 \\
170\end{array}$ & $\begin{array}{c}1 \\
160\end{array}$ & $\begin{array}{c}1 \\
150\end{array}$ & $\begin{array}{r}1 \\
140\end{array}$ & $\begin{array}{c}1 \\
130\end{array}$ & $\begin{array}{c}1 \\
120\end{array}$ & $\begin{array}{r}110 \\
\mathrm{f} 1\end{array}$ & $\begin{array}{l} \\
100 \\
p m) \\
4\end{array}$ & 9 & $\begin{array}{l}1 \\
80\end{array}$ & $\begin{array}{c}1 \\
70\end{array}$ & $\begin{array}{l}1 \\
60\end{array}$ & $\begin{array}{c}1 \\
50\end{array}$ & $\begin{array}{l}1 \\
40\end{array}$ & $\begin{array}{c}1 \\
30\end{array}$ & $\begin{array}{c}1 \\
20\end{array}$ & $\begin{array}{c}1 \\
10\end{array}$ & $\begin{array}{l}1 \\
0\end{array}$ & -10 \\
\hline
\end{tabular}




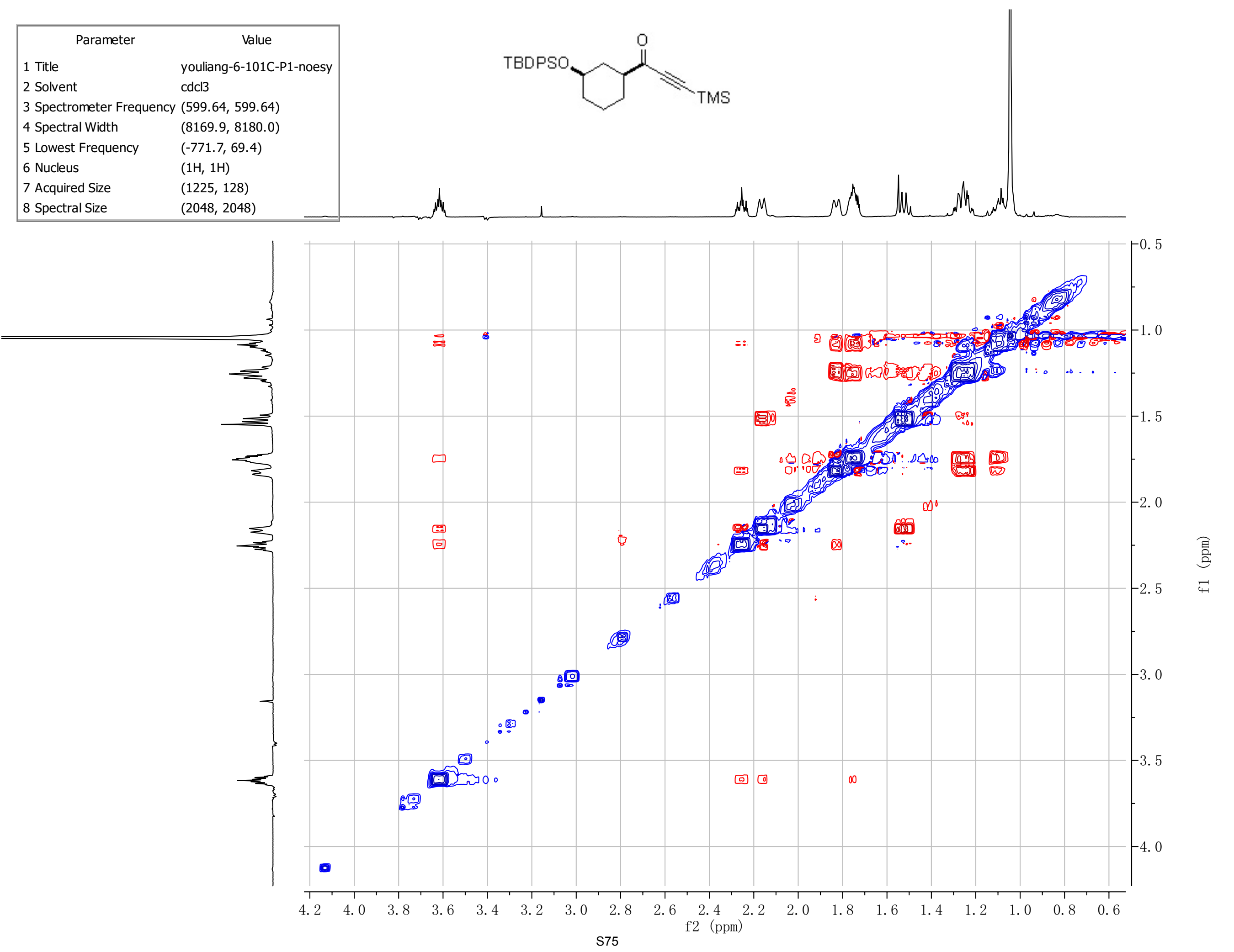




\begin{tabular}{|c|c|}
\hline Parameter & Value \\
\hline 1 Title & youliang-6-87B-P-minor \\
\hline 2 Solvent & $\mathrm{CDCl} 3$ \\
\hline 3 Spectrometer Frequency & 499.86 \\
\hline 4 Nucleus & $1 \mathrm{H}$ \\
\hline
\end{tabular}
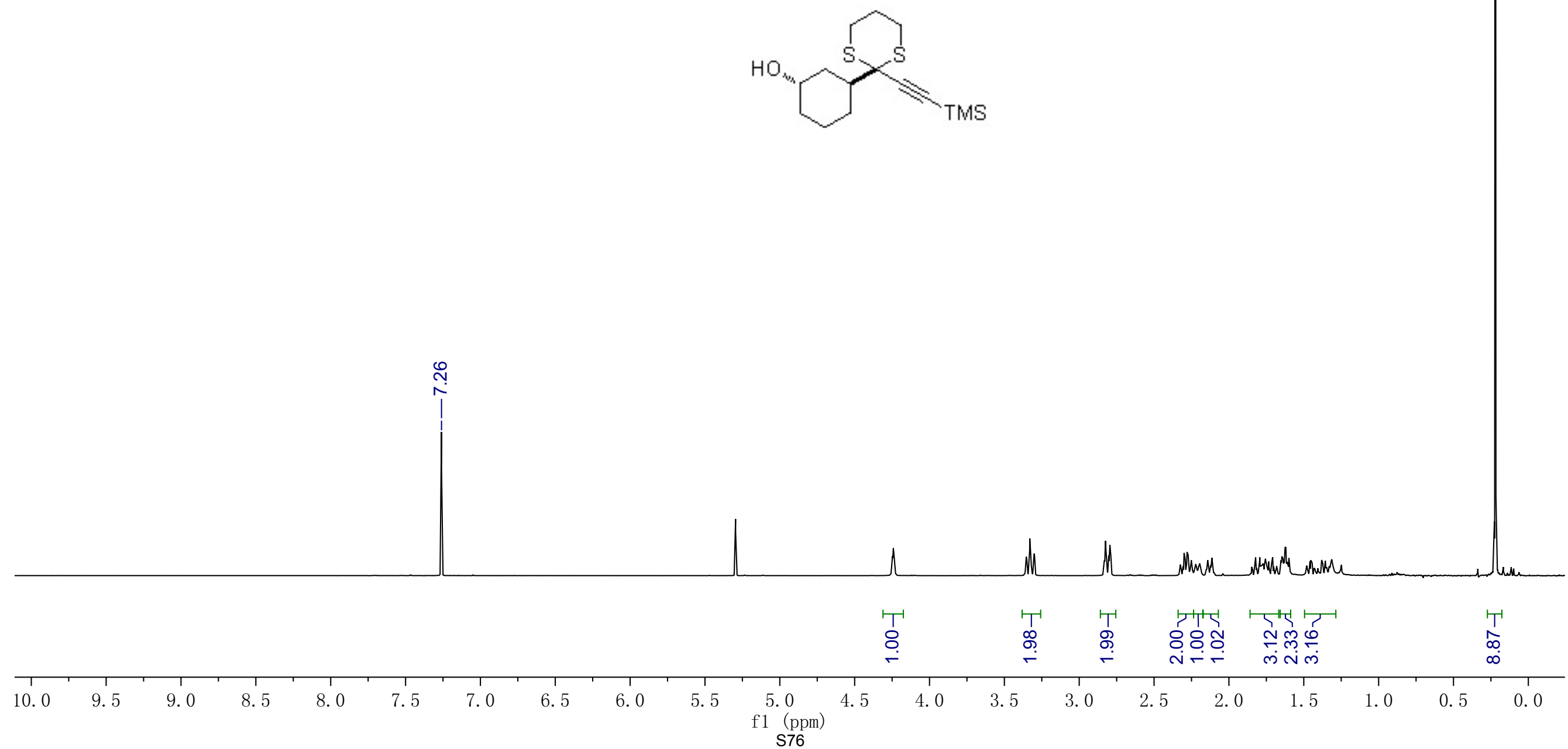
2 Solvent

ouliang-6-87B-P-minor-C13f

Frequency 125.70

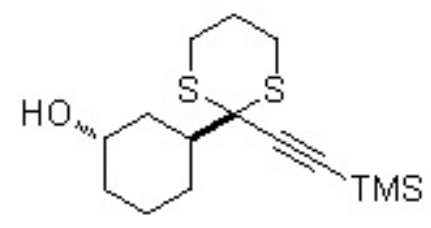

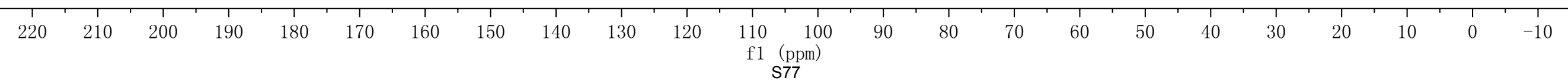




\begin{tabular}{|ll|}
\hline \multicolumn{1}{|c|}{ Parameter } & \multicolumn{1}{c|}{ Value } \\
1 Title & youliang-6-101D-P1 \\
2 Solvent & $\mathrm{CDCl3}$ \\
3 Relaxation Delay & 10.0000 \\
4 Spectrometer Frequency & 499.86 \\
\hline
\end{tabular}
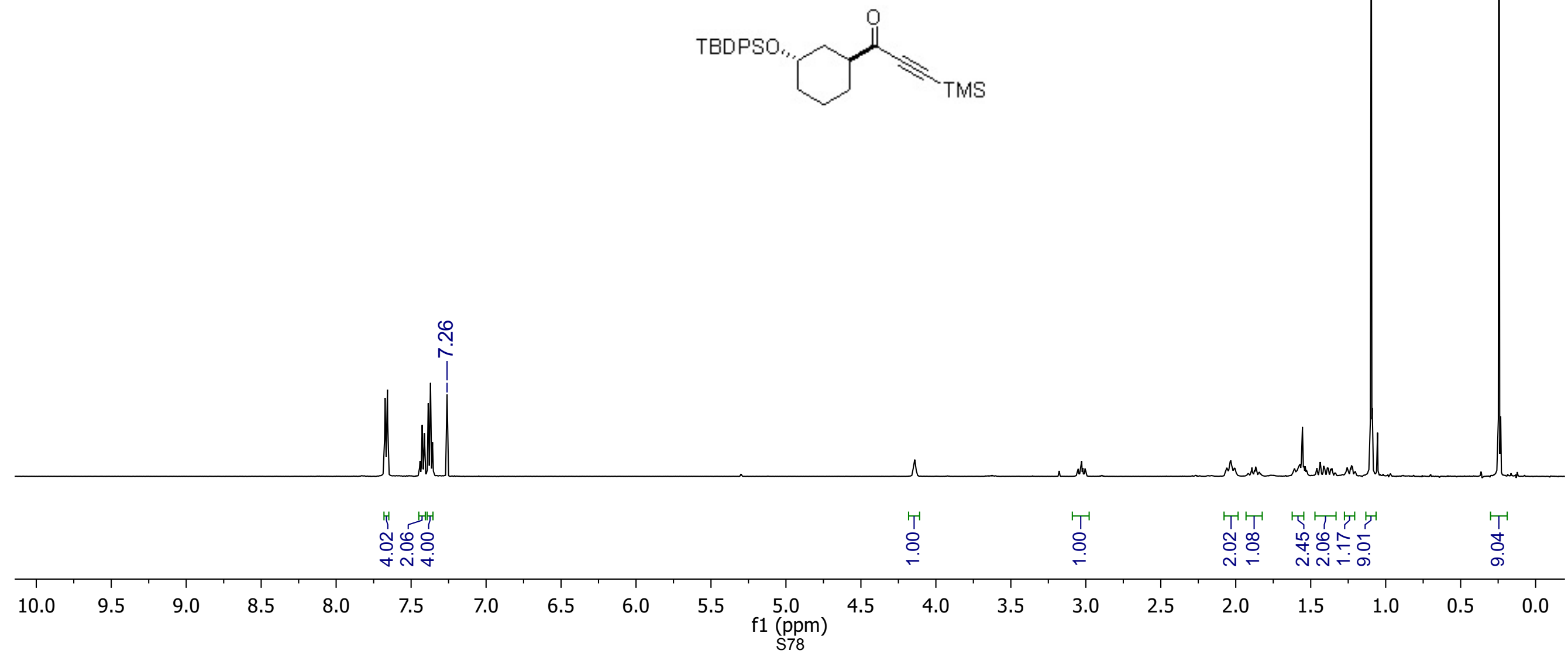


\begin{tabular}{|ll|}
\hline \multicolumn{1}{|c|}{ Parameter } & \multicolumn{1}{c|}{ Value } \\
1 Title & youliang-6-101B-P-C13n \\
2 Solvent & $\mathrm{CDCl} 3$ \\
3 Temperature & 25.0 \\
4 Relaxation Delay & 1.0000 \\
5 Spectrometer Frequency & 125.70 \\
\hline
\end{tabular}
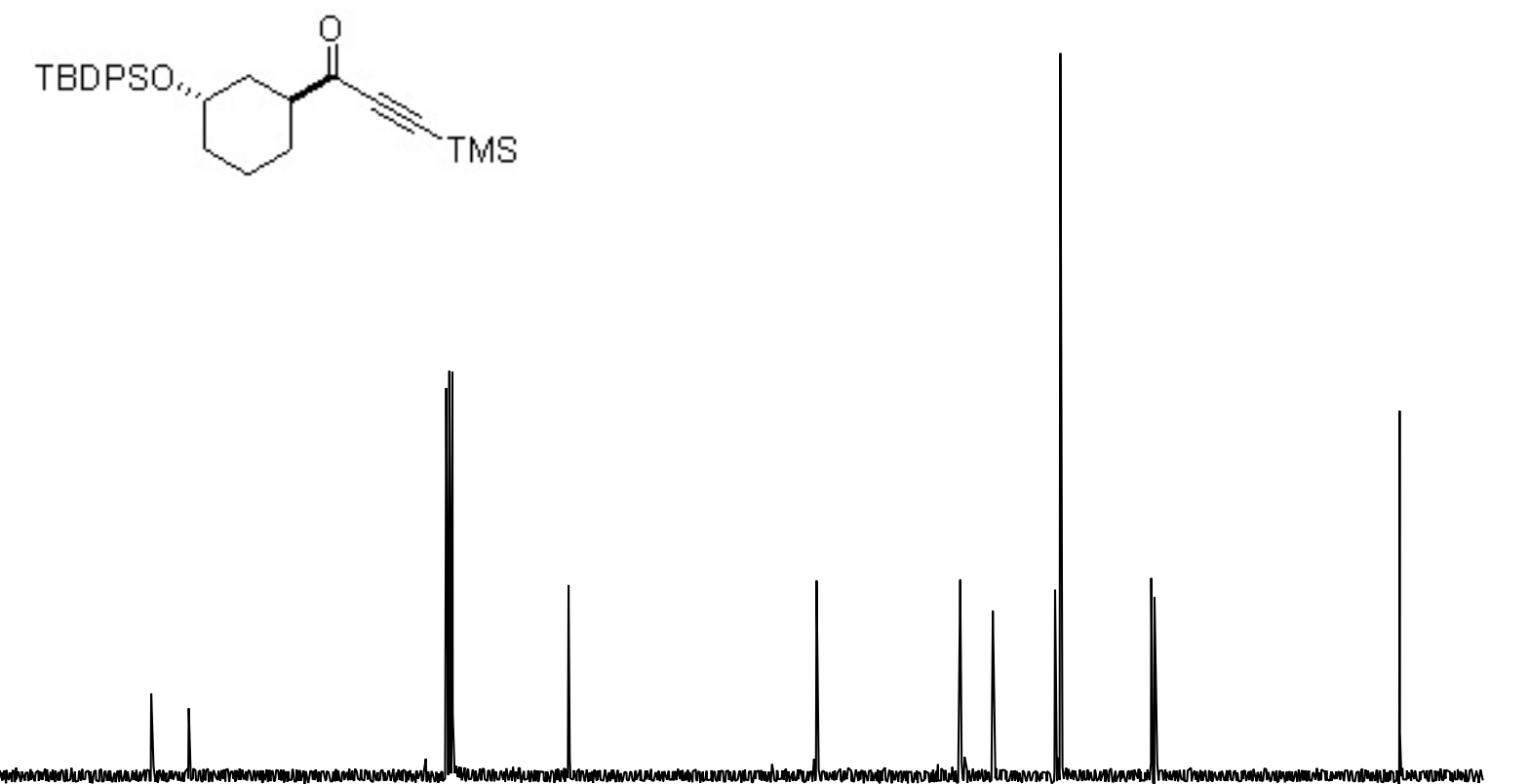

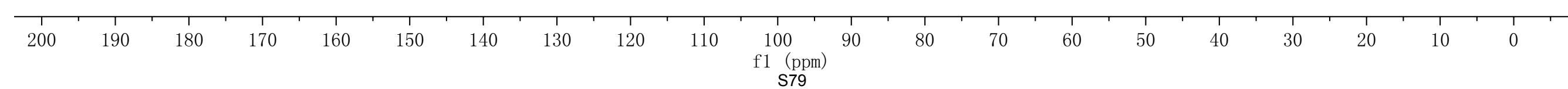




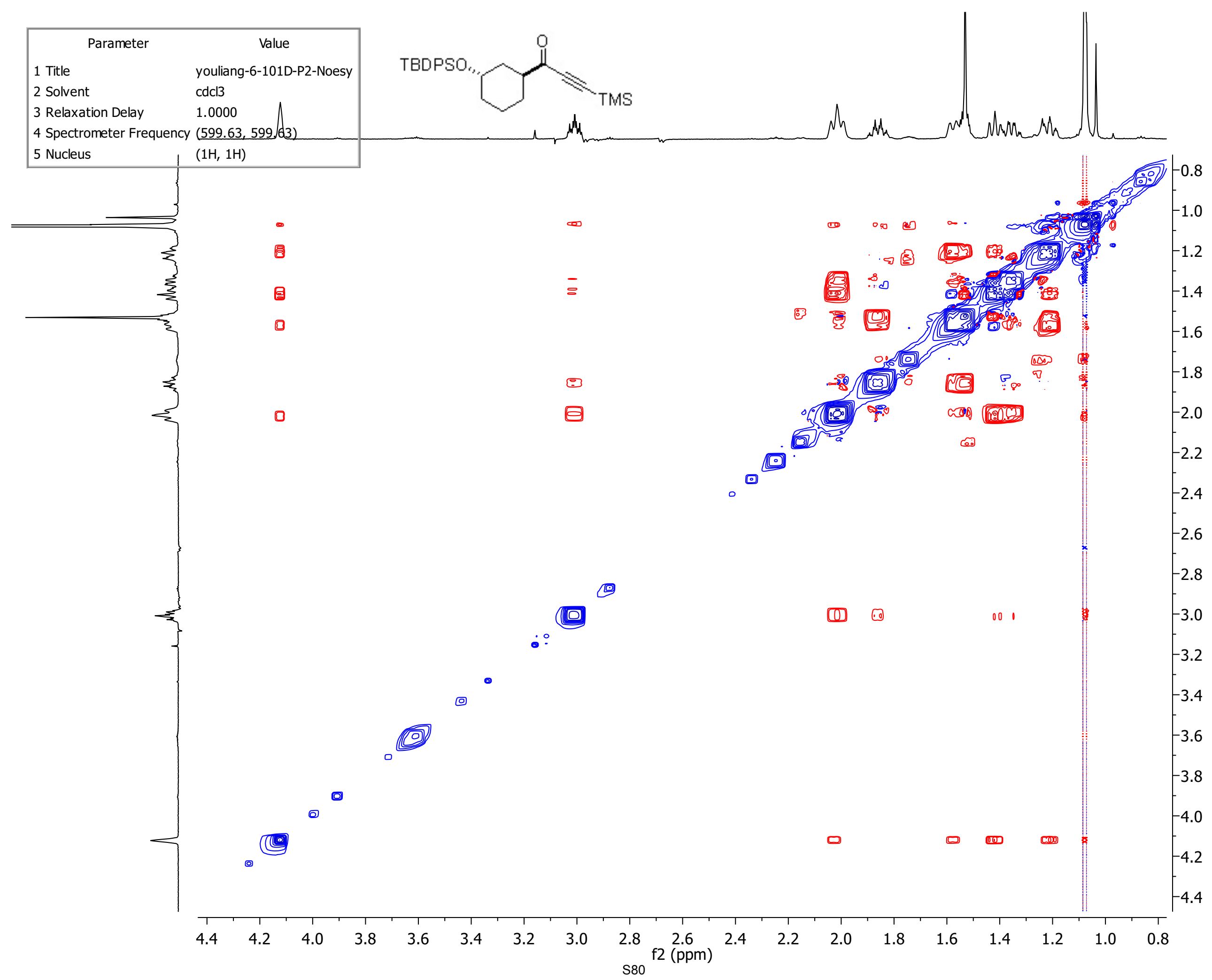




\begin{tabular}{|ll|}
\hline \multicolumn{1}{|c|}{ Parameter } & \multicolumn{1}{c|}{ Value } \\
1 Title & youliang-5-298A-P \\
2 Solvent & $\mathrm{CDCl} 3$ \\
3 Spectrometer Frequency & 499.86 \\
4 Nucleus & $1 \mathrm{H}$ \\
\hline
\end{tabular}

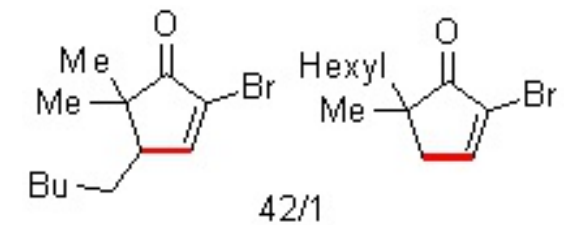

$42 / 1$

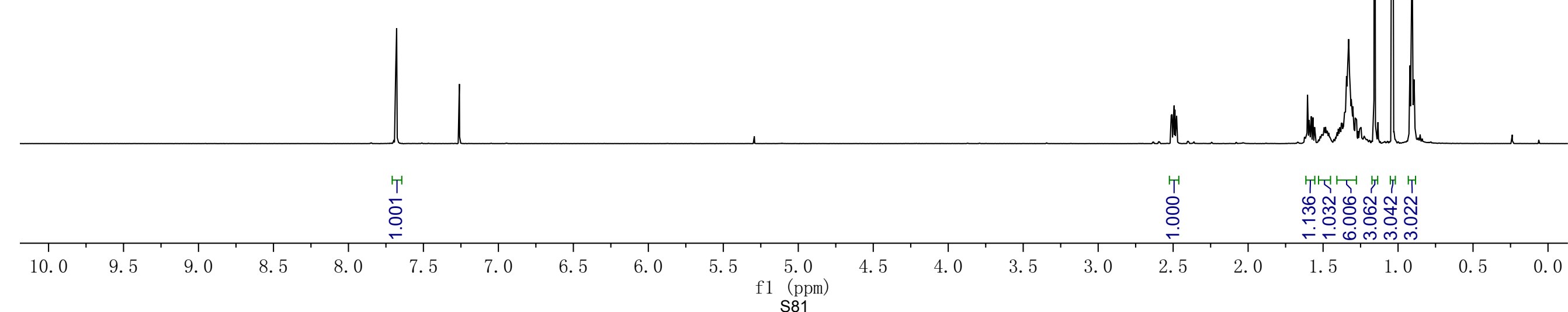




\begin{tabular}{|ll|}
\hline \multicolumn{1}{|c|}{ Parameter } & \multicolumn{1}{c|}{ Value } \\
1 Title & youliang-5-298A-P-C13e \\
2 Solvent & $\mathrm{CDCl} 3$ \\
3 Spectrometer Frequency & 125.70 \\
4 Nucleus & $13 \mathrm{C}$ \\
\hline
\end{tabular}
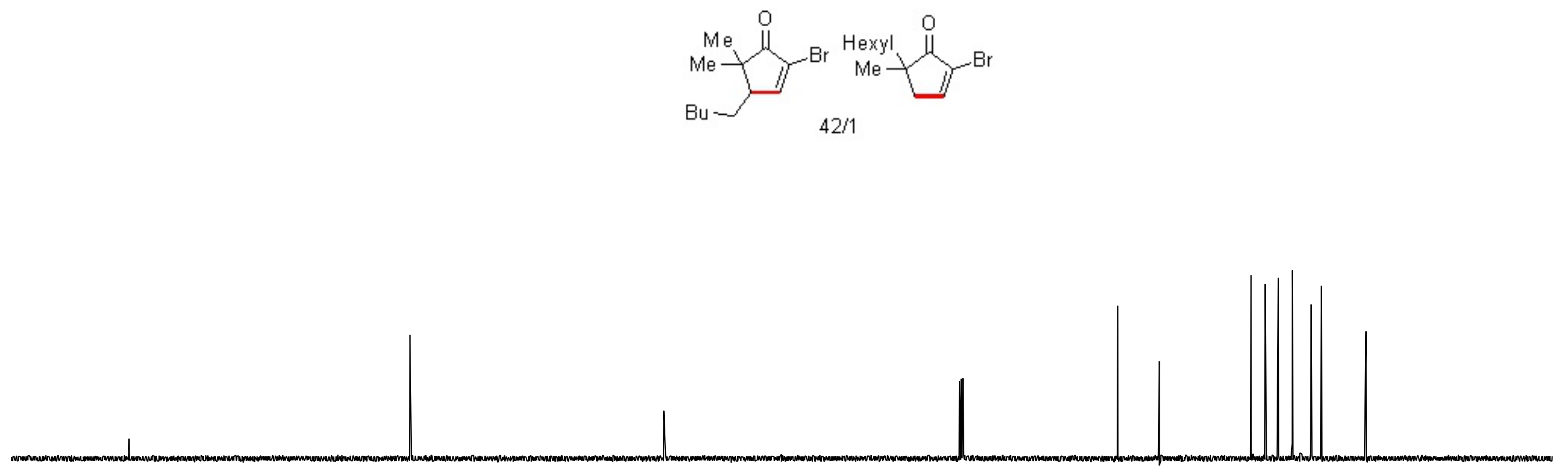

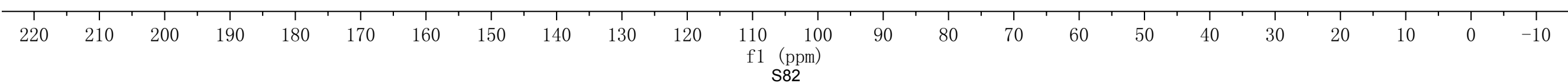




\begin{tabular}{|ll|}
\hline \multicolumn{1}{|c|}{ Parameter } & \multicolumn{1}{c|}{ Value } \\
1 Title & youliang-5-300-B-P \\
2 Solvent & $\mathrm{CDCl} 3$ \\
3 Temperature & 25.0 \\
4 Relaxation Delay & 10.0000 \\
5 Spectrometer Frequency & 499.86 \\
\hline
\end{tabular}
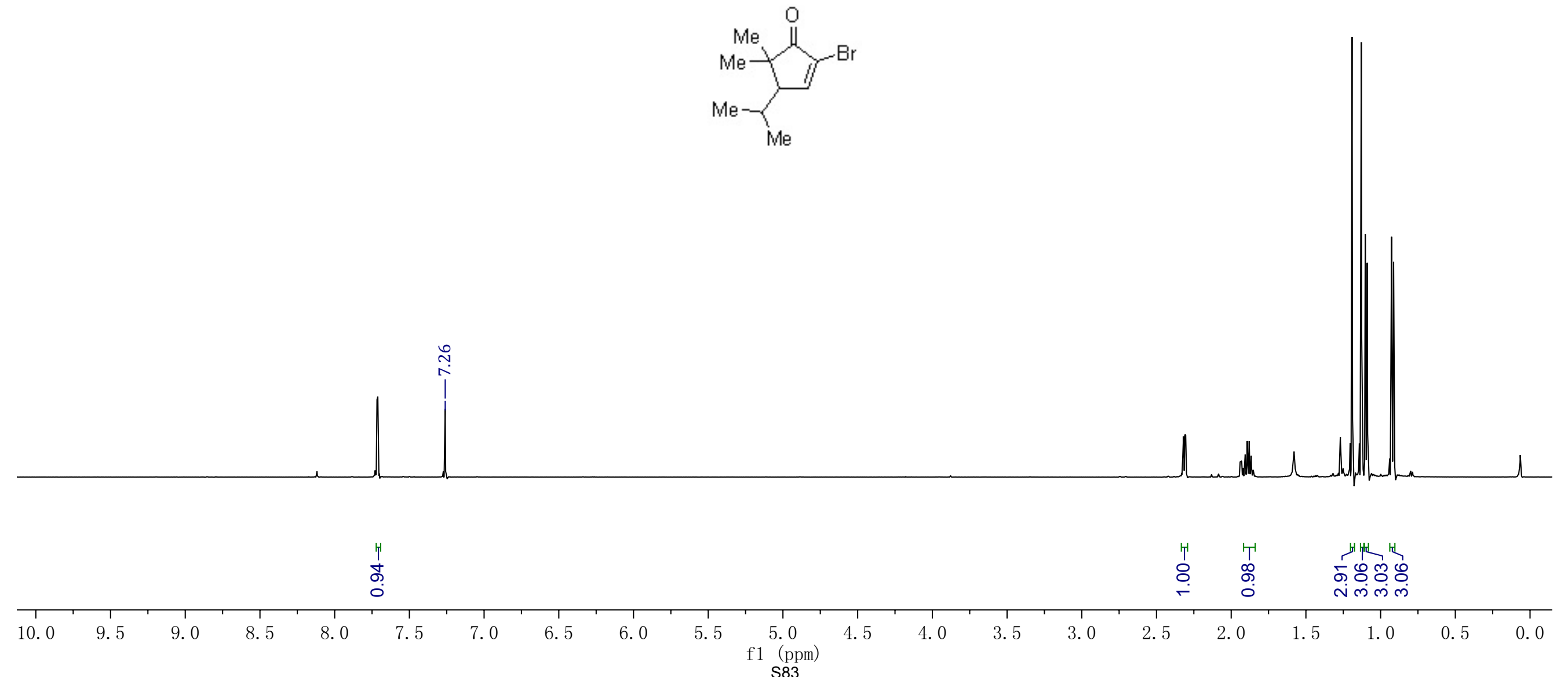


\begin{tabular}{|ll|}
\hline \multicolumn{1}{|c|}{ Parameter } & \multicolumn{1}{c|}{ Value } \\
1 Title & youliang-5-300-B-P-C13f \\
2 Solvent & $\mathrm{CDCl} 3$ \\
3 Temperature & 25.0 \\
4 Relaxation Delay & 1.0000 \\
5 Spectrometer Frequency & 125.70 \\
\hline
\end{tabular}

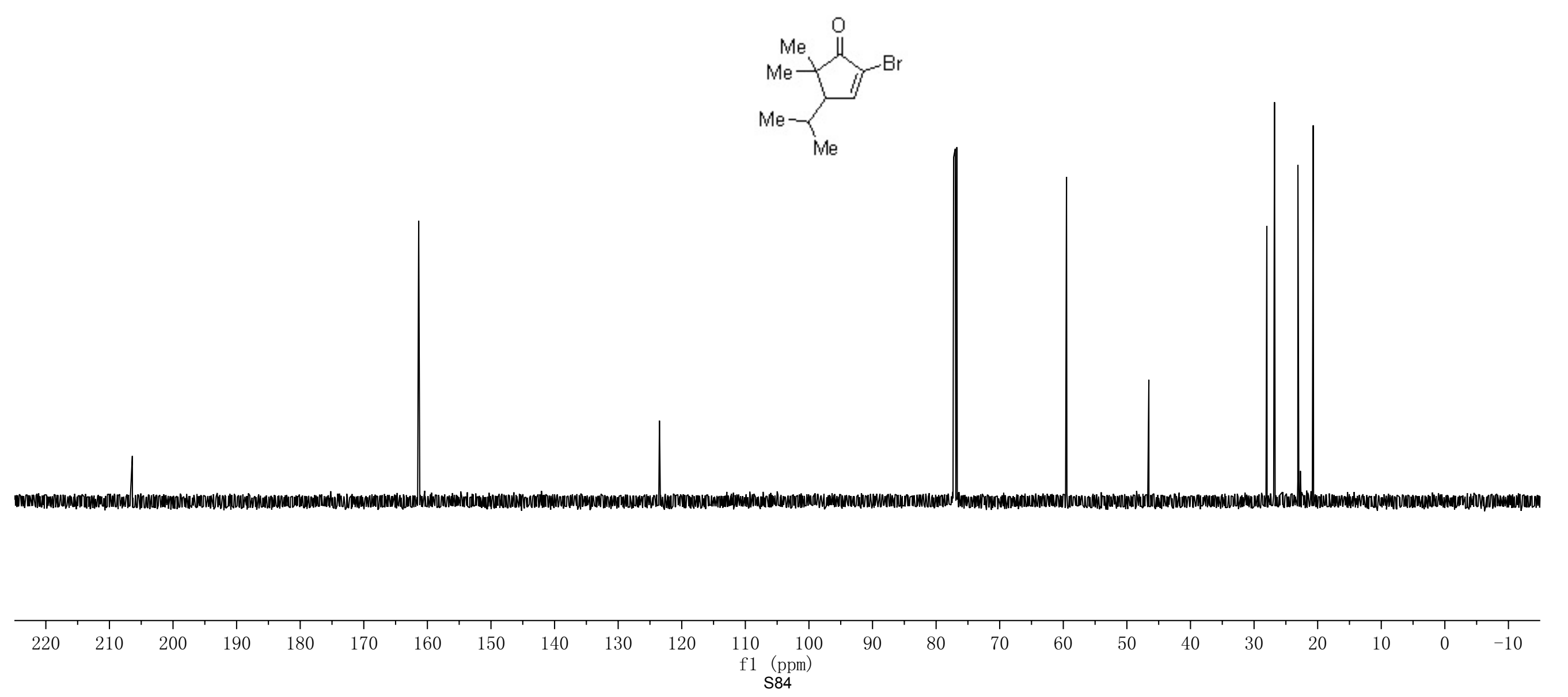




\begin{tabular}{|ll|}
\hline \multicolumn{1}{|c|}{ Parameter } & \multicolumn{1}{c|}{ Value } \\
1 Title & youliang-5-279-B-P-major \\
2 Solvent & CDCl3 \\
3 Temperature & 25.0 \\
4 Relaxation Delay & 10.0000 \\
5 Spectrometer Frequency & 499.86 \\
\hline
\end{tabular}
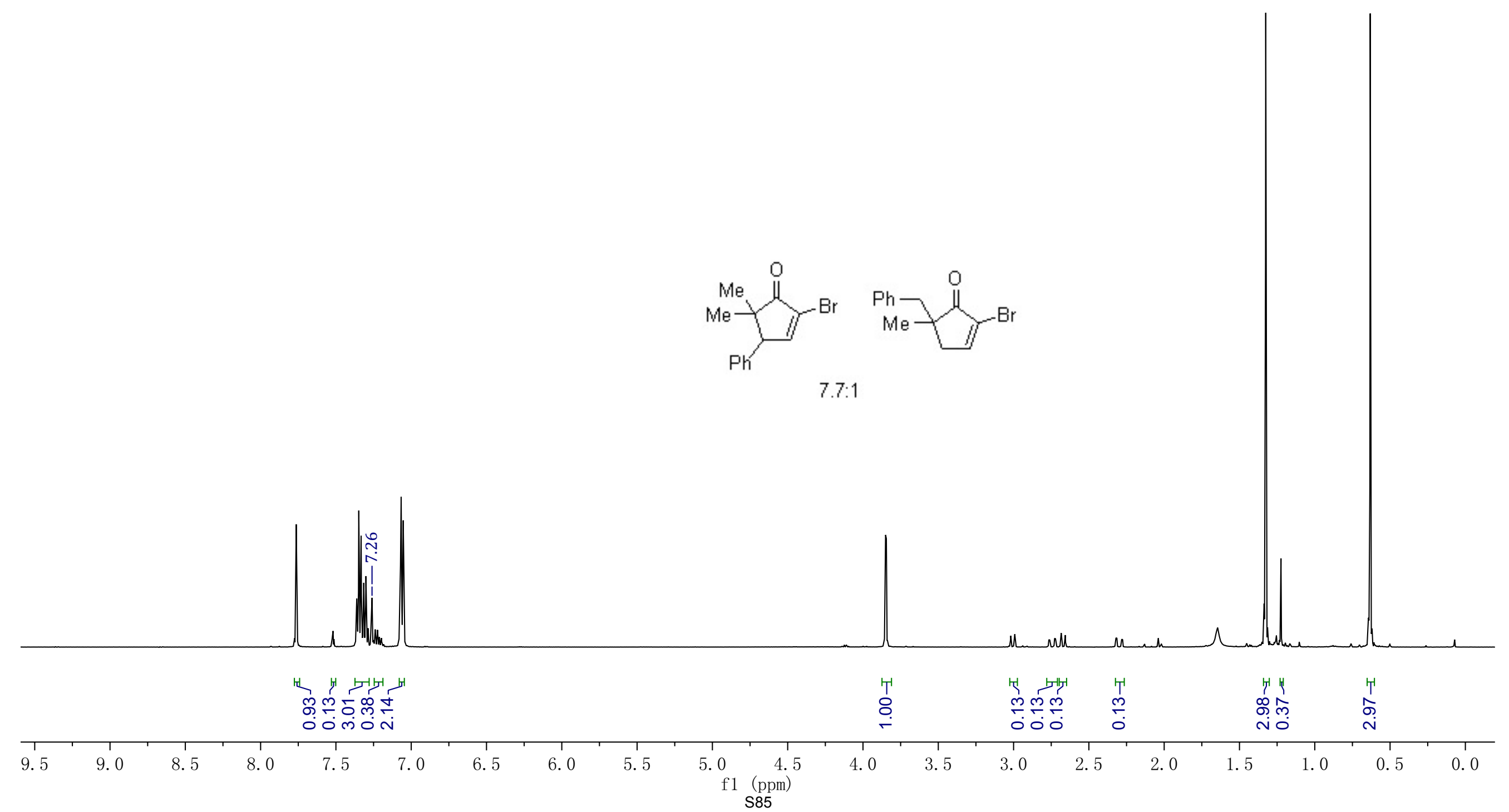


\begin{tabular}{|ll|}
\hline \multicolumn{1}{|c|}{ Parameter } & \multicolumn{1}{c|}{ Value } \\
1 Title & youliang-5-279-B-P-major-C13e \\
2 Solvent & $\mathrm{CDCl3}$ \\
3 Temperature & 25.0 \\
4 Relaxation Delay & 1.0000 \\
5 Spectrometer Frequency & 125.70 \\
\hline
\end{tabular}

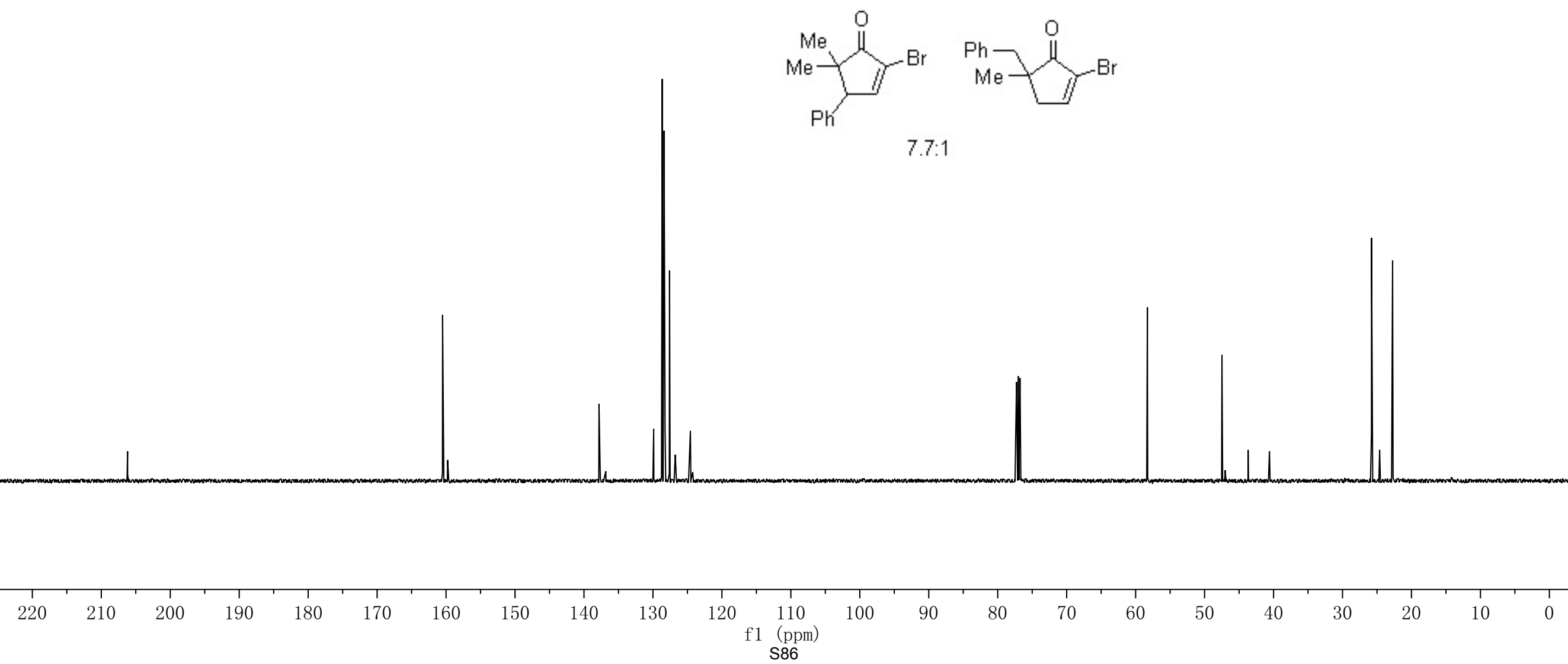




\begin{tabular}{|c|c|}
\hline Parameter & Value \\
\hline 1 Title & youliang-5-279-B-P-SP \\
\hline 2 Solvent & $\mathrm{CDCl} 3$ \\
\hline 3 Spectrometer Frequency & 499.86 \\
\hline 4 Nucleus & $1 \mathrm{H}$ \\
\hline
\end{tabular}
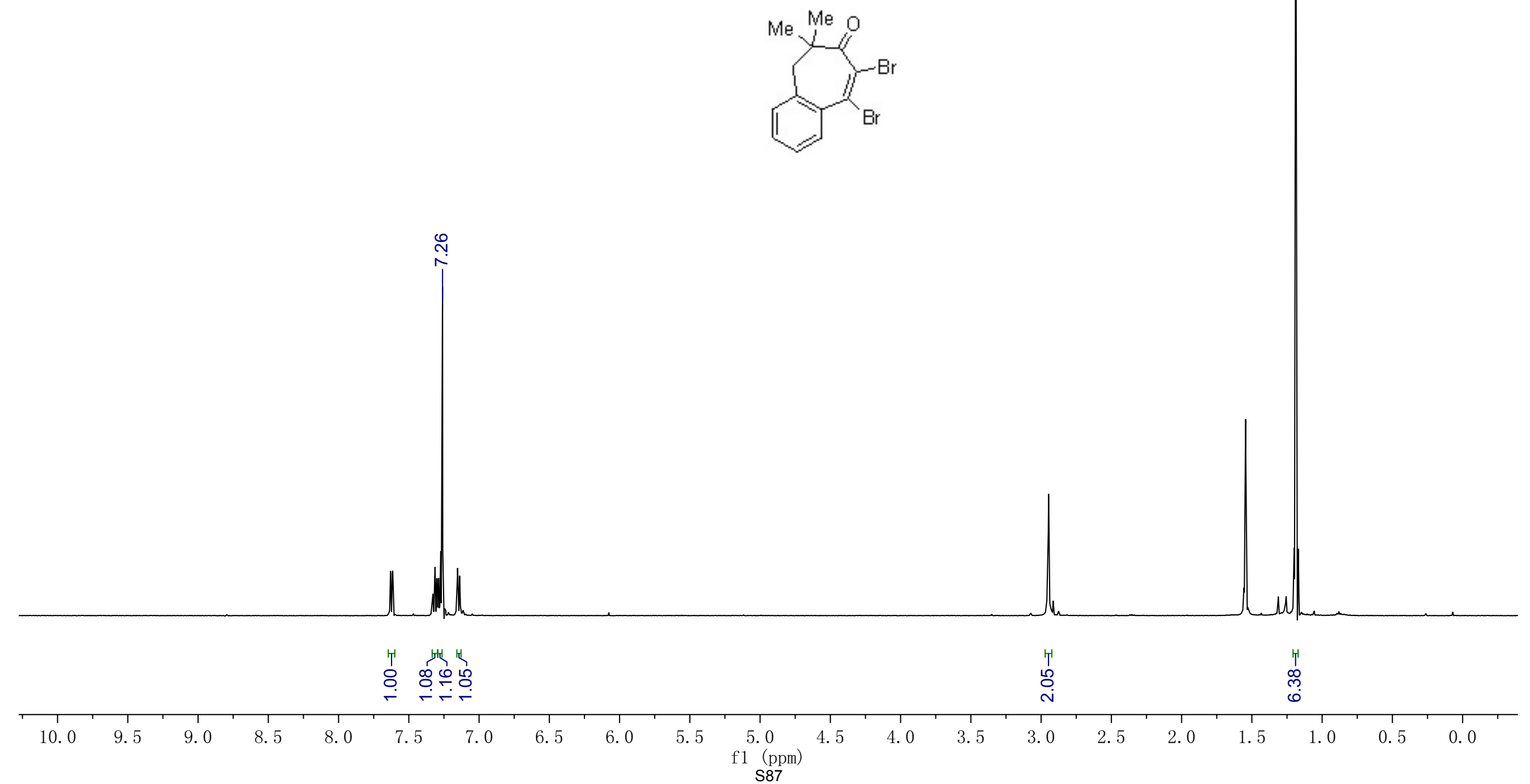


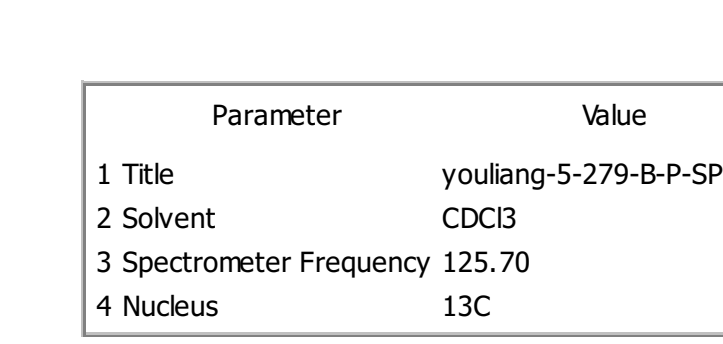

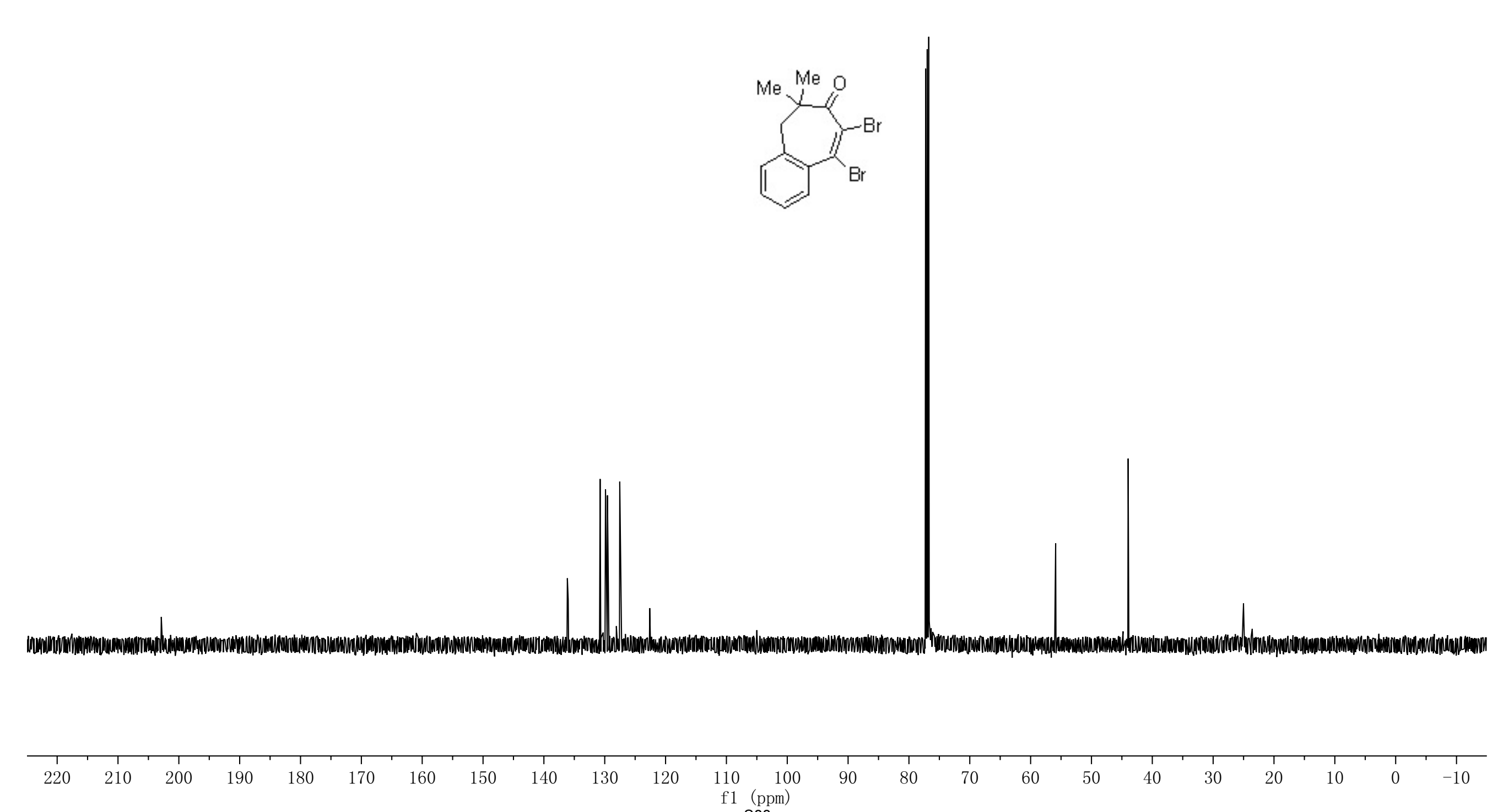




\begin{tabular}{|ll|}
\hline \multicolumn{1}{|c|}{ Parameter } & \multicolumn{1}{c|}{ Value } \\
1 Title & youliang-5-229C-P \\
2 Solvent & $\mathrm{CDCl3}$ \\
3 Spectrometer Frequency & 499.86 \\
4 Nucleus & $1 \mathrm{H}$ \\
\hline
\end{tabular}
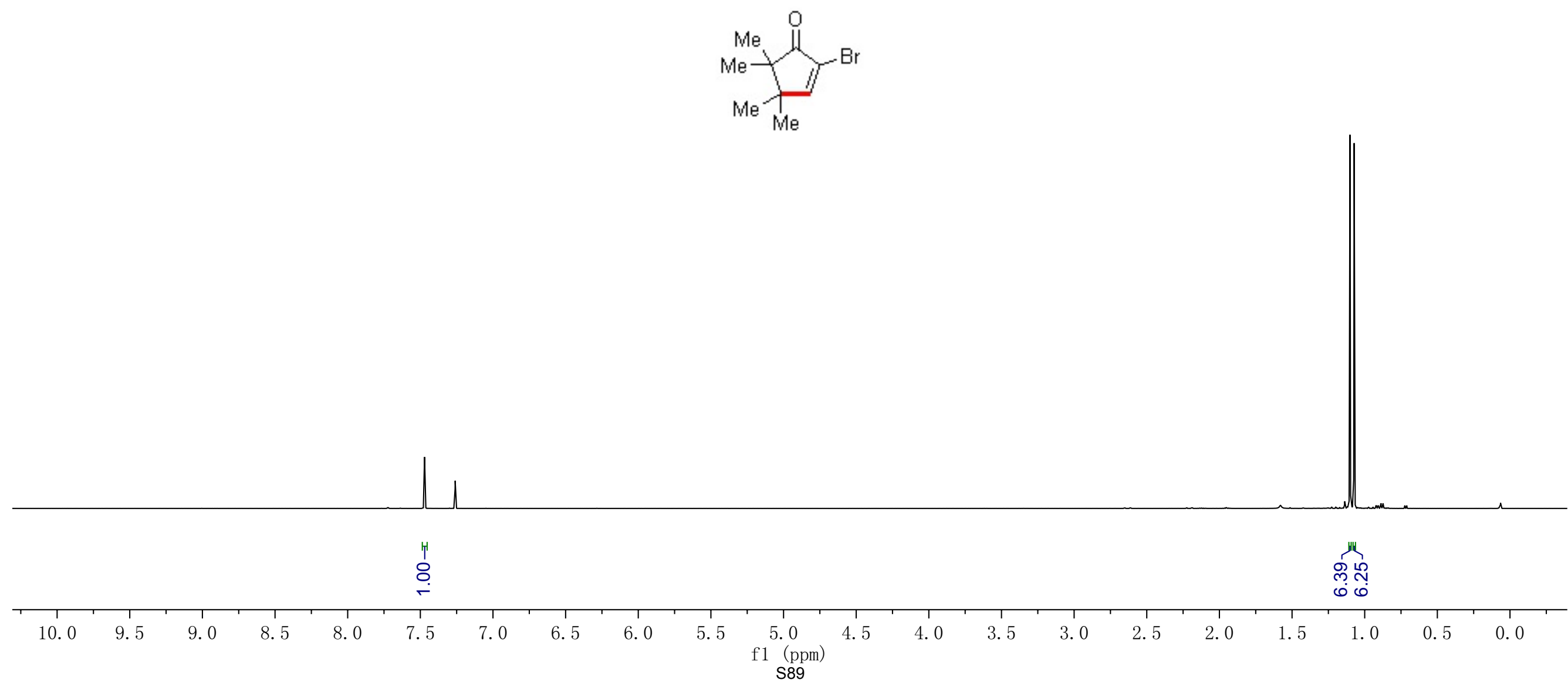


\begin{tabular}{|ll|}
\hline \multicolumn{1}{|c|}{ Parameter } & \multicolumn{1}{c|}{ Value } \\
1 Title & youliang-5-229C-P-C13i \\
2 Solvent & $\mathrm{CDCl} 3$ \\
3 Spectrometer Frequency & 125.70 \\
4 Nucleus & $13 \mathrm{C}$ \\
\hline
\end{tabular}
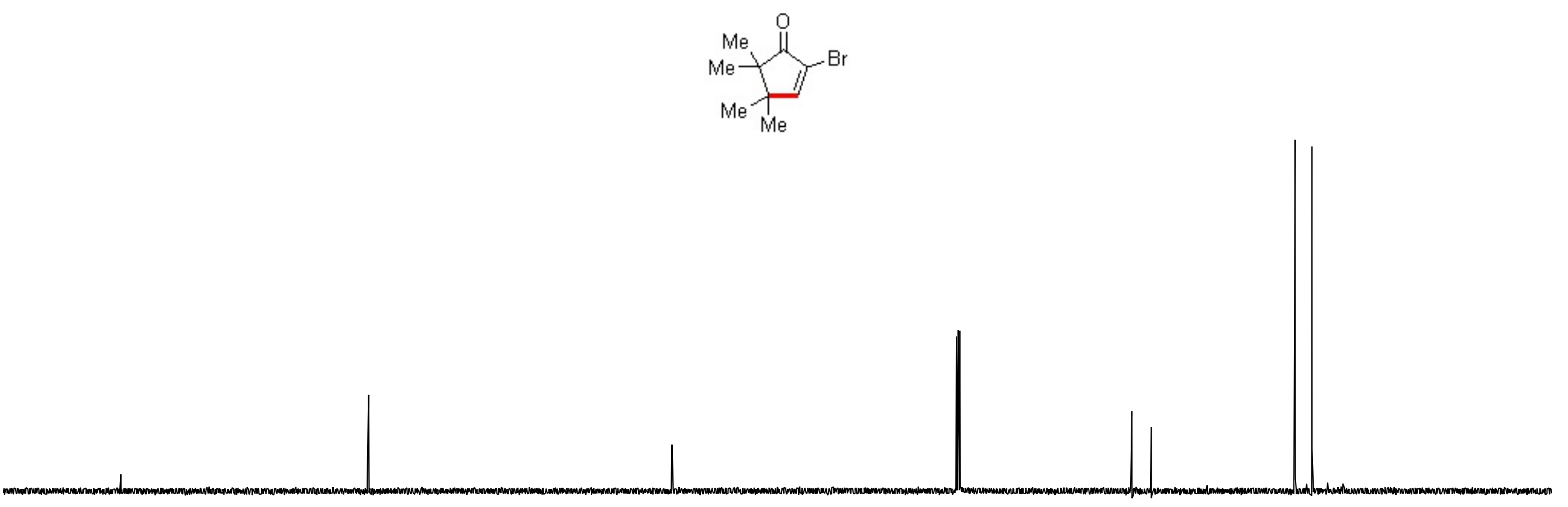

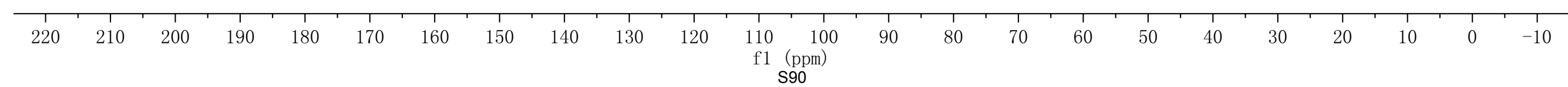




\begin{tabular}{|c|c|}
\hline Parameter & Value \\
\hline 1 Title & youliang-5-298B-P-again \\
\hline 2 Solvent & $\mathrm{CDCl} 3$ \\
\hline 3 Spectrometer Frequency & 499.86 \\
\hline 4 Nucleus & $1 \mathrm{H}$ \\
\hline
\end{tabular}
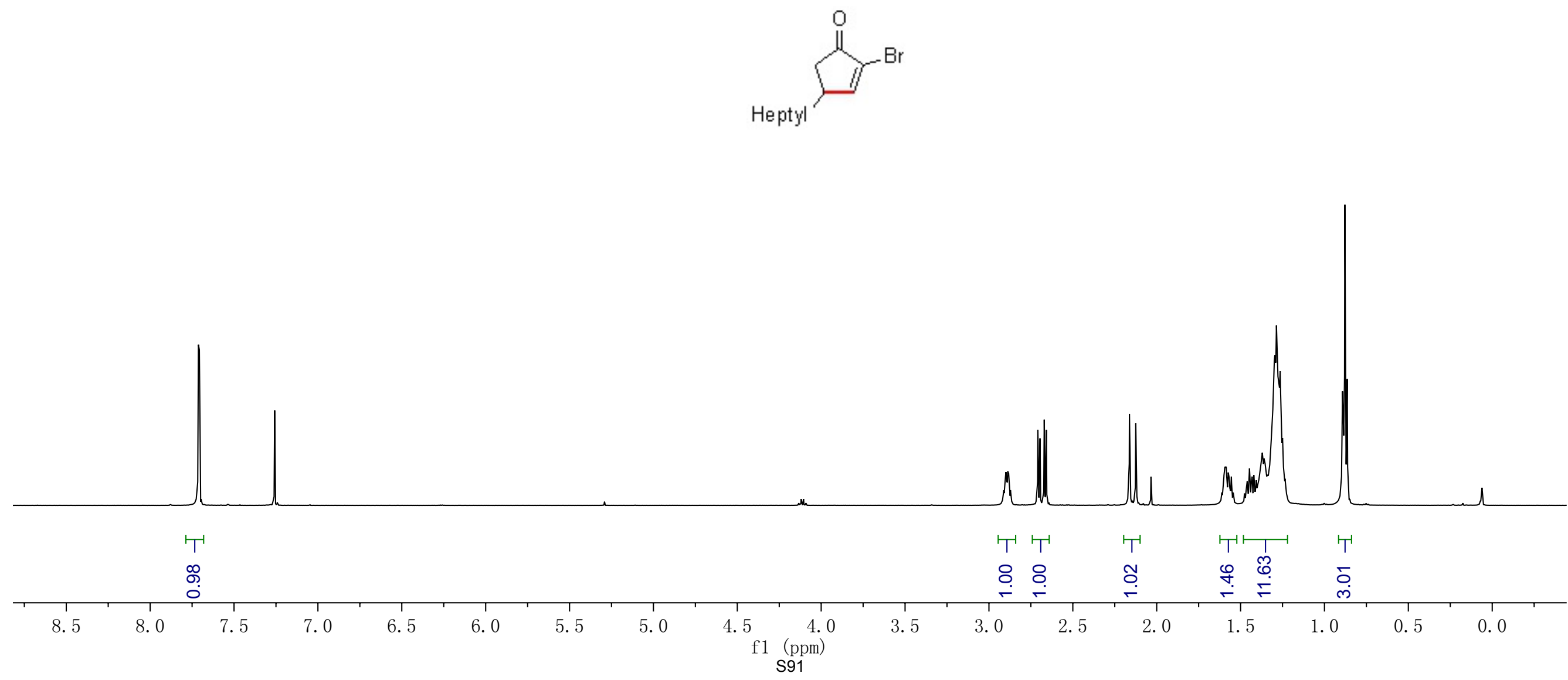
2 Solvent

- $298 \mathrm{~B}-\mathrm{P}$-again-C13d

Spectrometer Frequency 125.70

4 Nucleus $13 \mathrm{C}$<smiles>[14CH2]C1C=C(Br)C(=O)C1</smiles>

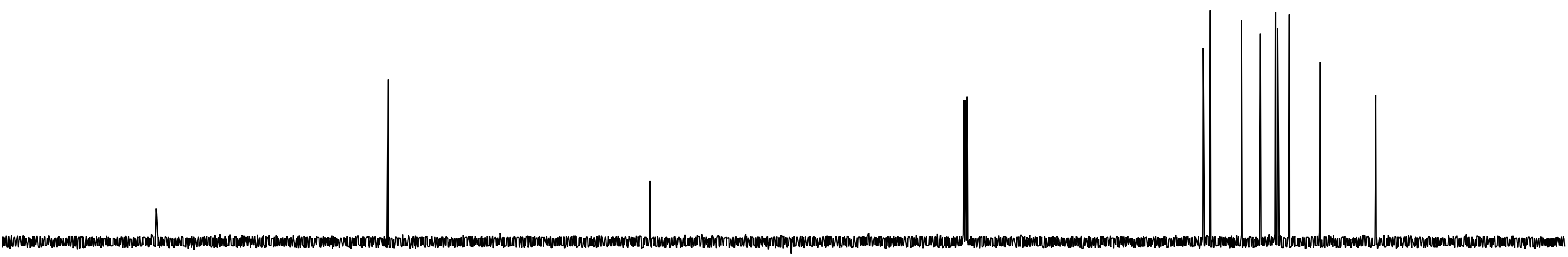

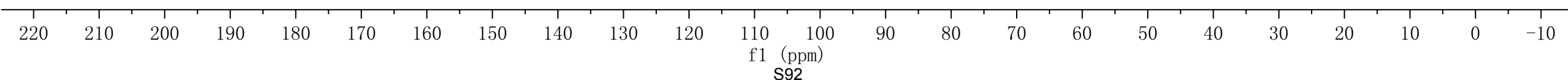




\begin{tabular}{|ll|}
\hline \multicolumn{1}{|c|}{ Parameter } & \multicolumn{1}{c|}{ Value } \\
1 Title & youliang-6-18B-P-500NMR \\
2 Solvent & $\mathrm{CDCl3}$ \\
3 Temperature & 25.0 \\
4 Relaxation Delay & 10.0000 \\
5 Spectrometer Frequency & 499.86 \\
\hline
\end{tabular}
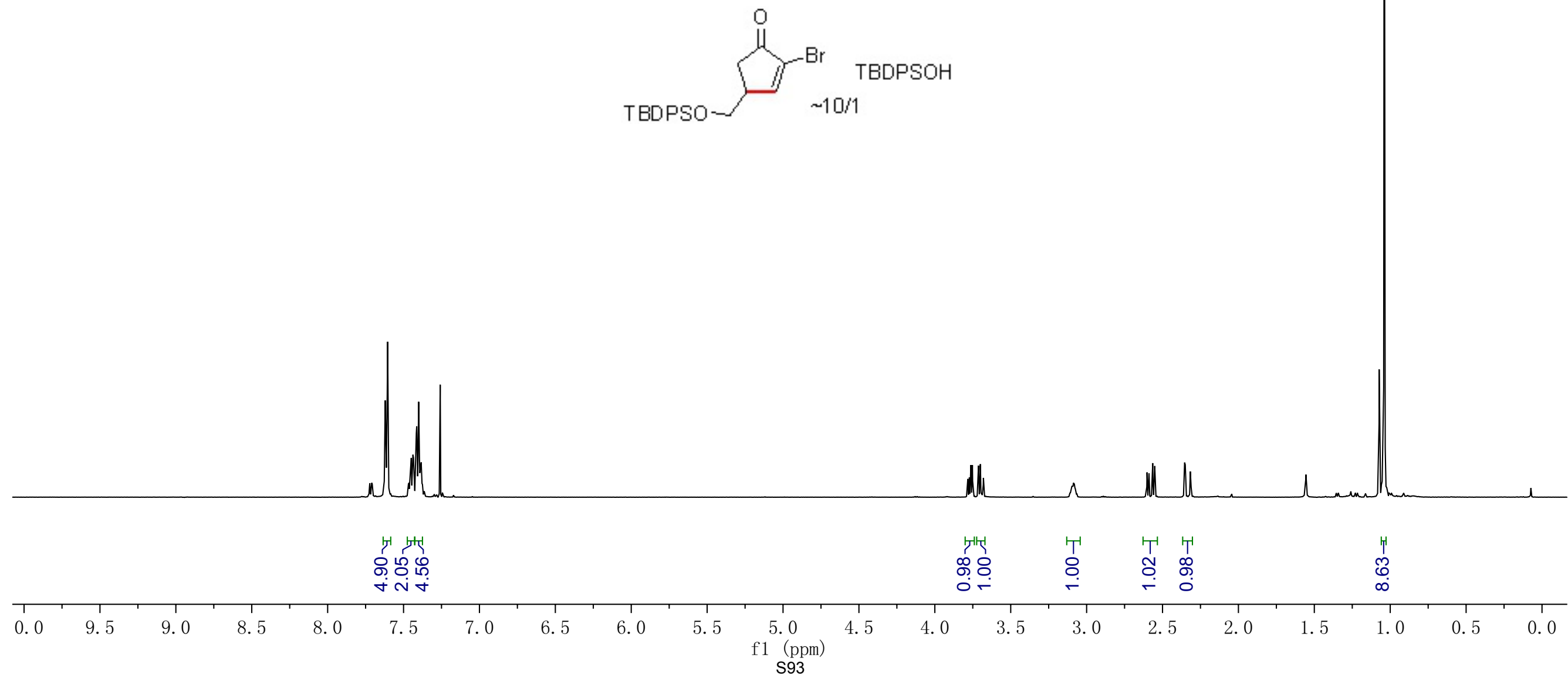


\begin{tabular}{|ll|}
\hline \multicolumn{1}{|c|}{ Parameter } & \multicolumn{1}{c|}{ Value } \\
1 Title & youliang-6-18B-P-500NMR-C13f \\
2 Solvent & $\mathrm{CDCl3}$ \\
3 Temperature & 25.0 \\
4 Relaxation Delay & 1.0000 \\
5 Spectrometer Frequency & 125.70 \\
\hline
\end{tabular}
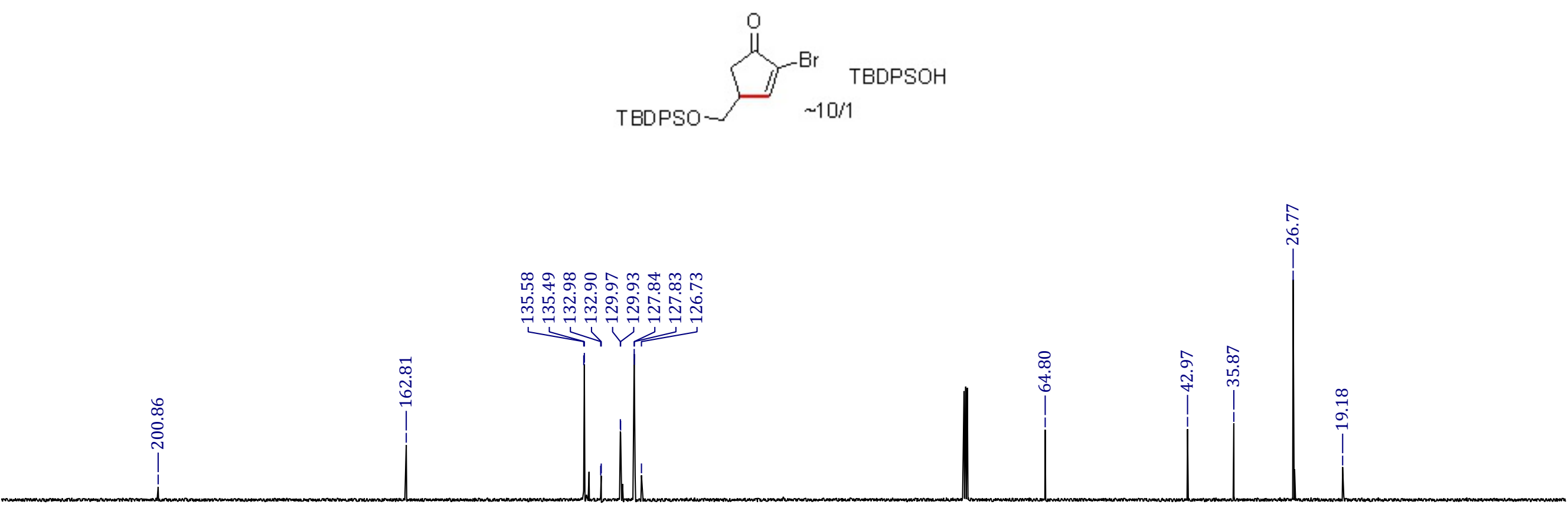

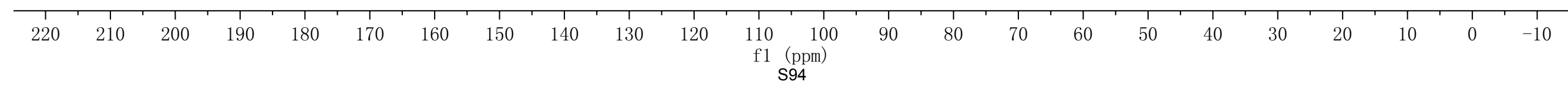




\begin{tabular}{|ll|}
\hline \multicolumn{1}{|c|}{ Parameter } & \multicolumn{1}{c|}{ Value } \\
1 Title & youliang-6-11B-P \\
2 Solvent & CDCl3 \\
3 Spectrometer Frequency & 499.86 \\
4 Nucleus & $1 \mathrm{H}$ \\
\hline
\end{tabular}
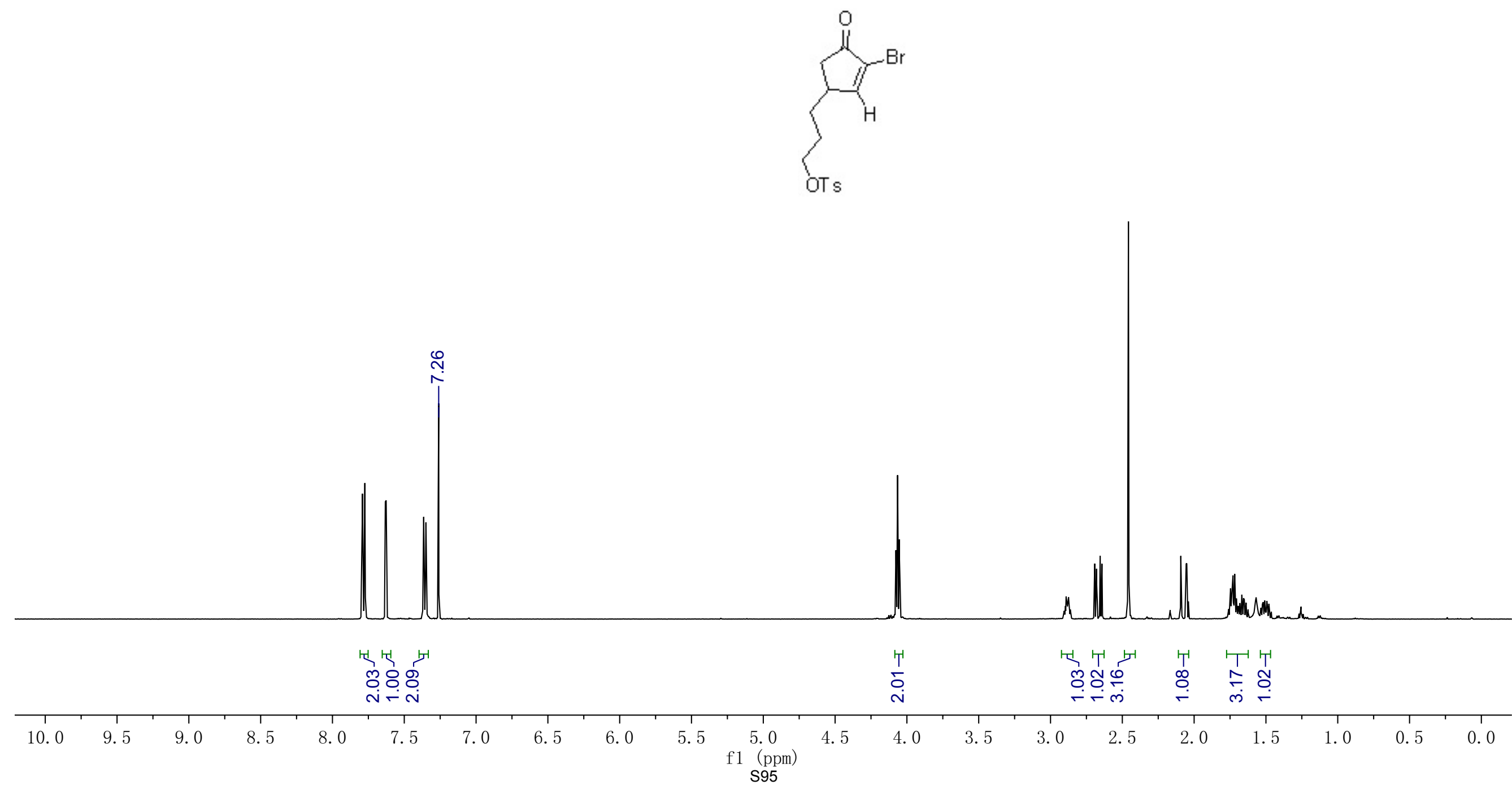


\begin{tabular}{|ll|}
\hline \multicolumn{1}{|c|}{ Parameter } & \multicolumn{1}{c|}{ Value } \\
1 Title & youliang-6-11B-P-C13f \\
2 Solvent & $\mathrm{CDCl} 3$ \\
3 Spectrometer Frequency & 125.70 \\
4 Nucleus & $13 \mathrm{C}$ \\
\hline
\end{tabular}
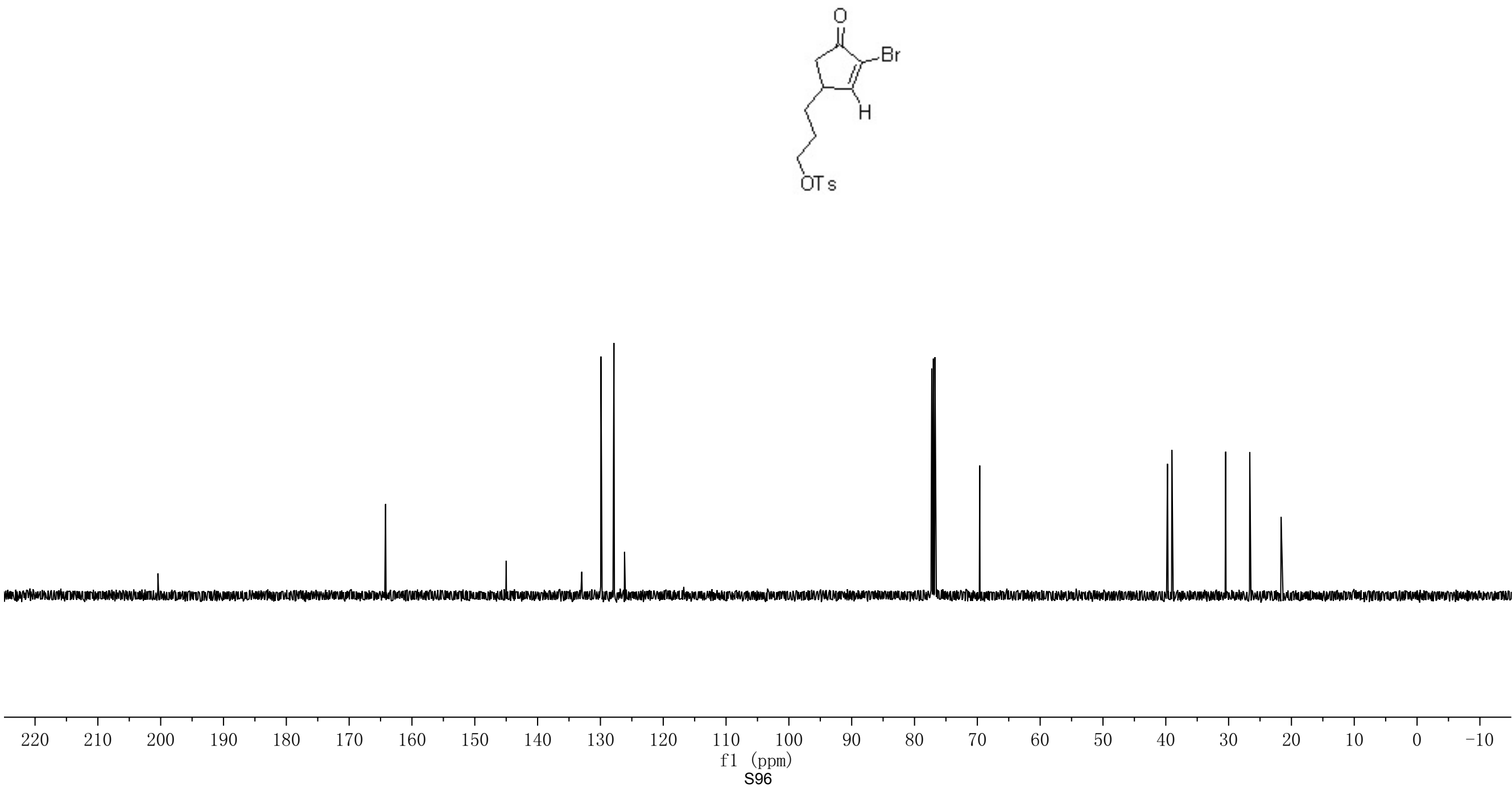


\begin{tabular}{|ll|}
\hline \multicolumn{1}{|c|}{ Parameter } & \multicolumn{1}{c|}{ Value } \\
1 Title & youliang-6-15C-P \\
2 Solvent & CDCl3 \\
3 Temperature & 25.0 \\
4 Relaxation Delay & 10.0000 \\
5 Spectrometer Frequency & 499.86 \\
\hline
\end{tabular}
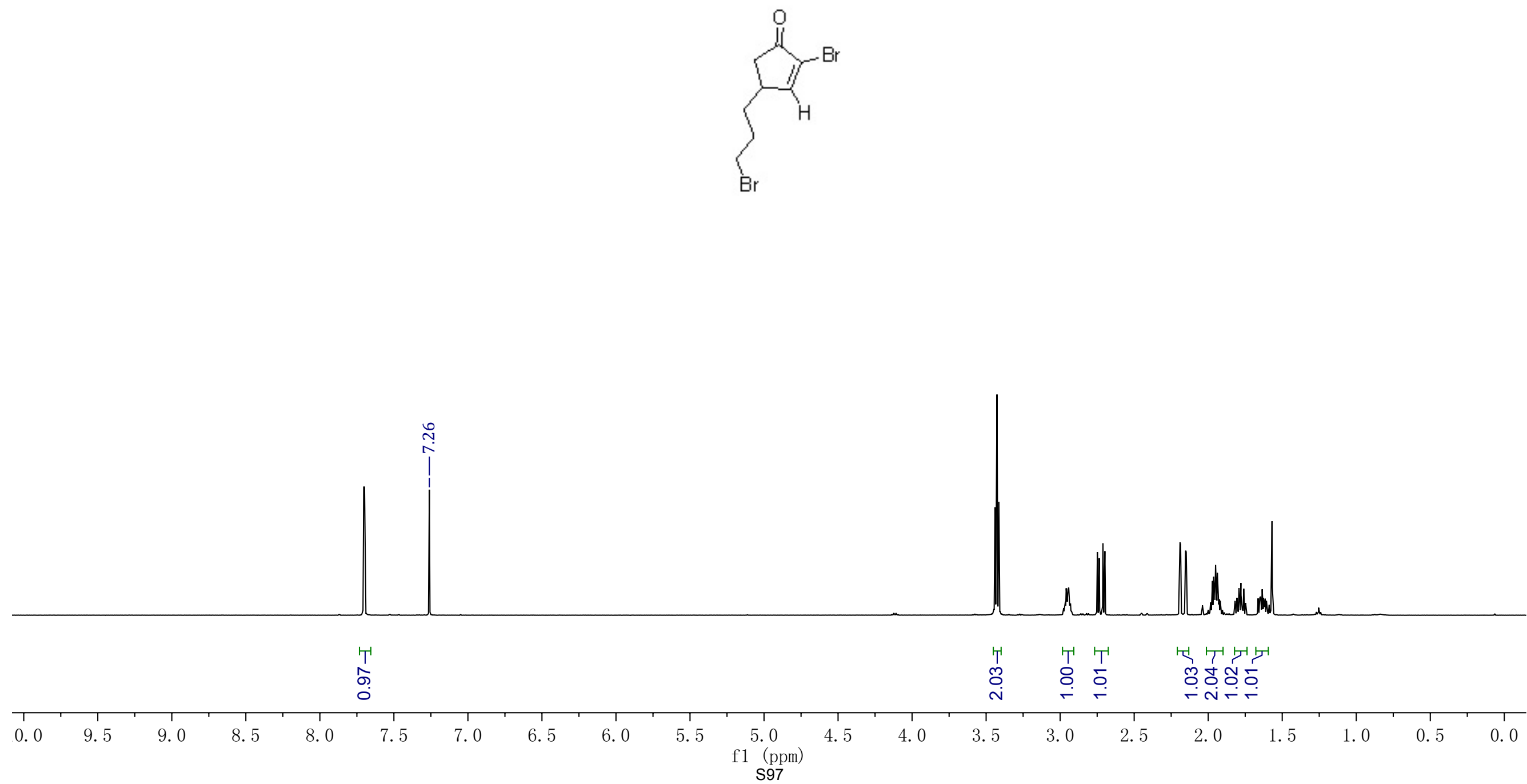


\begin{tabular}{|ll|}
\hline \multicolumn{1}{|c|}{ Parameter } & \multicolumn{1}{c|}{ Value } \\
1 Title & youliang-6-15C-P-C13f \\
2 Solvent & $\mathrm{CDCl} 3$ \\
3 Temperature & 25.0 \\
4 Relaxation Delay & 1.0000 \\
5 Spectrometer Frequency & 125.70 \\
\hline
\end{tabular}
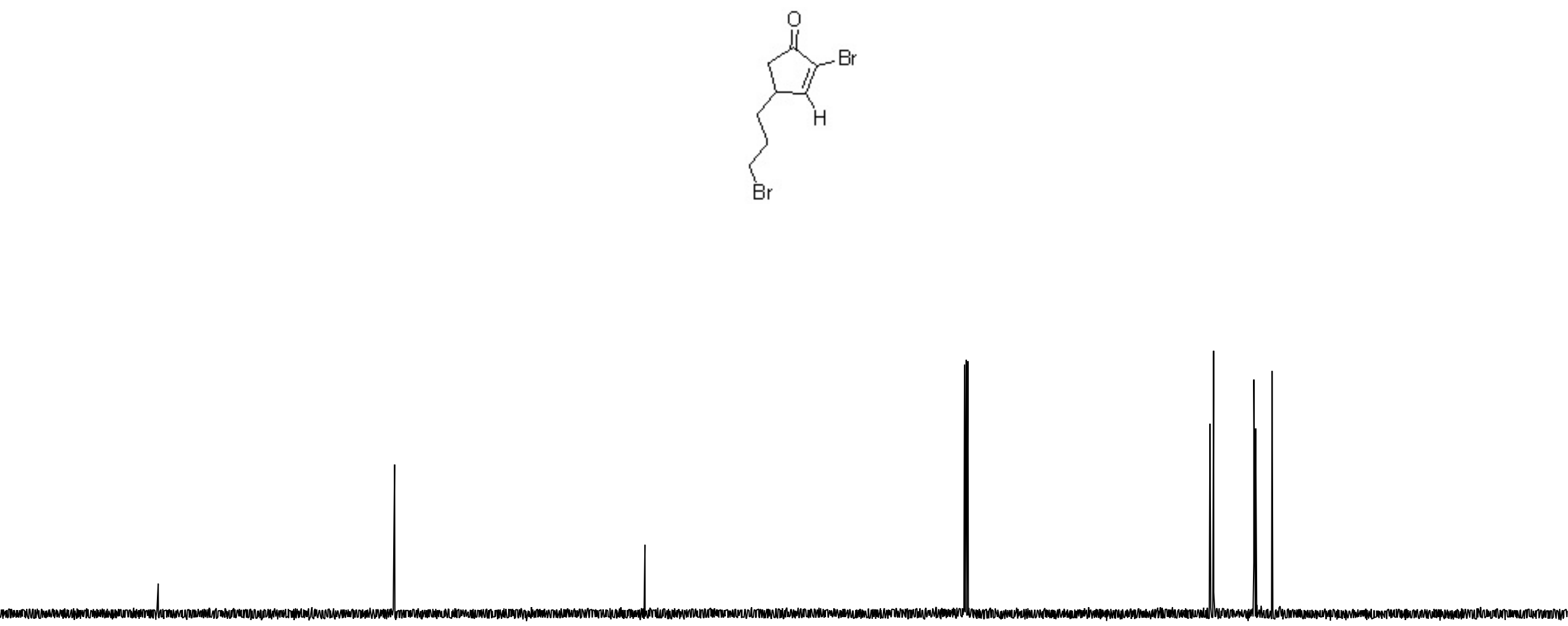

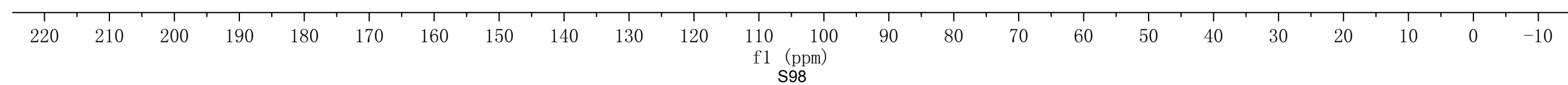




\begin{tabular}{|ll|}
\hline \multicolumn{1}{|c|}{ Parameter } & \multicolumn{1}{c|}{ Value } \\
1 Title & youliang-5-275-A-P \\
2 Solvent & $\mathrm{CDCl} 3$ \\
3 Temperature & 25.0 \\
4 Relaxation Delay & 10.0000 \\
5 Spectrometer Frequency & 499.86 \\
\hline
\end{tabular}
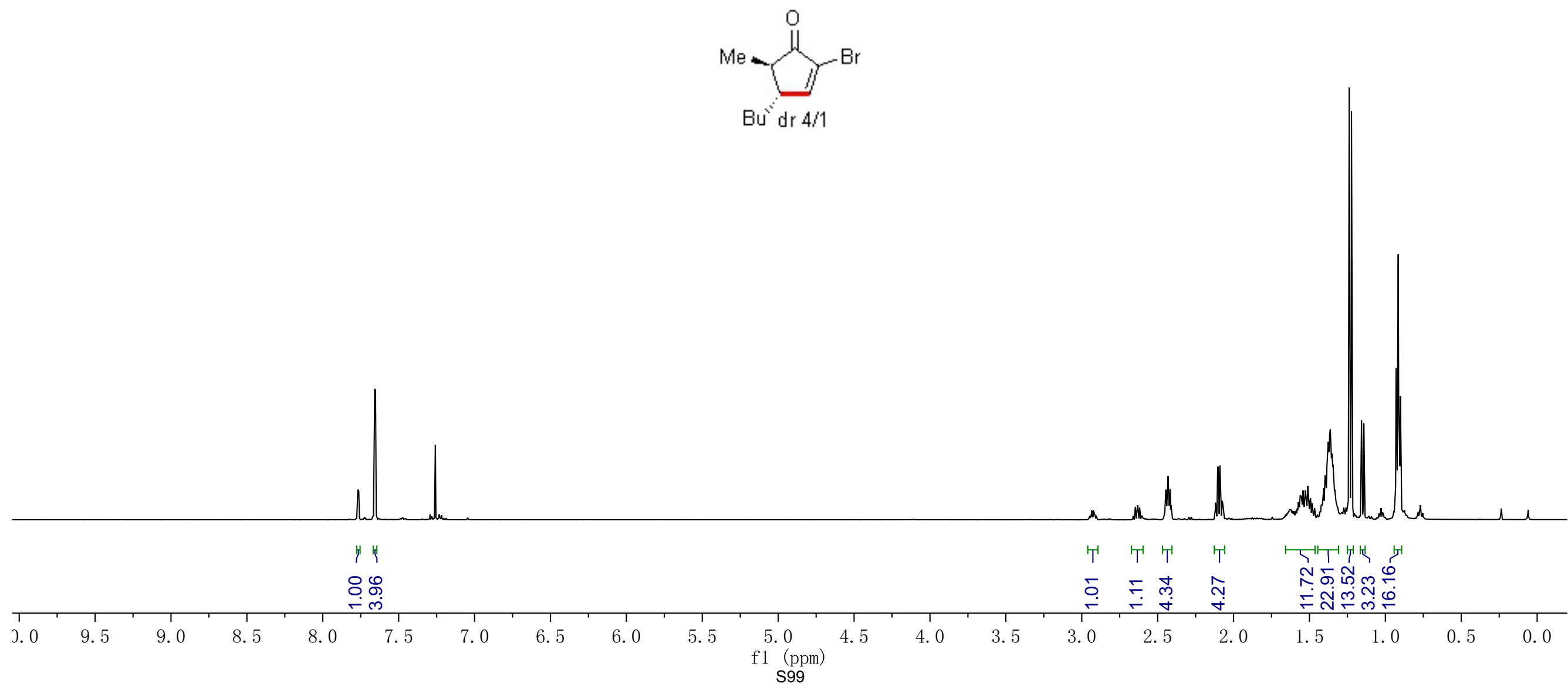


\begin{tabular}{|ll|}
\hline \multicolumn{1}{|c|}{ Parameter } & \multicolumn{1}{c|}{ Value } \\
1 Title & youliang-5-275-A-P-C13g \\
2 Solvent & CDCl3 \\
3 Temperature & 25.0 \\
4 Relaxation Delay & 1.0000 \\
5 Spectrometer Frequency & 125.70 \\
\hline
\end{tabular}
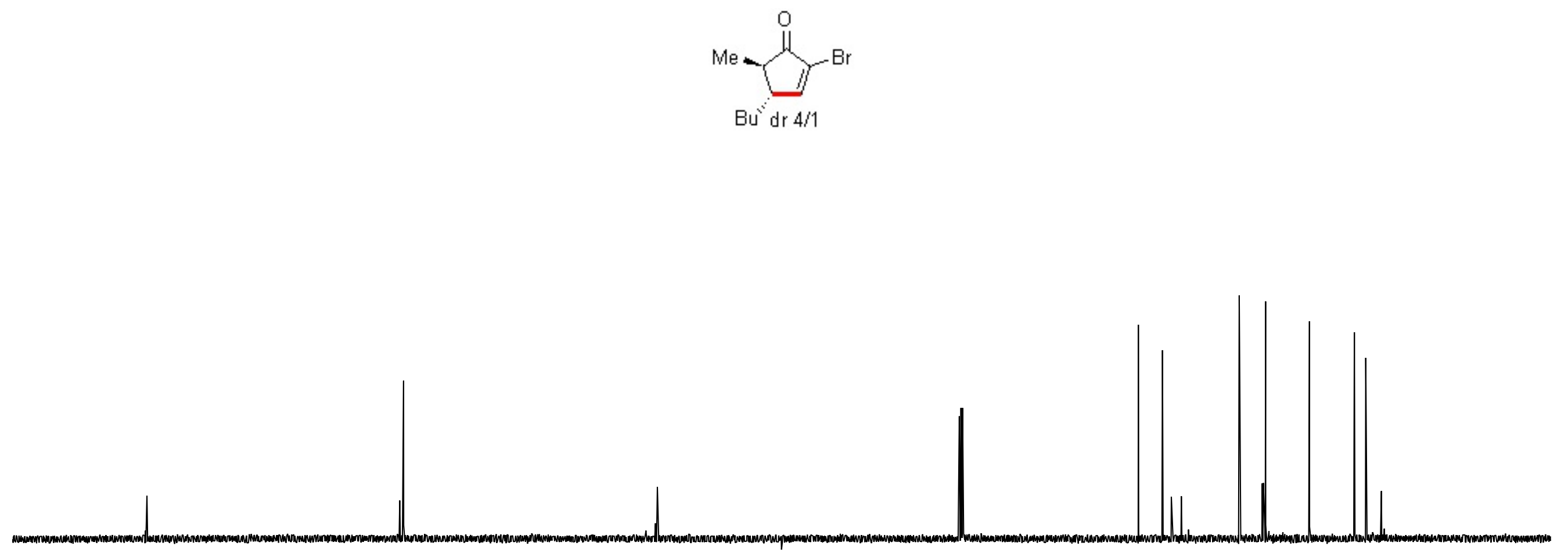

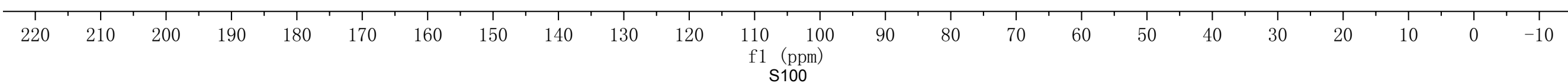




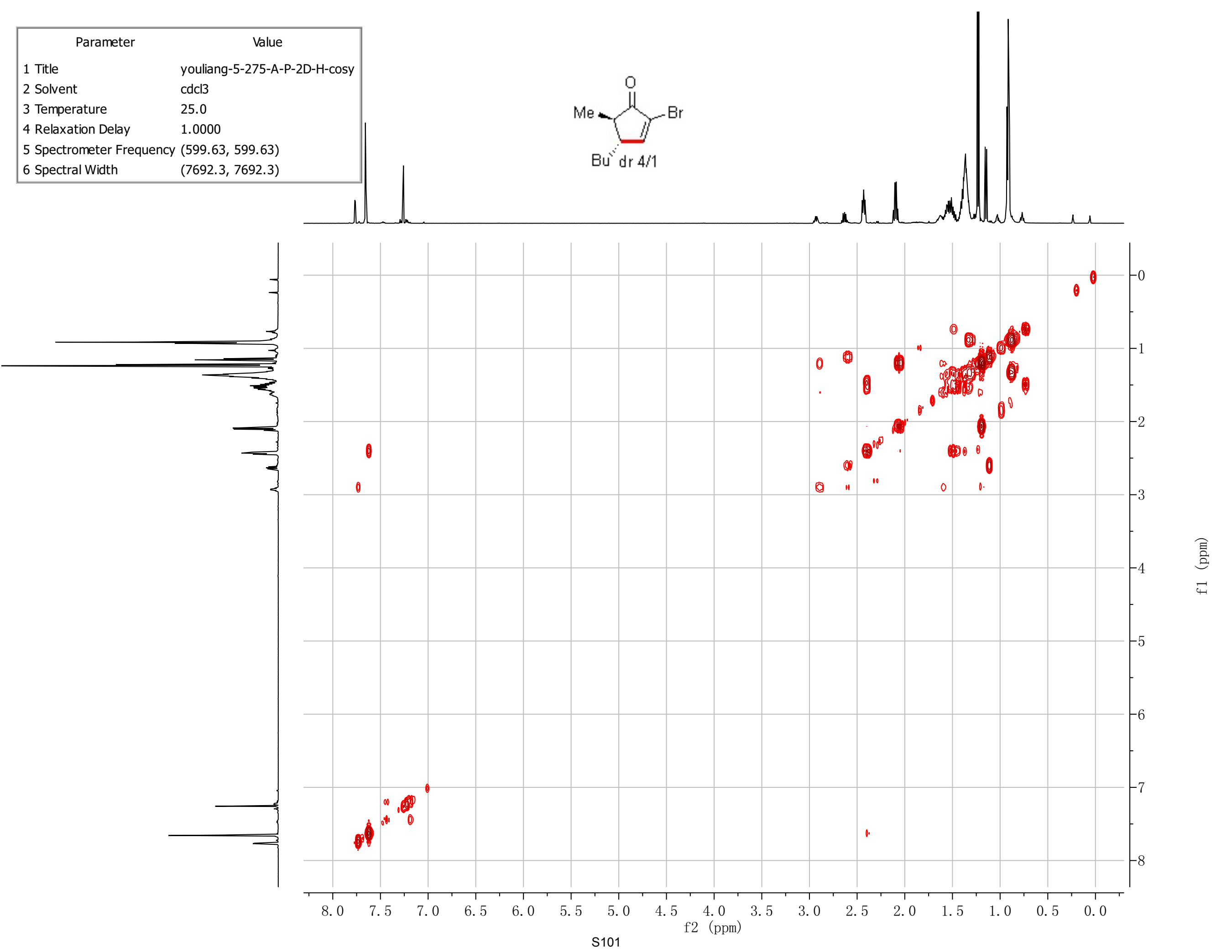




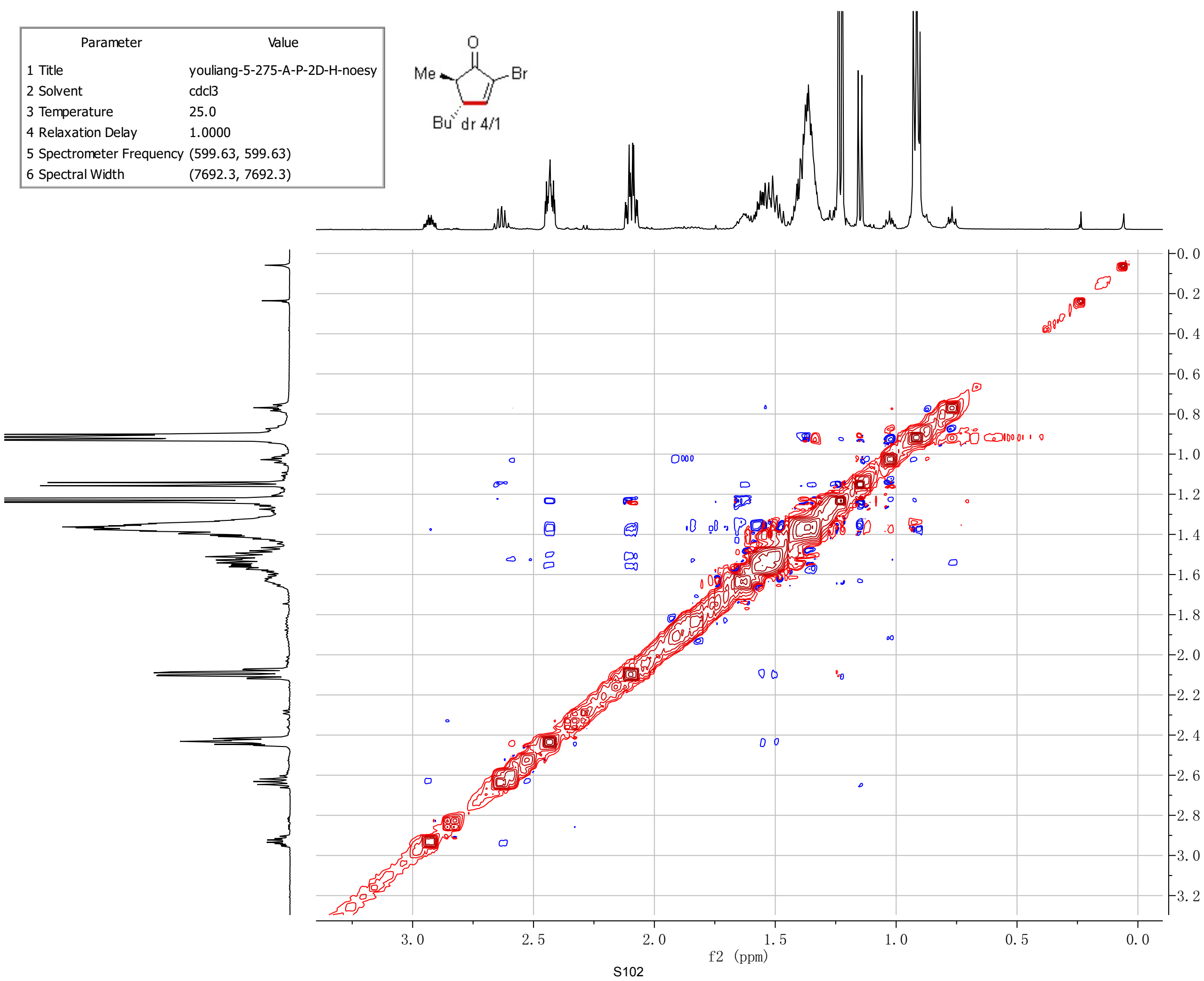




\begin{tabular}{|ll|}
\hline \multicolumn{1}{|c|}{ Parameter } & \multicolumn{1}{c|}{ Value } \\
1 Title & youliang-6-25B-P \\
2 Solvent & CDCl3 \\
3 Spectrometer Frequency & 499.86 \\
4 Nucleus & $1 \mathrm{H}$ \\
\hline
\end{tabular}
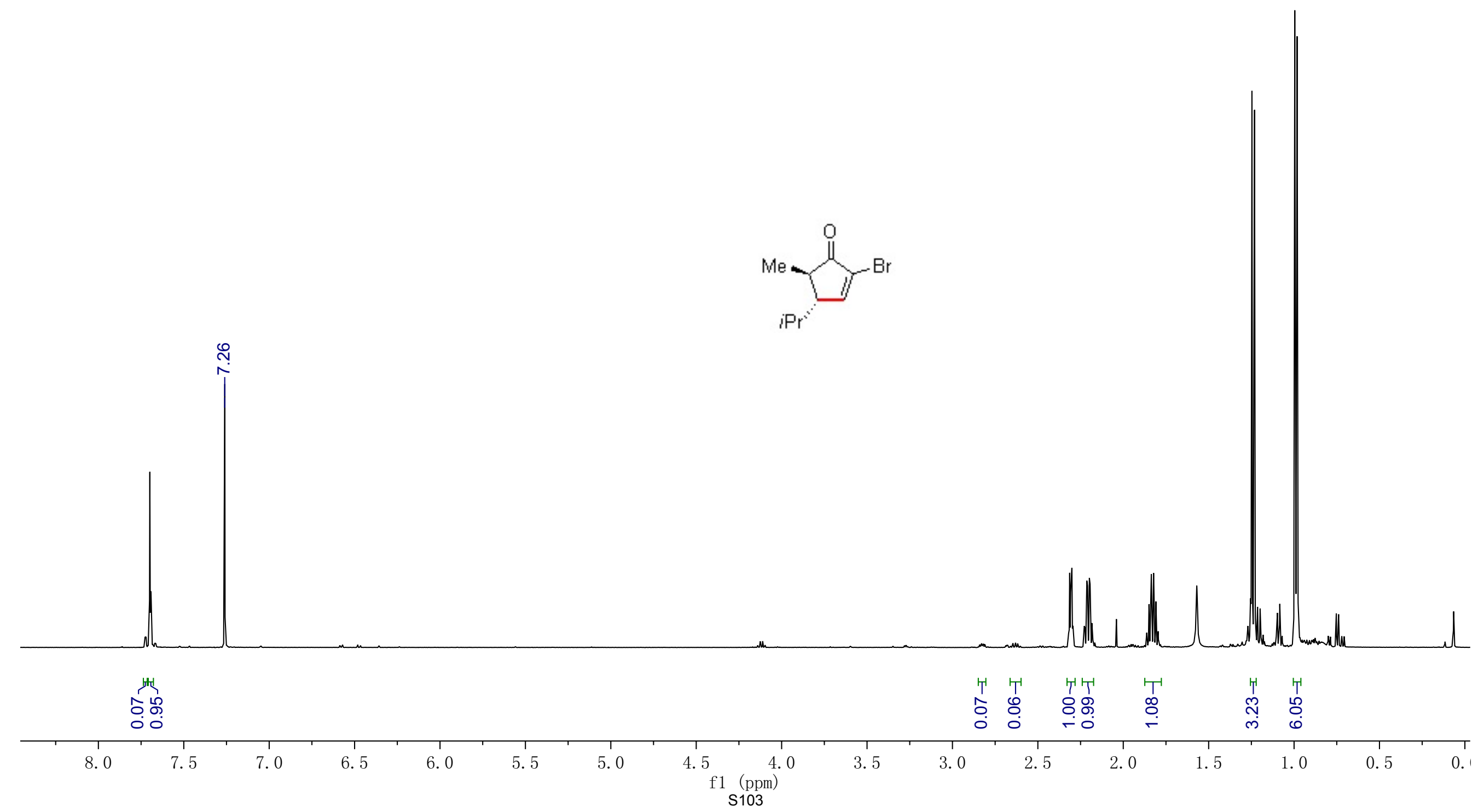


\begin{tabular}{|ll|}
\hline \multicolumn{1}{|c|}{ Parameter } & \multicolumn{1}{c|}{ Value } \\
1 Title & youliang-6-25B-P-C13i \\
2 Solvent & $\mathrm{CDCl3}$ \\
3 Spectrometer Frequency & 125.70 \\
4 Nucleus & $13 \mathrm{C}$ \\
\hline
\end{tabular}
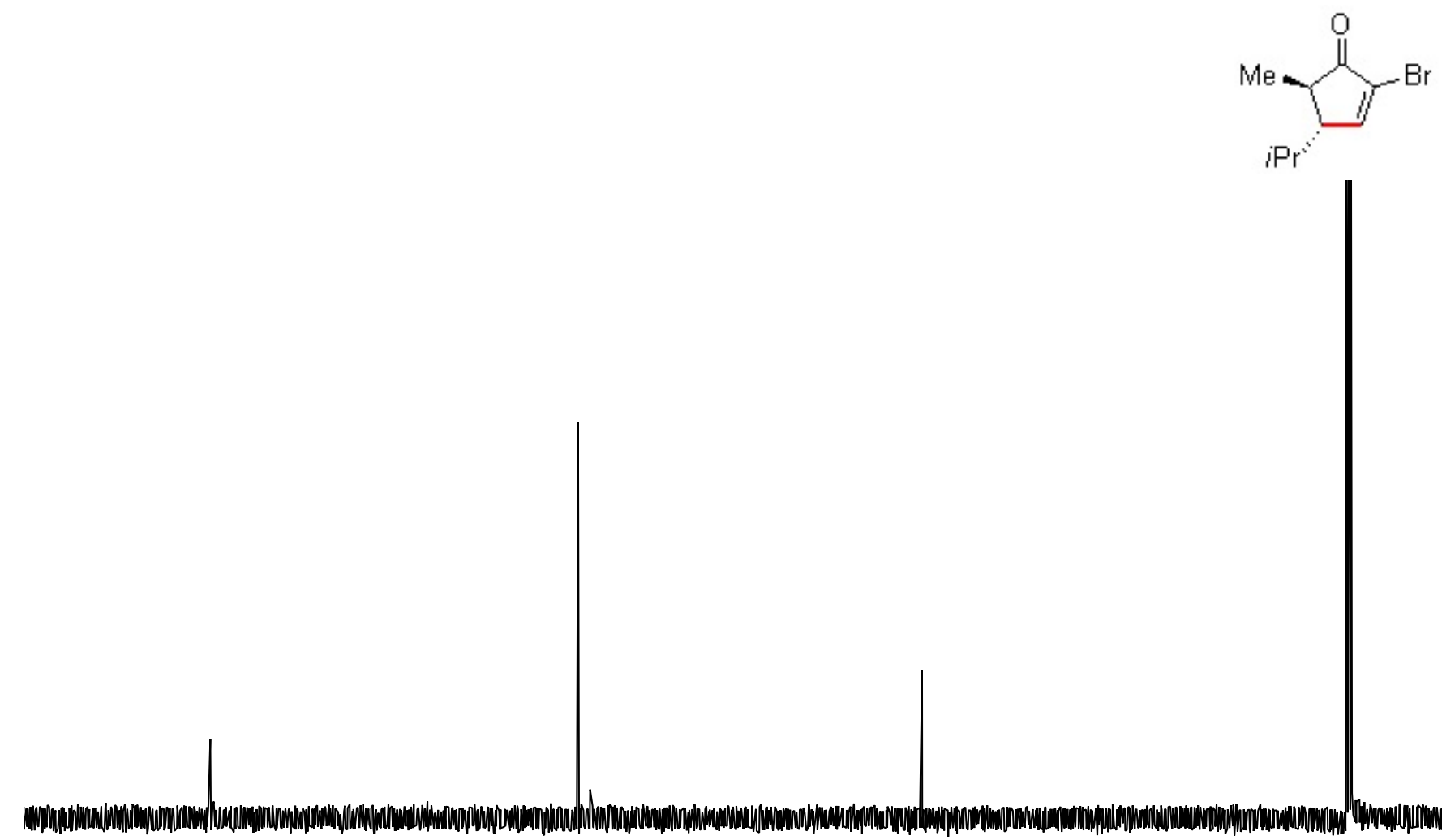

\begin{tabular}{|c|c|c|c|c|c|c|c|c|c|c|c|c|c|c|c|c|c|c|c|c|c|c|c|}
\hline 220 & 210 & 200 & 190 & 180 & 170 & 160 & 150 & 140 & 130 & 120 & $\begin{array}{r}110 \\
\mathrm{f} 1\end{array}$ & $\begin{array}{l}100 \\
(\mathrm{ppm}) \\
104\end{array}$ & 90 & 80 & 70 & 60 & 50 & 40 & 30 & 20 & 10 & 0 & -10 \\
\hline
\end{tabular}




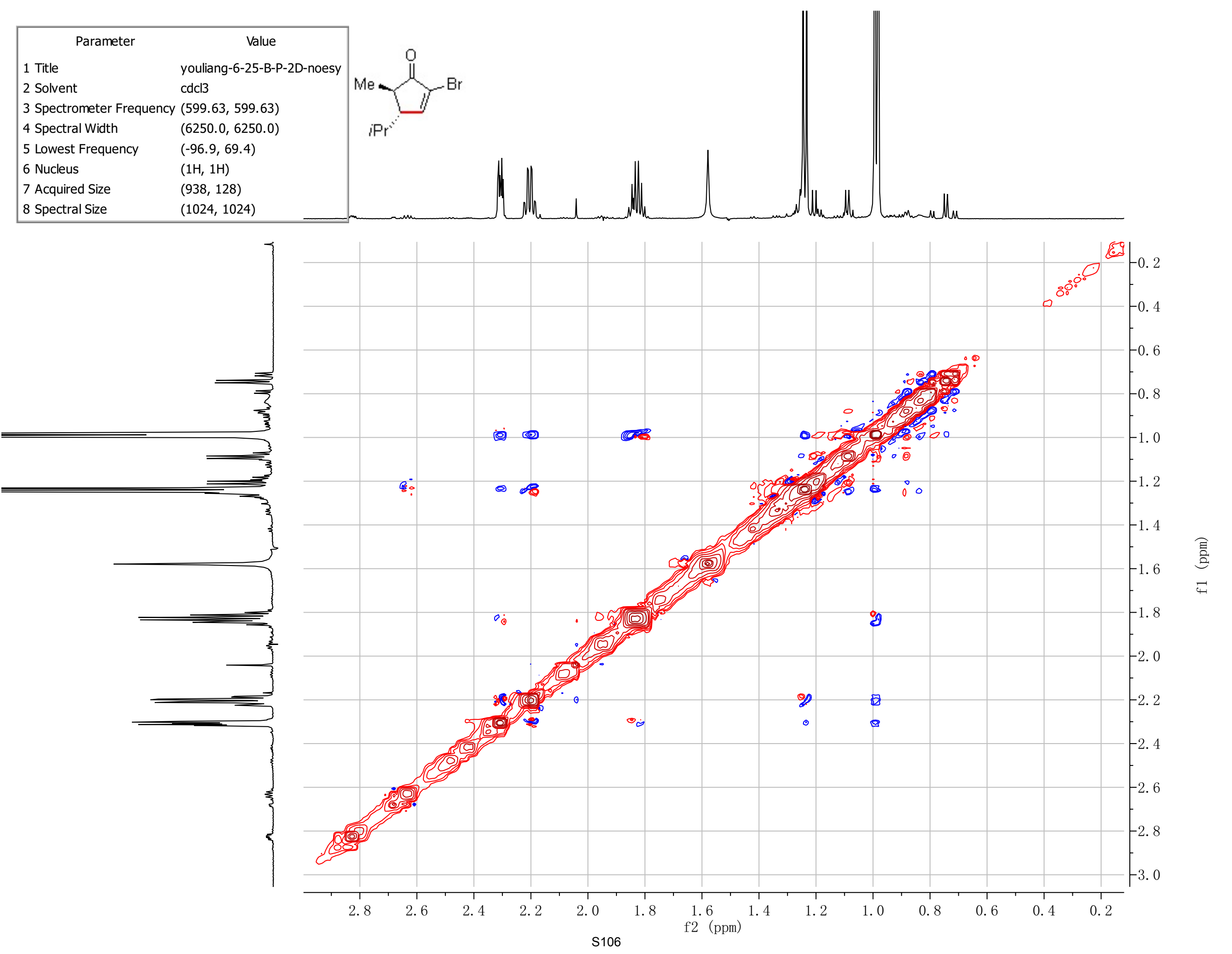




\begin{tabular}{|ll|}
\hline \multicolumn{1}{|c|}{ Parameter } & \multicolumn{1}{c|}{ Value } \\
1 Title & youliang-6-22C-P1 \\
2 Solvent & cdcl3 \\
3 Temperature & 25.0 \\
4 Relaxation Delay & 4.8000 \\
5 Spectrometer Frequency & 599.63 \\
\hline
\end{tabular}
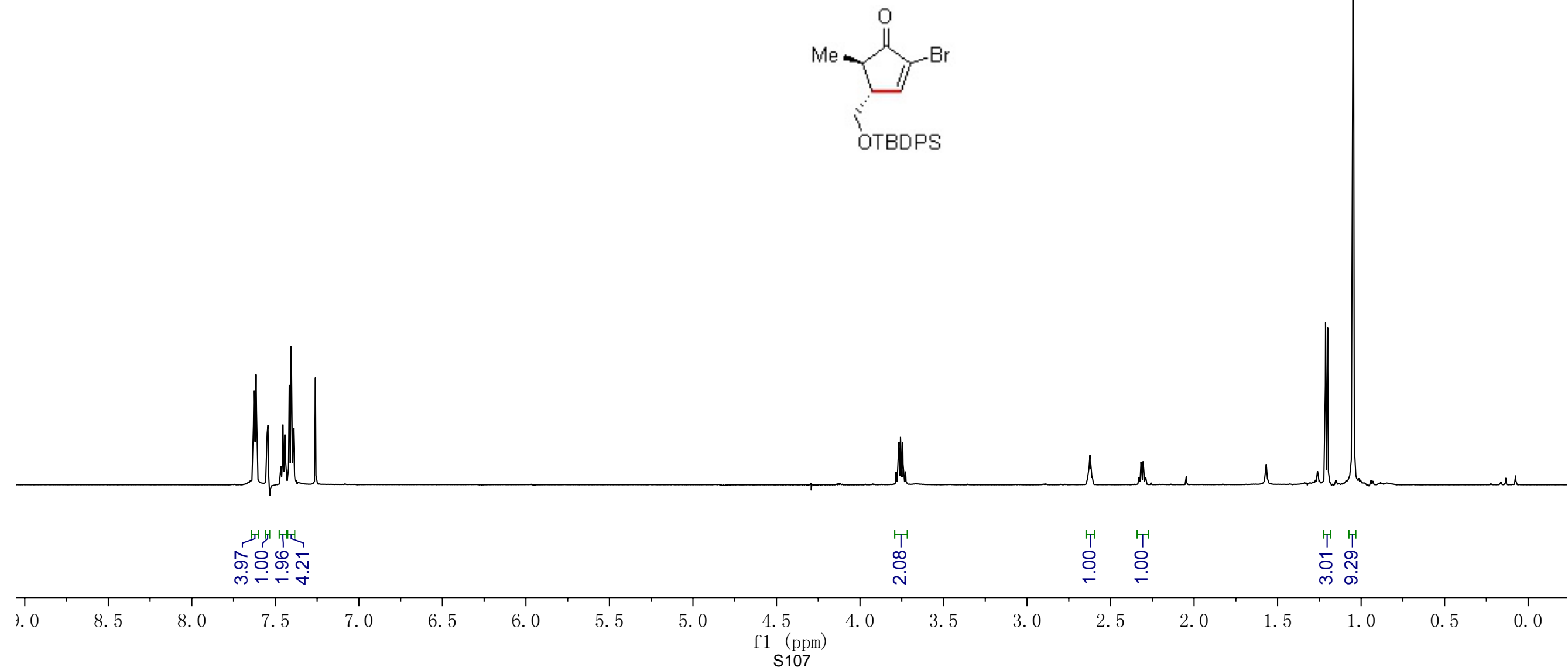


\begin{tabular}{|ll|}
\hline \multicolumn{1}{|c|}{ Parameter } & \multicolumn{1}{c|}{ Value } \\
1 Title & youliang-6-22B-P1-C13e \\
2 Solvent & CDCl3 \\
3 Temperature & 25.0 \\
4 Relaxation Delay & 1.0000 \\
5 Spectrometer Frequency & 125.70 \\
\hline
\end{tabular}
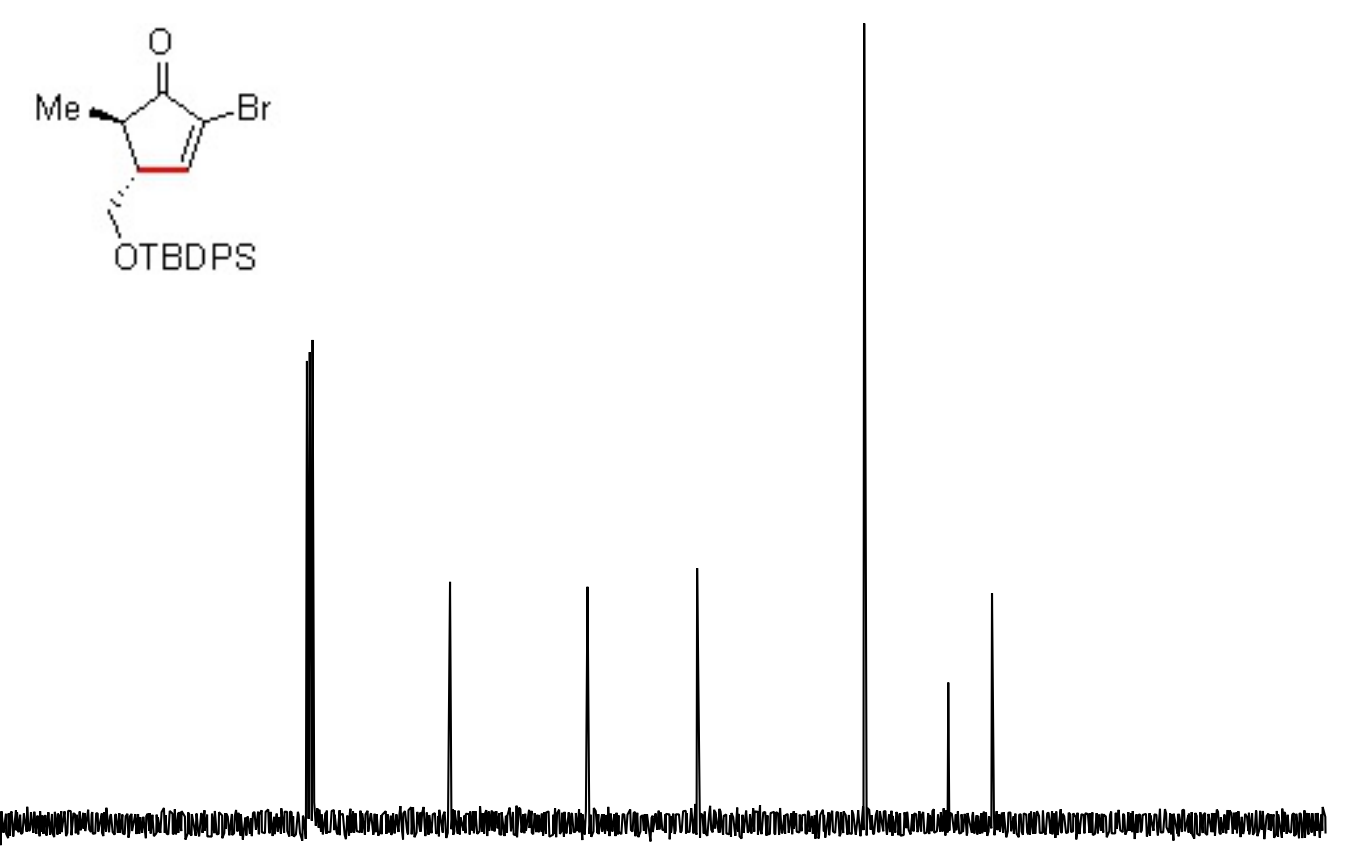

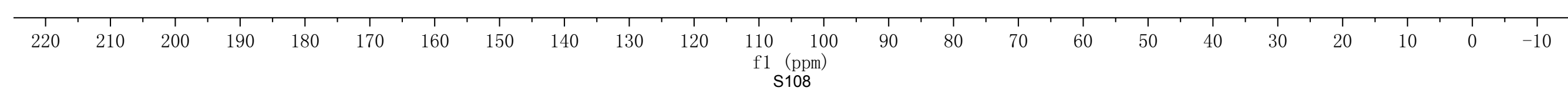




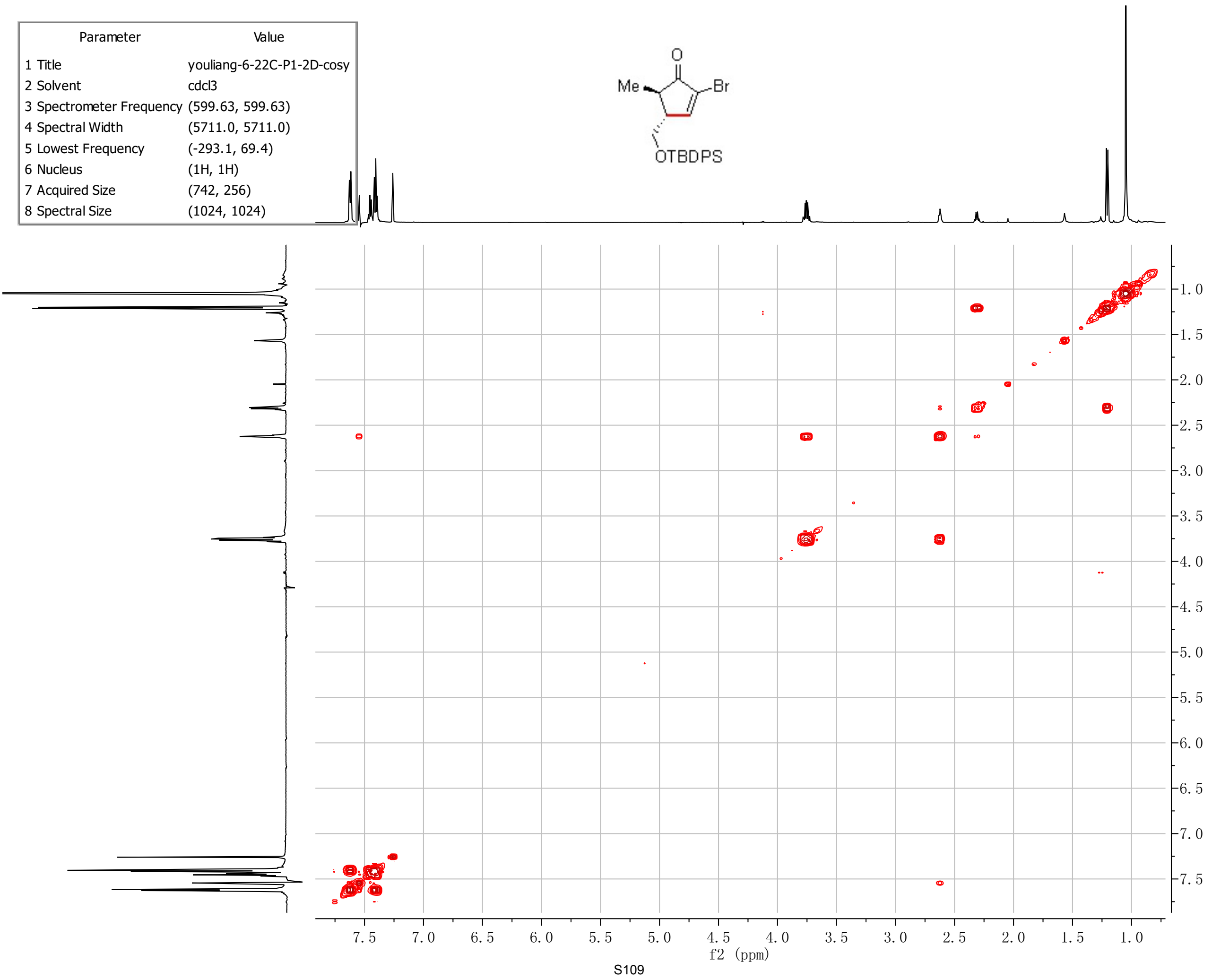




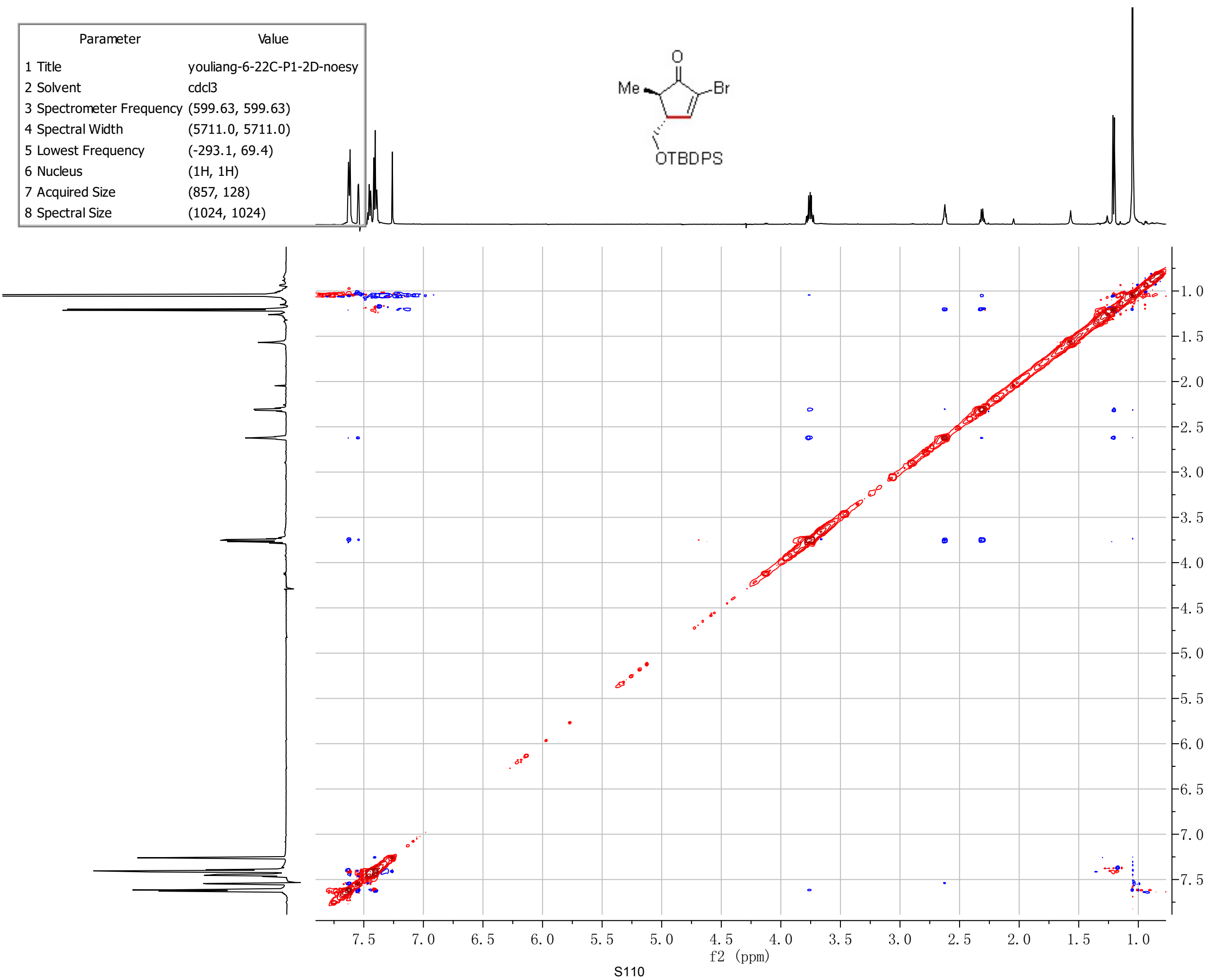




\begin{tabular}{|ll|}
\hline \multicolumn{1}{|c|}{ Parameter } & \multicolumn{1}{c|}{ Value } \\
1 Title & youliang-6-22C-P2-2nd \\
2 Solvent & $\mathrm{CDCl} 3$ \\
3 Spectrometer Frequency & 499.86 \\
4 Nucleus & $1 \mathrm{H}$ \\
\hline
\end{tabular}

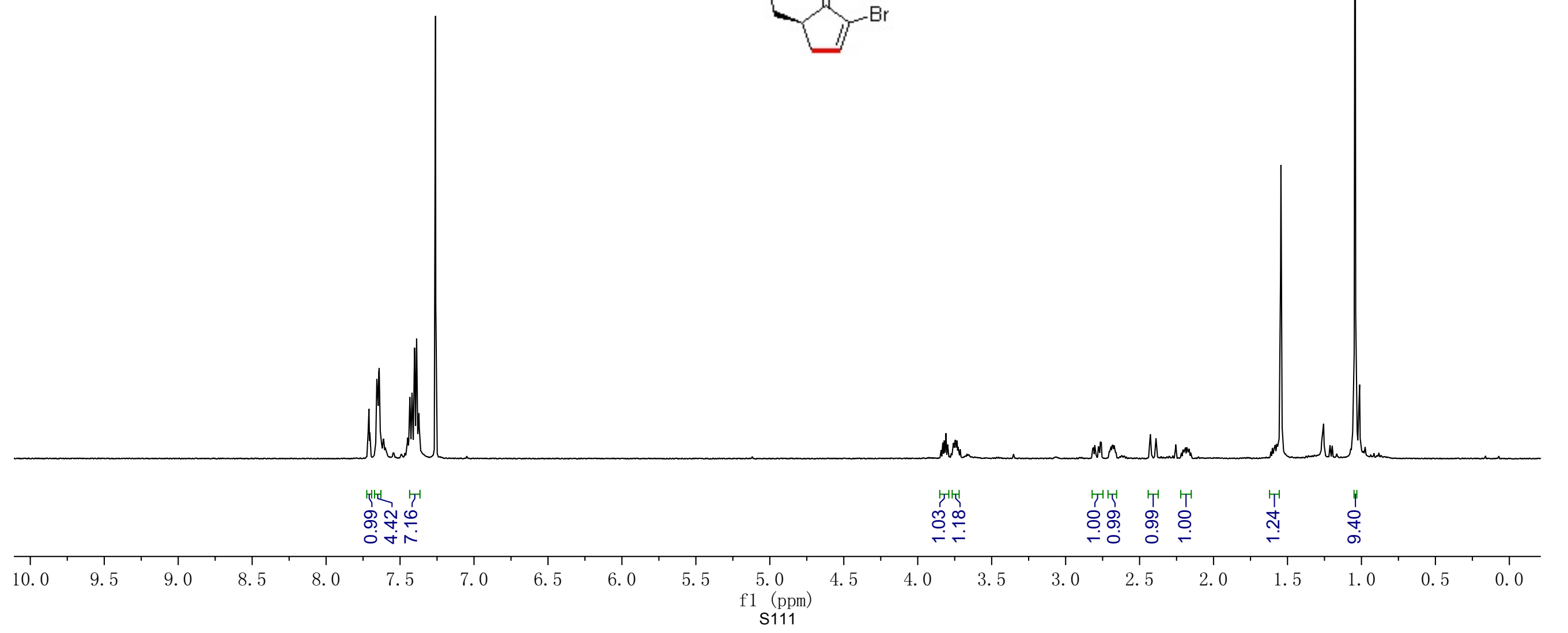




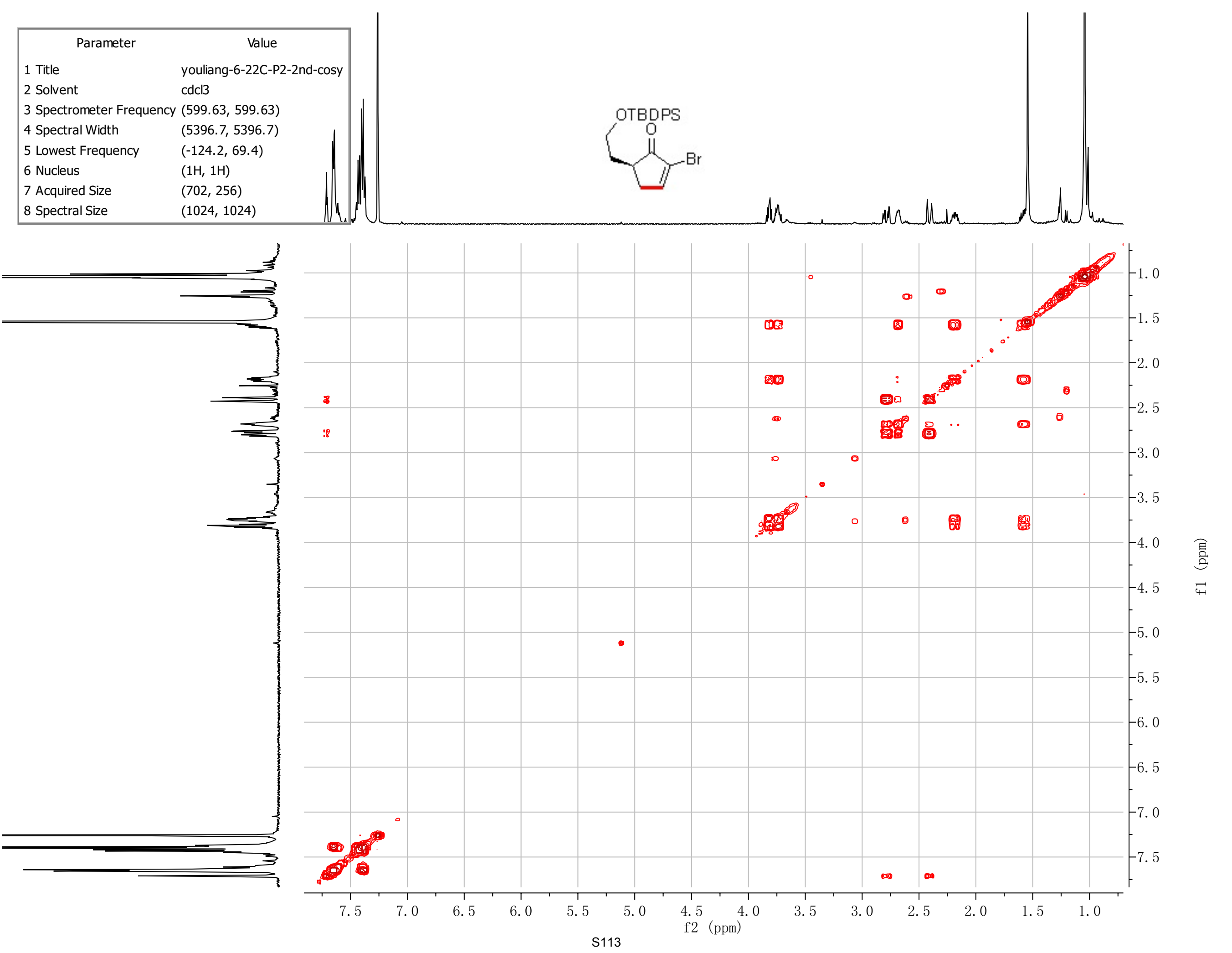




\begin{tabular}{|ll|}
\hline \multicolumn{1}{|c|}{ Parameter } & \multicolumn{1}{c|}{ Value } \\
1 Title & youliang-6-76C-P1 \\
2 Solvent & $\mathrm{CDCl3}$ \\
3 Spectrometer Frequency & 499.86 \\
4 Nucleus & $1 \mathrm{H}$ \\
\hline
\end{tabular}

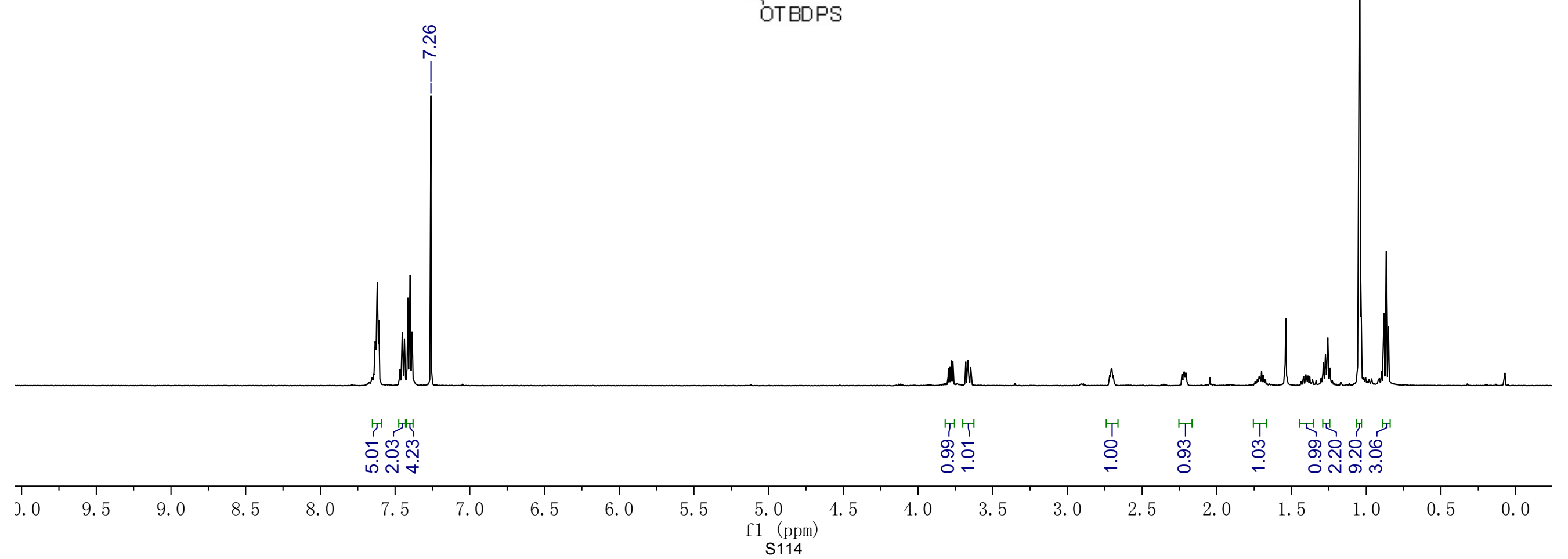




\begin{tabular}{|ll|}
\hline \multicolumn{1}{|c|}{ Parameter } & \multicolumn{1}{c|}{ Value } \\
1 Title & youliang-6-76C-P1-C13n \\
2 Solvent & $\mathrm{CDCl3}$ \\
3 Spectrometer Frequency & 125.70 \\
4 Nucleus & $13 \mathrm{C}$ \\
\hline
\end{tabular}

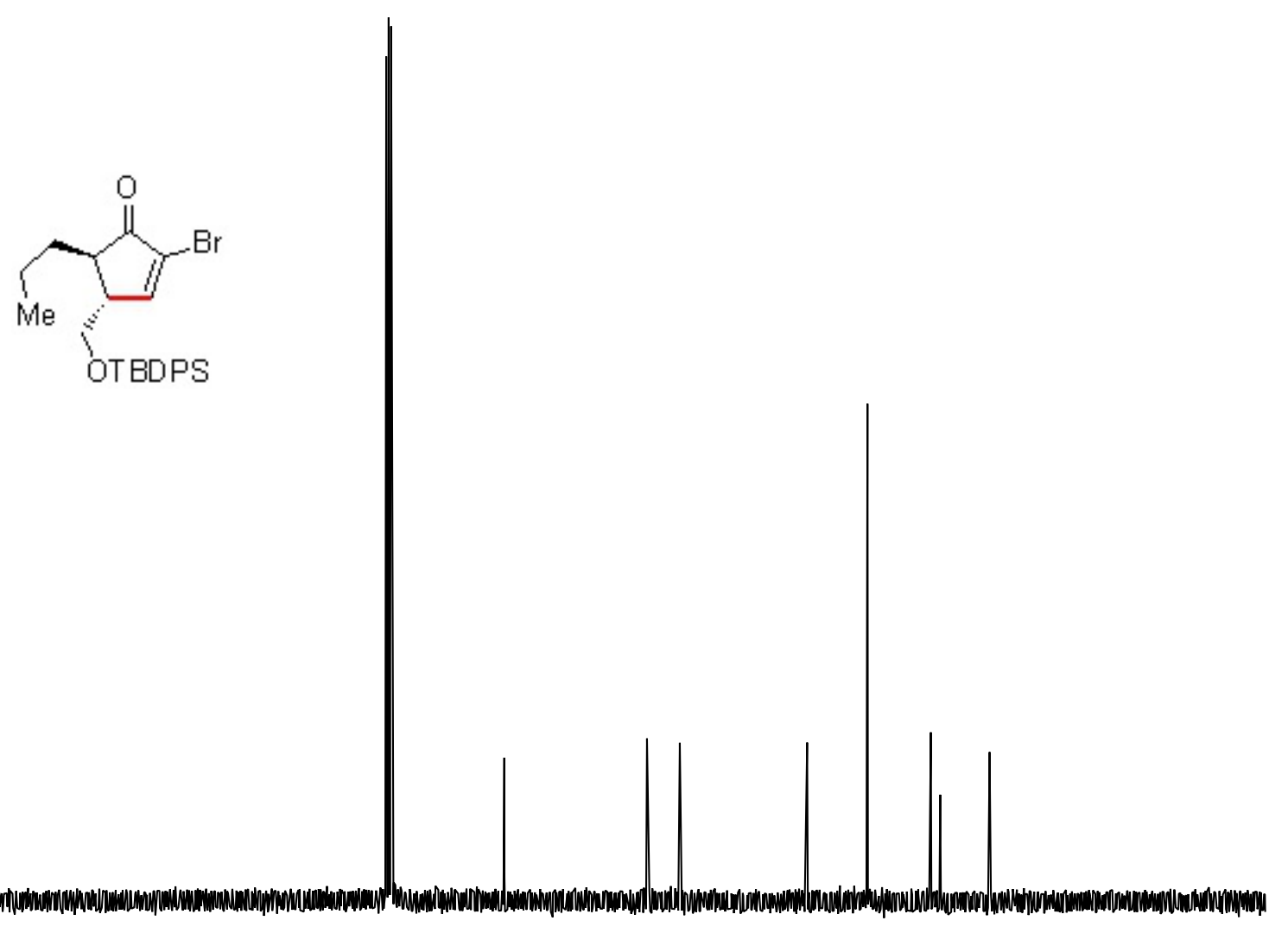

\begin{tabular}{|c|c|c|c|c|c|c|c|c|c|c|c|c|c|c|c|c|c|c|c|c|c|c|c|}
\hline $\begin{array}{l}1 \\
220\end{array}$ & 210 & 200 & 19 & $\begin{array}{l}1 \\
180\end{array}$ & $\begin{array}{l}1 \\
170\end{array}$ & 160 & 150 & 140 & $\begin{array}{l}1 \\
130\end{array}$ & 120 & $\begin{array}{c}110 \\
\text { f1 }\end{array}$ & $\begin{array}{l}1 \\
100 \\
\text { pm) } \\
15\end{array}$ & 90 & $\begin{array}{l}1 \\
80\end{array}$ & $\begin{array}{l}1 \\
70\end{array}$ & 190 & $\begin{array}{l}1 \\
50\end{array}$ & $\begin{array}{l}1 \\
40\end{array}$ & 30 & 20 & 10 & 0 & -10 \\
\hline
\end{tabular}




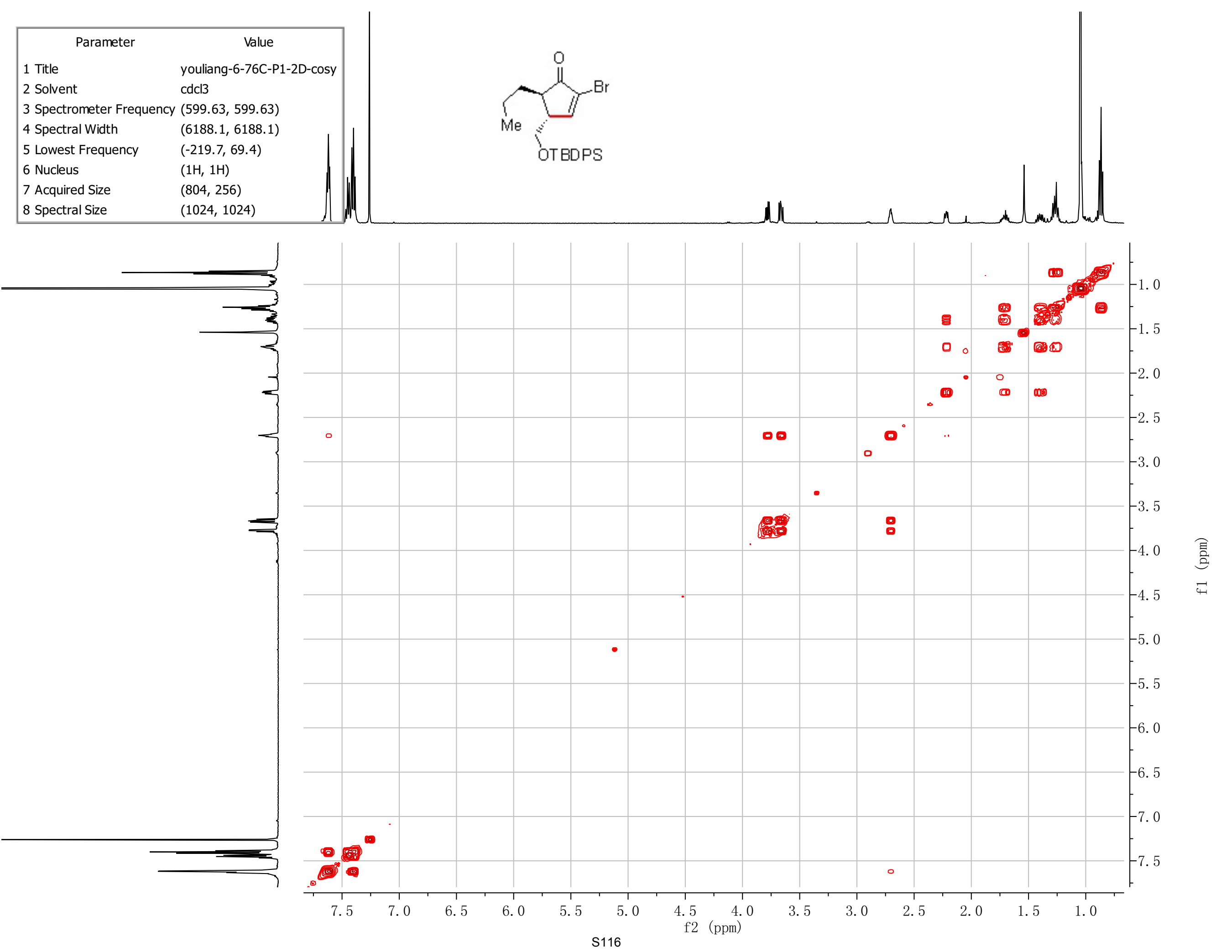




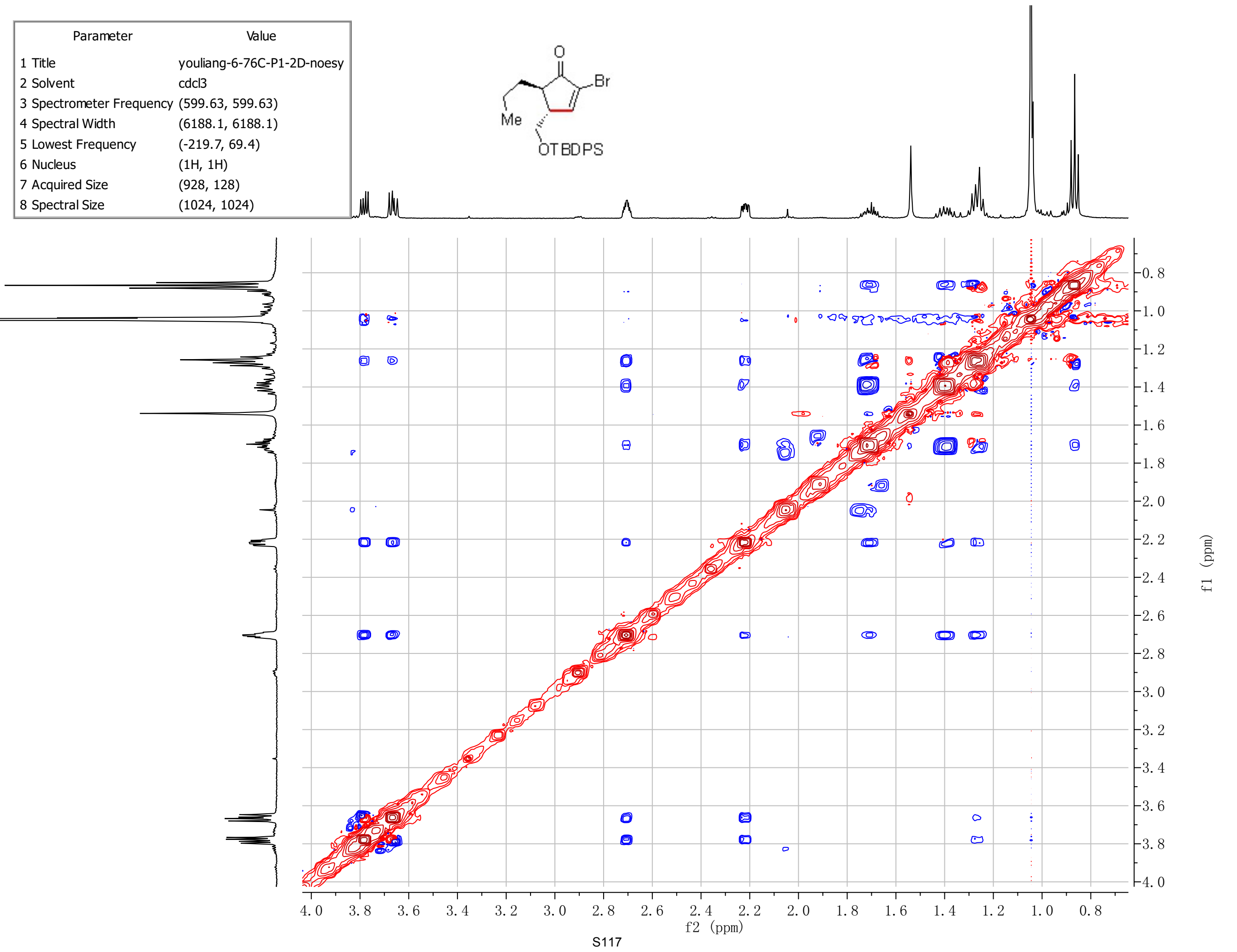




\begin{tabular}{|ll|}
\hline \multicolumn{1}{|c|}{ Parameter } & \multicolumn{1}{c|}{ Value } \\
1 Title & youliang-6-76C-P2 \\
2 Solvent & $\mathrm{CDCl3}$ \\
3 Spectrometer Frequency & 499.86 \\
4 Nucleus & $1 \mathrm{H}$ \\
\hline
\end{tabular}
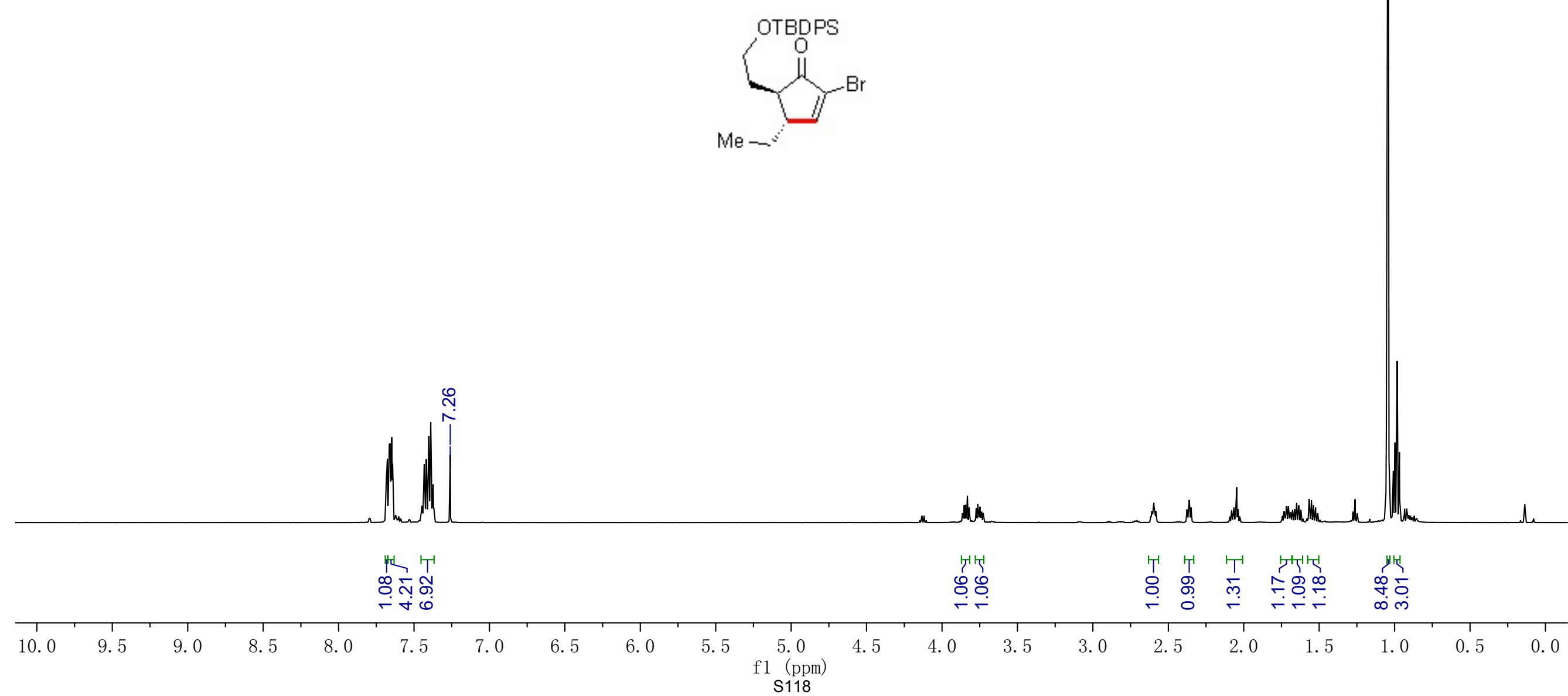
3 Spectrometer Frequency 125.70
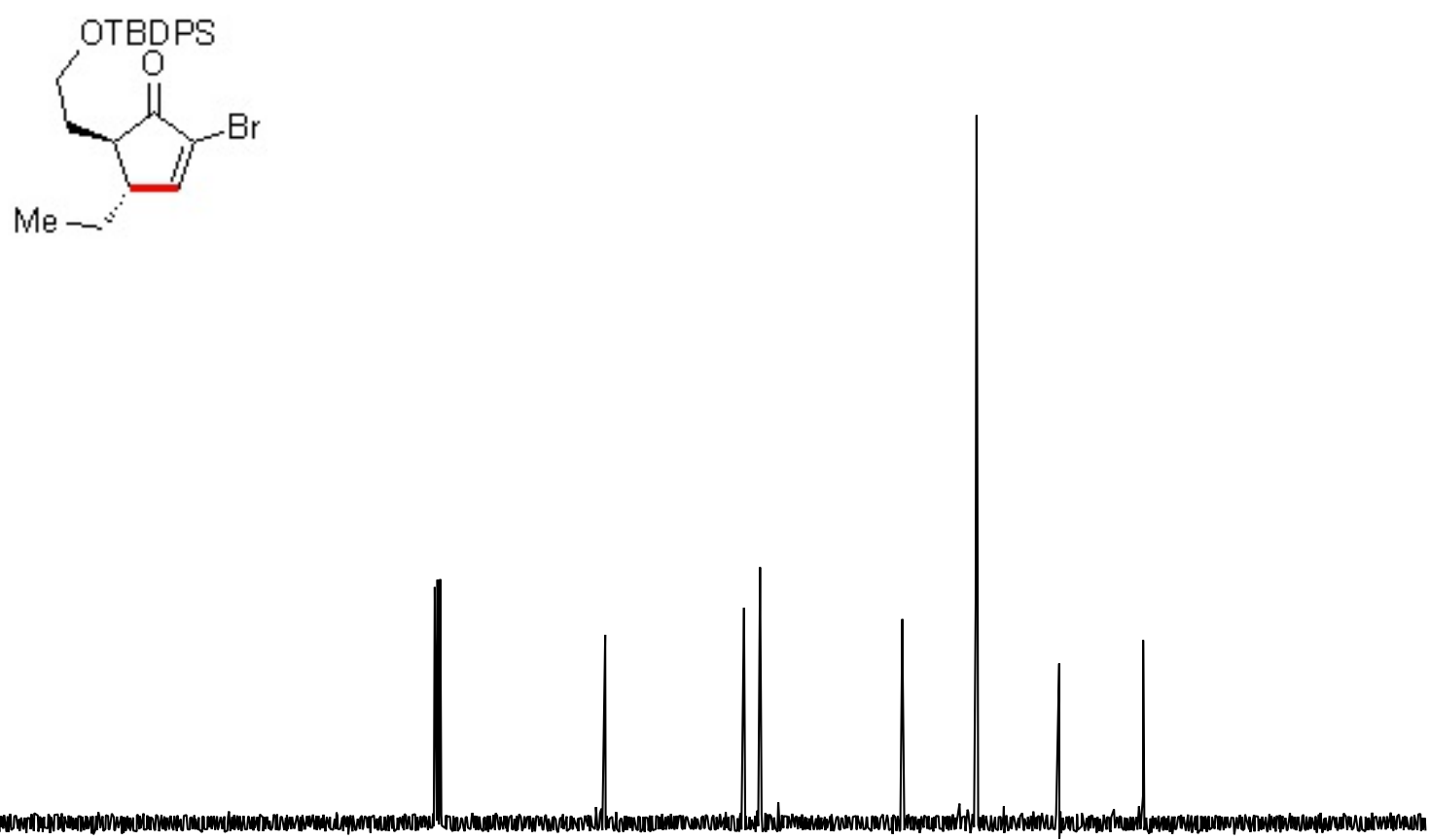

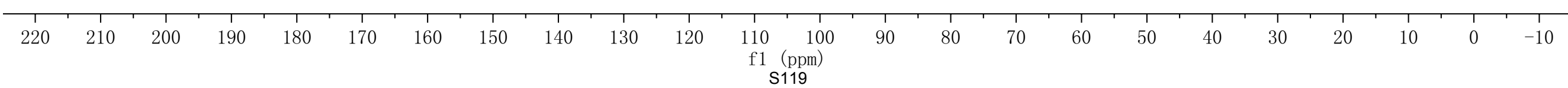




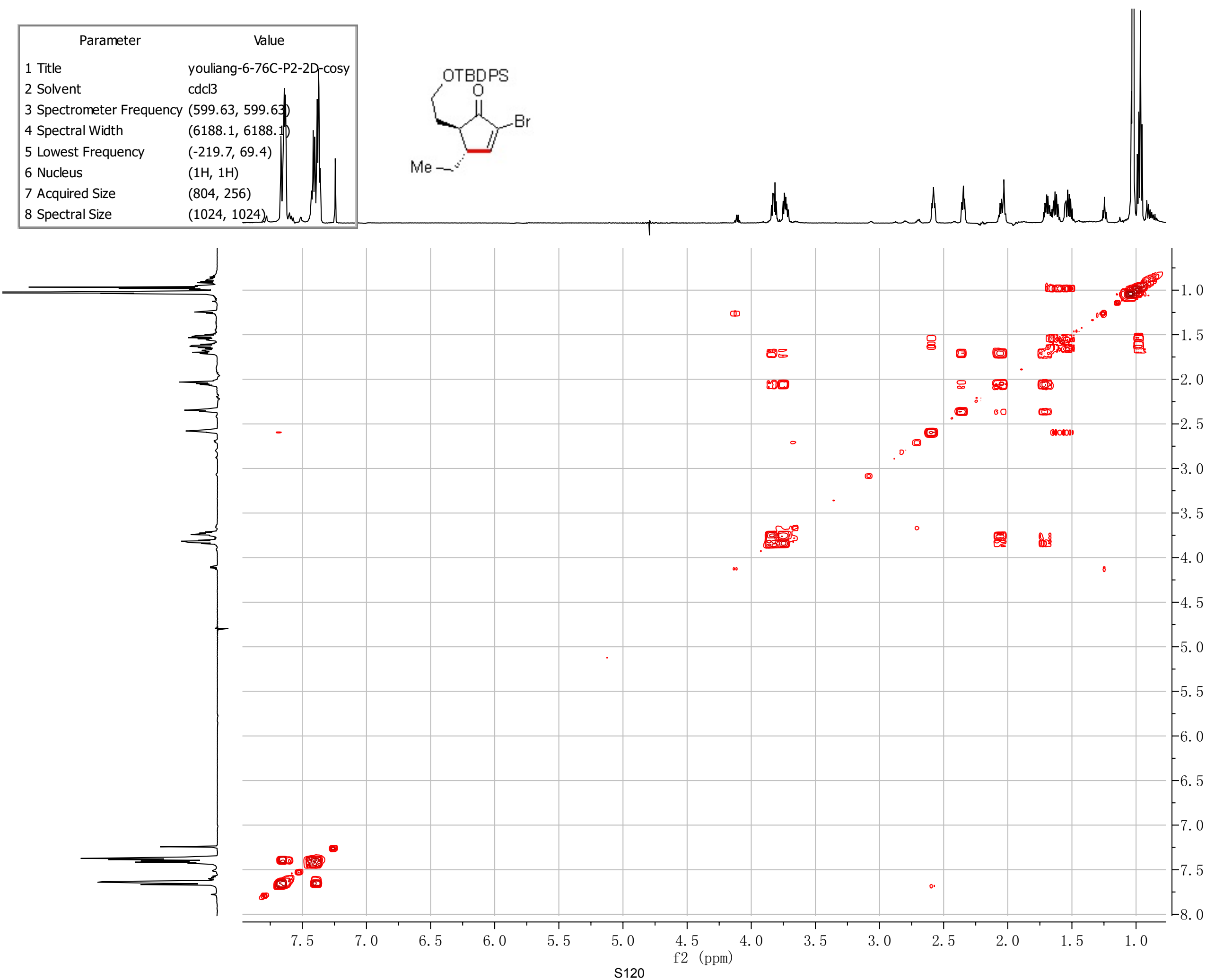




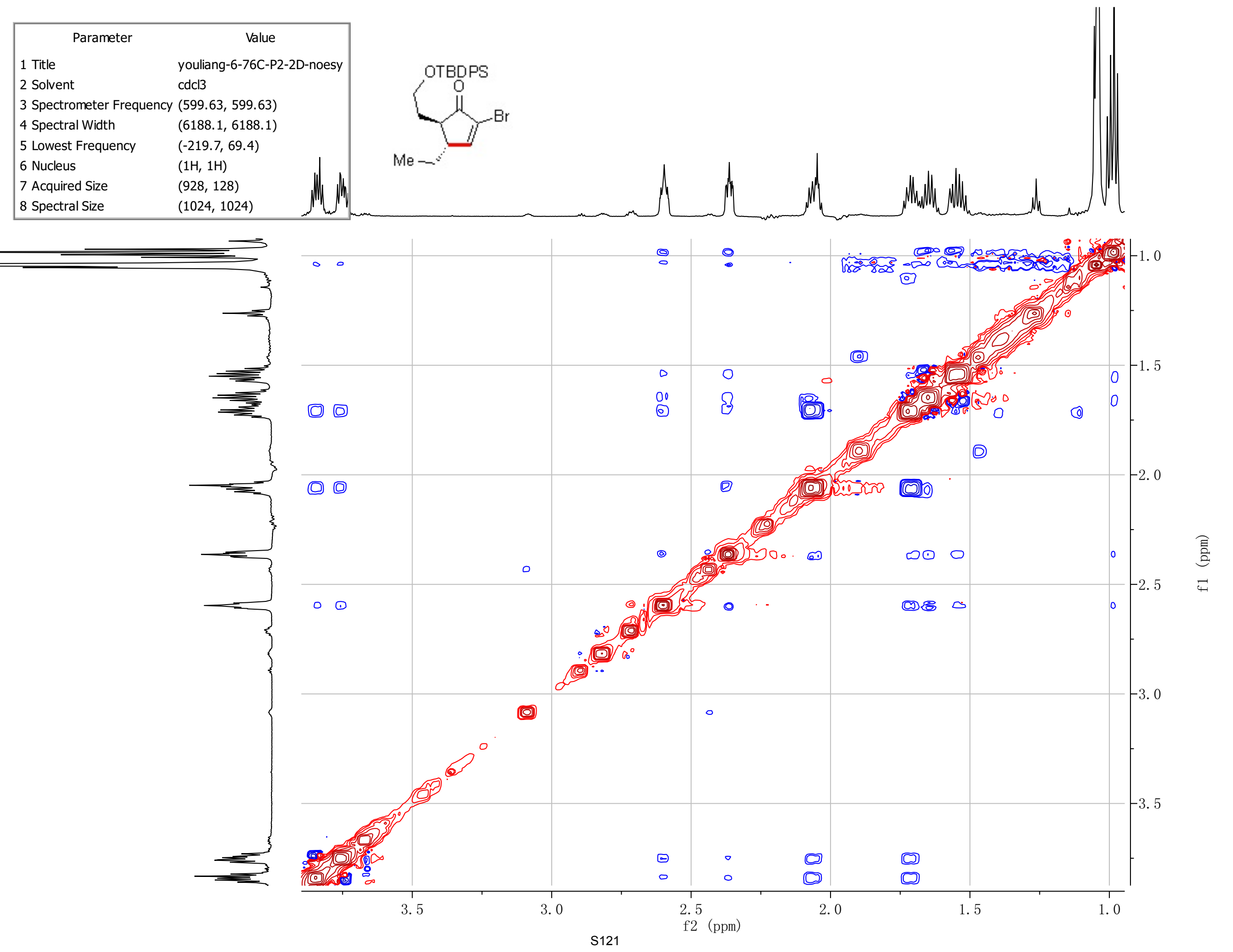




\begin{tabular}{|c|c|}
\hline Parameter & Value \\
\hline 1 Title & youliang-6-74A-again- $P$ \\
\hline 2 Solvent & $\mathrm{CDCl} 3$ \\
\hline 3 Spectrometer Frequency & 499.86 \\
\hline 4 Nucleus & $1 \mathrm{H}$ \\
\hline
\end{tabular}
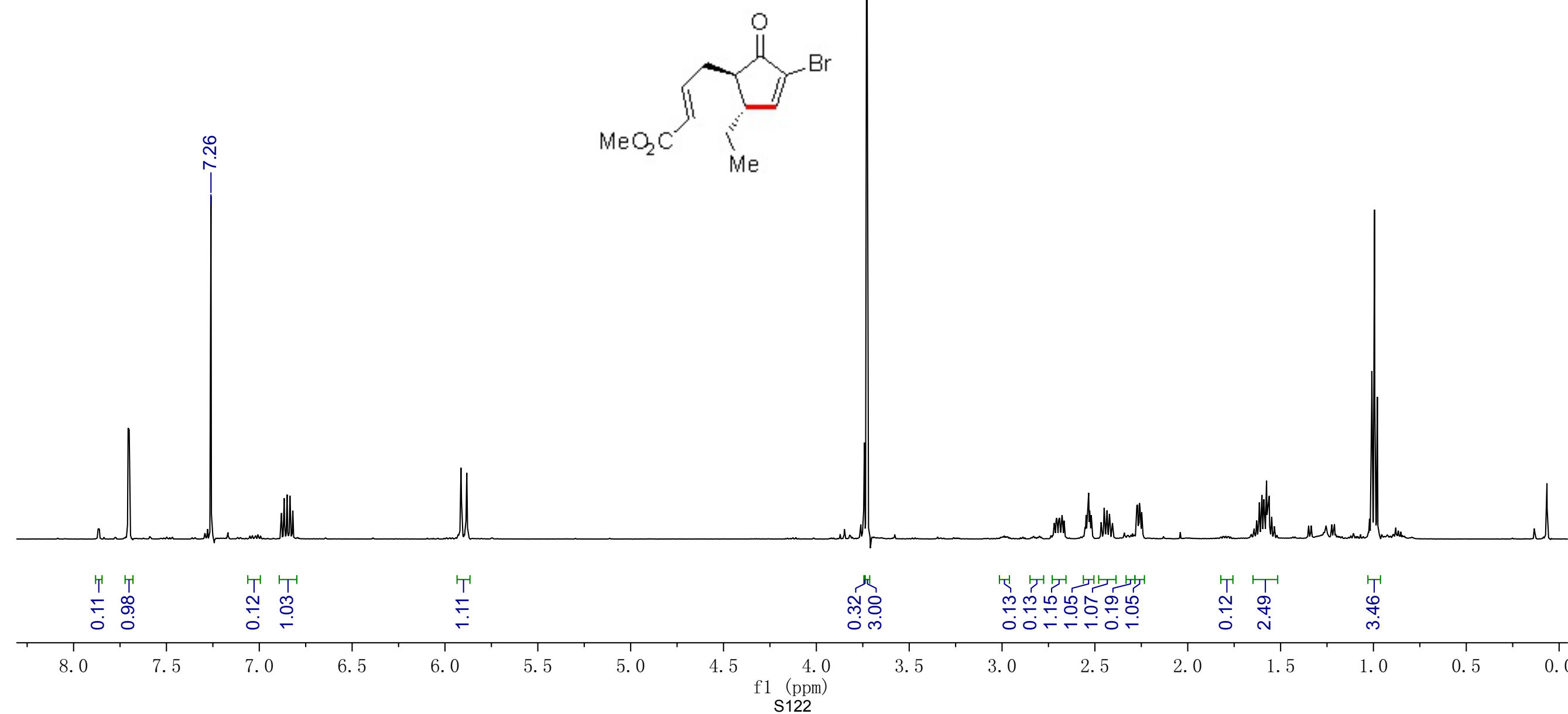


\begin{tabular}{|c|c|}
\hline Parameter & Value \\
\hline 1 Title & youliang-6-74A-again-P-C13e \\
\hline 2 Solvent & $\mathrm{CDCl} 3$ \\
\hline 3 Spectrometer Frequency & 125.70 \\
\hline 4 Nucleus & $13 C$ \\
\hline
\end{tabular}<smiles>CC(C)=CC=CCC1C(=O)C(Br)=CC1CN</smiles>

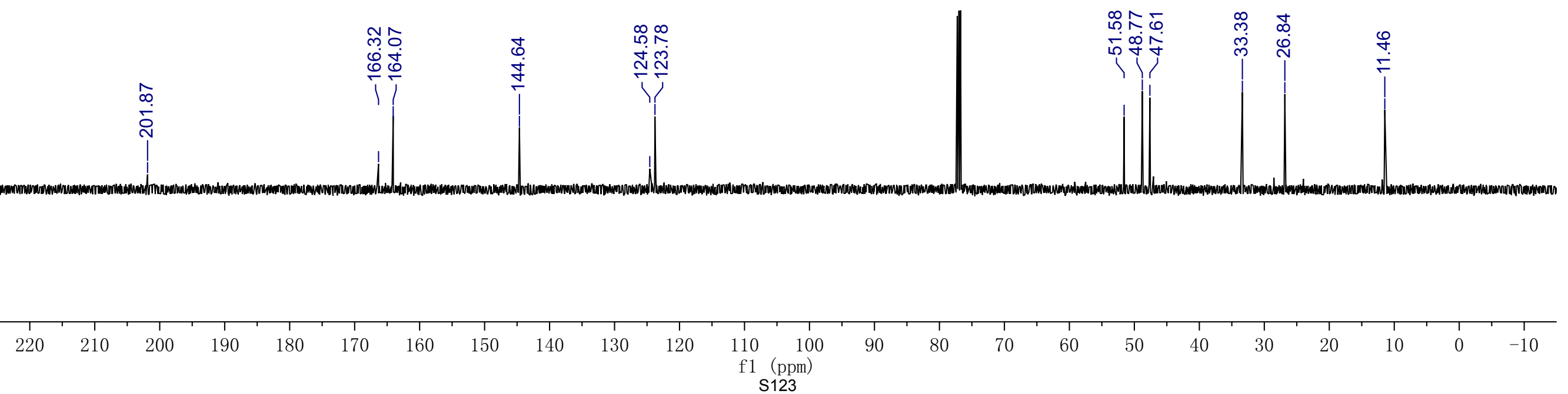




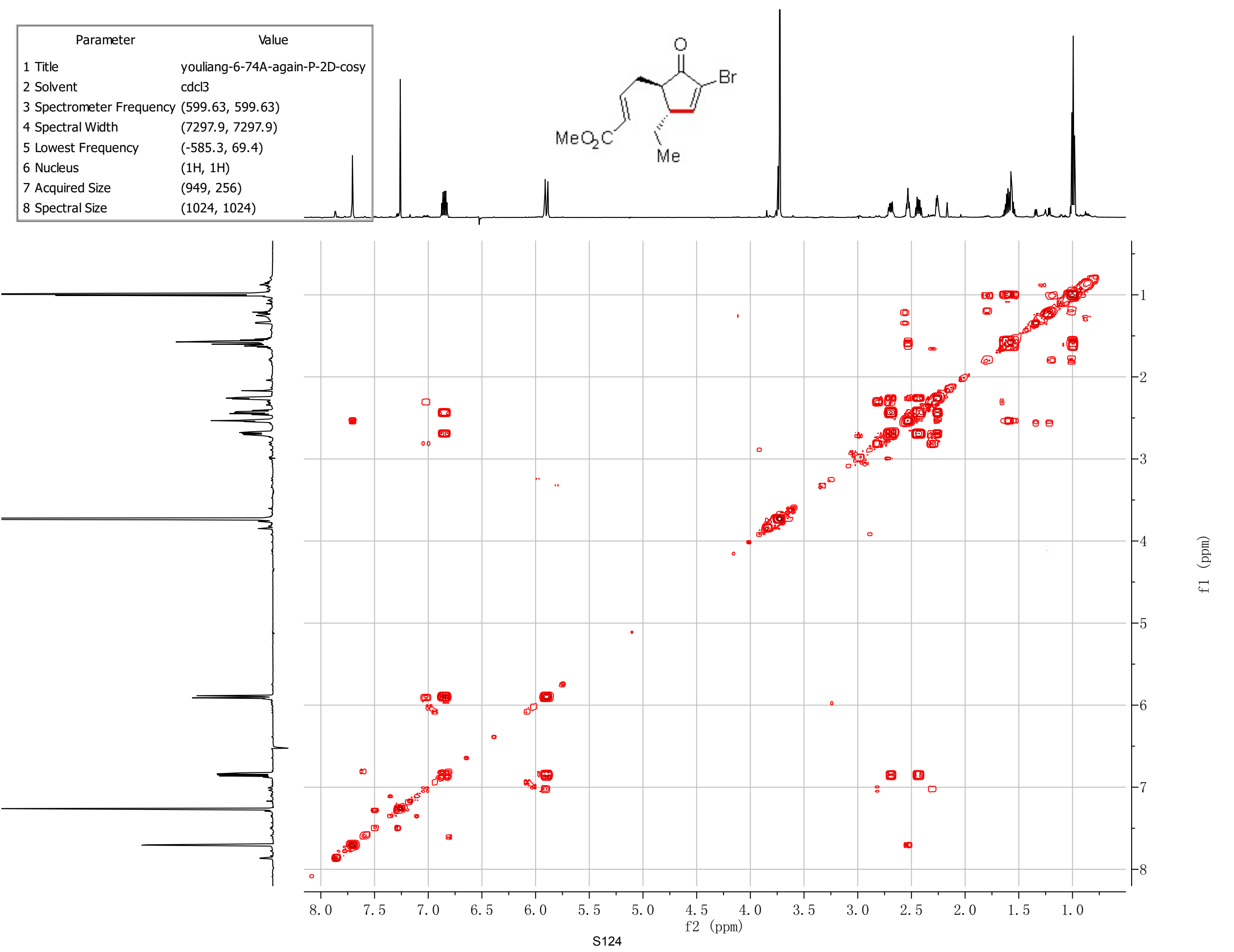




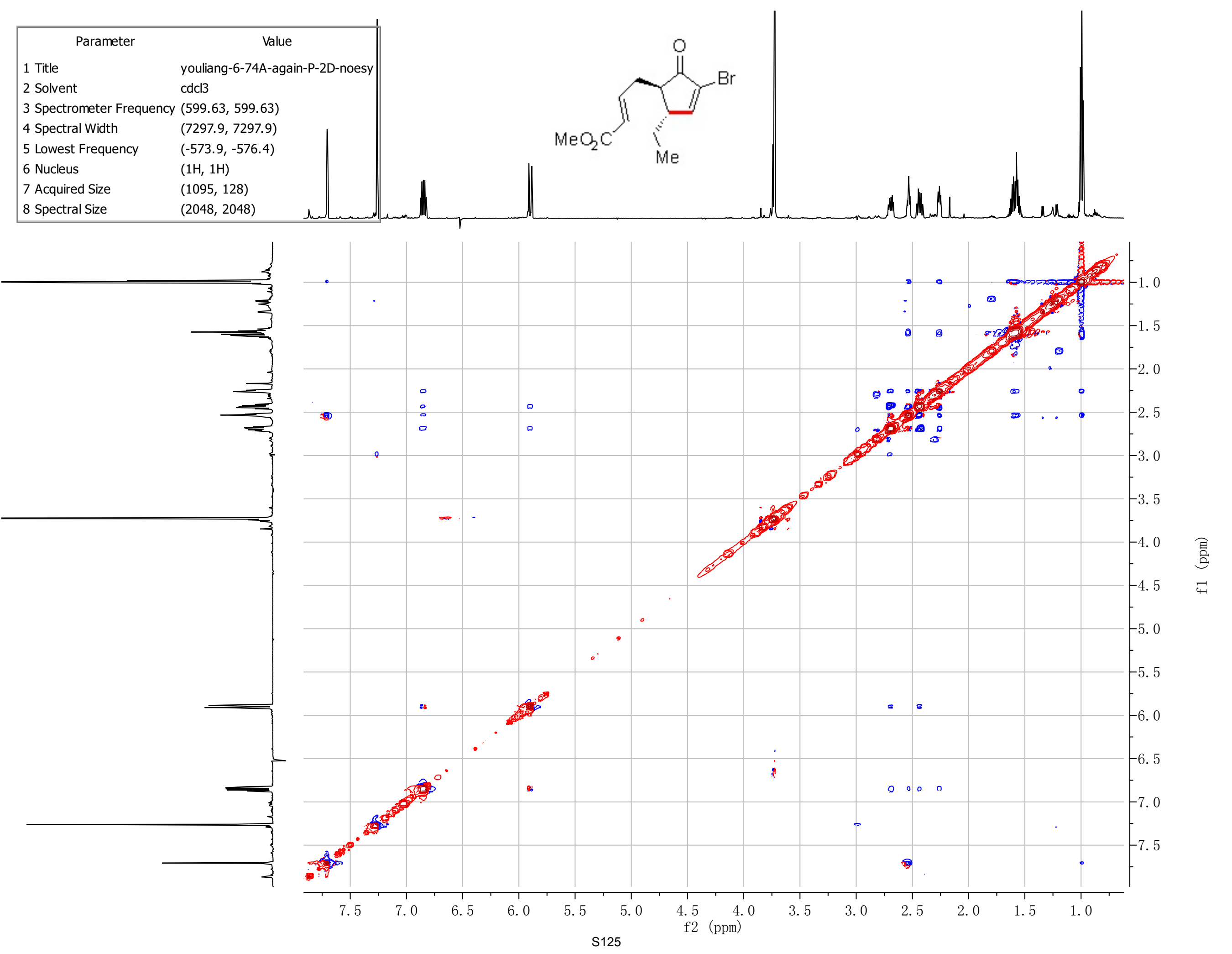




\begin{tabular}{|ll|}
\hline \multicolumn{1}{|c|}{ Parameter } & \multicolumn{1}{c|}{ Value } \\
1 Title & youliang-6-48B-P \\
2 Solvent & CDCl3 \\
3 Spectrometer Frequency & 499.86 \\
4 Nucleus & $1 \mathrm{H}$ \\
\hline
\end{tabular}
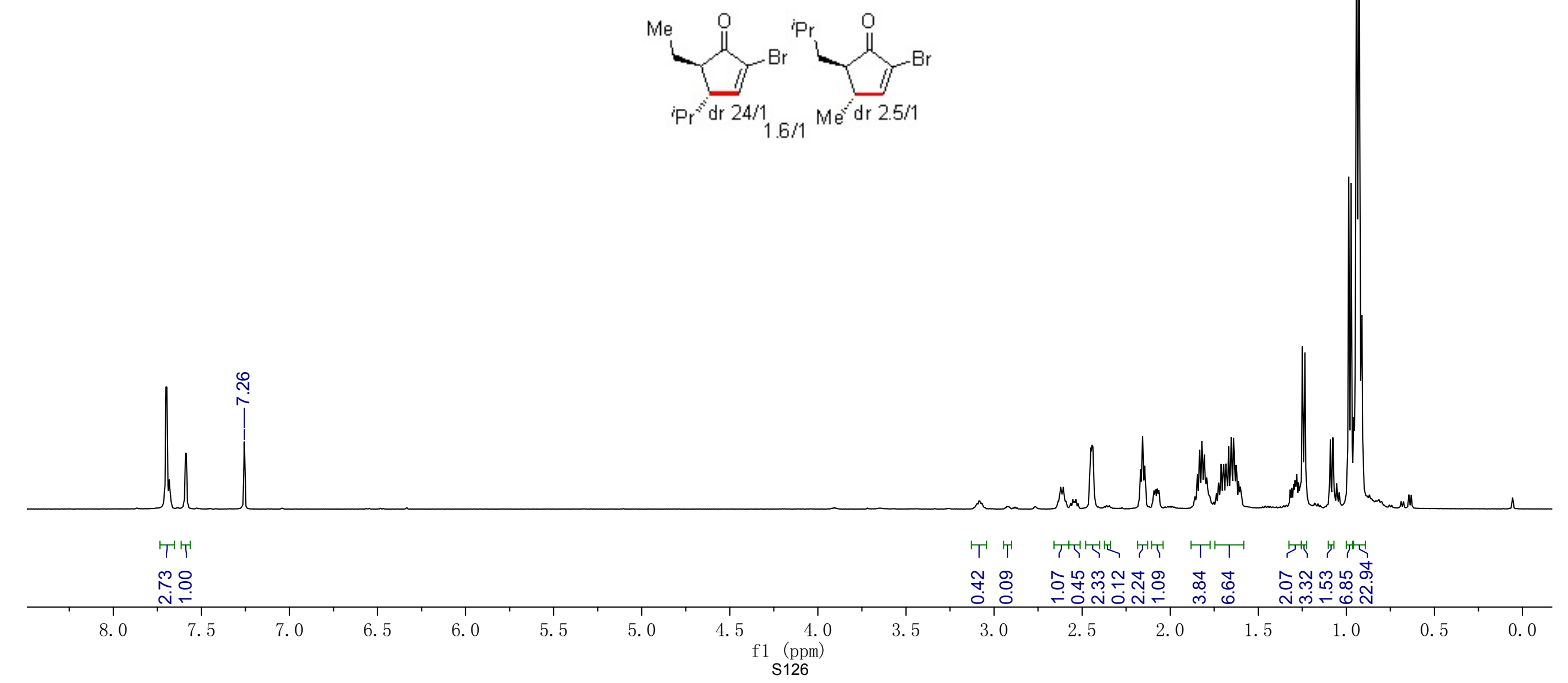


\begin{tabular}{|ll|}
\hline \multicolumn{1}{|c|}{ Parameter } & \multicolumn{1}{c|}{ Value } \\
1 Title & youliang-6-48B-P-C13d \\
2 Solvent & $\mathrm{CDCl} 3$ \\
3 Spectrometer Frequency & 125.70 \\
4 Nucleus & $13 \mathrm{C}$ \\
\hline
\end{tabular}
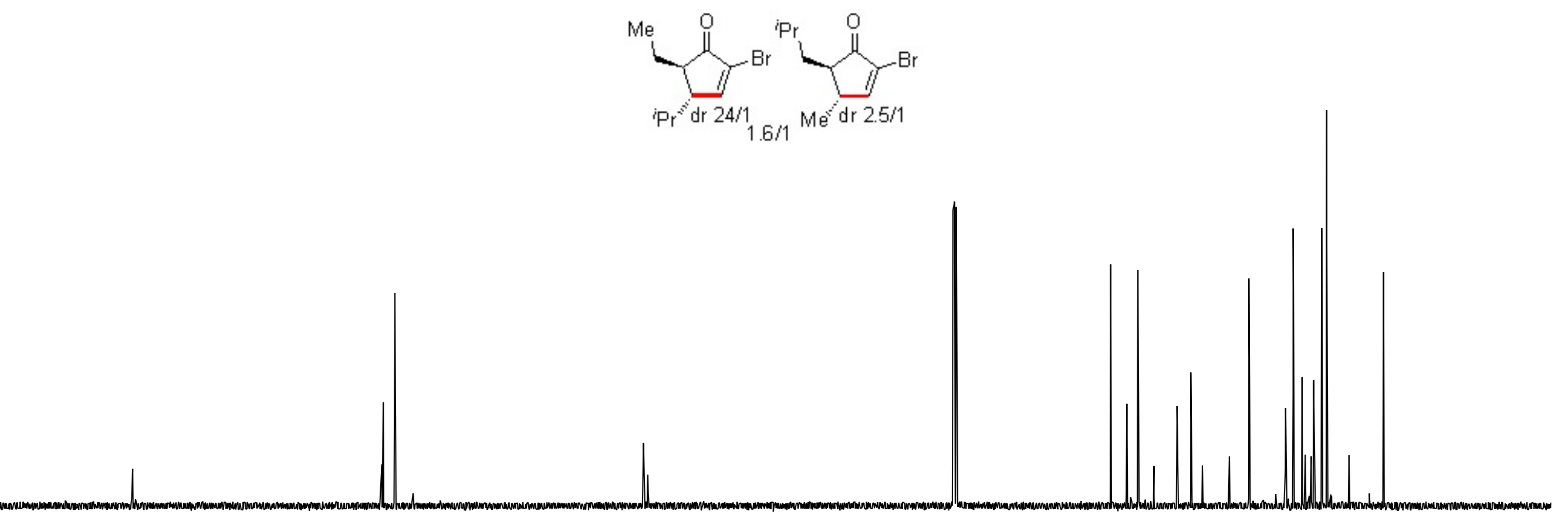

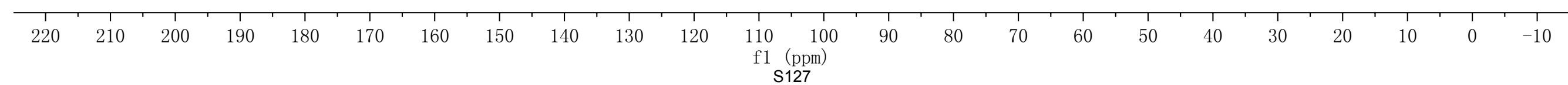




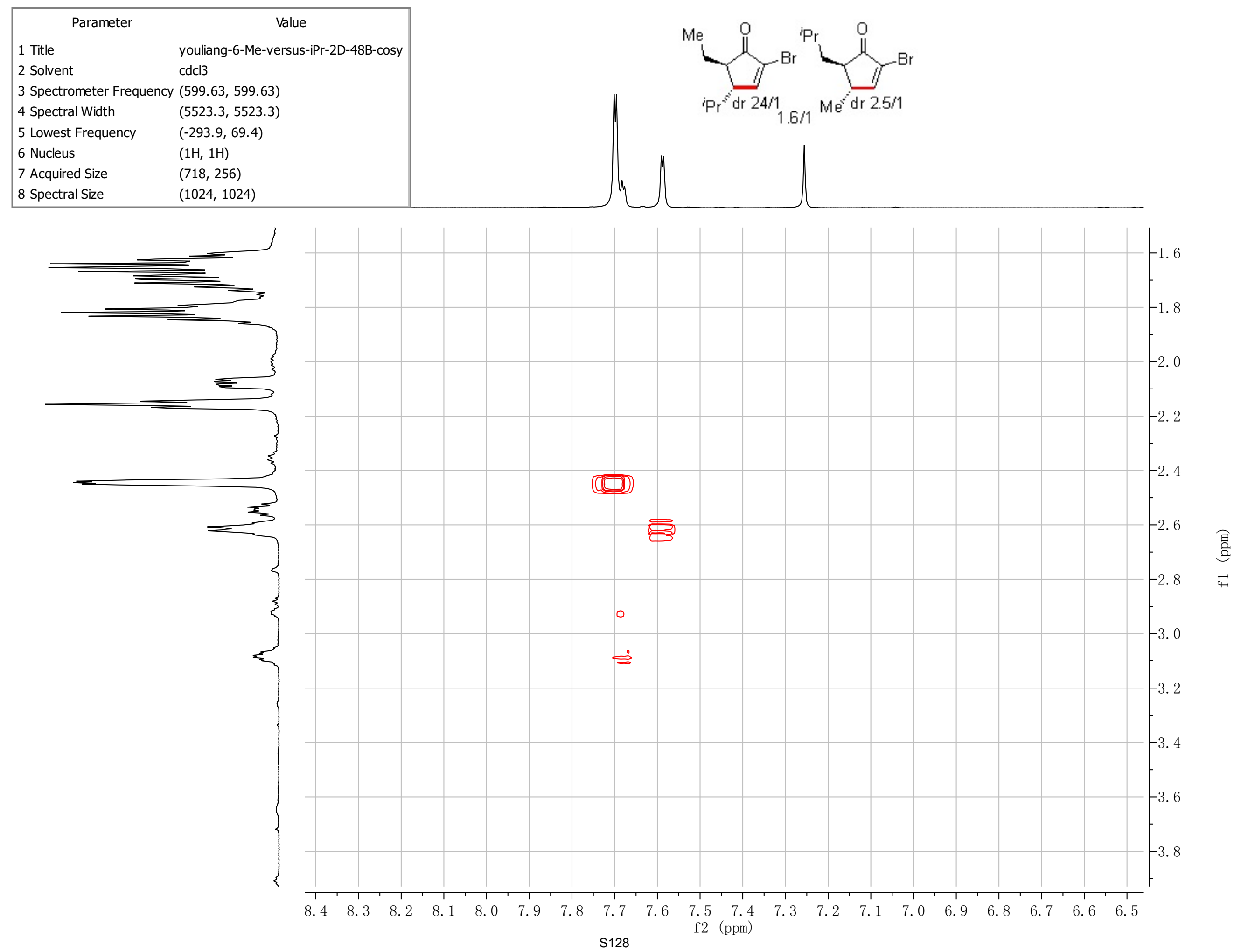




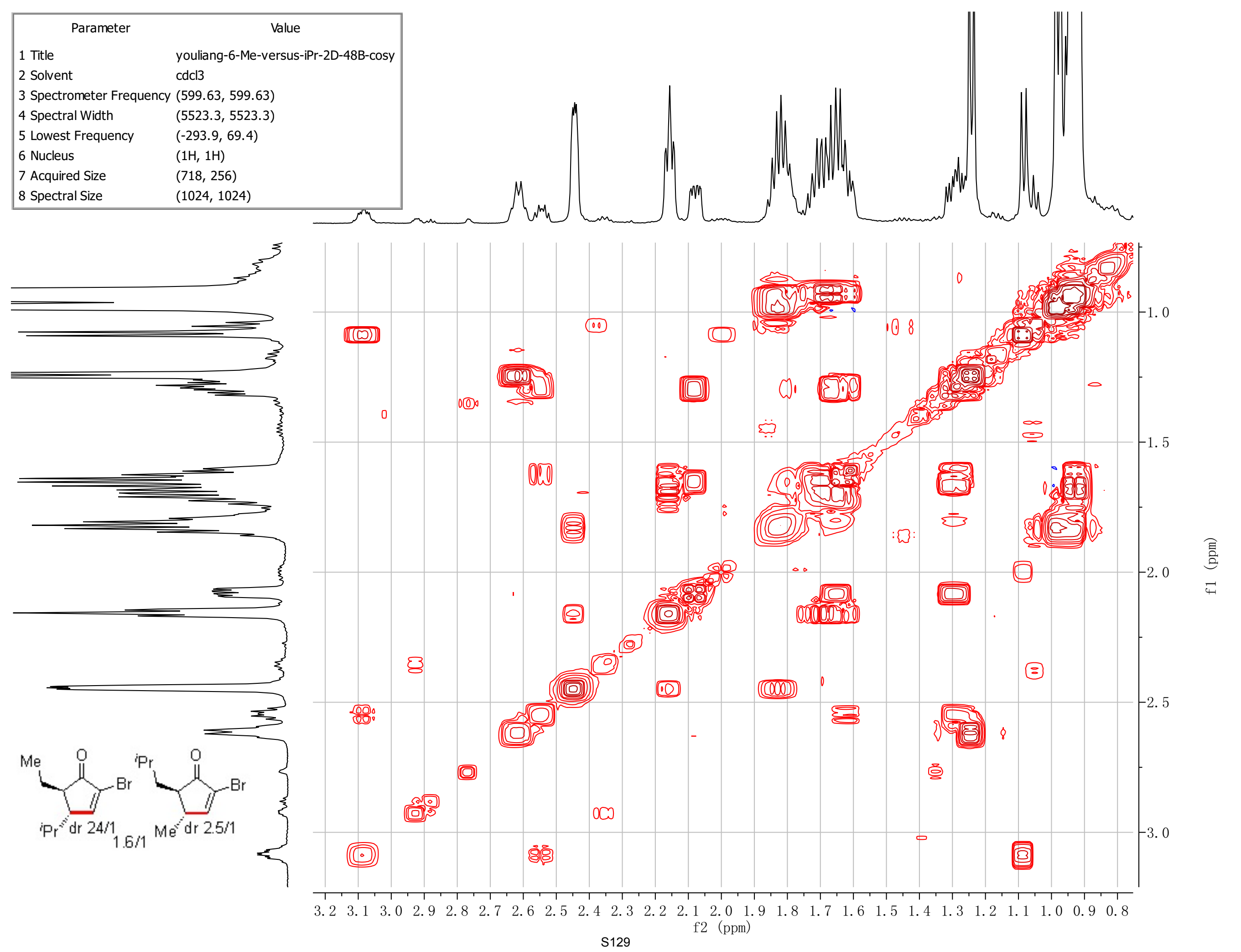




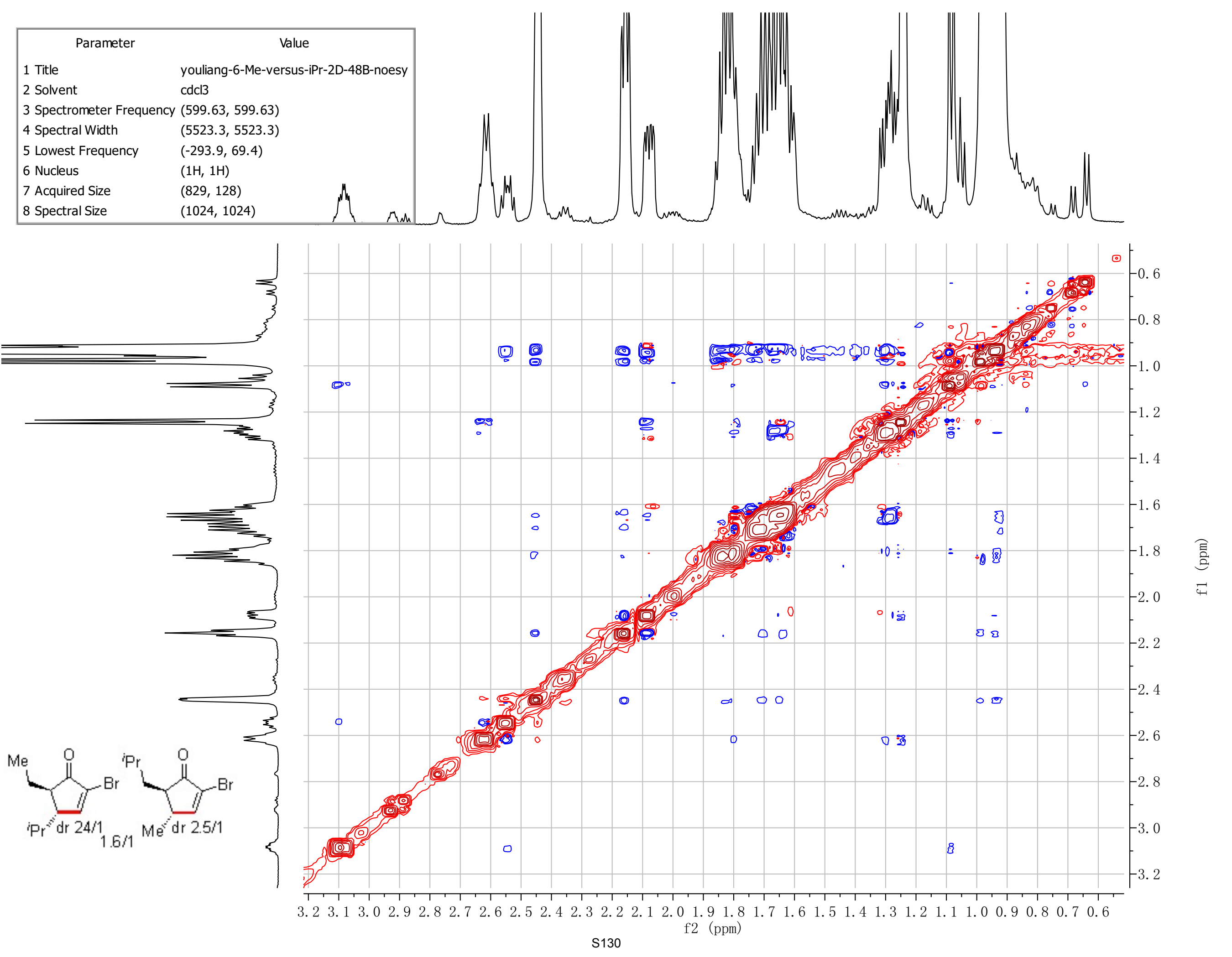




\begin{tabular}{|ll|}
\hline \multicolumn{1}{|c|}{ Parameter } & \multicolumn{1}{c|}{ Value } \\
1 Title & youliang-6-41C1-P \\
2 Solvent & $\mathrm{CDCl3}$ \\
3 Spectrometer Frequency & 499.86 \\
4 Nucleus & $1 \mathrm{H}$ \\
\hline
\end{tabular}
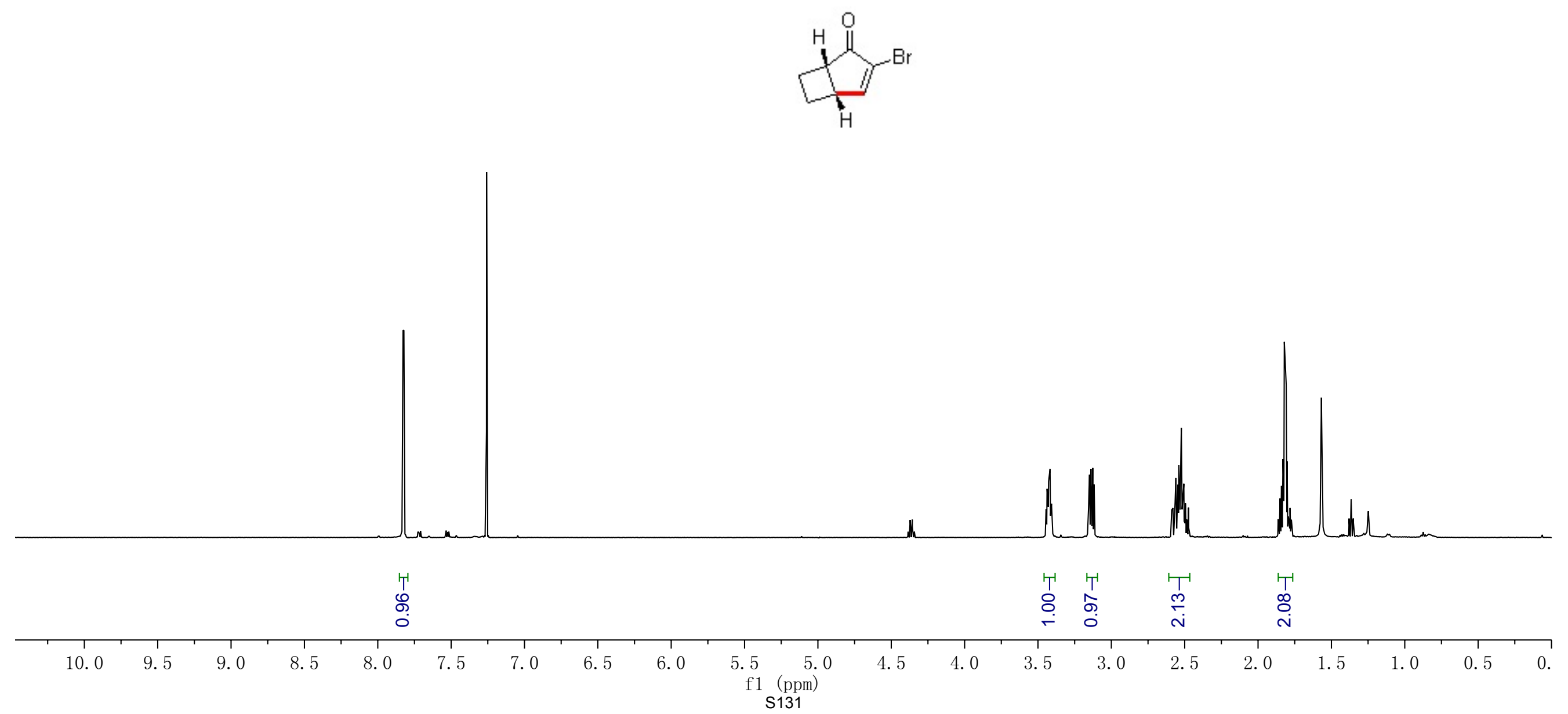
Spectrometer Frequency 125.70
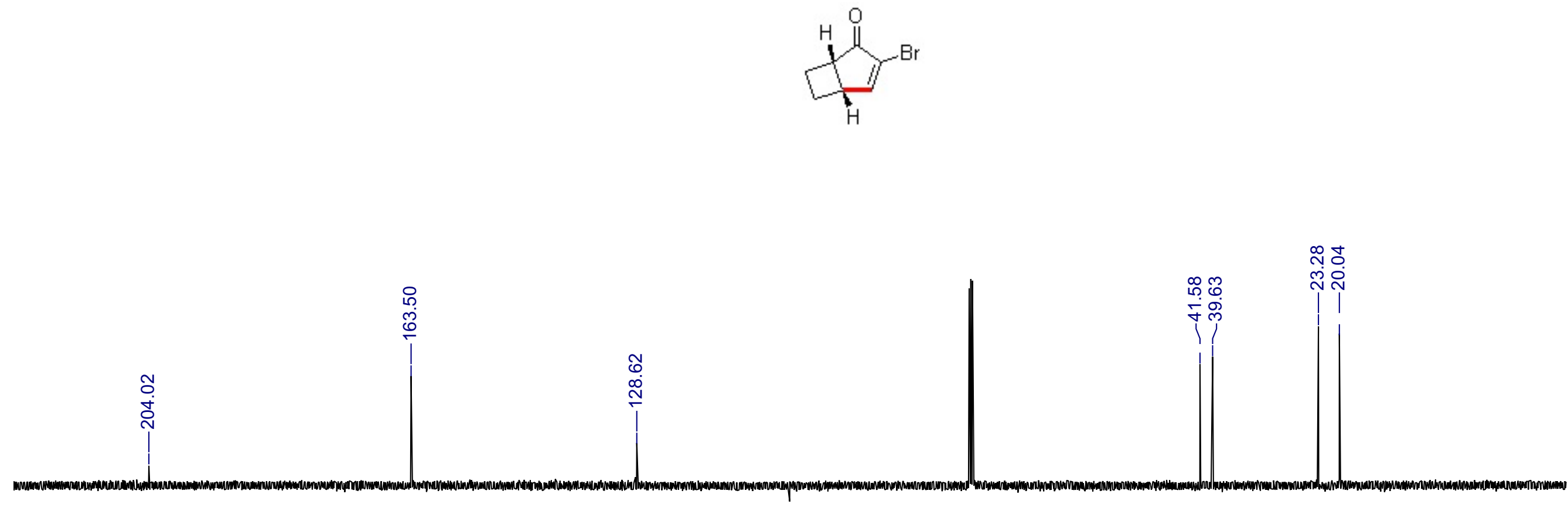

\begin{tabular}{|c|c|c|c|c|c|c|c|c|c|c|c|c|c|c|c|c|c|c|c|c|c|c|c|}
\hline $\begin{array}{c}1 \\
220\end{array}$ & 210 & $\begin{array}{c}1 \\
200\end{array}$ & $\begin{array}{c}19 \\
190\end{array}$ & $\begin{array}{c}1 \\
180\end{array}$ & $\begin{array}{c}1 \\
170\end{array}$ & $\begin{array}{c}1 \\
160\end{array}$ & $\begin{array}{c}1 \\
150\end{array}$ & 140 & 130 & $\begin{array}{c}1 \\
120\end{array}$ & $\begin{array}{r}110 \\
\mathrm{f} 1\end{array}$ & $\begin{array}{l}1 \\
100 \\
\mathrm{pm})\end{array}$ & 90 & 80 & 70 & 60 & $\begin{array}{l}1 \\
50\end{array}$ & 40 & 30 & 20 & 10 & $\begin{array}{l}1 \\
0\end{array}$ & $\begin{array}{c}1 \\
-10\end{array}$ \\
\hline
\end{tabular}




\begin{tabular}{|ll|}
\hline \multicolumn{1}{|c|}{ Parameter } & \multicolumn{1}{c|}{ Value } \\
1 Title & youliang-6-3-A-P \\
2 Solvent & CDCl3 \\
3 Spectrometer Frequency & 499.86 \\
4 Nucleus & $1 \mathrm{H}$ \\
\hline
\end{tabular}
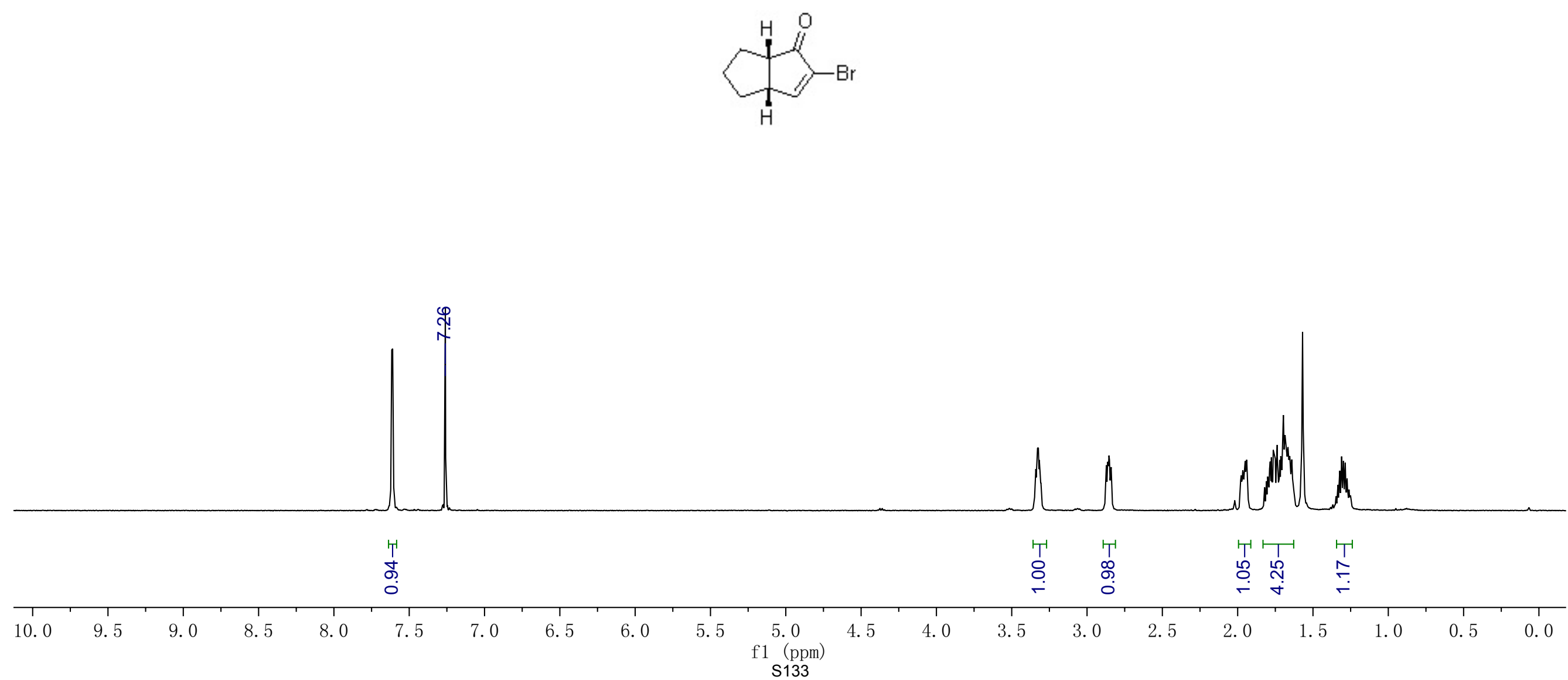


\begin{tabular}{|ll|}
\hline \multicolumn{1}{|c|}{ Parameter } & \multicolumn{1}{c|}{ Value } \\
1 Title & youliang-6-3-A-P-C13k \\
2 Solvent & $\mathrm{CDCl} 3$ \\
3 Spectrometer Frequency & 125.70 \\
4 Nucleus & $13 \mathrm{C}$ \\
\hline
\end{tabular}
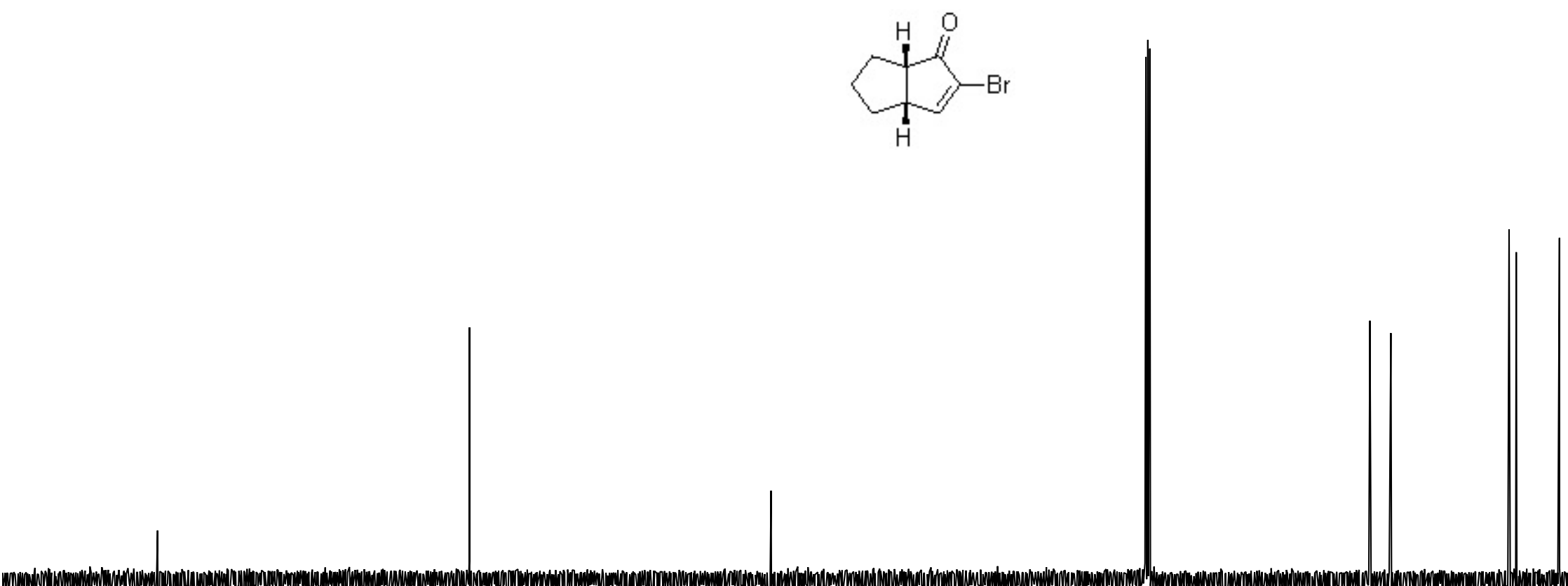

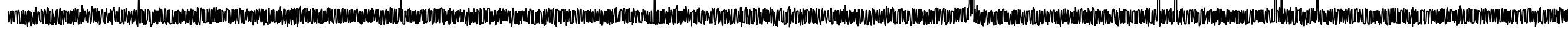

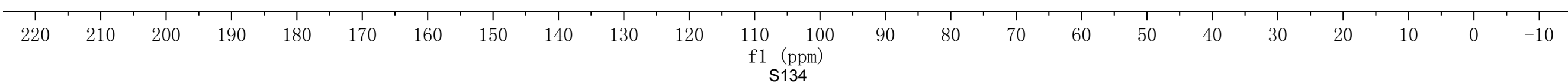



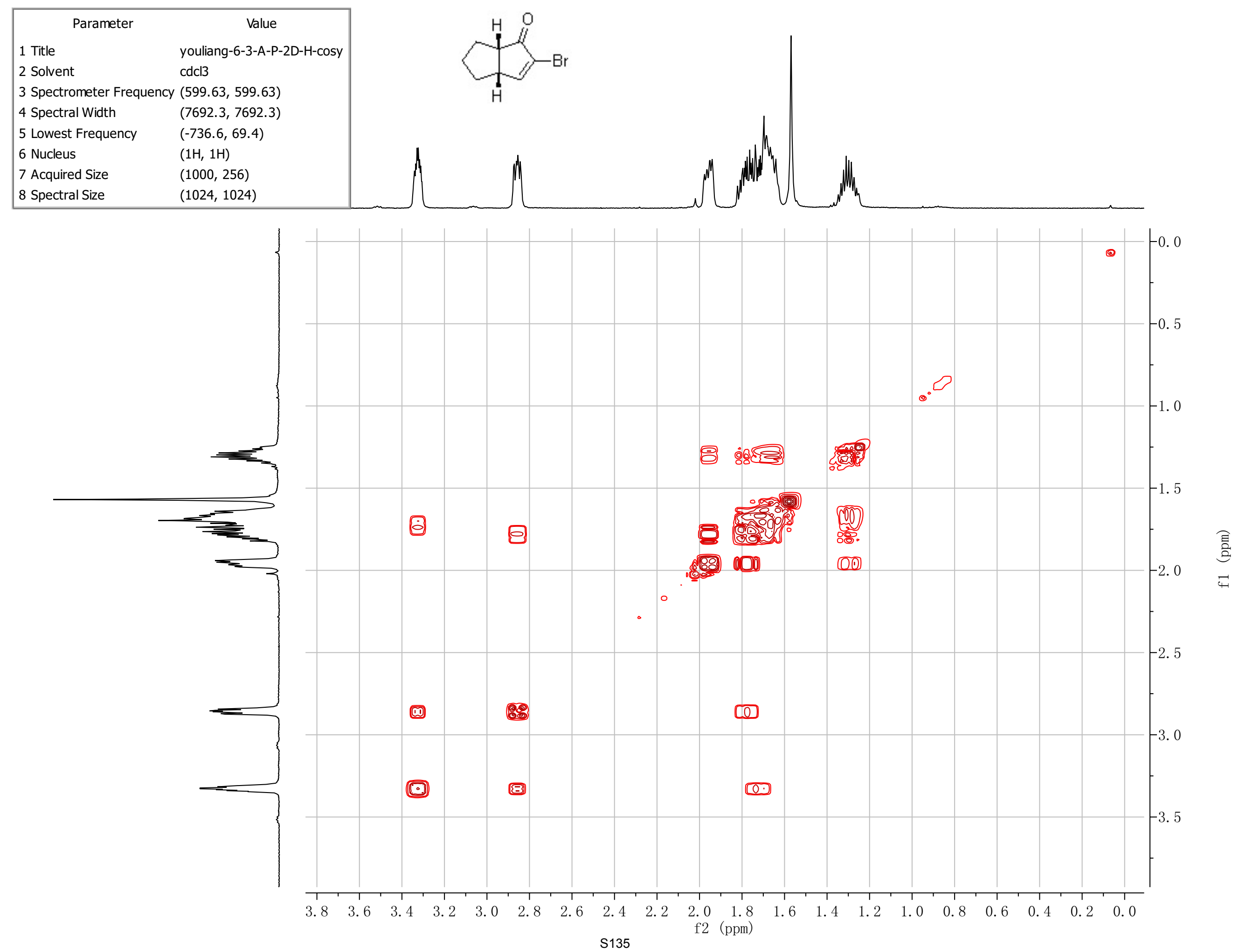


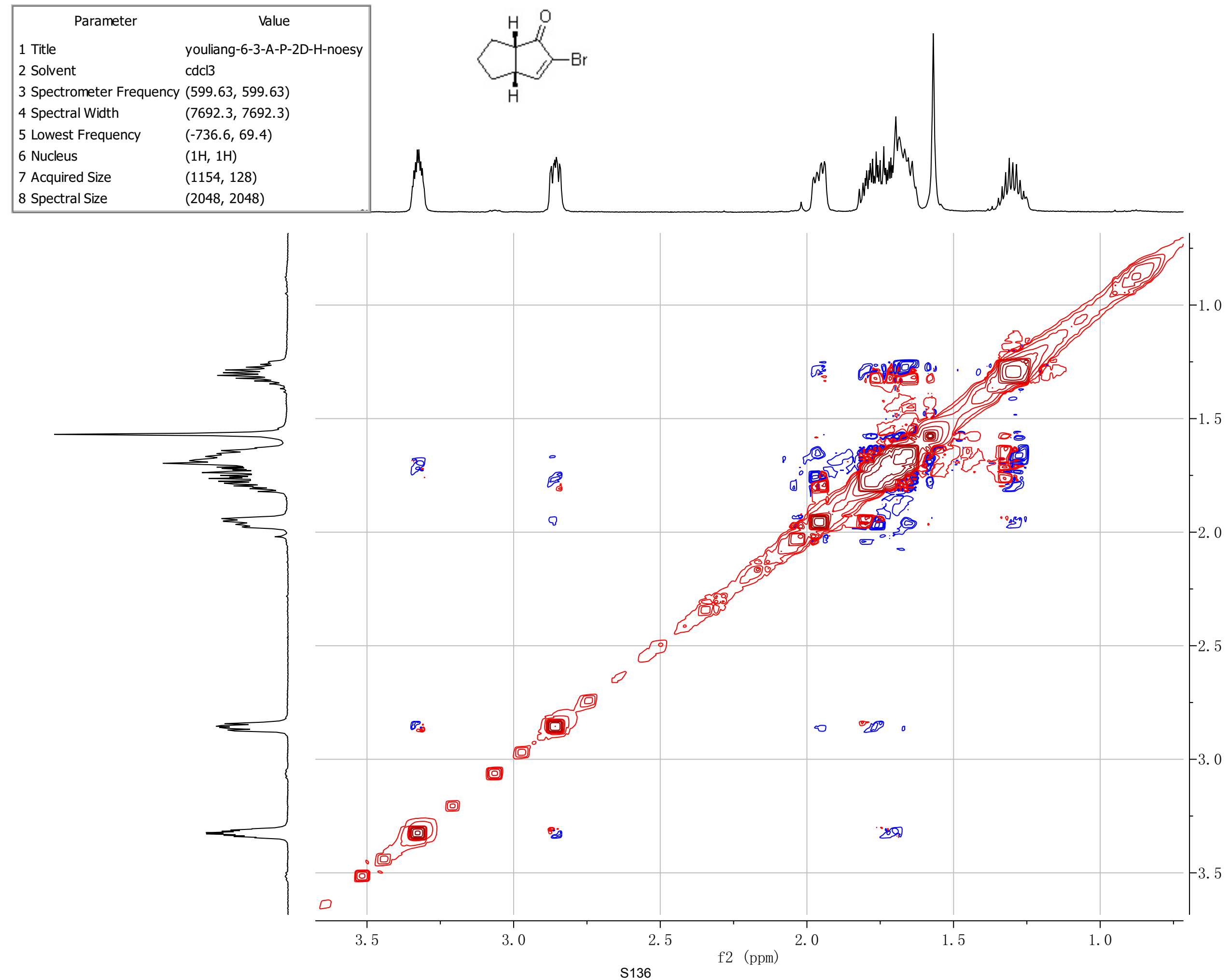




\begin{tabular}{|ll|}
\hline \multicolumn{1}{|c|}{ Parameter } & \multicolumn{1}{c|}{ Value } \\
1 Title & youliang-6-4-A-P \\
2 Solvent & CDCl3 \\
3 Spectrometer Frequency & 499.86 \\
4 Nucleus & $1 \mathrm{H}$ \\
\hline
\end{tabular}

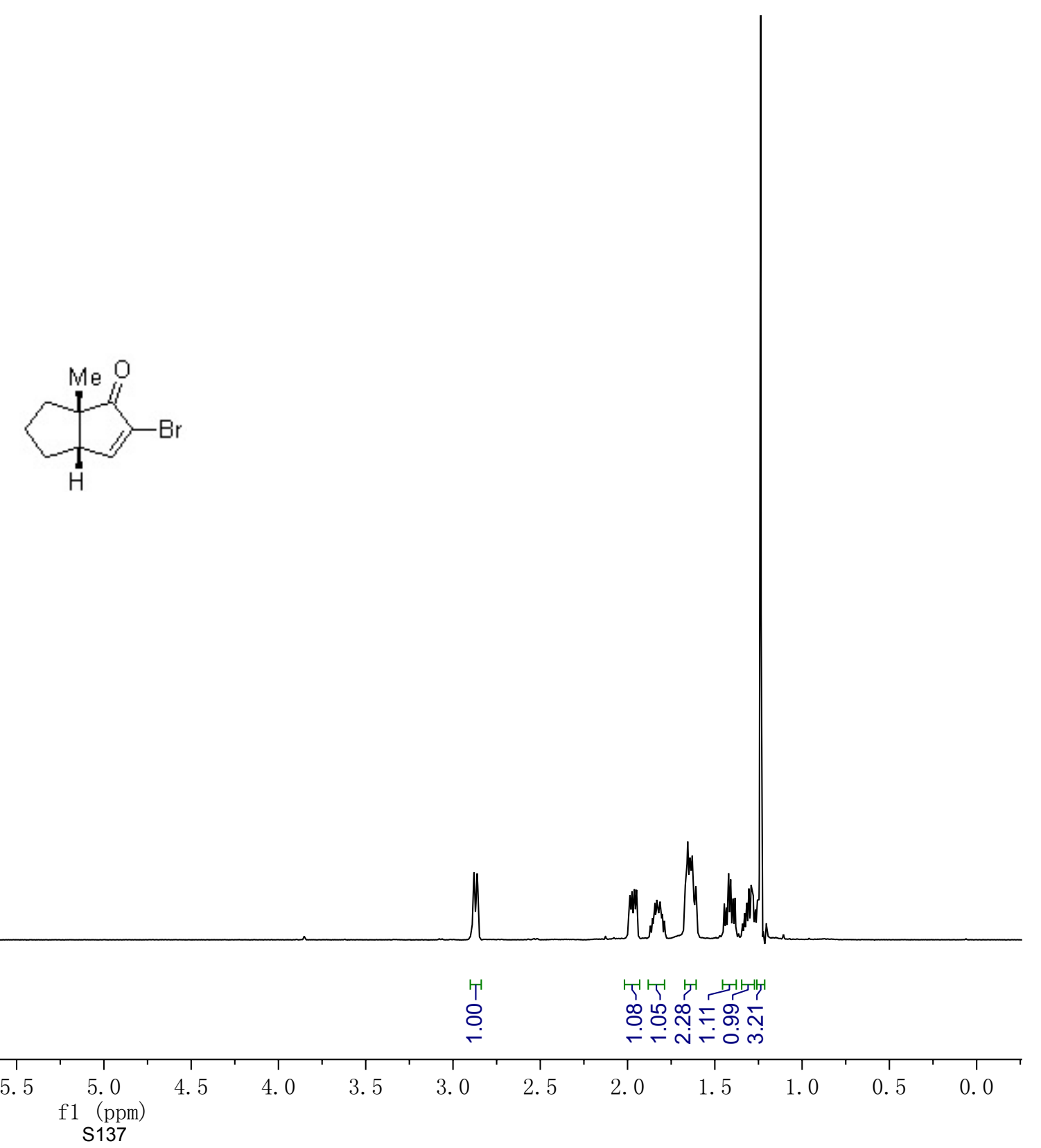




\begin{tabular}{|ll|}
\hline \multicolumn{1}{|c|}{ Parameter } & \multicolumn{1}{c|}{ Value } \\
1 Title & youliang-6-4-A-P-C13g \\
2 Solvent & $\mathrm{CDCl} 3$ \\
3 Spectrometer Frequency & 125.70 \\
4 Nucleus & $13 \mathrm{C}$ \\
\hline
\end{tabular}
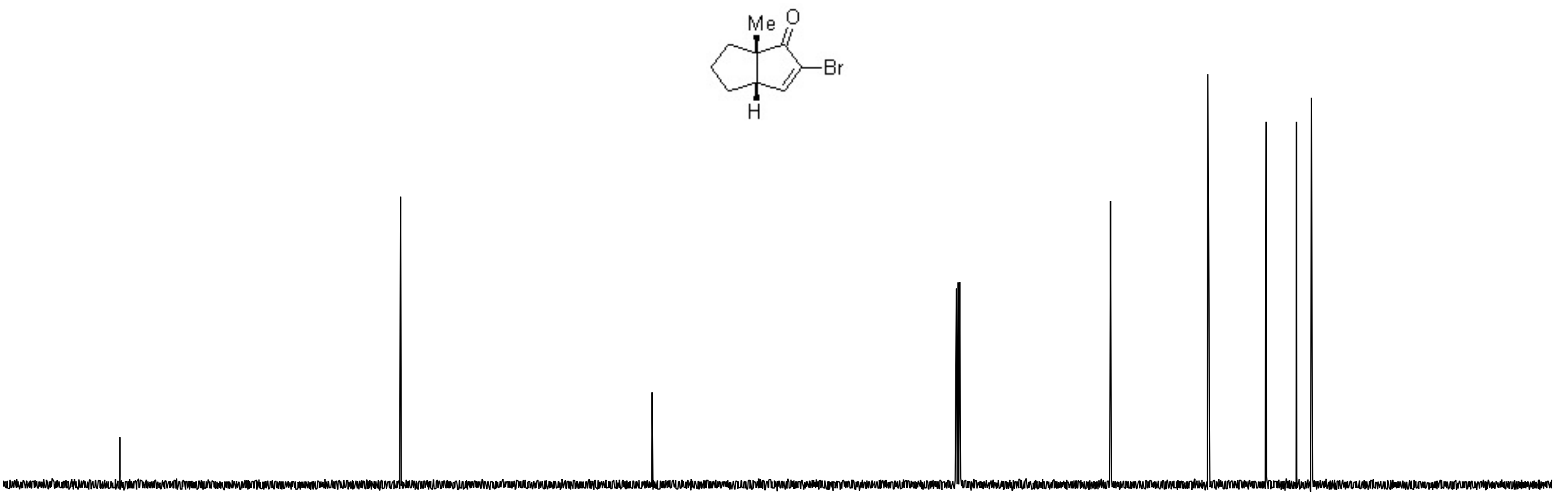

\begin{tabular}{|c|c|c|c|c|c|c|c|c|c|c|c|c|c|c|c|c|c|c|c|c|c|c|c|}
\hline $\begin{array}{c}1 \\
220\end{array}$ & 210 & $\begin{array}{c}1 \\
200\end{array}$ & $\begin{array}{c}1 \\
190\end{array}$ & $\begin{array}{c}180 \\
180\end{array}$ & $\begin{array}{c}1 \\
170\end{array}$ & 160 & $\begin{array}{c}1 \\
150\end{array}$ & 140 & 130 & 120 & $\begin{array}{r}110 \\
\mathrm{f} 1 \\
\mathrm{~S}\end{array}$ & $\begin{array}{l}100 \\
\text { ppm) } \\
38\end{array}$ & $\begin{array}{c}1 \\
90\end{array}$ & $\begin{array}{c}1 \\
80\end{array}$ & $\begin{array}{c}1 \\
70\end{array}$ & $\begin{array}{c}1 \\
60\end{array}$ & $\begin{array}{c}1 \\
50\end{array}$ & $\begin{array}{c}1 \\
40\end{array}$ & 30 & $\begin{array}{c}1 \\
20\end{array}$ & 10 & $\begin{array}{l}1 \\
0\end{array}$ & $\begin{array}{c}1 \\
-10\end{array}$ \\
\hline
\end{tabular}




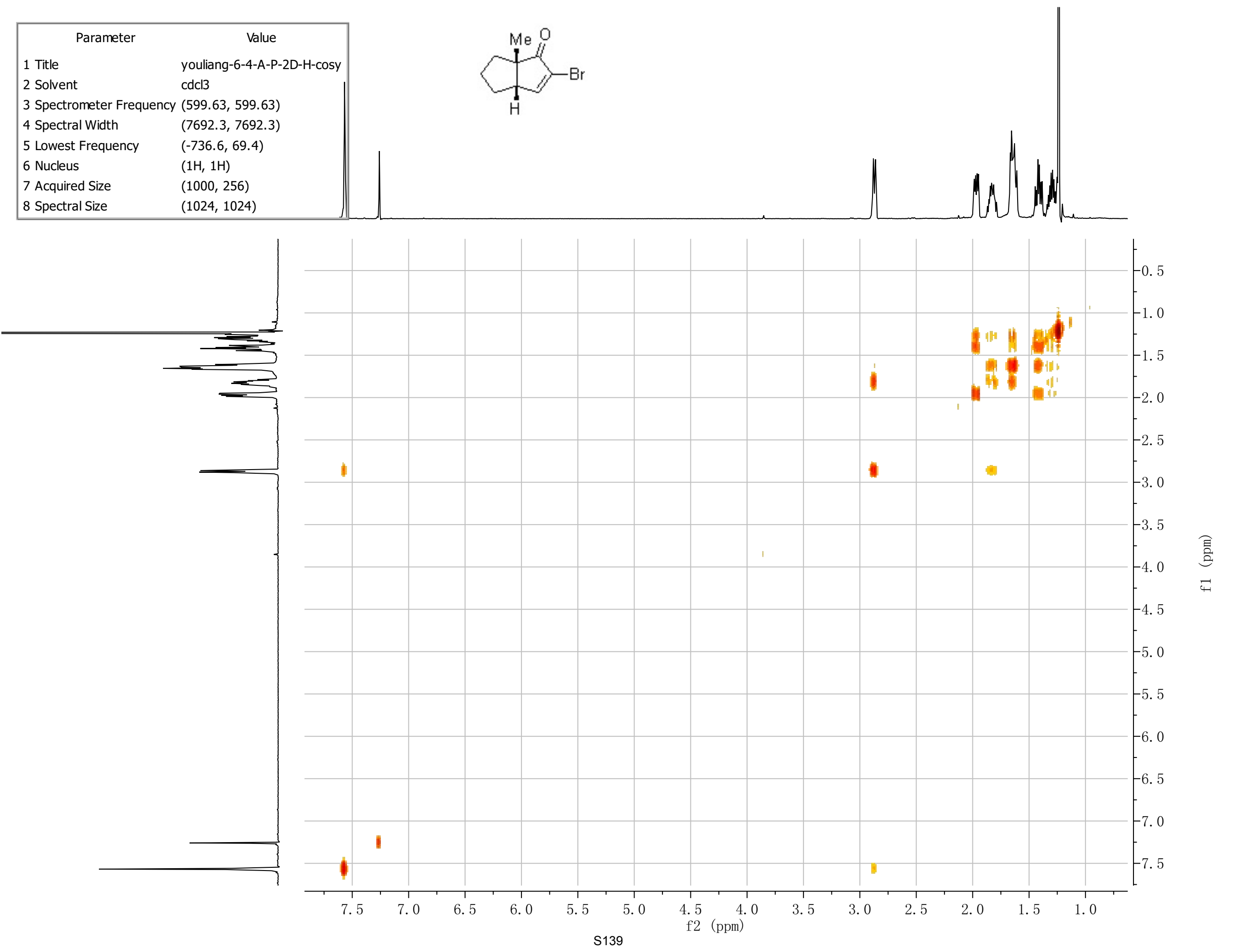




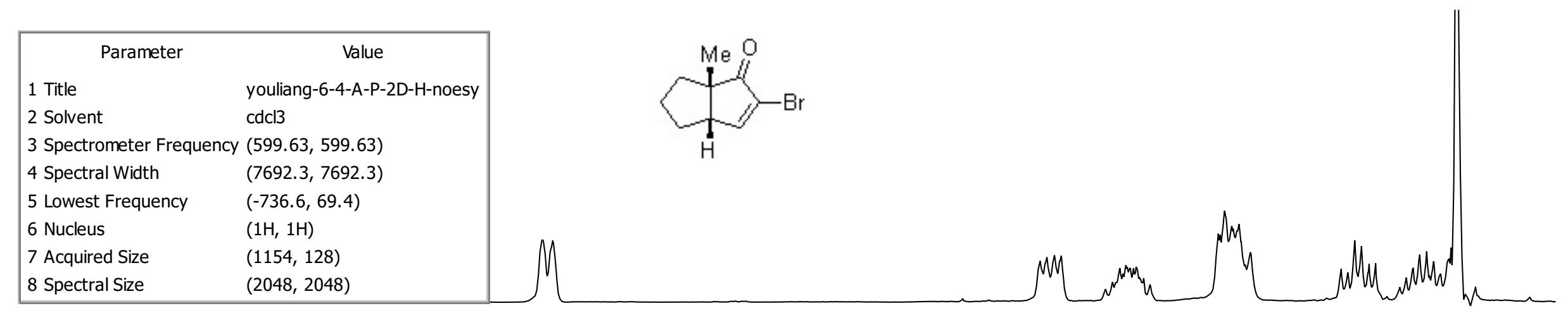

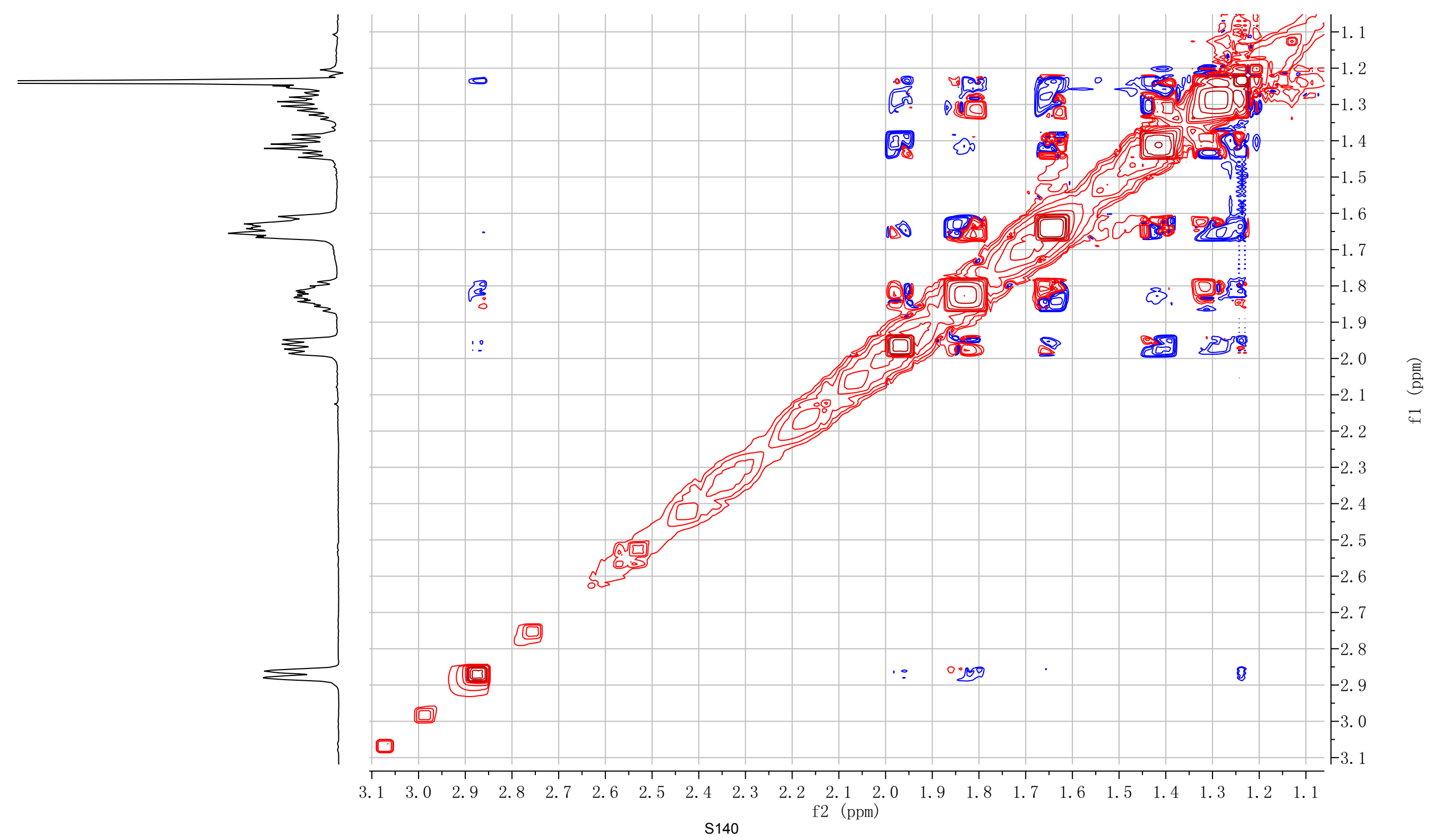




\begin{tabular}{|ll|}
\hline \multicolumn{1}{|c|}{ Parameter } & \multicolumn{1}{c|}{ Value } \\
1 Title & youliang-5-299-C-P \\
2 Solvent & cdc13 \\
3 Spectrometer Frequency & 599.64 \\
4 Nucleus & $1 \mathrm{H}$ \\
\hline
\end{tabular}
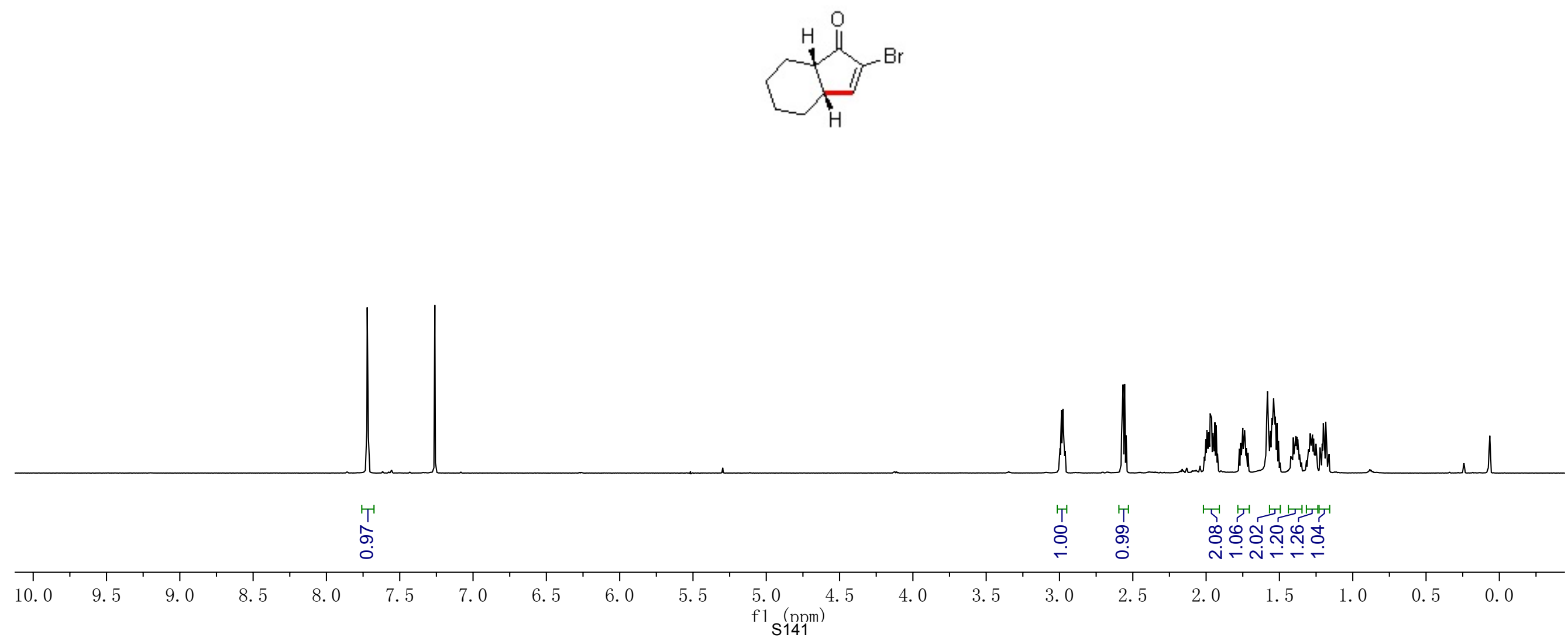


\begin{tabular}{|ll|}
\hline \multicolumn{1}{|c|}{ Parameter } & \multicolumn{1}{c|}{ Value } \\
1 Title & youliang-5-299-C-P-C13I \\
2 Solvent & $\mathrm{CDCl} 3$ \\
3 Spectrometer Frequency & 125.70 \\
4 Nucleus & $13 \mathrm{C}$ \\
\hline
\end{tabular}
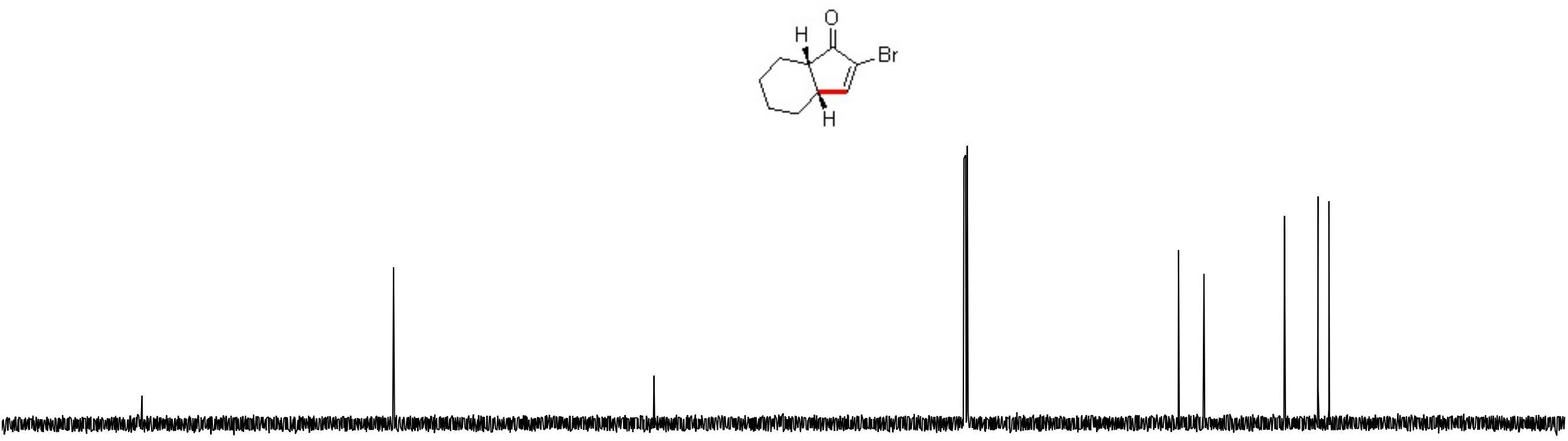

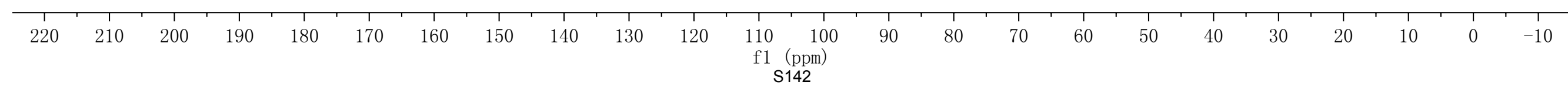




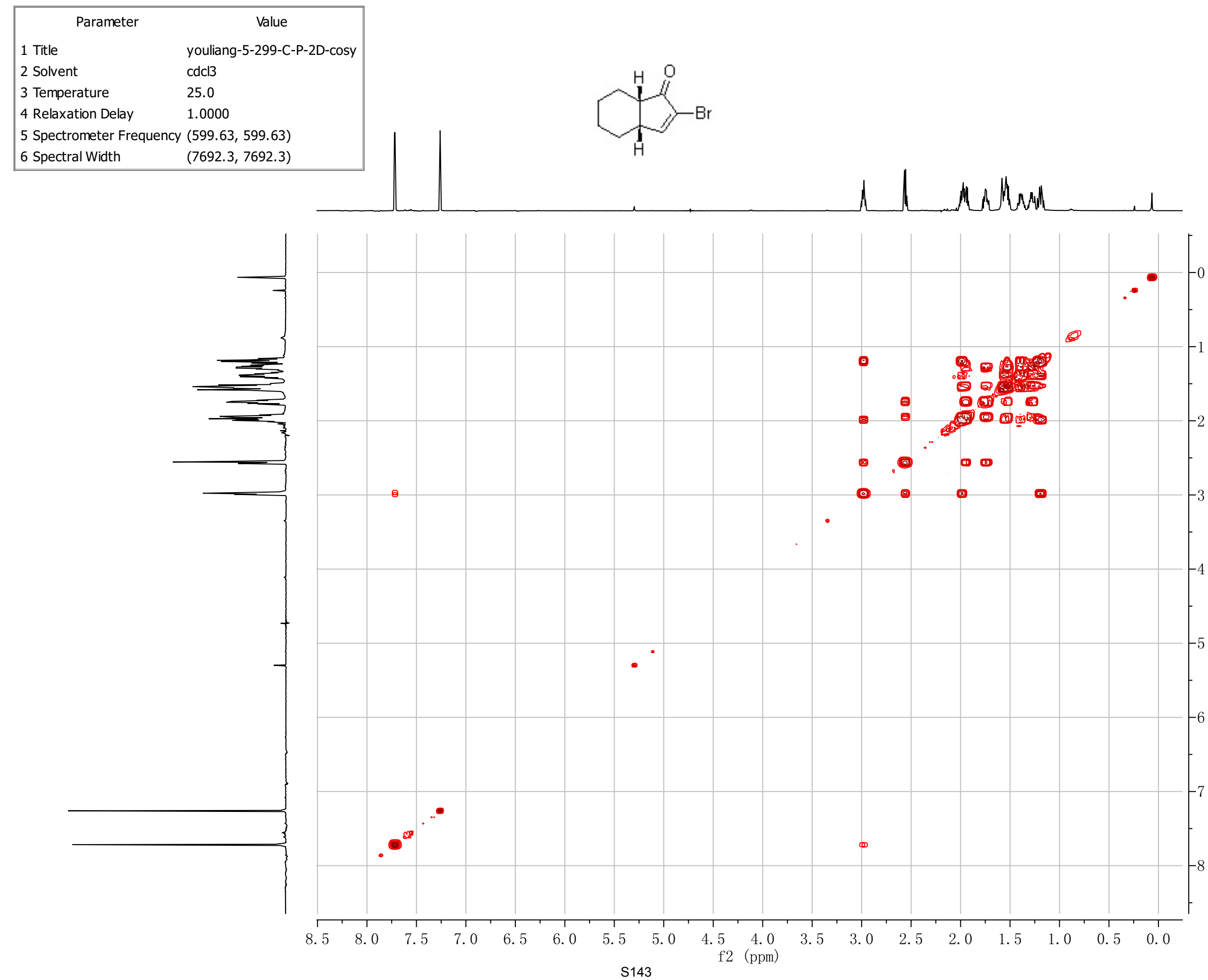




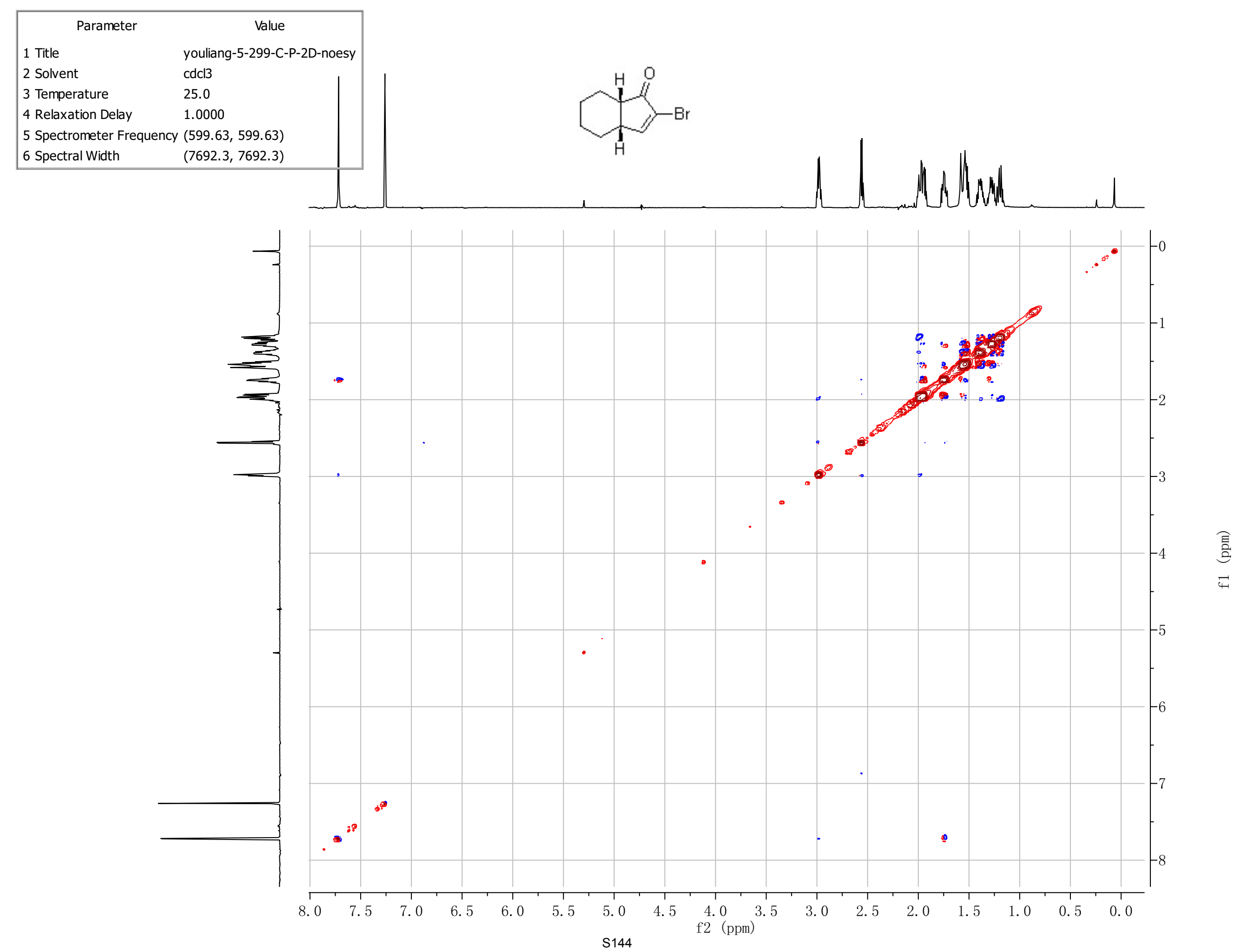




\begin{tabular}{|ll|}
\hline \multicolumn{1}{|c|}{ Parameter } & \multicolumn{1}{c|}{ Value } \\
1 Title & youliang-5-299-B-P \\
2 Solvent & $\mathrm{CDCl3}$ \\
3 Spectrometer Frequency & 499.86 \\
4 Nucleus & $1 \mathrm{H}$ \\
\hline
\end{tabular}
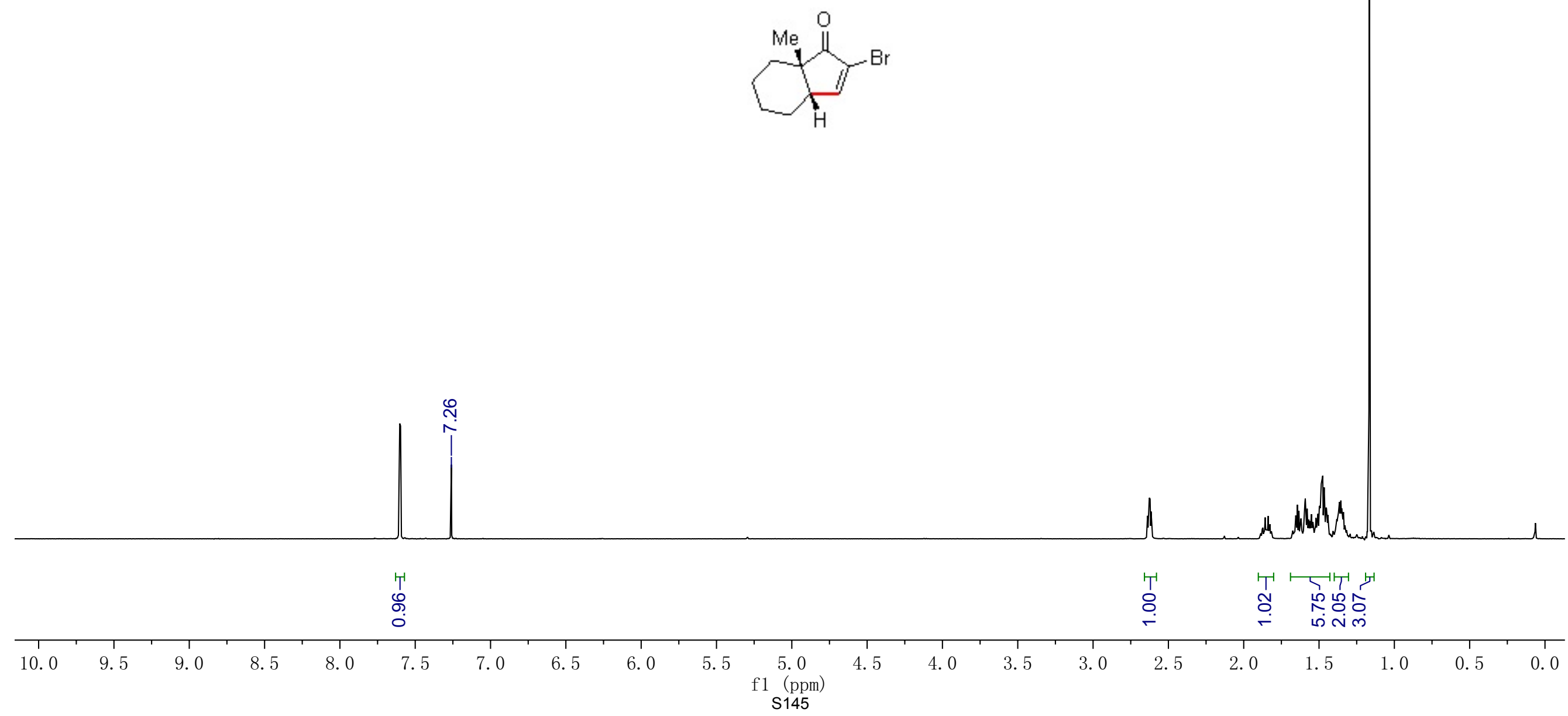
Spectrometer Frequency 125.70
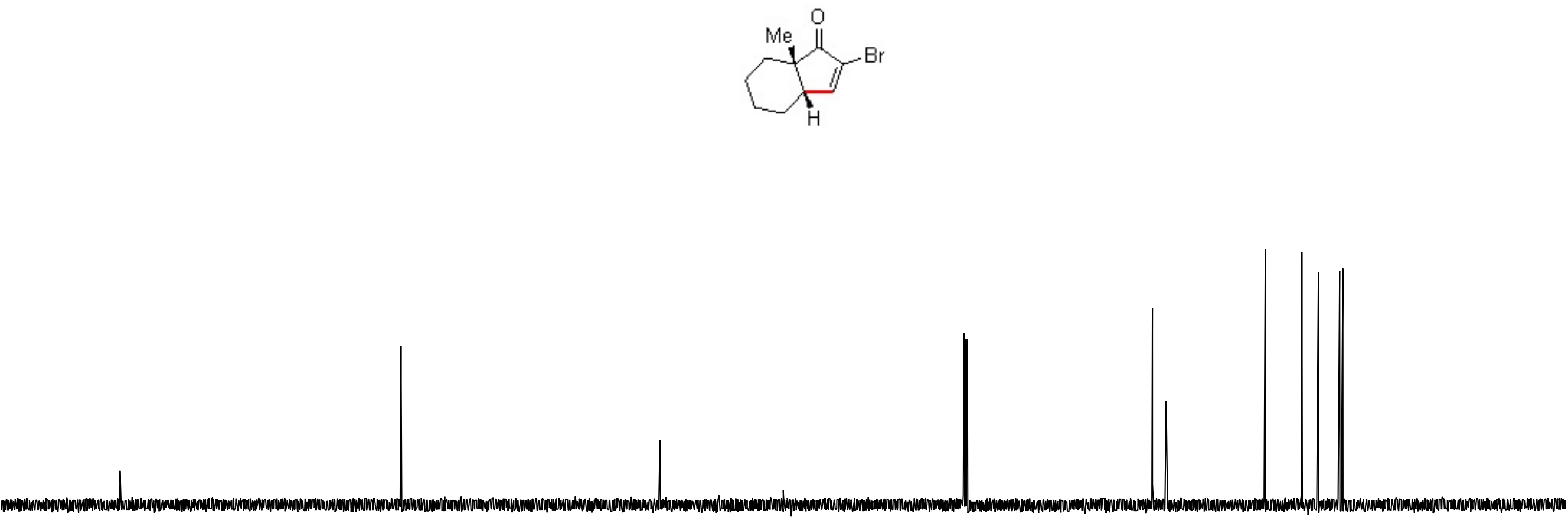

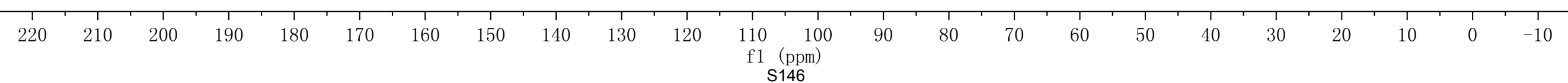




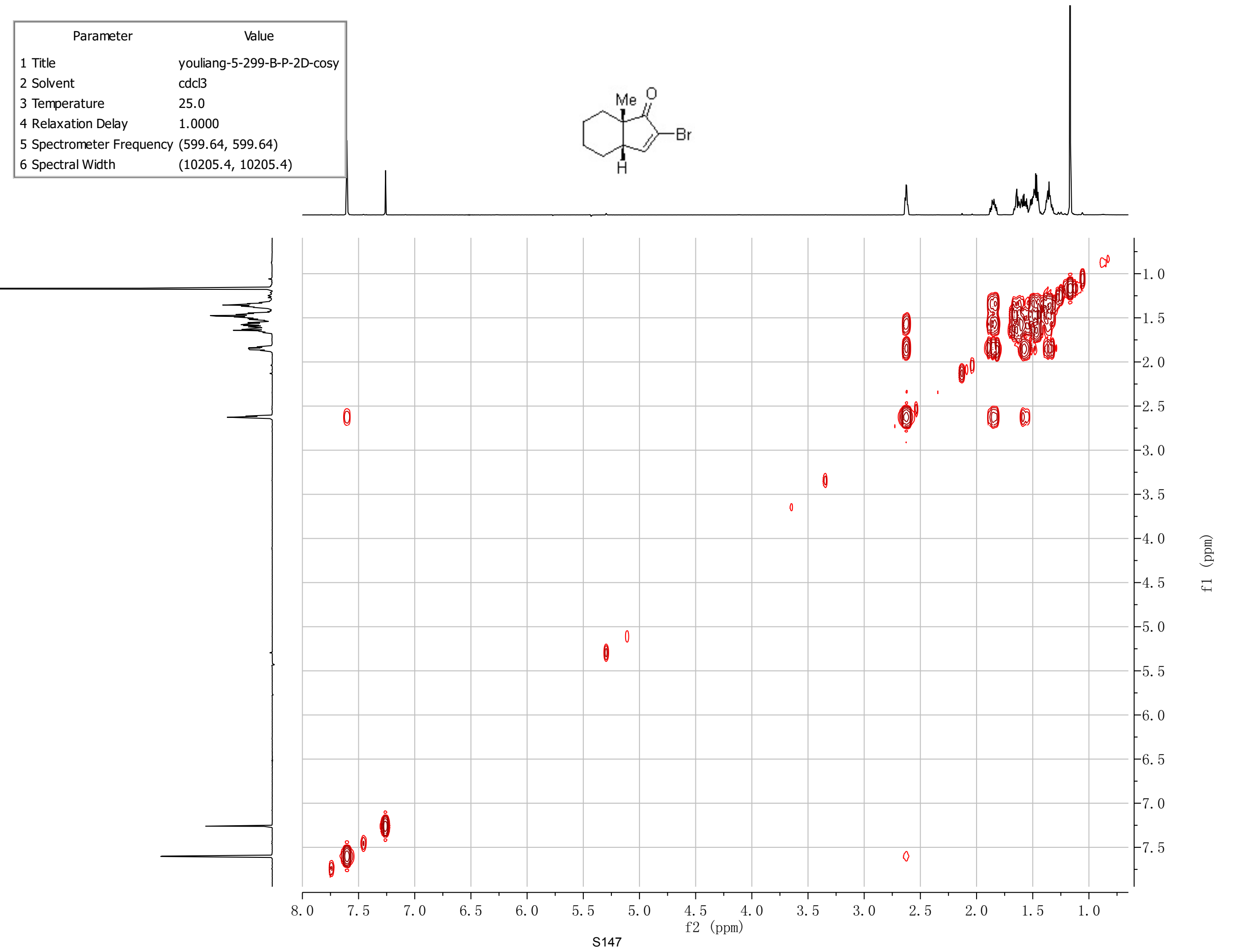




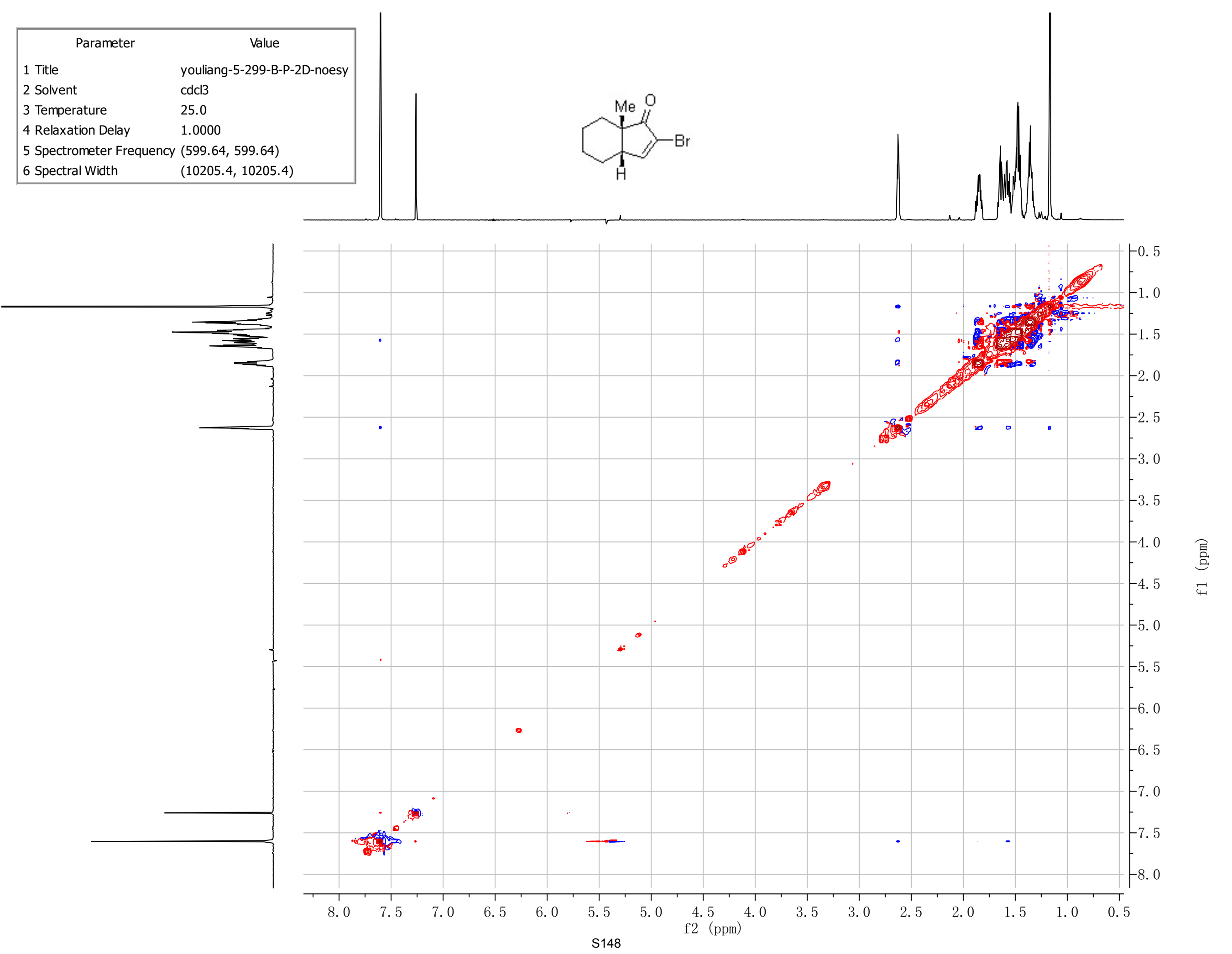




\begin{tabular}{|ll|}
\hline \multicolumn{1}{|c|}{ Parameter } & \multicolumn{1}{c|}{ Value } \\
1 Title & youliang-6-12B-P \\
2 Solvent & CDCl3 \\
3 Spectrometer Frequency & 499.86 \\
4 Nucleus & $1 \mathrm{H}$ \\
\hline
\end{tabular}
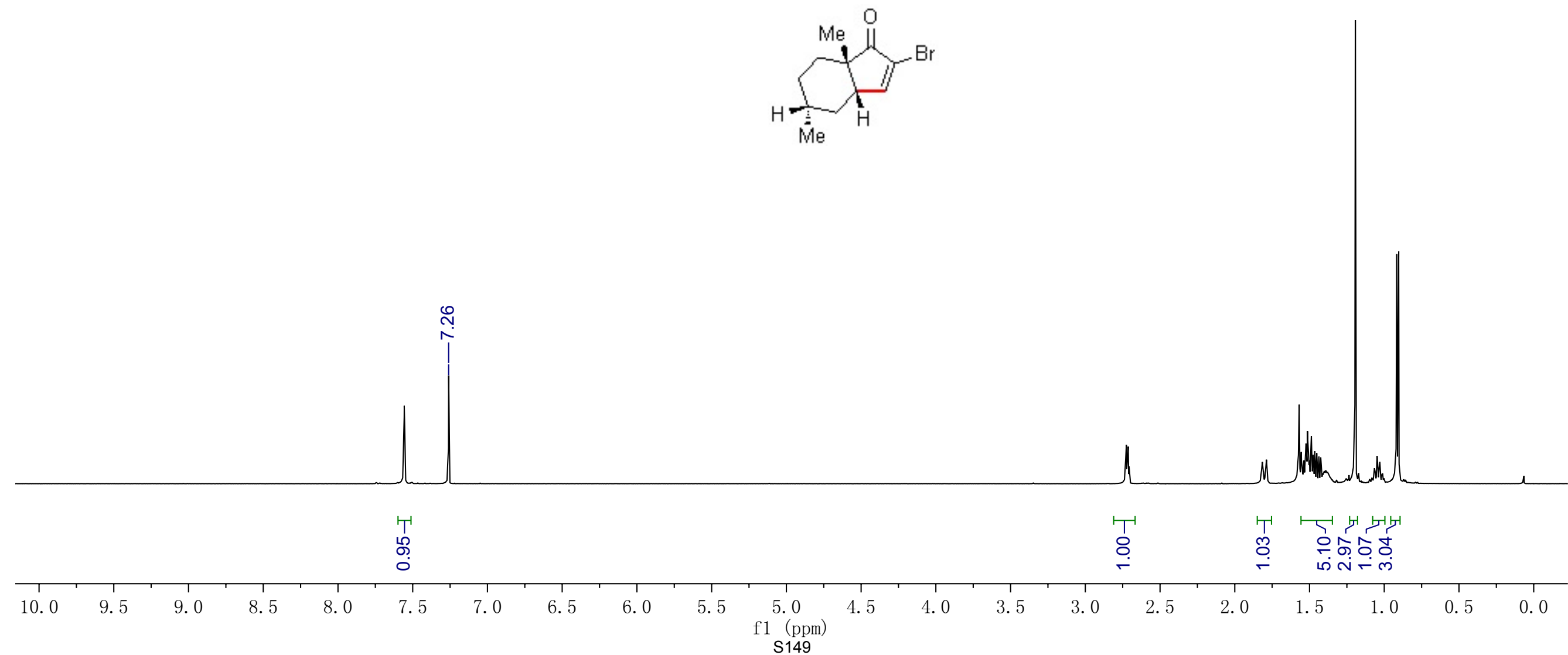

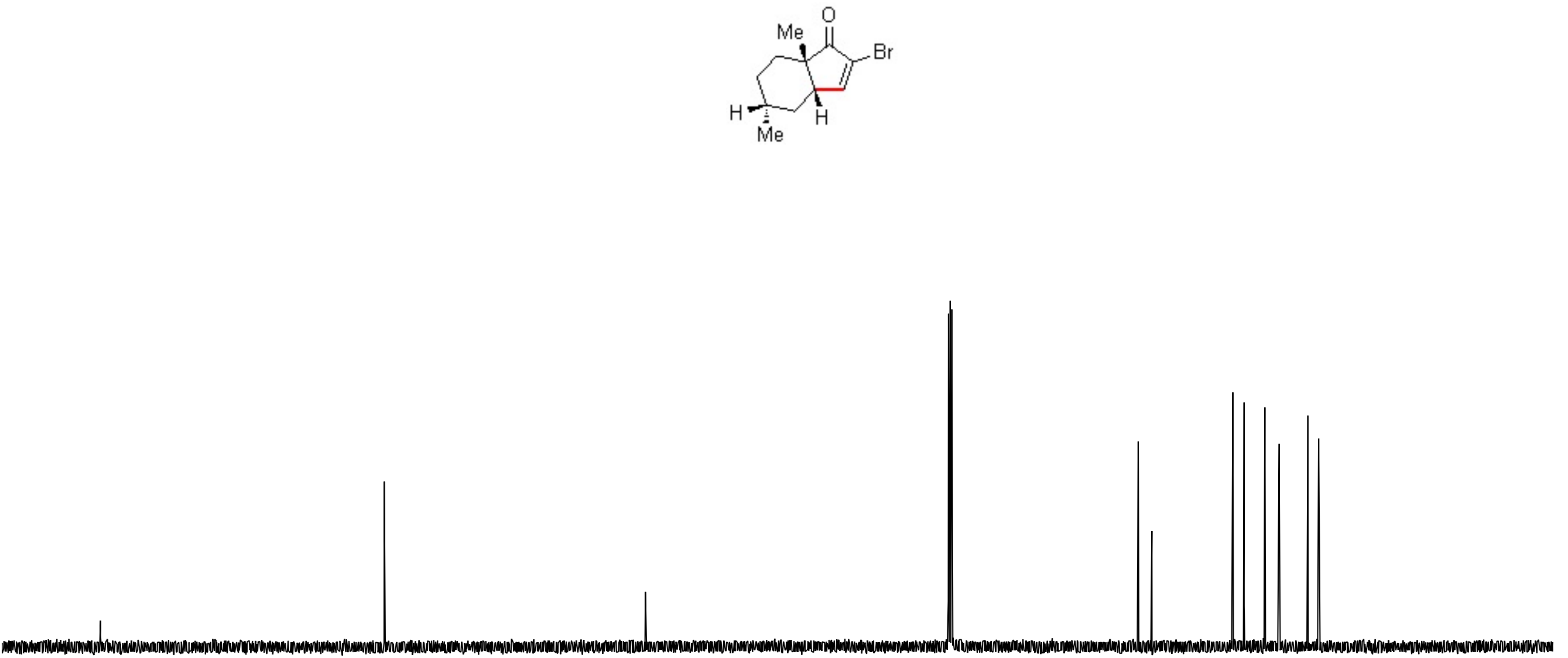

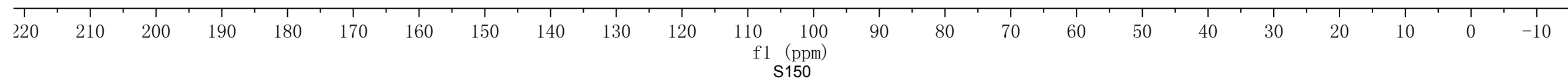




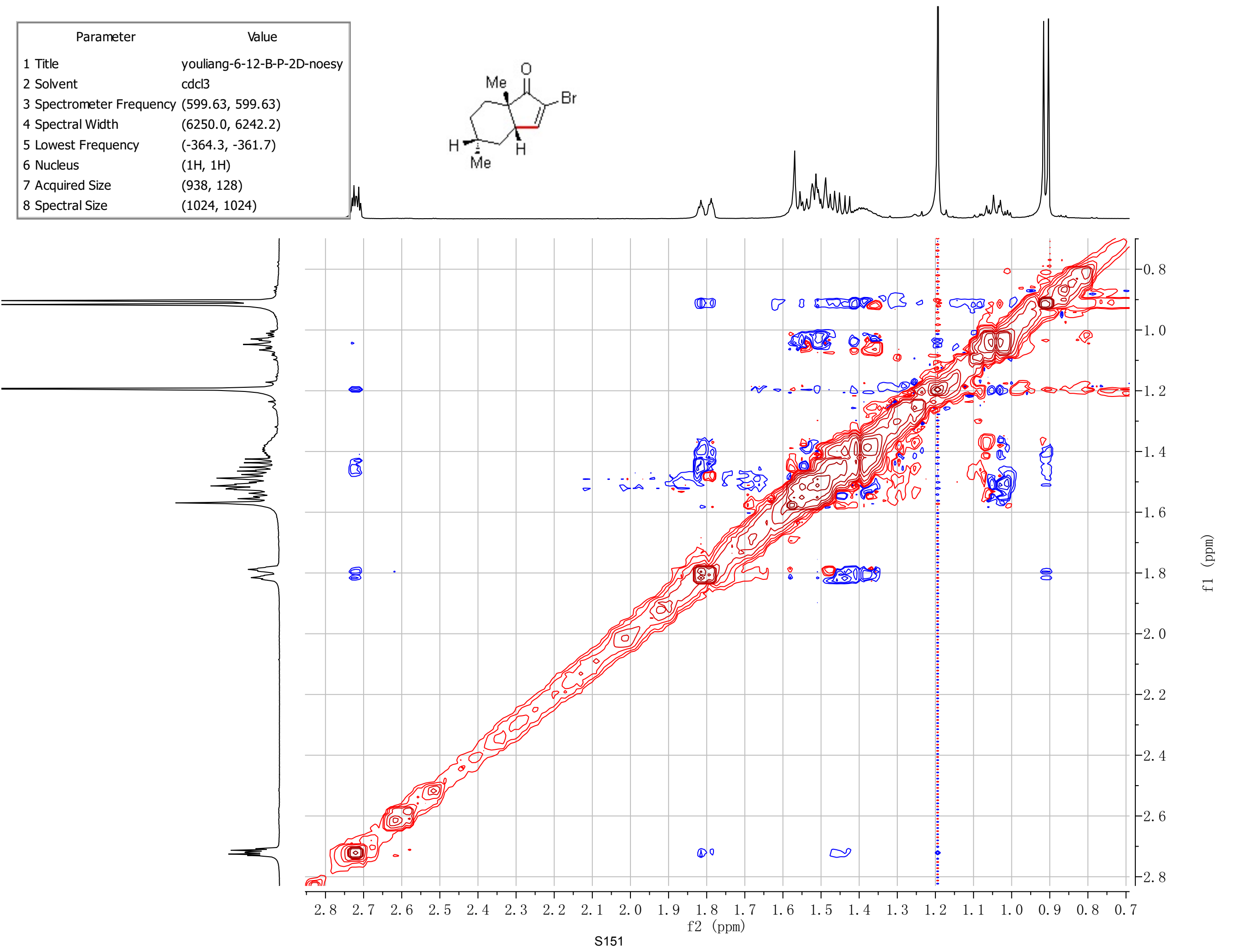




\begin{tabular}{|ll|}
\hline \multicolumn{1}{|c|}{ Parameter } & \multicolumn{1}{c|}{ Value } \\
1 Title & youliang-6-8-B-P \\
2 Solvent & $\mathrm{CDCl3}$ \\
3 Temperature & 25.0 \\
4 Relaxation Delay & 10.0000 \\
5 Spectrometer Frequency & 499.86 \\
\hline
\end{tabular}
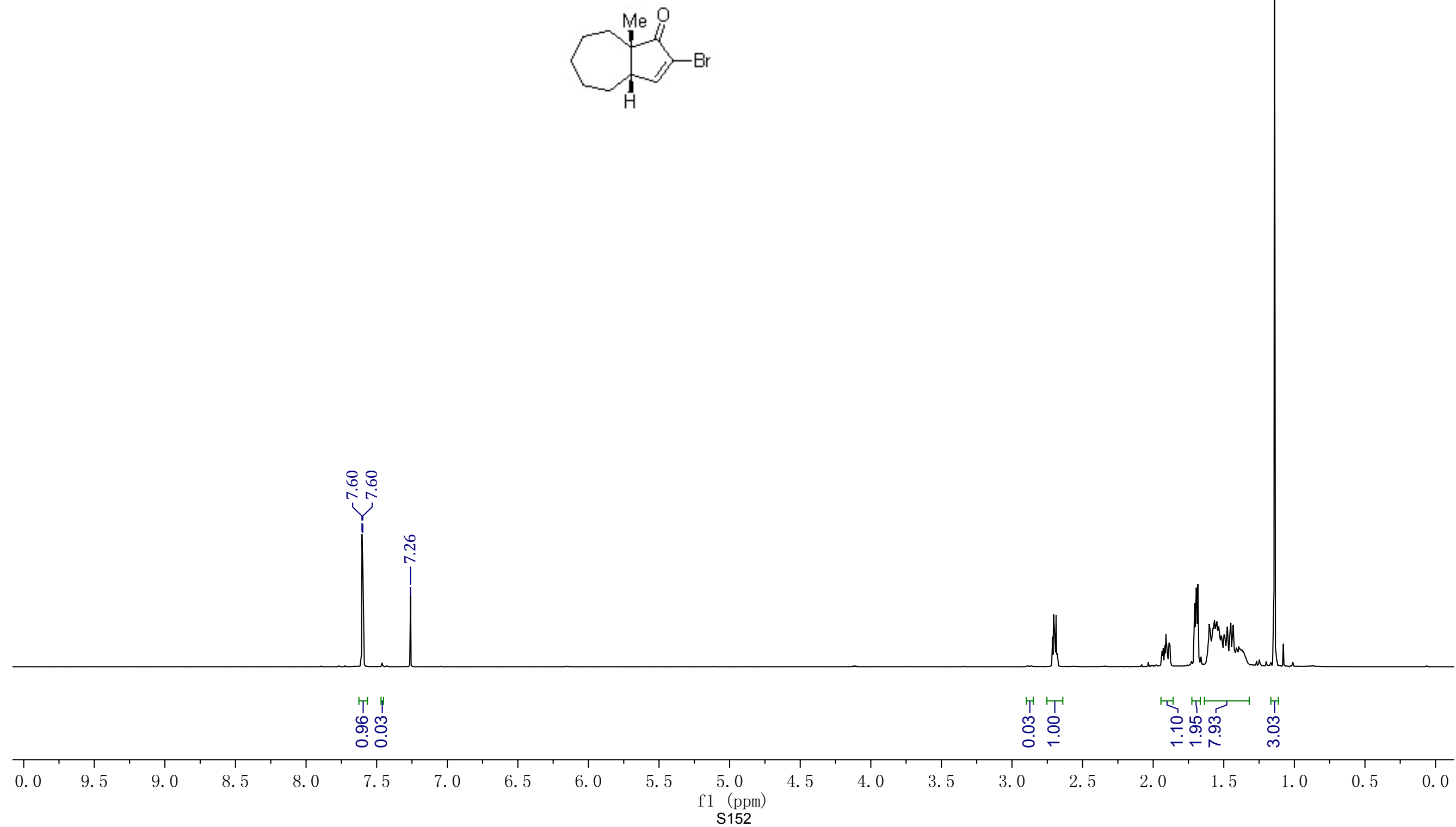


\begin{tabular}{|ll|}
\hline \multicolumn{1}{|c|}{ Parameter } & \multicolumn{1}{c|}{ Value } \\
1 Title & youliang-6-8-B-P-C13f \\
2 Solvent & $\mathrm{CDCl} 3$ \\
3 Temperature & 25.0 \\
4 Relaxation Delay & 1.0000 \\
5 Spectrometer Frequency & 125.70 \\
\hline
\end{tabular}
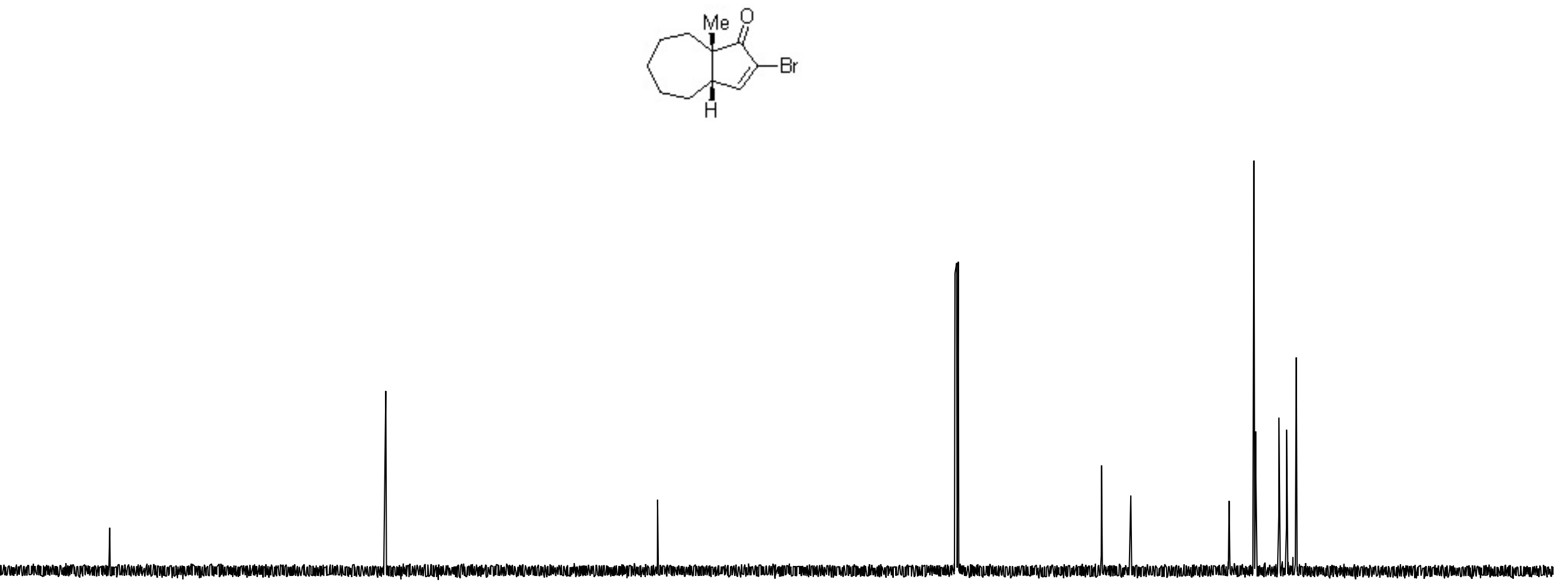

\begin{tabular}{|c|c|c|c|c|c|c|c|c|c|c|c|c|c|c|c|c|c|c|c|c|c|c|c|}
\hline $\begin{array}{c}1 \\
220\end{array}$ & 210 & 200 & $\begin{array}{c}1 \\
190\end{array}$ & $\begin{array}{l}1 \\
180\end{array}$ & 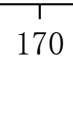 & 160 & $\begin{array}{l}1 \\
150\end{array}$ & 140 & $\begin{array}{l}1 \\
130\end{array}$ & $\begin{array}{l}1 \\
120\end{array}$ & $\begin{array}{r}110 \\
\mathrm{f} 1 \\
\mathrm{~S}\end{array}$ & $\begin{array}{l}1 \\
100 \\
\text { (ppm) } \\
153\end{array}$ & $\begin{array}{l}1 \\
90\end{array}$ & $\begin{array}{l}1 \\
80\end{array}$ & $\begin{array}{l}1 \\
70\end{array}$ & 60 & 50 & 40 & 30 & 20 & 10 & $\begin{array}{l}1 \\
0\end{array}$ & -10 \\
\hline
\end{tabular}




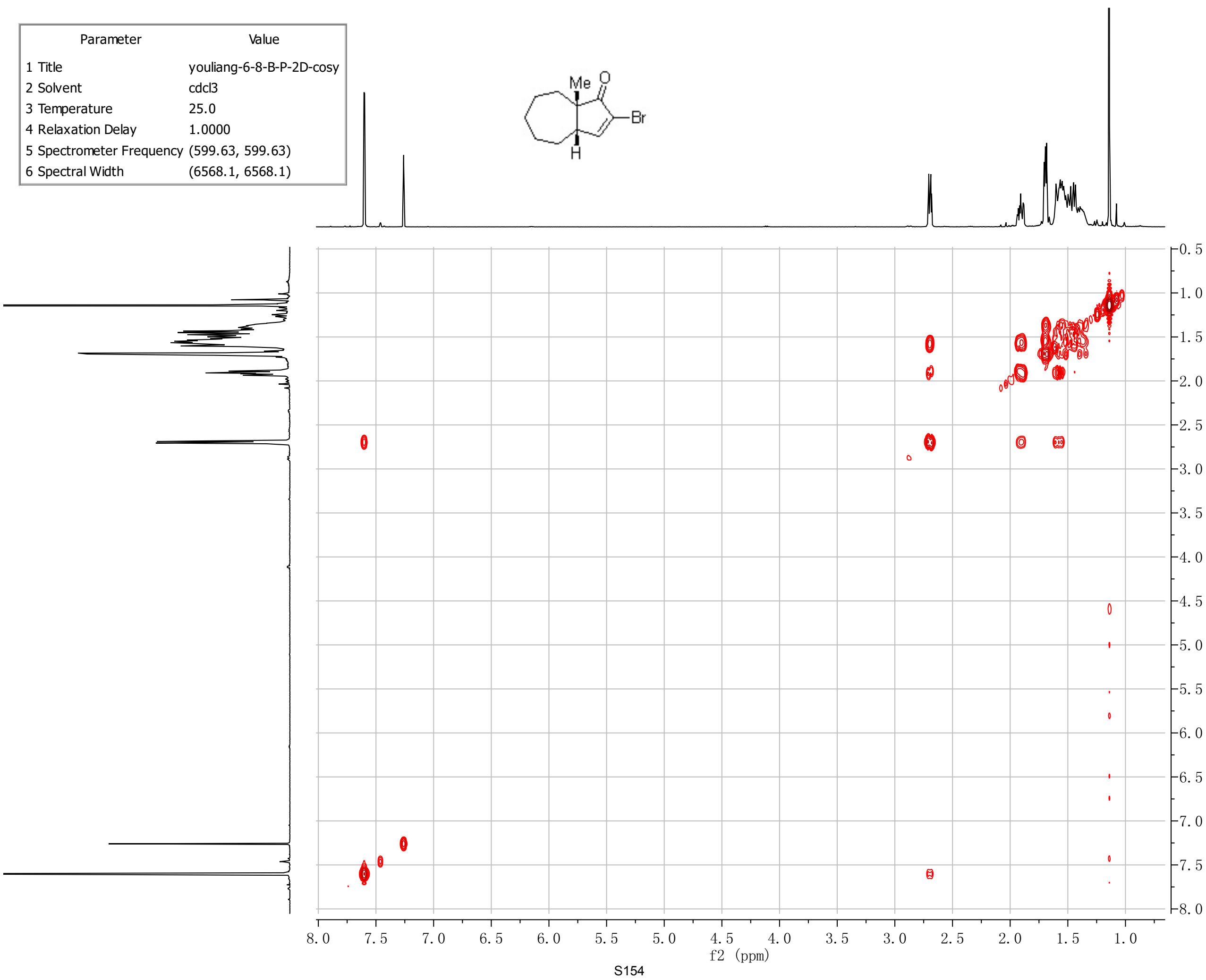




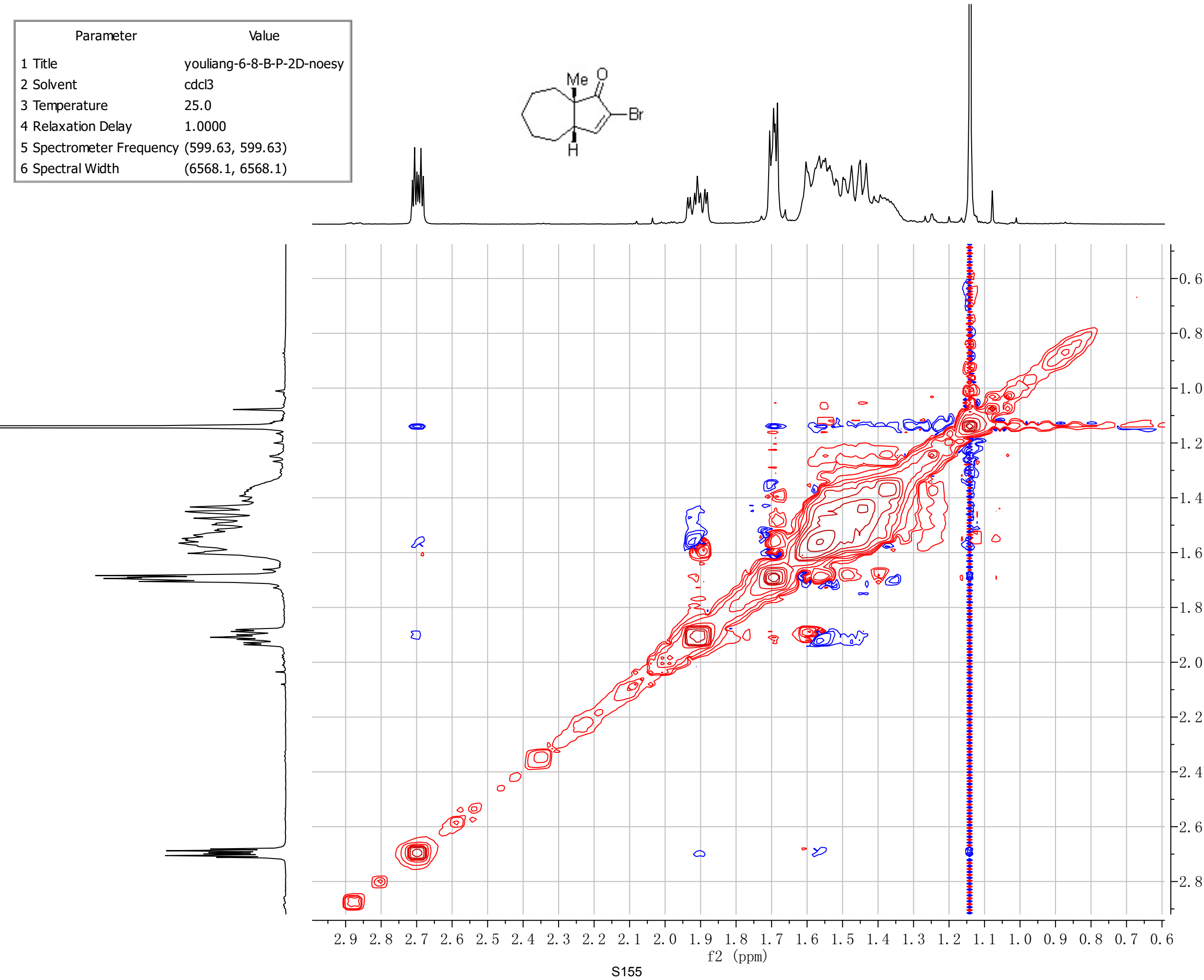




\begin{tabular}{|c|c|}
\hline Parameter & Value \\
\hline 1 Title & youliang-6-58C-P \\
\hline 2 Solvent & $\mathrm{CDCl3}$ \\
\hline 3 Spectrometer Frequenc & 499.86 \\
\hline 4 Nucleus & $1 \mathrm{H}$ \\
\hline
\end{tabular}

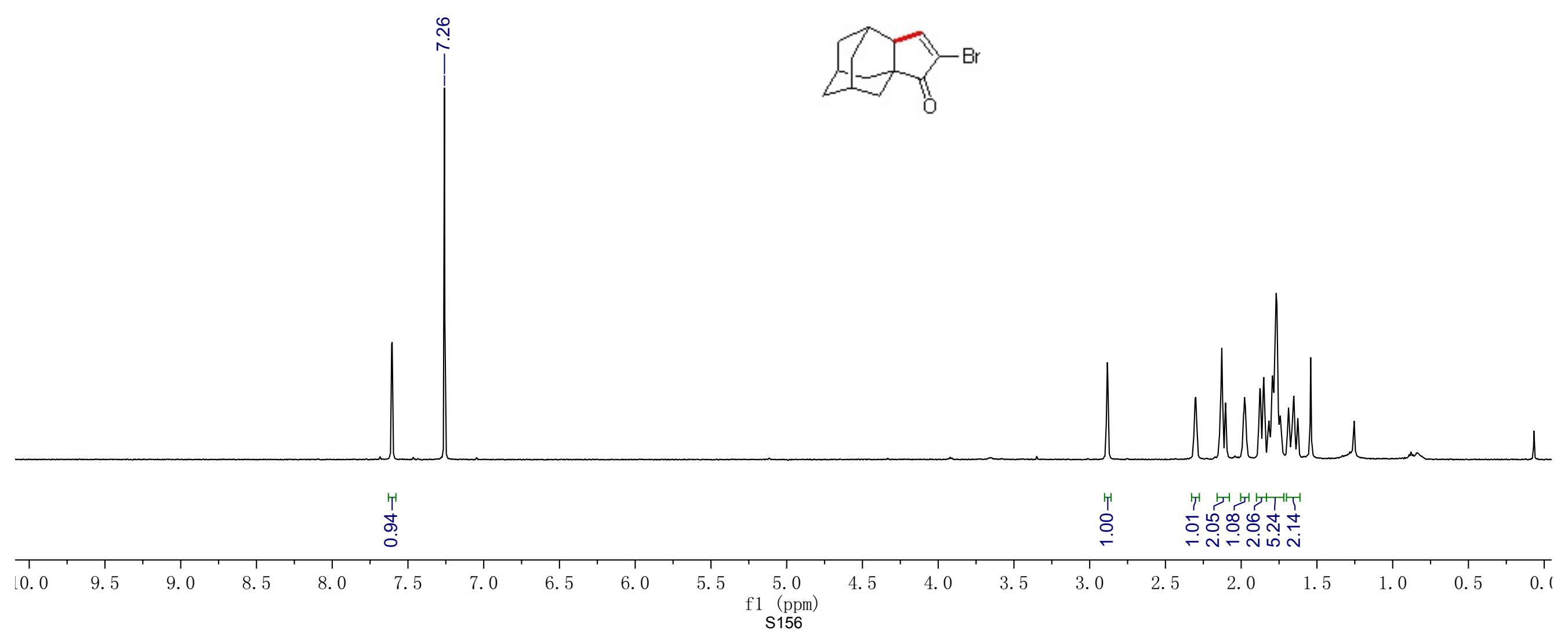




\begin{tabular}{|ll|}
\hline \multicolumn{1}{|c|}{ Parameter } & \multicolumn{1}{c|}{ Value } \\
1 Title & youliang-6-58C-P-C13h \\
2 Solvent & $\mathrm{CDCl3}$ \\
3 Spectrometer Frequency & 125.70 \\
4 Nucleus & $13 \mathrm{C}$ \\
\hline
\end{tabular}

웃윰유ํํㅇ

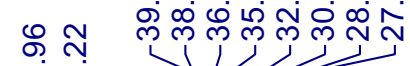

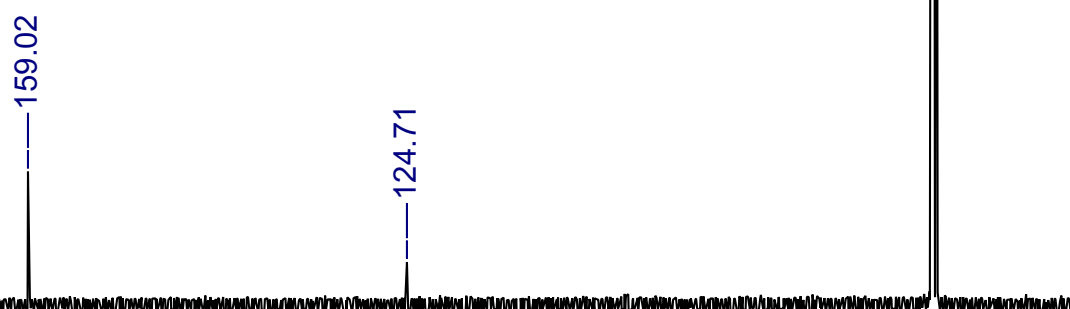

กิ่

นึ)

ڤั่

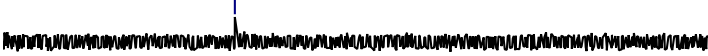

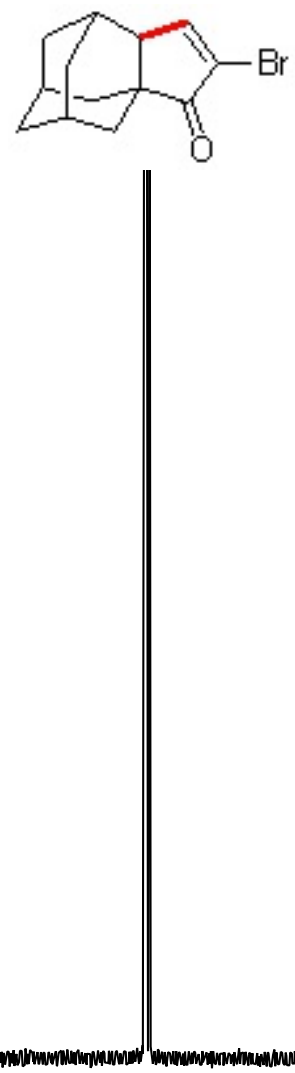

Br
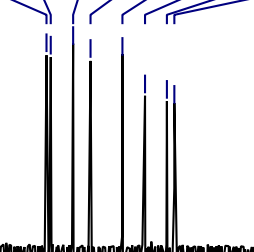

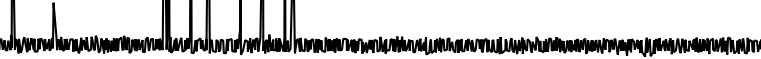

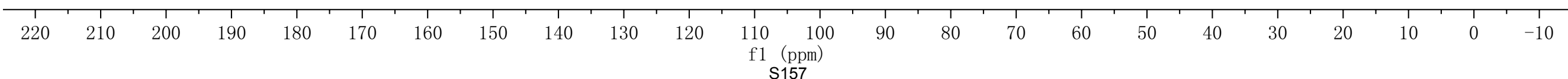




\begin{tabular}{|ll|}
\hline \multicolumn{1}{|c|}{ Parameter } & \multicolumn{1}{c|}{ Value } \\
1 Title & youliang-6-98C-P \\
2 Solvent & $C D C l 3$ \\
3 Spectrometer Frequency & 499.86 \\
4 Nucleus & $1 \mathrm{H}$ \\
\hline
\end{tabular}
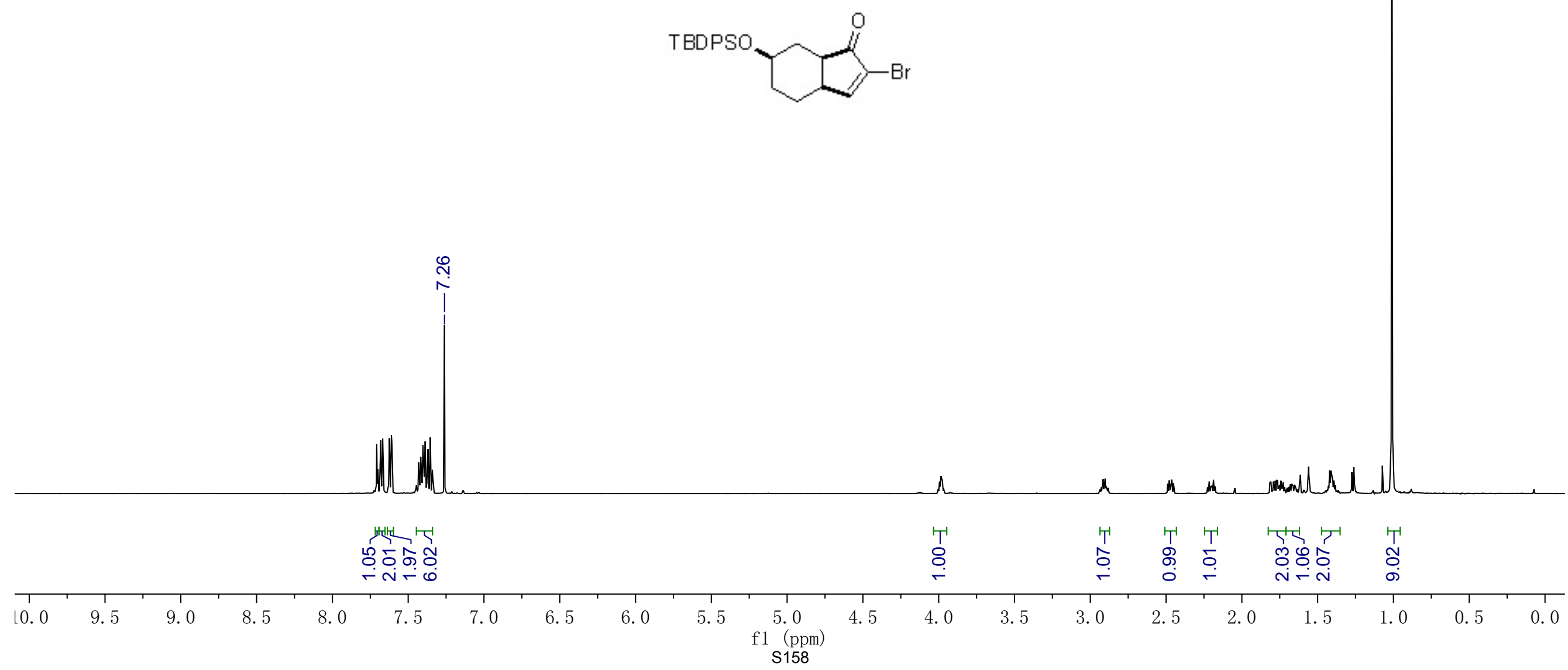


\begin{tabular}{|ll|}
\hline \multicolumn{1}{|c|}{ Parameter } & \multicolumn{1}{c|}{ Value } \\
1 Title & youliang-6-98C-P-C13K \\
2 Solvent & $\mathrm{CDCl} 3$ \\
3 Spectrometer Frequency & 125.70 \\
4 Nucleus & $13 \mathrm{C}$ \\
\hline
\end{tabular}
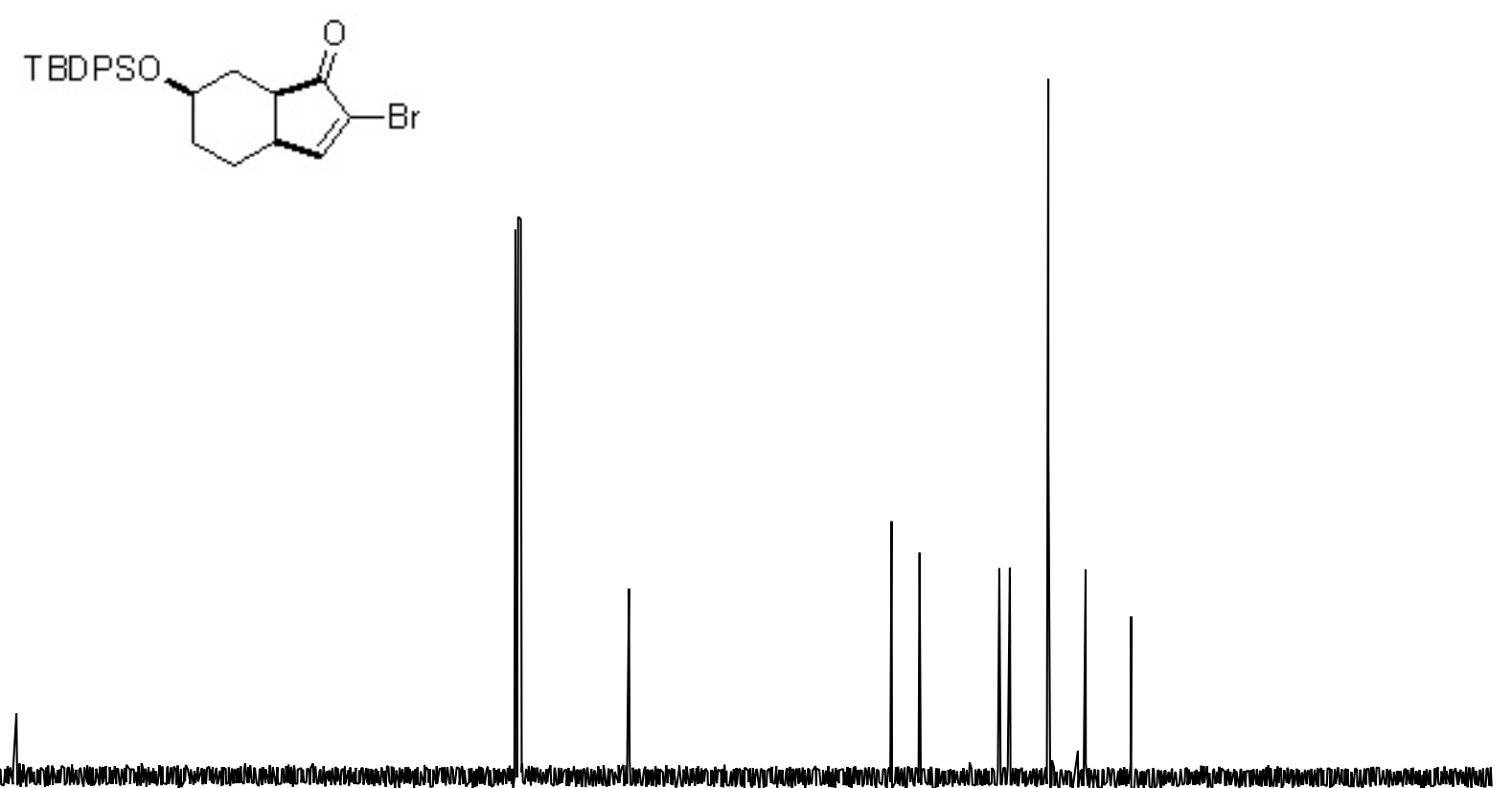

\begin{tabular}{|c|c|c|c|c|c|c|c|c|c|c|c|c|c|c|c|c|c|c|c|c|c|c|c|}
\hline $\begin{array}{c}1 \\
220\end{array}$ & 210 & $\begin{array}{c}1 \\
200\end{array}$ & $\begin{array}{r}1 \\
190\end{array}$ & $\begin{array}{r}1 \\
180\end{array}$ & $\begin{array}{c}1 \\
170\end{array}$ & 160 & $\begin{array}{r}1 \\
150\end{array}$ & 140 & 130 & $\begin{array}{c}1 \\
120\end{array}$ & $\begin{array}{r}110 \\
\mathrm{f} 1 \\
\mathrm{~S}\end{array}$ & $\begin{array}{c}1 \\
100 \\
\text { ppm) } \\
59\end{array}$ & 90 & $\begin{array}{l}1 \\
80\end{array}$ & $\begin{array}{c}1 \\
70\end{array}$ & 60 & $\begin{array}{c}1 \\
50\end{array}$ & $\begin{array}{c}1 \\
40\end{array}$ & $\begin{array}{c}1 \\
30\end{array}$ & $\begin{array}{l}1 \\
20\end{array}$ & 10 & 1 & -10 \\
\hline
\end{tabular}



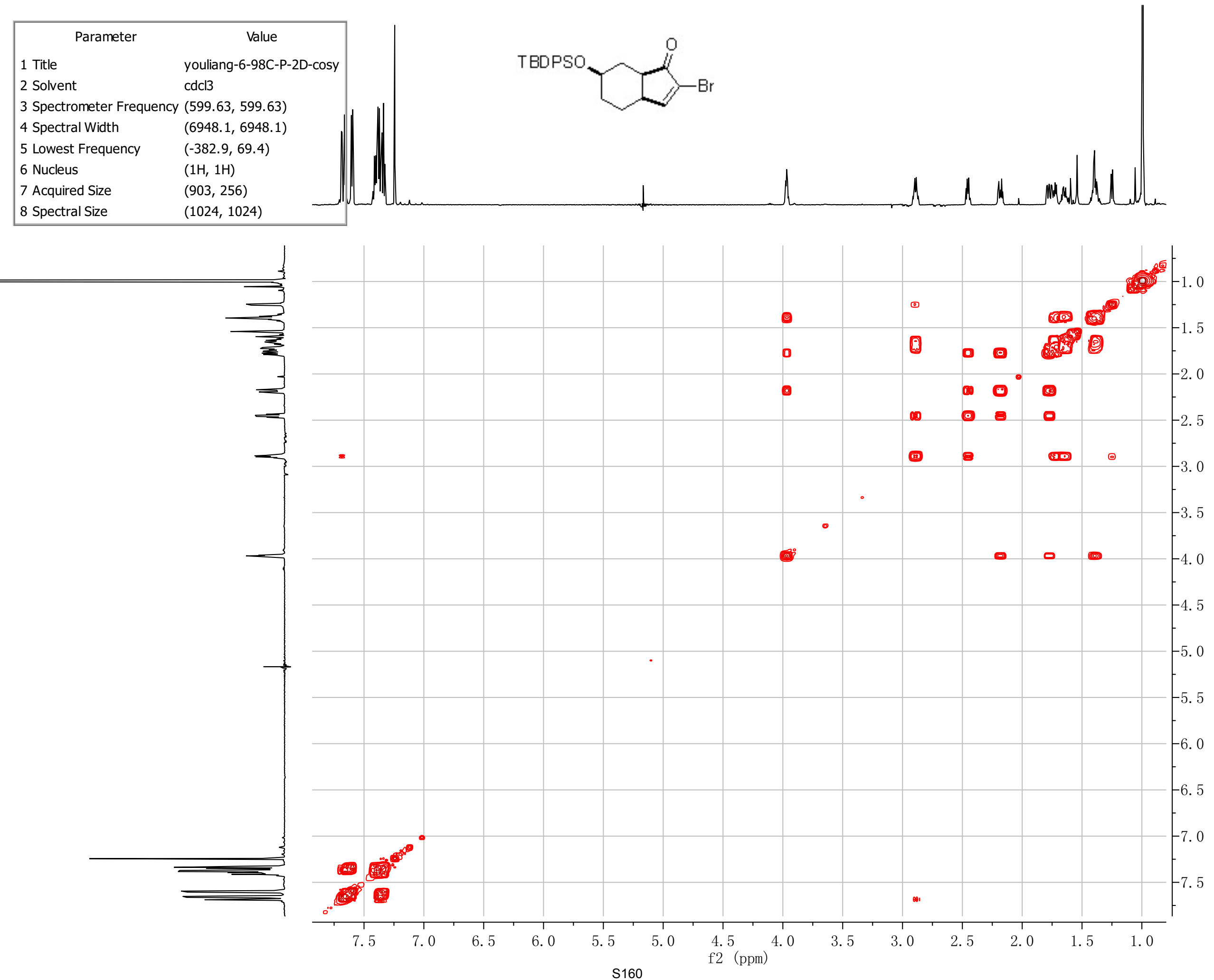


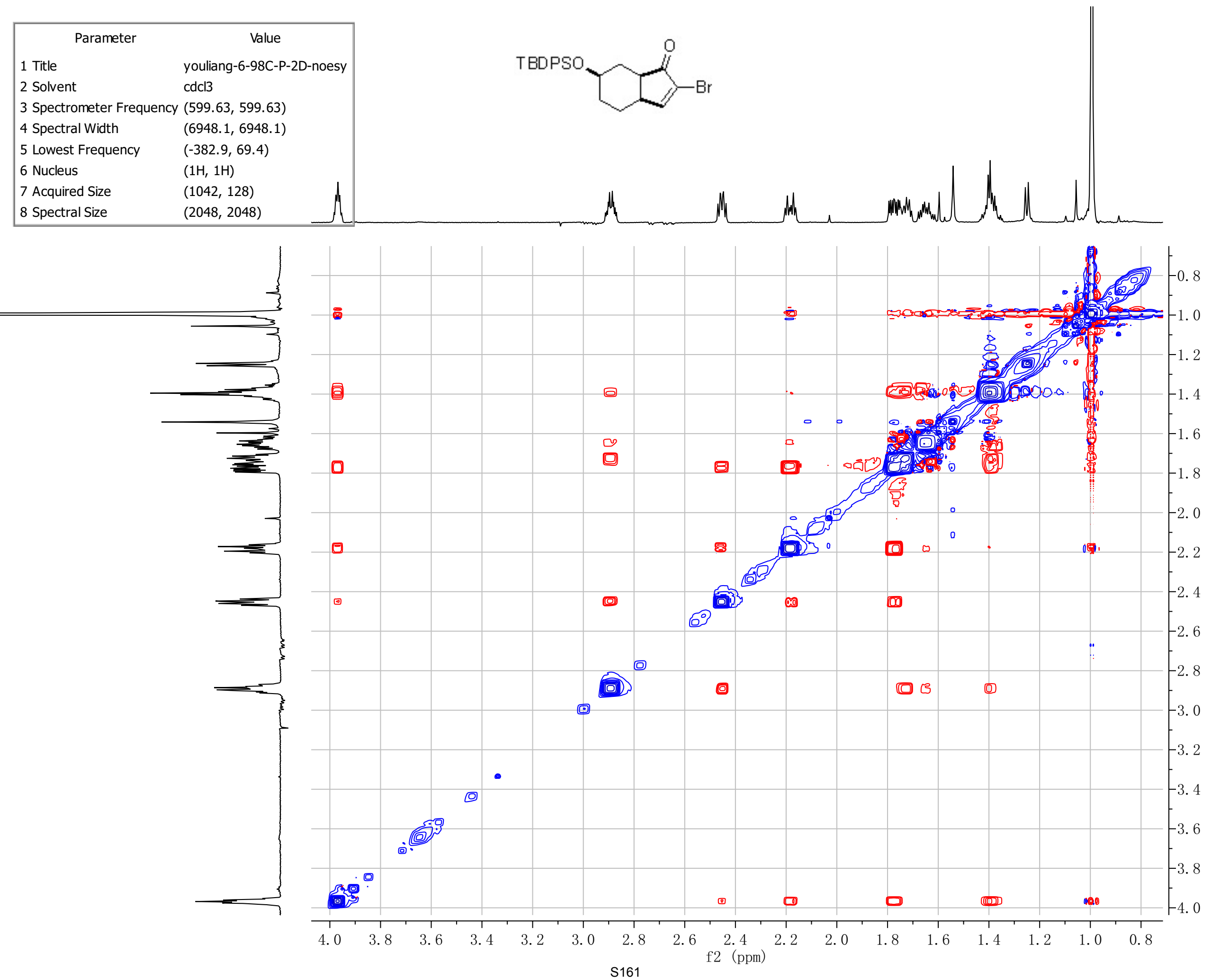




\begin{tabular}{|ll|}
\hline \multicolumn{1}{|c|}{ Parameter } & \multicolumn{1}{c|}{ Value } \\
1 Title & youliang-6-101E-P \\
2 Solvent & cdcl3 \\
3 Spectrometer Frequency & 399.78 \\
4 Nucleus & $1 \mathrm{H}$ \\
\hline
\end{tabular}
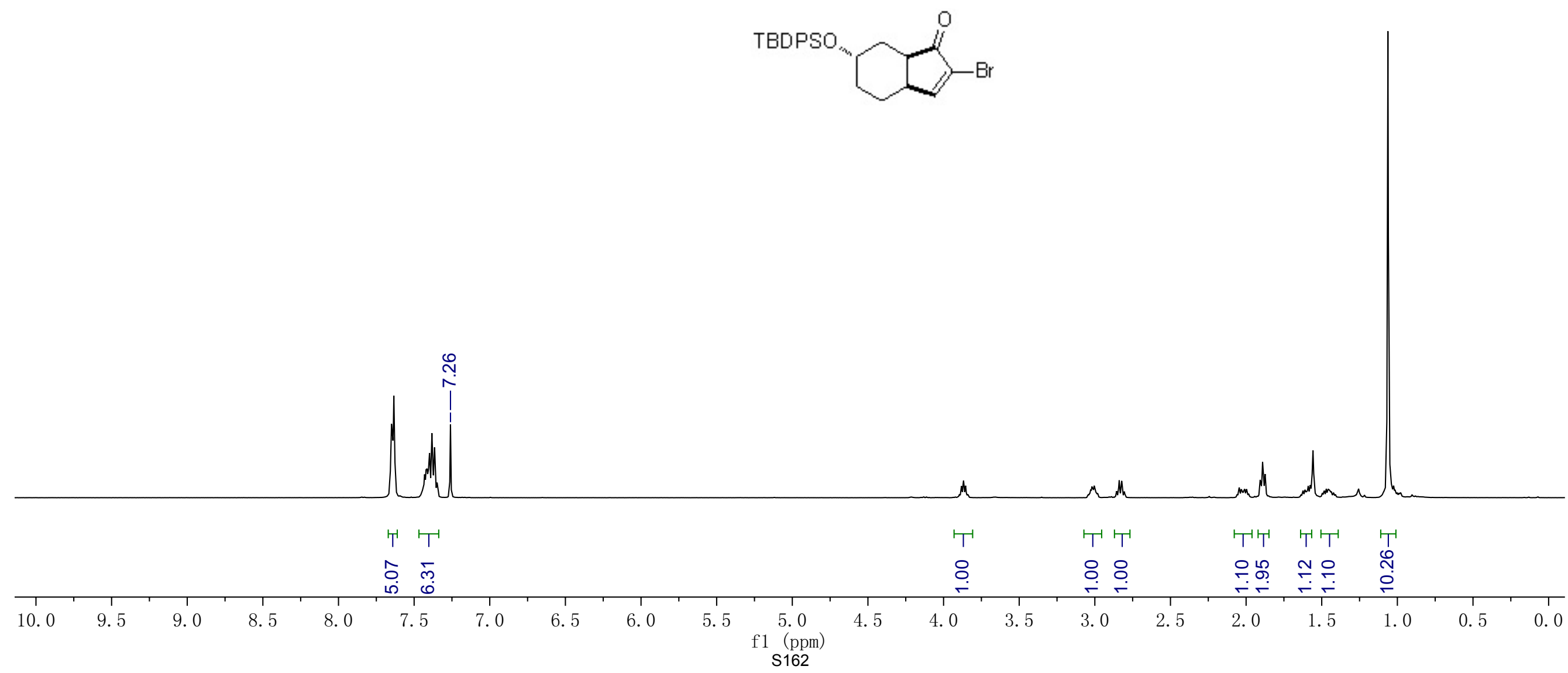


\begin{tabular}{|ll|}
\hline \multicolumn{1}{|c|}{ Parameter } & \multicolumn{1}{c|}{ Value } \\
1 Title & youliang-6-101E-P-C13x \\
2 Solvent & $\mathrm{CDCl} 3$ \\
3 Spectrometer Frequency & 125.70 \\
4 Nucleus & $13 \mathrm{C}$ \\
\hline
\end{tabular}
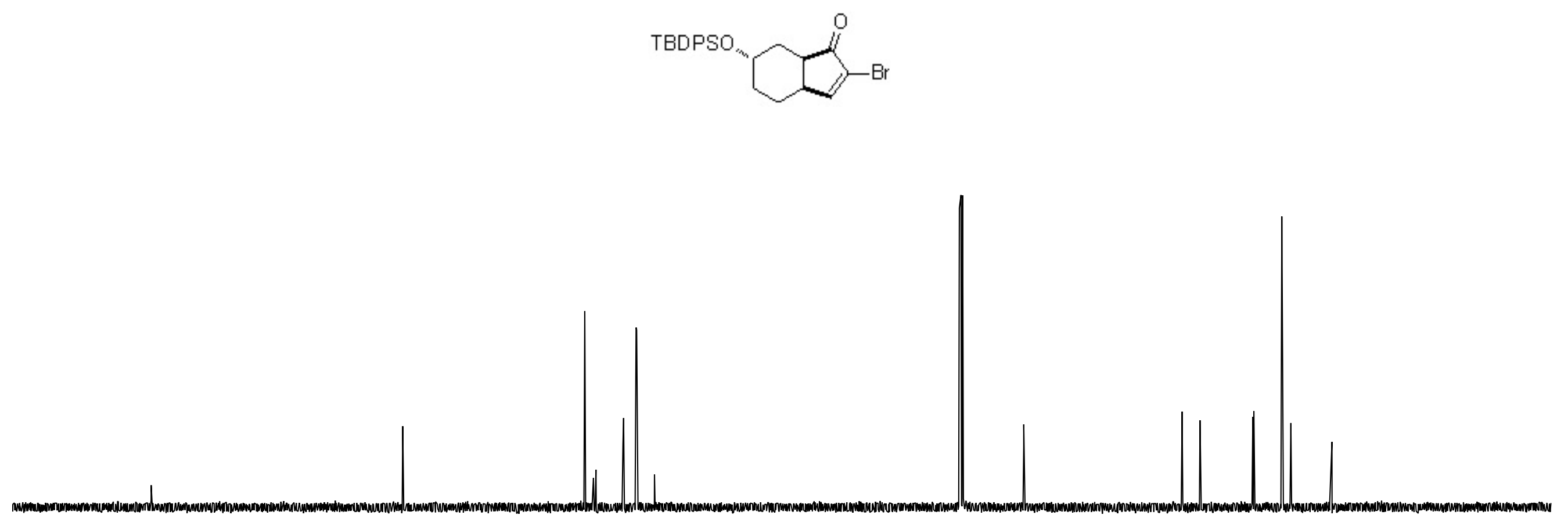

\begin{tabular}{|c|c|c|c|c|c|c|c|c|c|c|c|c|c|c|c|c|c|c|c|c|c|c|c|}
\hline 220 & 210 & $\begin{array}{l}1 \\
200\end{array}$ & $\begin{array}{l}190 \\
190\end{array}$ & $\begin{array}{l}1 \\
180\end{array}$ & 170 & 160 & 11 & 140 & $\begin{array}{l}1 \\
130\end{array}$ & $\begin{array}{l}1 \\
120\end{array}$ & $\begin{array}{r}110 \\
\mathrm{f} 1 \\
\mathrm{~S}\end{array}$ & $\begin{array}{c}100 \\
\text { (ppm) } \\
163\end{array}$ & $\begin{array}{l}1 \\
90\end{array}$ & $\begin{array}{l}1 \\
80\end{array}$ & $\begin{array}{l}1 \\
70\end{array}$ & 60 & 50 & $\begin{array}{l}1 \\
40\end{array}$ & 30 & $\begin{array}{l}1 \\
20\end{array}$ & 10 & $\begin{array}{l}1 \\
0\end{array}$ & $\begin{array}{r}10 \\
-10\end{array}$ \\
\hline
\end{tabular}




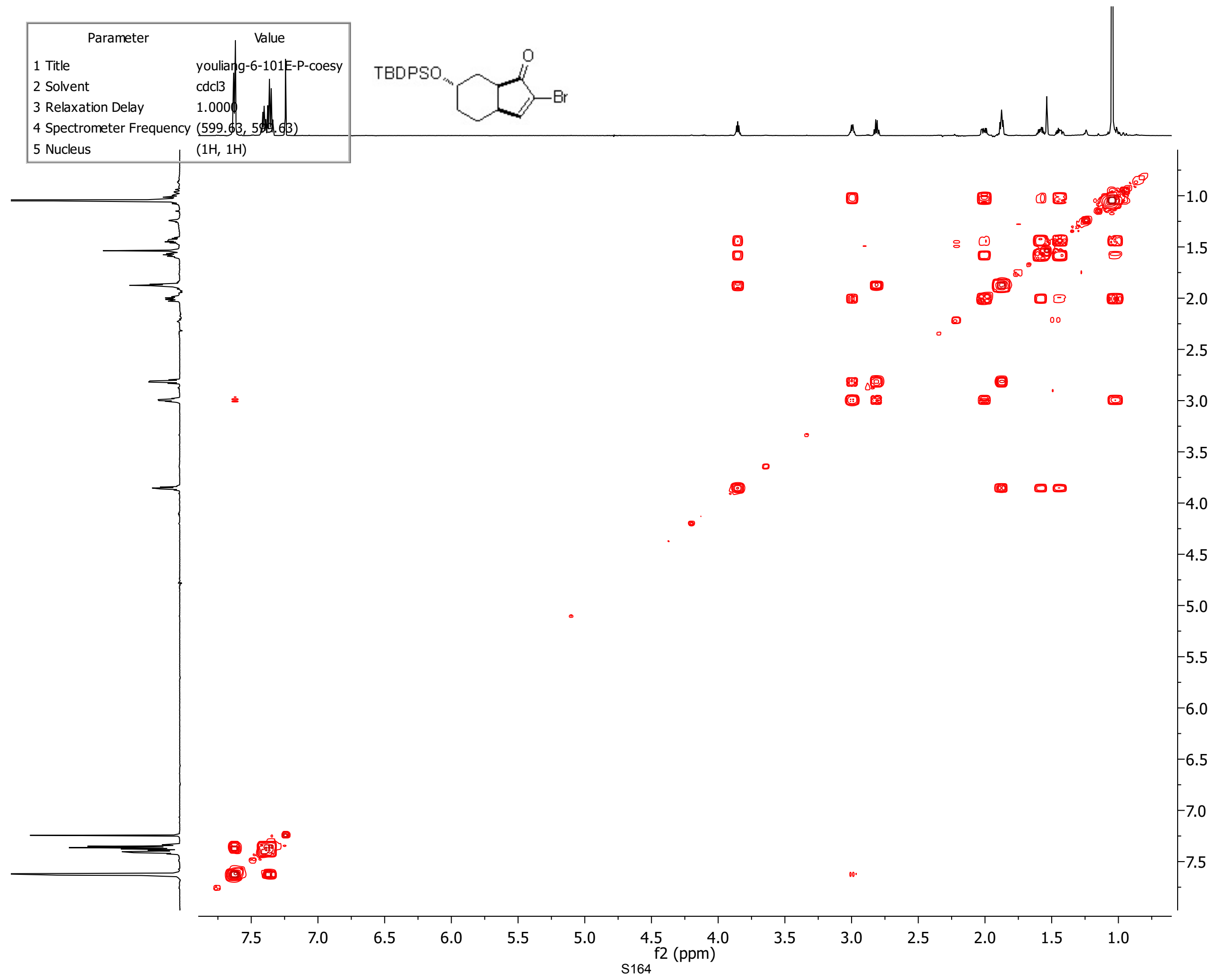




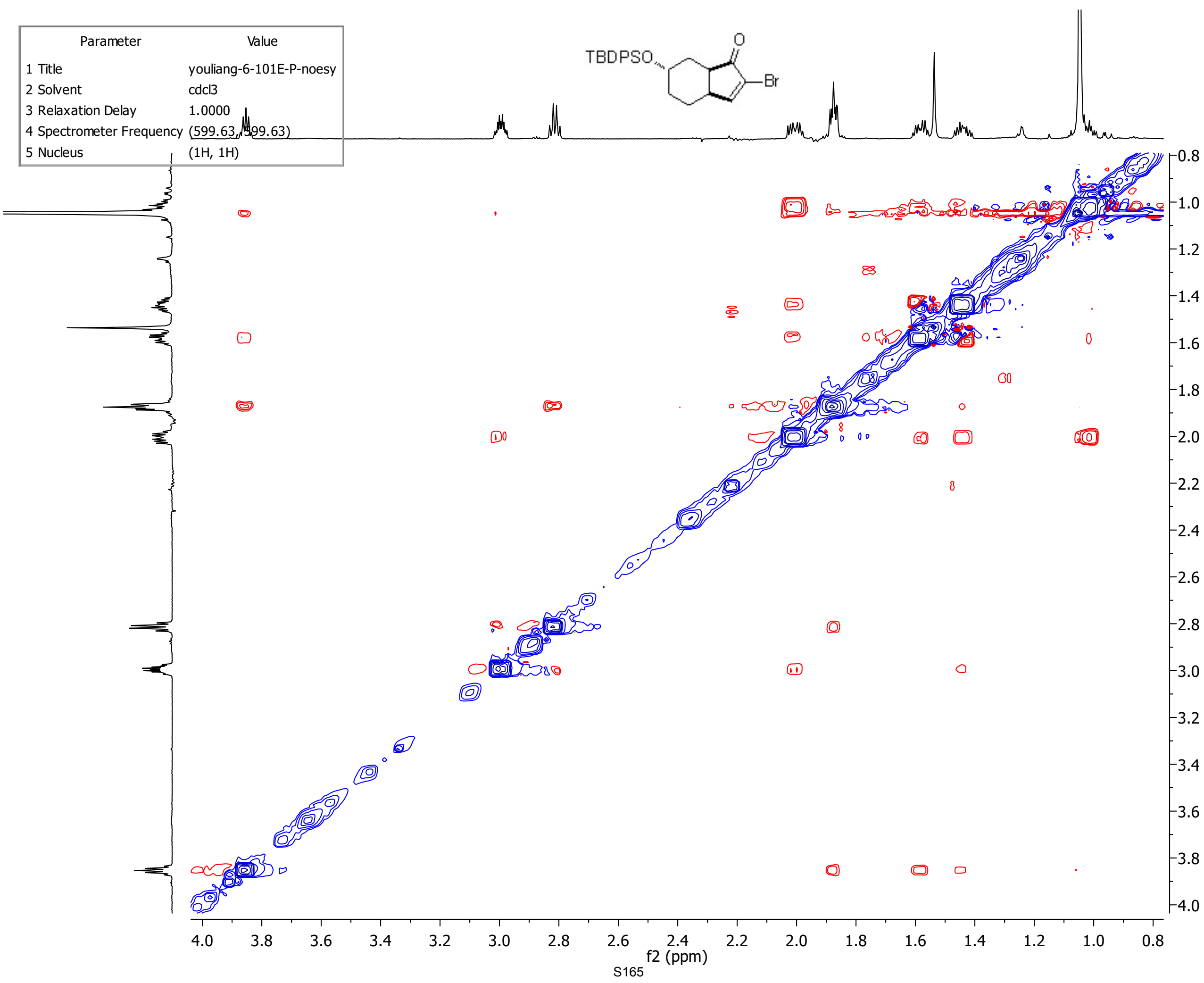

